Modelo de calibração ultraestrutural

\author{
Alina Marcondes Talarico
}



SERVIÇO DE PÓS-GRADUAÇÃO DO ICMC-USP

Data de Depósito:

Assinatura:

\title{
Modelo de calibração ultraestrutural
}

\author{
Alina Marcondes Talarico
}

Orientadora: Profa. Dra. Reiko Aoki

Dissertação apresentada ao Instituto de Ciências Matemáticas e de Computação - ICMC-USP, como parte dos requisitos para obtenção do título de Mestre em Ciências - Ciências de Computação e Matemática Computacional. VERSÃO REVISADA 
Ficha catalográfica elaborada pela Biblioteca Prof. Achille Bassi e Seção Técnica de Informática, ICMC/USP

com os dados fornecidos pelo(a) autor(a)

\begin{tabular}{|c|c|}
\hline \multirow[t]{3}{*}{$\mathrm{T} 137 \mathrm{~m}$} & $\begin{array}{l}\text { Talarico, Alina Marcondes } \\
\text { Modelo de calibração ultraestrutural / Alina } \\
\text { Marcondes Talarico; orientador Reiko Aoki. -- São } \\
\text { Carlos, } 2014 \text {. } \\
\quad 148 \text { p. }\end{array}$ \\
\hline & $\begin{array}{l}\text { Dissertação (Mestrado - Programa de Pós-Graduação } \\
\text { em Ciências de Computação e Matemática } \\
\text { Computacional) -- Instituto de Ciências Matemáticas } \\
\text { e de Computação, Universidade de São Paulo, } 2014 \text {. }\end{array}$ \\
\hline & $\begin{array}{l}\text { 1. Modelo de Calibração. 2. Comparação } \\
\text { interlaboratorial. 3. Distribuição normal } \\
\text { multivariada. 4. Distribuição t multivariada. I. } \\
\text { Aoki, Reiko, orient. II. Título. }\end{array}$ \\
\hline
\end{tabular}




\section{Sumário}

1 Introdução $\quad 8$

1.1 Revisão bibliográfica . . . . . . . . . . . . . . . . . . 12

1.2 Motivação . . . . . . . . . . . . . . . . . . . 14

2 Modelo de Calibração Normal $\quad 18$

2.1 Função escore . . . . . . . . . . . . . . . . . . . . 21

2.2 Matriz de informação observada . . . . . . . . . . . . . . . 22

2.3 Estimação dos parâmetros e testes de hipóteses . . . . . . . . 25

2.3 .1 Algoritmo EM . . . . . . . . . . . . . . 25

2.3 .2 Testes de hipóteses assintóticos . . . . . . . . . . . 29

2.3 .3 Estimação restrita sob $H_{0} \ldots \ldots \ldots \ldots$

3 Modelo de Calibração t de Student 35

3.1 Função escore e matriz de informação observada . . . . . . . . . . . 36

3.2 Estimação dos parâmetros e testes de hipóteses . . . . . . . . . . 38

3.2 .1 Algoritmo EM . . . . . . . . . . . . . . . 39

3.2 .2 Testes de hipóteses assintóticos . . . . . . . . . 40

3.2 .3 Estimação restrita sob $H_{0} \ldots \ldots$. . . . . . . . . 40

4 Aplicação $\quad 45$

4.1 Resultados utilizando a distribuição Normal . . . . . . . . . . . . . 45

4.2 Resultados utilizando a distribuição t de Student . . . . . . . . . . 49

4.2.1 Comparação dos resultados dos testes Normal e t de Student . 55

$\begin{array}{lll}5 & \text { Estudos de Simulação } & 57\end{array}$

5.1 Resultados da simulação para o valor empírico: distribuições Normal e t de Student . . . . . . . . . . . . . . . 59 
5.2 Resultados da simulação para o poder do teste: distribuições Normal e t de Student ....................... 71

6 Conclusão final $\quad 91$

7 Trabalhos Futuros $\quad 92$

8 Referências bibliográficas $\quad 93$

9 Apêndice A $\quad 99$

10 Apêndice B $\quad 103$

11 Apêndice C $\quad 115$

12 Apêndice D $\quad 146$ 
"A vida é construída nos sonhos e concretizada no amor."

Chico Xavier 


\section{Agradecimentos}

A Deus por me dar forças para começar e terminar este trabalho.

Aos meus avós Rosa e Wladimir pelo amor, carinho e confiança que sempre tiveram por mim.

À minha mãe Rosa Maria e ao meu irmão Neto por todo amor e paciência, por terem me direcionado sempre para o caminho certo e, principalmente, por me darem um exemplo a seguir.

À minha cunhada Clarissa pela amizade e pelos momentos de distração e diversão.

Aos amigos de graduação e pós-graduação que tornaram o desenvolvimento deste trabalho menos desgastante.

À minha orientadora Reiko pelos ensinamentos.

Finalmente, à CAPES pelo apoio financeiro.

Pesquisa desenvolvida com o auxílio do LCCA-Laboratório de Computação Científica Avançada da Universidade de São Paulo. 


\section{Resumo}

Os programas de Ensaios de Proficiência (EP) são utilizados pela sociedade para avaliar a competência e a confiabilidade de laboratórios na execução de medições específicas. Atualmente, diversos grupos de EP foram estabelecidos pelo INMETRO, entre estes, o grupo de testes de motores. Cada grupo é formado por diversos laboratórios que medem o mesmo artefato e suas medições são comparadas através de métodos estatísticos. O grupo de motores escolheu um motor gasolina 1.0, gentilmente cedido pela GM Powertrain, como artefato. A potência do artefato foi medida em 10 pontos de rotação por 6 laboratórios. Aqui, motivados por este conjunto de dados, estendemos o modelo de calibração comparativa de Barnett (1969) para avaliar a compatibilidade dos laboratórios considerando a distribuição t de Student e apresentamos os resultados obtidos das aplicações e simulações a este conjunto de dados. 


\section{Abstract}

Proficiency Testing (PT) programs are used by society to assess the competence and the reliability in laboratories execution of specific measurements. Nowadays many PT groups were established by INMETRO, including the motor's test group. Each group is formed by laboratories measuring the same artifact and their measurements are compared through statistic methods. The motor's group chose a gasoline engine 1.0, kindly provided by GM as an artifact. The artifact's power was measured at ten points of rotation by 6 laboratories. Here, motivated by this set data, we extend the Barnet comparative calibration model (1969) to assess the compatibility of the laboratories considering the Student-t distribution and show the results obtained from application and simulation of this set data. 


\section{Introdução}

Um importante mecanismo para as exportações é a confiança que o mercado deposita na rede brasileira de laboratórios. Caso tenhamos problemas nas medições de peças e componentes, não temos como garantir uma boa performance do produto que utiliza tais peças e componentes. O INMETRO (Instituto Nacional de Metrologia, Normalização e Qualidade Industrial) e a sociedade desenvolveram diversos grupos de laboratórios que se reunem periodicamente para avaliar e melhorar seus sistemas de medição. Um sistema de medição corresponde ao conjunto equipamentos, métodos e pessoas, utilizados para obter os resultados da medição. Entre as técnicas aplicadas, está o Ensaio de Proficiência (EP).

O EP corresponde ao uso de comparações interlaboratoriais para determinar a performance de laboratórios para realizar medições específicas ou calibrações de acordo com o Guide 43 (ISO/IEC Guide 43 (1997)). Com esta técnica a consistência e a comparabilidade dos dados do laboratório são monitoradas.

Comparações interlaboratoriais são conduzidas não somente para analisar laboratórios, como também para avaliar métodos e padrões.

Os EP's por meio de comparações interlaboratoriais são utilizados de forma corrente pelos organismos de acreditamento (INMETRO), com o objetivo de avaliar e acompanhar a capacidade de medição dos laboratórios. Eles são reconhecidos internacionalmente como um elemento importante para o estabelecimento da confiança mútua entre os laboratórios de diferentes países e entre organismos nacionais de acreditamento de laboratórios. Um programa de comparação interlaboratorial envolvendo diversos laboratórios requer uma estrutura organizacional adequada e um forte comprometimento dos participantes no cumprimento das condições pré-estabelecidas em documento de orientação.

Os métodos de EP dependem da natureza do item ou material sob teste, do método de ensaio em uso e do número de laboratórios participantes. Existem seis tipos distintos de EP, discutidos no Guide 43: 
Esquemas de comparação de medição: Um único ítem de teste é distribuído sequencialmente entre os laboratórios participantes. Cada laboratório envia o objeto para o próximo laboratório ou ao coordenador para manutenção. Este procedimento é comum em comparações de padrões de calibração. Os resultados das medições, juntamente com as incertezas padrões associadas, devem ser enviadas ao coordenador;

Esquemas de ensaios interlaboratoriais: Vários objetos de teste com características idênticas (supostamente) são produzidos, misturados, embrulhados, e distribuídos para vários participantes. Podem ser distribuídos objetos em duplicata para testar a precisão e exatidão dos laboratórios;

Esquemas de divisão de amostras: Amostras de um produto ou material são divididas em duas ou mais partes com cada laboratório participante testando uma parte de cada amostra;

Esquemas qualitativos: Os objetos de teste têm características conhecidas e categóricas. Os resultados podem ser avaliados independente de outros participantes.

Esquemas de valor conhecido: Os objetos de teste têm características conhecidas e quantitativas. Isso também ocorre quando o objeto de teste é fabricado para produzir uma resposta desejada ou conter uma quantidade conhecida de uma substância. Como no esquema qualitativo, não existe necessidade de se comparar os resultados com outros participantes;

Esquemas de processo parcial: O teste envolve somente partes definidas de um ensaio ou processo de medição. Isso pode ser feito para testar conformidades em certas ações em um laboratório, tais como ajustar uma curva de calibração ou interpretar quantidades radiológicas.

Neste trabalho, consideramos o primeiro tipo de ensaio.

Montanari (2006) considerou o EP de esquemas de comparação de medição, para analisar as medições de um artefato em diferentes temperaturas. Para avaliar o resultado, foi proposto um modelo para explicar os dados e a partir dele foram encontradas as estimativas de máxima verossimilhança (EMV) dos parâmetros do modelo e foi de- 
senvolvida uma estratégia para verificar a compatibilidade dos laboratórios. Neves (2004) propôs um modelo estatístico para dados provenientes de um EP conduzido na área de eletricidade. Os laboratórios participantes mediram um mesmo equipamento padrão devidamente calibrado. Foi feita uma análise para comparar os resultados dos laboratórios em relação a um valor de referência e verificar se o laboratório apresentou algum problema na medição.

Leão, Aoki e Silva (2009) recentemente propuseram um modelo de regressão com erros nas variáveis para testar a competência de laboratórios utilizando a classe de distribuições elípticas para modelar os dados e estabelecer testes estatísticos.

Em adição ao monitoramento da consistência e comparabilidade dos dados obtidos em ensaios, o programa de EP pode contribuir para a melhora dos dados coletados pelos laboratórios, veja Richardson et al. (1996), por exemplo.

A análise dos dados obtidos em um EP segue 3 passos básicos, que são comuns para todos os tipos de programas. Os passos são:

- Determinar o Valor de Referência;

- Fazer a comparação dos resultados;

- Determinar uma estatística de performance.

Os resultados dos participantes devem ser comparados com os valores ou respostas que mais demonstrarem competência com o método.

No nosso caso, no grupo de testes de motores, a GM Powertrain forneceu um motor gasolina 1.0, que circulou entre 6 laboratórios. Cada laboratório mediu a potência do motor em 10 pontos de rotação. O grupo de testes de motores realiza um programa de EP por ano e utiliza os resultados do EP para determinar as ações de melhoria nos laboratórios. Além disso, os resultados do EP são utilizados pelos organismos de acreditação (INMETRO) para avaliar os laboratórios.

Os métodos estatísticos propostos nas normas oficiais (ISO/IEC Guide 43 (1997)) correspondem a aplicações de testes t de Student para cada dois laboratórios e um 
ponto de rotação. No entanto, em muitas situações estamos interessados também em estabelecer comparações múltiplas considerando o grupo de laboratórios participantes.

Neste trabalho estendemos o modelo estrutural definido em Barnett (1969) para o modelo ultra-estrutural com réplicas considerando a distribuição t de Student. Primeiro vamos considerar a distribuição normal e depois estenderemos para a distribuição t de Student.

Sabemos que a inferência baseada na distribuição normal é vulnerável à presença de outliers nos dados que provêm de distribuições com caudas mais pesadas. É um fato reconhecido que os outliers têm uma grande influência sobre os estimadores e testes baseados em máxima verossimilhança. Assim, a distribuição normal pode não ser apropriada em situações em que os dados provêm de distribuições de caudas mais pesadas. Uma possibilidade a fim de resolver este problema é usar distribuições simétricas, com caudas mais pesadas que as da normal, as quais permitem reduzir a influência dos outliers sobre os EMV. A classe de distribuições elípticas tem tido um interesse crescente entre diversos autores. Fang \& Zhang(1990), Berkane et al e Arellano (1994), Leão et al (2009), por exemplo, utilizam essas distribuições. Dentro desta classe, uma das distribuições mais usadas como alternativa à normal é a t de Student.

Vários autores sugerem a distribuição t multivariada como alternativa à normal multivariada por ter as caudas mais pesadas e portanto "acomodar" possíveis outliers presentes nos dados. Além disso, ela produz estimativas mais robustas a observações atípicas. Por exemplo, Rubin (1983) obteve as EMV para os parâmetros da distribuição t multivariada usando o algoritmo EM que é uma ferramenta computacional utilizada para calcular a EMV de forma iterativa, principalmente em problemas envolvendo dados incompletos ; Little (1988) estende os resultados de Rubin (1983) a dados incompletos; Sutradhar e Ali (1986) usam máxima verossimilhança no modelo de regressão multivariados com erros distribuídos t. Portanto estenderemos nosso 
trabalho para a distribuição t de Student. Uma alternativa para modelos com dados correlacionados seria o uso de modelos mistos. Os modelos mistos, por definição, contém efeitos fixos e aleatórios e possibilitam a inclusão de correlação entre indivíduos. São úteis em ambientes onde as medições repetidas são feitas nas mesmas unidades estatísticas. Tais modelos correspondem a uma hierarquia de níveis com ocorrências de medições repetidas e correlacionadas entre os dados da amostra. Na literatura encontramos exemplos de usos de modelos mistos em várias áreas do conhecimento, como, ciências físicas, biológicas e socias. Yang (2008) investigou o problema de previsão de calibração inversa em um cenário de modelo misto. Lesaffre \& Verbeke (1998) usam influência local em modelos mistos para dados longitudinais, Zhu \& Lee (2003) utilizam esquemas de perturbação a modelos lineares mistos generalizados.

Neste trabalho, vamos considerar o modelo de regressão com erros nas variáveis devido às características do nosso conjunto de dados.

\subsection{Revisão bibliográfica}

Em várias áreas do conhecimento, encontramos problemas envolvendo relações entre duas ou mais variáveis. Na química analítica, por exemplo, problemas envolvendo concentrações de substâncias diferentes em uma amostra são muito comuns. Um método geral muito usado para determinar a concentração dessas substâncias na amostra é o de calibração. Uma introdução ao problema de calibração pode ser encontrado em Shukla (1972). Blas (2007) fez uso do modelo de calibração controlada em seu trabalho. Blas, Bolfarine \& Lachos (2011) generalizaram o modelo usado por Blas (2007) assumindo réplicas. A calibração comparativa, que trata da comparação de instrumentos de medição, foi utilizada por Grubbs (1948) em seu trabalho para comparar 3 cronômetros. Barnett (1969) dá um exemplo em que 4 combinações instrumento-operador concebidos para medir a capacidade vital num grupo de pacientes são avaliados. Leurgans (1980) compara 2 métodos para medir a concentração de glicose no sangue. Outros exemplos na área médica podem ser encontrados em 
Kelly (1984,1985). Na área industrial, Jaech (1985), na agricultura, Fuller (1987) e em Psicologia e Educação, Dunn (1992). Rojas (1995) estudou uma sub-classe de modelos com erros nas variáveis, frequentemente usados para comparar instrumentos ou métodos de medição (calibração comparativa). Russo (2006) estudou um problema na área de Odontologia: ajustou um modelo de regressão linear multivariado com erros nas variáveis com intercepto nulo que tratava de medições de placa bacteriana em 3 grupos de voluntários antes e após o uso de 2 bochechos diferentes.

Motivado por um conjunto de dados reais, cedido pela GM Powertrain, nesta dissertação estudaremos um modelo de calibração ultraestrutural, em que o objetivo principal será a comparação dos vícios aditivos e multiplicativos de cada um dos laboratórios com uma medida de referência.

Apesar dos constantes avanços tecnológicos terem tornado cada vez mais precisos os procedimentos de mensuração, não é realista supor que as variáveis sejam medidas sem erros e, o mais comum, é não termos acesso aos seus verdadeiros valores (a presença de erros de mensuração afetará a precisão dos estimadores). Um estudo detalhado sobre esses modelos pode ser encontrado em Kendall \& Stuart (1961), Fuller (1987), Moran (1971) e Cheng\&Van Ness(1999).

Um modelo de regressão linear simples com erros nas váriáveis é definido considerando um conjunto finito de pares $\left(x_{1}, y_{1}\right),\left(x_{2}, y_{2}\right), \ldots,\left(x_{n}, y_{n}\right)$, satisfazendo a relação linear:

$$
y_{i}=\alpha+\beta x_{i}
$$

$i=1, \ldots, n$, em que $\left(x_{i}, y_{i}\right)$ não podem ser observados diretamente, mas com erros através dos pares $\left(X_{i}, Y_{i}\right)$, isto é,

$$
\begin{gathered}
X_{i}=x_{i}+u_{i} \\
Y_{i}=y_{i}+e_{i}
\end{gathered}
$$

Os erros $u_{i}$ e $e_{i}$ são variáveis aleatórias (v.a.) com médias 0 e variâncias finitas $\sigma^{2}$ 
e $\sigma_{e}^{2}$, respectivamente, e independentemente distribuídos para diferentes valores de $i$, $i=1, \ldots, n$.

Assim, podem ser propostos pelo menos 3 modelos diferentes; quando os $x_{i}$ são constantes desconhecidas, o modelo é denominado funcional (Kendall \& Stuart, 1973); quando os $x_{i}$ são v.a. com a mesma distribuição, o modelo é estrutural (Moran, 1971); quando os $x_{i}$ são v.a. independentes com médias diferentes e variância em comum, o modelo é ultraestrutural (Dolby, 1976). Dessa forma, podemos notar que o modelo ultraestrutural generaliza os demais modelos citados. O modelo de regressão linear simples com erros nas variáveis descrito anteriormente, sob distribuição Normal, é não identificável (Kendall \& Stuart (1973)) Para se obter um modelo identificável (Cheng \& Van Ness (1999), Rojas (1995)) algumas suposições adicionais são necessárias. Os procedimentos mais comumente utilizados consistem em supor que:

- As razões das variâncias dos erros $\sigma^{2}$ e $\sigma_{e}^{2}$ são conhecidas ou

- A variância dos erros associados a variável $X_{i}$ ou $Y_{i}$ é conhecida ou

- $\alpha$ é conhecido.

Qualquer uma das suposições acima torna o modelo identificável.

Nesse trabalho, estudaremos o modelo ultraestrutural com réplicas. Um aspecto importante do modelo a ser estudado são as componentes da variância. São utilizadas informações externas em relação ao sistema de medição para estimar estes valores e portanto estes valores são fornecidos pelos laboratórios e supostos conhecidos.

\subsection{Motivação}

Vários laboratórios, buscando a comprovação da competência técnica para realizarem medições, comprometeram-se a participar do programa de comparação interlaborial por ensaios de proficiência. Tais ensaios são utilizados pela sociedade para avaliar a competência e a confiabilidade dos laboratórios na execução de medições 
específicas. Diversos grupos de EP foram estabelicidos pelo INMETRO, e entre eles está o grupo de testes de motores. O grupo de motores escolheu um motor gasolina 1.0, gentilmente cedido pela GM Powertrain, como artefato. No nosso caso, os laboratórios participantes (Marelli, Delphi, Mahle, KSPG, Bosch, Maua) realizaram várias medições de potência em 10 pontos de rotação diferentes: 1500, 2000, 2500, 3000, $3500,4000,4500,5000,5500$ e, 6000. O número de réplicas pode ser diferente para diferentes laboratórios. Com essas medições há um monitoramento de consistência e comparabilidade dos dados dos laboratórios.

Seja $y_{i j k}$ a $k$-ésima medição do verdadeiro valor da potência do motor no $j$-ésimo ponto de rotação medido pelo $i$-ésimo laboratório e $x_{j}$ o verdadeiro valor não observável da potência do motor no $j$-ésimo ponto de rotação, $i=1, \cdots, p, j=1, \cdots, m$, $k=1, \cdots, n_{i}$. Para o nosso conjunto de dados, assumindo que $y_{i j k}$ satisfaz a relação linear ultraestrutural com o verdadeiro valor (não observável) $x_{j}$ e denotando por $Y_{i j k}$ o valor observado (sujeito a erro de medição) da $k$-ésima medição da potência do motor no $j$-ésimo ponto de rotação obtido pelo $i$-ésimo laboratório, o modelo proposto pode ser representado como

$$
\begin{gathered}
y_{i j k}=\alpha_{i}+\beta_{i} x_{j}, \\
Y_{i j k}=y_{i j k}+e_{i j k},
\end{gathered}
$$

$i=1, \cdots, p, j=1, \cdots, m$ e $k=1, \cdots, n_{i}$ em que $E\left(e_{i j k}\right)=0, V\left(e_{i j k}\right)=\sigma_{i j}^{2}$, $E\left(x_{j}\right)=\mu_{x_{j}}$ e $V\left(x_{j}\right)=\sigma_{x_{j}}^{2}, x_{j}$ independente de $e_{i j k}, i=1, \cdots, p, j=1, \cdots, m$ e $k=1, \cdots, n_{i}$.

O objetivo desta análise é a comparação dos vícios aditivos $\left(\alpha_{i}\right)$ e dos vícios multiplicativos $\left(\beta_{i}\right)$ de cada um dos laboratórios $i$ com a medida de referência $(\alpha=0$ e $\beta=1$ ), ou seja, medidas obtidas por um laboratório acreditado ao INMETRO. Barnett (1969) assume um instrumento de referência para seu modelo que mede $x$ sem vícios. Sem perda de generalidade vamos considerar que o primeiro laboratório 
é o laboratório de referência. Assim, temos que:

$$
Y_{1 j k}=x_{j}+e_{1 j k} \quad \text { e } \quad Y_{i j k}=\alpha_{i}+\beta_{i} x_{j}+e_{i j k}
$$

$i=2, \cdots, p, j=1, \cdots, m$, e $k=1, \cdots, n_{i}$.

Para comparar as medidas dos $p$ laboratórios, nós podemos testar as seguintes hipóteses:

1) $H_{0}: \alpha_{2}=\cdots=\alpha_{p}=0$ e $\beta_{2}=\cdots=\beta_{p}=1$,

2) $H_{0}: \beta_{2}=\cdots=\beta_{p}=1$,

3) $H_{0}: \alpha_{2}=\cdots=\alpha_{p}=0$,

4) $H_{0}: \alpha_{i}=0$ e $\beta_{i}=1, i=2, \cdots, p$,

5) $H_{0}: \alpha_{i}=0, i=2, \cdots, p$,

6) $H_{0}: \beta_{i}=1, i=2, \cdots, p$.

Sob a primeira hipótese, todos os laboratórios estão medindo sem vícios aditivos e multiplicativos e sob a segunda (terceira) hipótese os laboratórios estão medindo sem vícios multiplicativos (aditivos). As hipóteses 4, 5 e 6 referem-se a testes individuais de cada laboratório em relação aos seus vícios. Para testarmos estas hipóteses vamos considerar os testes assintóticos de Wald e razão de verossimilhança.

No Capítulo 2, vamos descrever o modelo normal e desenvolver o algoritmo EM para a obtenção das estimativas de máxima verossimilhança. Para tanto obteremos a função escore e a matriz de Fisher observada. Calcularemos a estimação restrita a Ho, e obteremos as estatísticas do teste para testarmos as hipóteses de interesse descritas acima.

No Capítulo 3, faremos a extensão para a distribuição t de Student e desenvolveremos o algoritmo EM para a obtenção das estimativas de máxima verossimilhança e também obteremos a função escore e a matriz de Fisher observada. Calcularemos a estimação restrita a Ho e obteremos as estatísticas do teste para testarmos as hipóteses de interesse.

No Capítulo 4, vamos aplicar os resultados obtidos aos dados reais descritos na 
Introdução. No Capítulo 5, faremos um estudo de simulação e finalmente no Capítulo 6, vamos descrever a continuação do trabalho. 


\section{Modelo de Calibração Normal}

Considerando o modelo definido em $(1)$ e $(2)$, onde $e_{i j k} \stackrel{i n d}{\sim} N\left(0, \sigma_{i j}^{2}\right), x_{j} \stackrel{i n d}{\sim}$ $N\left(\mu_{x_{j}}, \sigma_{x_{j}}^{2}\right), x_{j}$ independente de $e_{i j k}, i=1, \cdots, p, j=1, \cdots, m$ e $k=1, \cdots, n_{i}$, temos:

$$
Y_{1 j k} \sim N\left(\mu_{x_{j}}, \sigma_{x_{j}}^{2}+\sigma_{1_{j}}^{2}\right)
$$

$\mathrm{e}$

$$
Y_{i j k} \sim N\left(\alpha_{i}+\beta_{i} \mu_{x_{j}}, \beta_{i}^{2} \sigma_{x_{j}}^{2}+\sigma_{i_{j}}^{2}\right)
$$

para $i=2, \ldots, p, j=1, \ldots, m$ e $k=1, \ldots, n_{i}$.

Desta forma, a covariância entre as observações tomadas no mesmo ponto de rotação pelo laboratório de referência ( $i$-ésimo laboratório) é dada por $\sigma_{x_{j}}^{2}\left(\beta_{i}^{2} \sigma_{x_{j}}^{2}\right)$ e a covariância entre as observações do laboratório de referência e o $i$-ésimo laboratório no mesmo ponto de rotação é dado por $\beta_{i} \sigma_{x_{j}}^{2}$, enquanto que as covariâncias entre as observações do $i$-ésimo e do $s$-ésimo laboratório no mesmo ponto de rotação é dada por $\beta_{i} \beta_{s} \sigma_{x_{j}}^{2}$, ou seja: $\operatorname{cov}\left(Y_{1 j l}, Y_{1 j q}\right)=\sigma_{x_{j}}^{2}, \operatorname{cov}\left(Y_{i j l}, Y_{i j q}\right)=\beta_{i}^{2} \sigma_{x_{j}}^{2}, \operatorname{cov}\left(Y_{1 j l}, Y_{i j q}\right)=\beta_{i} \sigma_{x_{j}}^{2}$, $\operatorname{cov}\left(Y_{i j l}, Y_{s j q}\right)=\beta_{i} \beta_{s} \sigma_{x_{j}}^{2}$, em que $i, s=2, \ldots, p, j=1, \ldots, m, l, q=1, \ldots, n_{i}$. Então,

$$
\mathbf{Y}_{1 j}=\left(Y_{1 j 1}, \ldots, Y_{1 j n_{1}}\right)^{t} \sim N_{n_{1}}\left(\boldsymbol{\mu}_{1 j}, \boldsymbol{\Sigma}_{11 j}\right)
$$

em que $\boldsymbol{\mu}_{1 j}=\mu_{x_{j}} \mathbf{1}_{n_{1}}$ e

$$
\begin{gathered}
\boldsymbol{\Sigma}_{11 j}=\left[\begin{array}{cccc}
\sigma_{x_{j}}^{2}+\sigma_{1 j}^{2} & \sigma_{x_{j}}^{2} & \cdots & \sigma_{x_{j}}^{2} \\
\sigma_{x_{j}}^{2} & \sigma_{x_{j}}^{2}+\sigma_{1 j}^{2} & \cdots & \sigma_{x_{j}}^{2} \\
\vdots & \vdots & \ddots & \vdots \\
\sigma_{x_{j}}^{2} & \sigma_{x_{j}}^{2} & \cdots & \sigma_{x_{j}}^{2}+\sigma_{1 j}^{2}
\end{array}\right]=\sigma_{1 j}^{2} \mathbf{I}_{n_{1}}+\sigma_{x_{j}}^{2} \mathbf{1}_{n_{1}} \mathbf{1}_{n_{1}}^{t}, \\
\mathbf{Y}_{i j}=\left(Y_{i j 1}, \ldots, Y_{i j n_{i}}\right)^{t} \sim N_{n_{i}}\left(\boldsymbol{\mu}_{i j}, \boldsymbol{\Sigma}_{i i j}\right)
\end{gathered}
$$


onde $\boldsymbol{\mu}_{i j}=\left(\alpha_{i}+\beta_{i} \mu_{x_{j}}\right) \mathbf{1}_{n_{i}} \mathrm{e}$

$$
\boldsymbol{\Sigma}_{i i j}=\left[\begin{array}{cccc}
\beta_{i}^{2} \sigma_{x_{j}}^{2}+\sigma_{i j}^{2} & \beta_{i}^{2} \sigma_{x_{j}}^{2} & \cdots & \beta_{i}^{2} \sigma_{x_{j}}^{2} \\
\beta_{i}^{2} \sigma_{x_{j}}^{2} & \beta_{i}^{2} \sigma_{x_{j}}^{2}+\sigma_{i j}^{2} & \cdots & \beta_{i}^{2} \sigma_{x_{j}}^{2} \\
\vdots & \vdots & \ddots & \vdots \\
\beta_{i}^{2} \sigma_{x_{j}}^{2} & \beta_{i}^{2} \sigma_{x_{j}}^{2} & \cdots & \beta_{i}^{2} \sigma_{x_{j}}^{2}+\sigma_{i j}^{2}
\end{array}\right]=\sigma_{i j}^{2} \mathbf{I}_{n_{i}}+\beta_{i}^{2} \sigma_{x_{j}}^{2} \mathbf{1}_{n_{i}} \mathbf{1}_{n_{i}}^{t}
$$

$\mathbf{I}_{n_{i}}$ e $\mathbf{1}_{n_{i}}$ denotam respectivamente, a matriz de identidade de ordem $n_{i}$ e um vetor composto por $n_{i}$ um's, $i=2, \ldots p, j=1, \ldots m$.

Observe que $\mathbf{Y}_{1 j}$ representa as medições da potência do motor pelo laboratório de referência no $j$-ésimo ponto de rotação e $\mathbf{Y}_{i j}$ as medições do $i$-ésimo laboratório no j-ésimo ponto de rotação.

Considere

$$
\mathbf{Y}_{j}=\left(\mathbf{Y}_{1 j}^{t}, \ldots, \mathbf{Y}_{p j}^{t}\right)^{t} \sim N_{n}\left(\boldsymbol{\mu}_{j}, \boldsymbol{\Sigma}_{j}\right)
$$

em que $n=\sum_{i=1}^{p} n_{i}, \boldsymbol{\mu}_{j}=\left(\boldsymbol{\mu}_{1 j}^{t}, \ldots, \boldsymbol{\mu}_{p j}^{t}\right)^{t}=\boldsymbol{\alpha}+\mu_{x j} \boldsymbol{\beta}, \operatorname{com} \boldsymbol{\alpha}=\left(\mathbf{0}_{n_{1}}^{t}, \alpha_{2} \mathbf{1}_{n_{2}}^{t}, \ldots, \alpha_{p} \mathbf{1}_{n_{p}}^{t}\right)^{t}, \boldsymbol{\beta}=$ $\left(\mathbf{1}_{n_{1}}^{t}, \beta_{2} \mathbf{1}_{n_{2}}^{t}, \ldots, \beta_{p} \mathbf{1}_{n_{p}}^{t}\right)^{t}, \mathbf{0}_{n_{1}}$ denotando um vetor composto por $n_{1}$ zeros e

$$
\boldsymbol{\Sigma}_{\boldsymbol{j}}=\left[\begin{array}{cccc}
\Sigma_{11 j} & \Sigma_{12 j} & \cdots & \Sigma_{1 p j} \\
\Sigma_{21 j} & \Sigma_{22 j} & \cdots & \Sigma_{2 p j} \\
\vdots & \vdots & \ddots & \vdots \\
\Sigma_{p 1 j} & \Sigma_{p 2 j} & \cdots & \Sigma_{p p j}
\end{array}\right]=\mathbf{D}\left(\boldsymbol{\sigma}_{j}^{2}\right)+\sigma_{x_{j}}^{2} \boldsymbol{\beta} \boldsymbol{\beta}^{t}
$$

uma matriz simétrica com $\Sigma_{11 j}$ e $\Sigma_{i i j}$ como visto em (3) e (4) respectivamente,

$$
\Sigma_{1 i j}=\beta_{i} \sigma_{x_{j}}^{2} J_{n_{1} n_{i}}
$$

$\mathrm{e}$

$$
\Sigma_{i l j}=\beta_{i} \beta_{l} \sigma_{x j}^{2} J_{n_{i} n_{l}}
$$

em que $J_{n_{i} n_{l}}$ é uma matriz $n_{i} \times n_{l}$ de uns, com $i=2, \ldots, p, l=i+1, \ldots, p, \sigma_{j}^{2}=$ 
$\left(\sigma_{1 j}^{2} \mathbf{1}_{n_{1}}^{t}, \ldots, \sigma_{p j}^{2} \mathbf{1}_{n_{p}}^{t}\right)^{t}$ e $\mathbf{D}(\mathbf{a})$ denotando uma matriz diagonal com os elementos da diagonal dados pelos elementos do vetor a.

Assim, temos:

$$
\mathbf{f}_{\mathbf{y}_{j}}\left(\mathbf{y}_{j}\right)=\frac{1}{(2 \pi)^{\frac{n}{2}}}\left|\boldsymbol{\Sigma}_{j}\right|^{-\frac{1}{2}} \exp \left[-\frac{1}{2}\left(\mathbf{y}_{j}-\boldsymbol{\mu}_{j}\right)^{t} \boldsymbol{\Sigma}_{j}^{-1}\left(\mathbf{y}_{j}-\boldsymbol{\mu}_{j}\right)\right]
$$

para $j=1, \ldots, m$, sendo $\left|\boldsymbol{\Sigma}_{j}\right|=a_{j}\left|\mathbf{D}\left(\boldsymbol{\sigma}_{j}^{2}\right)\right|, a_{j}=1+\sigma_{x_{j}}^{2} \boldsymbol{\beta}^{t} \mathbf{D}^{-1}\left(\boldsymbol{\sigma}_{j}^{2}\right) \boldsymbol{\beta}$.

Chamando a forma quadrática de,

$$
Q_{j}=\left(\mathbf{y}_{j}-\boldsymbol{\mu}_{j}\right)^{t} \boldsymbol{\Sigma}_{j}^{-1}\left(\mathbf{y}_{j}-\boldsymbol{\mu}_{j}\right)
$$

após alguns cálculos, temos que $Q_{j}$ pode ser escrita como:

$$
\overbrace{\left(\mathbf{Y}_{2 j}^{*}-\boldsymbol{\mu}_{2 j}^{*}\right)^{t} \mathbf{D}^{-1}\left(\boldsymbol{\sigma}_{2 j}^{2 *}\right)\left(\mathbf{Y}_{2 j}^{*}-\boldsymbol{\mu}_{2 j}^{*}\right)}^{Q_{1 j}}+\overbrace{\left(\mathbf{Y}_{1 j}-\boldsymbol{\mu}_{1 j}\right)^{t} \mathbf{D}^{-1}\left(\sigma_{1_{j}}^{2} \mathbf{1}_{n_{1}}\right)\left(\mathbf{Y}_{1 j}-\boldsymbol{\mu}_{1 j}\right)}^{Q_{2 j}}-
$$

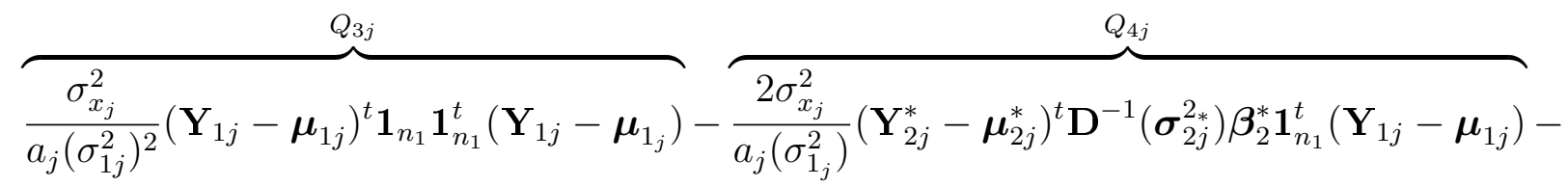

$$
\overbrace{\frac{\sigma_{x_{j}}^{2}}{a_{j}}\left(\mathbf{Y}_{2 j}^{*}-\boldsymbol{\mu}_{2 j}^{*}\right)^{t} \mathbf{D}^{-1}\left(\boldsymbol{\sigma}_{2 j}^{2 *}\right) \boldsymbol{\beta}_{2}^{*} \boldsymbol{\beta}_{2}^{* t} \mathbf{D}^{-1}\left(\boldsymbol{\sigma}_{2 j}^{2 *}\right)\left(\mathbf{Y}_{2 j}^{*}-\boldsymbol{\mu}_{2 j}^{*}\right)}^{Q_{5 j}},
$$

em que $\mathbf{Y}_{2 j}^{*}=\left(\mathbf{Y}_{2 j}^{t}, \ldots, \mathbf{Y}_{p j}^{t}\right)^{t}, \boldsymbol{\mu}_{2 j}^{*}=\left(\boldsymbol{\mu}_{2 j}^{t}, \ldots, \boldsymbol{\mu}_{p j}^{t}\right)^{t}, \boldsymbol{\beta}_{2}^{*}=\left(\beta_{2} \mathbf{1}_{n_{2}}^{t}, \ldots, \beta_{p j} \mathbf{1}_{n_{p}}^{t}\right)^{t}$ e $\boldsymbol{\sigma}_{2 j}^{2_{*}}=$ $\left(\sigma_{2 j}^{2} \mathbf{1}_{n_{2}}^{t}, \ldots, \sigma_{p j}^{2} \mathbf{1}_{n_{p}}^{t}\right)^{t}$.

As expressões de $Q_{1 j}$ a $Q_{5 j}$, que daqui para frente serão indicadas simplesmente por $Q_{j}=Q_{1 j}+Q_{2 j}+Q_{3 j}+Q_{4 j}+Q_{5 j}$, podem ser simplificadas e reescritas a fim de facilitar nossos cálculos futuros de derivadas. 
Seja $\mathbf{Y}=\left(\mathbf{Y}_{1}^{t}, \ldots, \mathbf{Y}_{m}^{t}\right)^{t}$. Da equação (6), temos

$$
f_{\mathbf{y}}(\mathbf{y})=\prod_{j=1}^{m} f_{\mathbf{y}_{j}}\left(\mathbf{y}_{j}\right)=(2 \pi)^{-\frac{m n}{2}}\left(\prod_{j=1}^{m}\left|\boldsymbol{\Sigma}_{j}\right|^{-\frac{1}{2}}\right) \exp \left[-\frac{1}{2} \sum_{j=1}^{m}\left(\mathbf{y}_{j}-\boldsymbol{\mu}_{j}\right)^{t} \boldsymbol{\Sigma}_{j}^{-1}\left(\mathbf{y}_{j}-\boldsymbol{\mu}_{j}\right)\right] .
$$

Tomando o logarítmo da função 7 , temos que a função log-verossimilhança é dada por:

$$
L(\boldsymbol{\theta})=\log \left[f_{\mathbf{y}}(\mathbf{y})\right]=-\frac{m n}{2}[\log 2 \pi]-\frac{1}{2} \sum_{j=1}^{m} \log \left|\boldsymbol{\Sigma}_{j}\right|-\frac{1}{2} \sum_{j=1}^{m}\left(\mathbf{y}_{j}-\boldsymbol{\mu}_{j}\right)^{t} \boldsymbol{\Sigma}_{j}^{-1}\left(\mathbf{y}_{j}-\boldsymbol{\mu}_{j}\right)
$$

em que $\boldsymbol{\theta}=\left(\mu_{x 1}, \ldots, \mu_{x m}, \alpha_{2}, \ldots, \alpha_{p}, \beta_{2}, \ldots, \beta_{p}\right)$.

A seguir, apresentaremos a função score, que será utilizada na obtenção das estatísticas do teste.

\section{$2.1 \quad$ Função escore}

A função escore é dada por: $U(\boldsymbol{\theta})=\frac{\partial L(\boldsymbol{\theta})}{\partial \boldsymbol{\theta}}=\left(U\left(\boldsymbol{\theta}_{1}\right)^{t}, U\left(\boldsymbol{\theta}_{2}\right)^{t}, U\left(\boldsymbol{\theta}_{3}\right)^{t}\right)^{t}$, em que, $\boldsymbol{\theta}=\left(\boldsymbol{\theta}_{1}^{t}, \boldsymbol{\theta}_{2}^{t}, \boldsymbol{\theta}_{3}^{t}\right)^{t} \mathrm{com}, \boldsymbol{\theta}_{1}=\left(\mu_{x 1}, \ldots, \mu_{x m}\right)^{t}, \boldsymbol{\theta}_{2}=\left(\alpha_{2}, \ldots, \alpha_{p}\right)^{t}, \boldsymbol{\theta}_{3}=\left(\beta_{2}, \ldots, \beta_{p}\right)^{t} \mathrm{e}$

$$
\begin{aligned}
& U\left(\boldsymbol{\theta}_{1}\right)=-\frac{1}{2} \frac{\partial Q_{j}}{\partial \boldsymbol{\theta}_{1}}, \\
& U\left(\boldsymbol{\theta}_{2}\right)=-\frac{1}{2} \sum_{j=1}^{m} \frac{\partial Q_{j}}{\partial \boldsymbol{\theta}_{2}}, \\
& U\left(\boldsymbol{\theta}_{3}\right)=-\frac{1}{2} \sum_{j=1}^{m} \frac{\partial \log \left|\boldsymbol{\Sigma}_{j}\right|}{\partial \boldsymbol{\theta}_{3}}-\frac{1}{2} \sum_{j=1}^{m} \frac{\partial Q_{j}}{\partial \boldsymbol{\theta}_{3}} .
\end{aligned}
$$

Em seguida, apresentaremos as derivadas primeiras de $\log \left|\Sigma_{j}\right|$ e $Q_{j}$ com relação aos parâmetros. Então, notando que:

$$
\frac{\partial a_{j}}{\partial \alpha_{i}}=\frac{\partial a_{j}}{\partial \mu_{x_{j}}}=0, \frac{\partial a_{j}}{\partial \beta_{i}}=\frac{2 n_{i} \beta_{i} \sigma_{x j}^{2}}{\sigma_{i j}^{2}}, \frac{\partial \log a_{j}}{\partial \beta_{i}}=\frac{2 n_{i} \beta_{i} \sigma_{x j}^{2}}{a_{j} \sigma_{i j}^{2}}
$$

e após algumas manipulações algébricas, temos que:

I) Derivada primeira de $\log \left|\Sigma_{j}\right| \operatorname{com}$ relação ao parâmetro $\beta_{i}$, 
$\frac{\partial \log \left|\Sigma_{j}\right|}{\partial \beta_{i}}=\frac{1}{a_{j}}\left(\frac{2 n_{i} \beta_{i} \sigma_{x j}^{2}}{\sigma_{i j}^{2}}\right), i=2, \ldots, p$.

II) Derivadas primeiras de $Q_{j}$ com relação aos parâmetros

a) $\frac{\partial Q_{j}}{\partial \mu_{x_{j}}}=\left[\frac{2}{a_{j}}\right]\left[\frac{\mu_{x_{j}}\left(a_{j}-1\right)}{\sigma_{x_{j}}^{2}}-\frac{\sum_{k=1}^{n 1} y_{1 j k}}{\sigma_{1 j}^{2}}-\sum_{i=2}^{p} \frac{\beta_{i}}{\sigma_{i j}^{2}}\left(\sum_{k=1}^{n_{i}} y_{i j k}-\alpha_{i} n_{i}\right)\right], j=1, \ldots, m$.

b) $\frac{\partial Q_{j}}{\partial \mu_{x_{h}}}=0, j, h=1, \ldots, m$, para $h \neq j$.

c) $\frac{\partial Q_{j}}{\partial \alpha_{i}}=\frac{2}{\sigma_{i j}^{2}}\left\{\frac{n_{i} \beta_{i} \sigma_{x_{j}}^{2} M_{j}}{a_{j}}+n_{i} \alpha_{i}-\sum_{k=1}^{n_{i}} y_{i j k}\right\}$, para $j=1, \ldots, m$ e $i=2, \ldots, p$.

d) $\frac{\partial Q_{j}}{\partial \beta_{i}}=M_{j} \frac{\sigma_{x j}^{2}}{a_{j}}\left[\frac{\partial Q_{j}}{\partial \alpha_{i}}\right]$ onde, $j=1, \ldots, m$ e $i=2, \ldots, p$.

Na sequência, apresentaremos a matriz de informação observada.

\subsection{Matriz de informação observada}

A matriz de informação observada é dada por $I(\boldsymbol{\theta})=-\frac{\partial^{2} L(\boldsymbol{\theta})}{\partial \boldsymbol{\theta} \partial \boldsymbol{\theta}^{t}}$, em que, $\boldsymbol{\theta}=$ $\left(\boldsymbol{\theta}_{1}^{t}, \boldsymbol{\theta}_{2}^{t}, \boldsymbol{\theta}_{3}^{t}\right)^{t} \operatorname{com}, \boldsymbol{\theta}_{1}^{t}=\left(\mu_{x 1}, \ldots, \mu_{x m}\right)^{t}, \boldsymbol{\theta}_{2}^{t}=\left(\alpha_{2}, \ldots, \alpha_{p}\right)^{t}, \boldsymbol{\theta}_{3}^{t}=\left(\beta_{2}, \ldots, \beta_{p}\right)^{t}$ e portanto,

$$
I(\boldsymbol{\theta})=\left[\begin{array}{ccc}
{ }^{I_{\boldsymbol{\theta}_{1}} \boldsymbol{\theta}_{1}} & { }^{I_{\boldsymbol{\theta}_{1}} \boldsymbol{\theta}_{2}} & { }^{I} \boldsymbol{\theta}_{\boldsymbol{\theta}_{1} \boldsymbol{\theta}_{3}} \\
I_{\boldsymbol{\theta}_{2} \boldsymbol{\theta}_{1}} & { }^{I_{\boldsymbol{\theta}_{2}} \boldsymbol{\theta}_{2}} & { }^{I_{\boldsymbol{\theta}_{2}} \boldsymbol{\theta}_{3}} \\
{ }^{I_{\boldsymbol{\theta}_{3}} \boldsymbol{\theta}_{1}} & { }^{I_{\boldsymbol{\theta}_{3}} \boldsymbol{\theta}_{2}} & { }^{I} \boldsymbol{\theta}_{3} \boldsymbol{\theta}_{3}
\end{array}\right],
$$

em que,

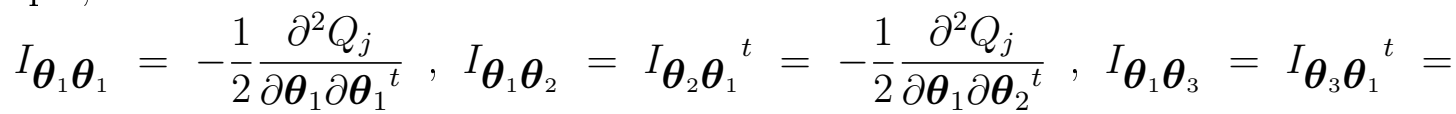

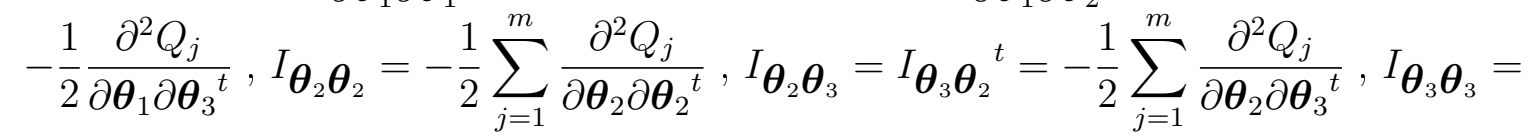

$$
\begin{aligned}
& -\frac{1}{2} \sum_{j=1}^{m} \frac{\partial^{2} \log \left|\boldsymbol{\Sigma}_{j}\right|}{\partial \boldsymbol{\theta}_{3} \partial \boldsymbol{\theta}_{3}{ }^{t}}-\frac{1}{2} \sum_{j=1}^{m} \frac{\partial^{2} Q_{j}}{\partial \boldsymbol{\theta}_{3} \partial \boldsymbol{\theta}_{3}{ }^{t}} \text {. }
\end{aligned}
$$

Desta forma, 


$$
I(\boldsymbol{\theta})=\left[\begin{array}{ccccccccc}
I_{\mu_{x 1} \mu_{x 1}} & \cdots & I_{\mu_{x 1} \mu_{x m}} & I_{\mu_{x 1} \alpha_{2}} & \cdots & I_{\mu_{x 1} \alpha_{p}} & I_{\mu_{x 1} \beta_{2}} & \cdots & I_{\mu_{x 1} \beta_{p}} \\
\vdots & \ddots & \vdots & \vdots & \ddots & \vdots & \vdots & \ddots & \vdots \\
I_{\mu_{x m} \mu_{x 1}} & \cdots & I_{\mu_{x m} \mu_{x m}} & I_{\mu_{x m} \alpha_{2}} & \cdots & I_{\mu_{x m} \alpha_{p}} & I_{\mu_{x m} \beta_{2}} & \cdots & I_{\mu_{x m} \beta_{p}} \\
I_{\alpha_{2} \mu_{x 1}} & \cdots & I_{\alpha_{2} \mu_{x m}} & I_{\alpha_{2} \alpha_{2}} & \cdots & I_{\alpha_{2} \alpha_{p}} & I_{\alpha_{2} \beta_{2}} & \cdots & I_{\alpha_{2} \beta_{p}} \\
\vdots & \ddots & \vdots & \vdots & \ddots & \vdots & \vdots & \ddots & \vdots \\
I_{\alpha_{p} \mu_{x 1}} & \cdots & I_{\alpha_{p} \mu_{x m}} & I_{\alpha_{p} \alpha_{2}} & \cdots & I_{\alpha_{p} \alpha_{p}} & I_{\alpha_{p} \beta_{2}} & \cdots & I_{\alpha_{p} \beta_{p}} \\
I_{\beta_{2} \mu_{x 1}} & \cdots & I_{\beta_{2} \mu_{x m}} & I_{\beta_{2} \alpha_{2}} & \cdots & I_{\beta_{2} \alpha_{p}} & I_{\beta_{2} \beta_{2}} & \cdots & I_{\beta_{2} \beta_{p}} \\
\vdots & \ddots & \vdots & \vdots & \ddots & \vdots & \vdots & \ddots & \vdots \\
I_{\beta_{p} \mu_{x 1}} & \cdots & I_{\beta_{p} \mu_{x m}} & I_{\beta_{p} \alpha_{2}} & \cdots & I_{\beta_{p} \alpha_{p}} & I_{\beta_{p} \beta_{2}} & \cdots & I_{\beta_{p} \beta_{p}}
\end{array}\right]
$$

em que,

$$
I_{\theta_{l} \theta_{h}}=-\frac{\partial^{2} L(\boldsymbol{\theta})}{\partial \theta_{l} \partial \theta_{h}} .
$$

Apresentaremos a seguir as derivadas de segunda ordem do $\log \left|\Sigma_{j}\right|$ e $Q_{j}$ em relação aos parâmetros.

I) Derivadas de segunda ordem de $\log \left|\Sigma_{j}\right|$ em relação ao parâmetro $\beta_{i}, i=2, \ldots, p$.
a) $\frac{\partial^{2} \log \left|\Sigma_{j}\right|}{\partial \beta_{i}}=\frac{2 n_{i} \sigma_{x j}^{2}}{a_{j} \sigma_{i j}^{2}}\left[1-\frac{2 n_{i} \beta_{i}^{2} \sigma_{x j}^{2}}{a_{j} \sigma_{i j}^{2}}\right]$, para $i=2, \ldots, p$.
b) $\frac{\partial^{2} \log \left|\Sigma_{j}\right|}{\partial \beta_{i} \partial \beta_{l}}=-\frac{4 n_{i} n_{l} \beta_{i} \beta_{l}\left(\sigma_{x j}^{2}\right)^{2}}{a_{j}^{2} \sigma_{i j}^{2} \sigma_{l j}^{2}}$, para $i, l=2, \ldots, p$ e $i \neq l$.

Sejam:

$M_{j}=\frac{\mu_{x_{j}}}{\sigma_{x_{j}}^{2}}+\frac{\sum_{k=1}^{n 1} y_{1 j k}}{\sigma_{1 j}^{2}}+\sum_{i=2}^{p} \frac{\beta_{i}}{\sigma_{i j}^{2}}\left(\sum_{k=1}^{n i} y_{i j k}-n_{i} \alpha_{i}\right), a_{j}$ como definido em $(6), D_{i j}=$ $\mathbf{1}_{n i}^{t}\left(\mathbf{Y}_{i j}-\alpha_{i} \mathbf{1}_{n i}\right)=\sum_{k=1}^{n i} y_{i j k}-n_{i} \alpha_{i} \operatorname{com} j=1, \ldots, m$ e $i=2, \ldots, p$.

Assim,

$\frac{\partial M_{j}}{\partial \mu_{x j}}=\frac{1}{\sigma_{x j}^{2}}, \frac{\partial M_{j}}{\partial \alpha_{i}}=-\frac{n_{i} \beta_{i}}{\sigma_{i j}^{2}}$ e $\frac{\partial M_{j}}{\partial \beta_{i}}=\frac{\sum_{k=1}^{n_{i}} y_{i j k}-n_{i} \alpha_{i}}{\sigma_{i j}^{2}}$.

E então, após algumas manipulações algébricas, temos:

II) Derivadas de segunda ordem de $Q_{j}$ em relação aos parâmetros 

a) $\frac{\partial^{2} Q_{j}}{\partial \mu_{x j}}=2 \frac{a_{j}-1}{a_{j} \sigma_{x j}^{2}}, j=1, \ldots, m$
b) $\frac{\partial^{2} Q_{j}}{\partial \mu_{x h}}=0, j, h=1, \ldots, m, j \neq h$,
c) $\frac{\partial^{2} Q_{j}}{\partial \mu_{x j} \partial \mu_{x h}}=0, j, h=1, \ldots, m, j \neq h$,
d) $\frac{\partial^{2} Q_{j}}{\partial \mu_{x j} \partial \alpha_{i}}=2 \frac{n_{i} \beta_{i}}{a_{j} \sigma_{i j}^{2}}$, para $j=1, \ldots, m$ e $i=2, \ldots, p$,
e) $\frac{\partial^{2} Q_{j}}{\partial \mu_{x h} \partial \alpha_{i}}=0, j \neq h=1, \ldots, m$
f) $\frac{\partial^{2} Q_{j}}{\partial \mu_{x j} \partial \beta_{i}}=-\frac{2}{\sigma_{i j}^{2} a_{j}}\left[\sum_{k=1}^{n i} y_{i j k}-\frac{n_{i}}{a_{j}}\left(\alpha_{i} a_{j}+2 \beta_{i} \sigma_{x j}^{2} M_{j}\right)\right]$, para $j=1, \ldots, m$ e $i=$
$2, \ldots, p$
g) $\frac{\partial^{2} Q_{j}}{\partial \mu_{x h} \partial \beta_{i}}=0, h \neq j=1, \ldots, m$ e $i=2, \ldots, p$,
h) $\frac{\partial^{2} Q_{j}}{\partial \alpha_{i}}=2 \frac{n_{i}}{\sigma_{i j}^{2}}\left(1-\frac{n_{i} \beta_{i}^{2} \sigma_{x j}^{2}}{\sigma_{i j}^{2} a_{j}}\right)$, para $i=2, \ldots, p$,
i) $\frac{\partial^{2} Q_{j}}{\partial \alpha_{i} \partial \alpha_{l}}=-2 \frac{n_{i} n_{l} \beta_{i} \beta_{l} \sigma_{x j}^{2}}{a_{j} \sigma_{i j}^{2} \sigma_{l j}^{2}}$, para $i, l=2, \ldots, p$ e $i \neq l$,
j) $\frac{\partial^{2} Q_{j}}{\partial \alpha_{i} \partial \beta_{i}}=2 \frac{n_{i} \sigma_{x j}^{2}}{\sigma_{i j}^{2} a_{j}}\left[M_{j}-\frac{\beta_{i}}{\sigma_{i j}^{2}}\left[\frac{2 n_{i} \beta_{i} \sigma_{x j}^{2} M_{j}}{a_{j}}-D_{i j}\right]\right]$, para $i=2, \ldots, p$,
l) $\frac{\partial^{2} Q_{j}}{\partial \alpha_{i} \partial \beta_{l}}=2 \frac{n_{i} \beta_{i} \sigma_{x j}^{2}}{\sigma_{i j}^{2} \sigma_{l j}^{2} a_{j}}\left[D_{l j}-\frac{2 n_{l} \beta_{l} \sigma_{x j}^{2} M_{j}}{a_{j}}\right]$, para $i, l=2, \ldots, p$ e $i \neq l$,
m) $\frac{\partial^{2} Q_{j}}{\partial \beta_{i}}=-2 \frac{\sigma_{x j}^{2}}{a_{j} \sigma_{i j}^{2}}\left[\frac{D_{i j}^{2}}{\sigma_{i j}^{2}}-\frac{n_{i} \sigma_{x j}^{2}}{a_{j}}\left[M_{j}^{2}\left(1-\frac{4 n_{i} \sigma_{x j}^{2} \beta_{i}^{2}}{a_{j} \sigma_{i j}^{2}}\right)+\frac{4 M_{j} D_{i j} \beta_{i}}{\sigma_{i j}^{2}}\right]\right]$, para $i=2, \ldots, p$,
n) $\frac{\partial^{2} Q_{j}}{\partial \beta_{i} \partial \beta_{l}}=-2 \frac{\sigma_{x j}^{2}}{\sigma_{i j}^{2} \sigma_{l j}^{2} a_{j}}\left[D_{i j} D_{l j}+\frac{2 \sigma_{x j}^{2}}{a_{j}}\left[n_{l} \beta_{l}\left(\frac{2 n_{i} \beta_{i} \sigma_{x j}^{2} M_{j}^{2}}{a_{j}}\right)-n_{i} \beta_{i} M_{j} D_{l j}-n_{l} \beta_{l} M_{j} D_{i j}\right]\right]$, para $i, l=2, \ldots, p$ e $i \neq l$.

Então, pode-se notar que:
a) $I_{\mu_{x j} \mu_{x j}}=\frac{a_{j}-1}{a_{j} \sigma_{x j}^{2}}$, para $j=1, \ldots, m$,
b) $I_{\mu_{x j} \mu_{x h}}=0$, para $j, h=1, \ldots, m, j \neq h$,
c) $I_{\mu_{x j} \alpha_{i}}=\frac{n_{i} \beta_{i}}{a_{j} \sigma_{i j}^{2}}$, para $j=1, \ldots, m$ e $i=2, \ldots, p$,
d) $I_{\mu_{x j} \beta_{i}}=\frac{1}{\sigma_{i j}^{2} a_{j}}\left[\frac{2 n_{i} \beta_{i} \sigma_{x j}^{2} M_{j}}{a_{j}}-D_{i j}\right]$, para $j=1, \ldots, m$ e $i=2, \ldots, p$,
e) $I_{\alpha_{i} \alpha_{i}}=\sum_{j=1}^{m} \frac{n_{i}}{\sigma_{i j}^{2}}\left(1-\frac{n_{i} \beta_{i}^{2} \sigma_{x j}^{2}}{\sigma_{i j}^{2} a_{j}}\right)$, para $i=2, \ldots, p$, 
f) $I_{\alpha_{i} \alpha_{l}}=-\sum_{j=1}^{m} \frac{n_{i} n_{l} \beta_{i} \beta_{l} \sigma_{x j}^{2}}{a_{j} \sigma_{i j}^{2} \sigma_{l j}^{2}}$, para $i, l=2, \ldots, p$ e $i \neq l$,

g) $I_{\alpha_{i} \beta_{i}}=\sum_{j=1}^{m} \frac{n_{i} \sigma_{x j}^{2}}{\sigma_{i j}^{2} a_{j}}\left[M_{j}-\frac{\beta_{i}}{\sigma_{i j}^{2}}\left[\frac{2 n_{i} \beta_{i} \sigma_{x j}^{2} M_{j}}{a_{j}}-D_{i j}\right]\right]$, para $i=2, \ldots, p$,

h) $I_{\alpha_{i} \beta_{l}}=\sum_{j=1}^{m} \frac{n_{i} \beta_{i} \sigma_{x j}^{2}}{\sigma_{i j}^{2} \sigma_{l j}^{2} a_{j}}\left[D_{l j}-\frac{2 n_{l} \beta_{l} \sigma_{x j}^{2} M_{j}}{a_{j}}\right]$, para $i, l=2, \ldots, p$ e $i \neq l$,

i) $I_{\beta_{i} \beta_{i}}=\sum_{j=1}^{m} \frac{\sigma_{x j}^{2}}{a_{j} \sigma_{i j}^{2}}\left[n_{i}-\frac{D_{i j}^{2}}{\sigma_{i j}^{2}}+\frac{n_{i} \sigma_{x j}^{2}}{a_{j}}\left[M_{j}^{2}\left(1-\frac{4 n_{i} \sigma_{x j}^{2} \beta_{i}^{2}}{a_{j} \sigma_{i j}^{2}}\right)-\frac{2 n_{i} \beta_{i}^{2}}{\sigma_{i j}^{2}}+\frac{4 M_{j} \beta_{i} D_{i j}}{\sigma_{i j}^{2}}\right]\right]$,

para $i=2, \ldots, p$,

j) $I_{\beta_{i} \beta_{l}}=-\sum_{j=1}^{m} \frac{\sigma_{x j}^{2}}{\sigma_{i j}^{2} \sigma_{l j}^{2} a_{j}}\left[D_{i j} D_{l j}+\frac{2 \sigma_{x j}^{2}}{a_{j}}\left[n_{i} n_{l} \beta_{i} \beta_{l}\left(\frac{2 \sigma_{x j}^{2} M_{j}^{2}}{a_{j}}+1\right) n_{i} \beta_{i} M_{j} D_{l j}-n_{l} \beta_{l} M_{j} D_{i j}\right]\right]$, para $i, l=2, \ldots, p$ e $i \neq l$.

Na próxima subseção, apresentaremos uma metodologia para a estimação dos parâmetros do modelo e desenvolveremos os testes de hipóteses de interesse.

\subsection{Estimação dos parâmetros e testes de hipóteses}

Nesta Seção estamos interessados em obter os valores das estimativas dos parâmetros do modelo. Como não conseguimos explicitar tais estimativas, utilizaremos o algoritmo EM, descrito abaixo, muito usado como uma alternativa para obtenção das estimativas de máxima verossimilhança dos parâmetros.

\subsubsection{Algoritmo EM}

O algoritmo EM (Expectation-Maximization) é um dos algoritmos mais usados na Estatística (McLachlan and Krishnan(1997)) para a obtenção de estimativas de máxima verossimilhança (EMV). É um procedimento iterativo eficiente para a obtenção da estimativa de máxima verossimilhança na presença de dados perdidos ou incompletos ou em modelos com variáveis latentes. Foi proposto primeiro por Dempster (1977), com exemplos e aplicações, mas outros autores já haviam trabalhado com tal algoritmo. 
A referência mais antiga da literatura para um algoritmo do tipo EM é Newcomb(1886), que considera a estimação dos parâmetros da mistura de duas normais univariadas. McKendreick(1926) apresenta uma aplicação médica para um método no "espírito" do algoritmo EM. Hartley(1958) e Buck(1960) também nos dão outros exemplos de aplicações. Vários artigos tratam de aplicações do algoritmo EM em modelos markovianos.

Oliveira(2001) utiliza o algoritmo em um modelo de calibração comparativa para comparar a eficiência dos instrumentos de medição em diferentes alturas de árvores. Zavala(2001) apresenta um estudo do algoritmo EM nos modelos de regressão lineares mistos com erros nas variáveis. Lima (1996) trata dos modelos de calibração absoluta com erros nas variáveis. Bolfarine \& Rojas (1995) utilizam o algoritmo em um modelo de calibração estrutural. Labra, Aoki \& Bolfarine (2005) tratam da influência local em uma regressão com erro de medição com intercepto nulo sob a distribuição t de Student e para isso, fazem uso do algoritmo EM para encontrar as estimativas dos parâmetros do modelo. Um exemplo fora da área de exatas, pode ser encontrado em Chuong \& Batzoglou (2008) que utilizam o EM na área da biologia.

A metodologia consiste em acrescentar aos dados observados, alguns dados não observados ou perdidos de uma maneira que a obtenção das estimativas de MV baseada nos dados completos (observados juntamente com os não observados que foram acrescentados) seja fácil de ser obtida.

O algoritmo é facil de ser implementado, e é dividido em 2 etapas: etapa E (E-step) e etapa M (M-step).

Na etapa E, o dado incompleto é estimado utilizando esperança condicional da log-verossimilhança dos dados completos, dadas as observações e as estimativas dos parâmetros da iteração anterior.

Na etapa M, a equação obtida na etapa E é maximizada em relação aos parâmetros, obtendo assim as estimativas da etapa seguinte.

Considerando o modelo definido em (1) e (2) e os dados observados para o $j$-ésimo 
ponto de rotação, ou seja,

$$
\mathbf{Y}_{j}=\left(\mathbf{Y}_{1 j}^{t}, \ldots, \mathbf{Y}_{p j}^{t}\right)^{t} \sim N\left(\boldsymbol{\mu}_{j}, \boldsymbol{\Sigma}_{j}\right)
$$

acrescente a $\mathbf{Y}_{j}$ o dado não observado $x_{j}$.

Então os dados completos para o $j$-ésimo ponto de rotação são dados por $\mathbf{Y}_{j c}=$ $\left(x_{j}, \mathbf{Y}_{j}^{t}\right)^{t}, \operatorname{com} \mathbf{Y}_{j c} \sim N_{n+1}\left(\boldsymbol{\mu}_{j c}, \boldsymbol{\Sigma}_{j c}\right)$, onde $\boldsymbol{\mu}_{j c}=\left(\mu_{x_{j}}, \boldsymbol{\alpha}+\mu_{x_{j}} \boldsymbol{\beta}\right) \mathrm{e}$

$$
\boldsymbol{\Sigma}_{j c}=\left[\begin{array}{cc}
\sigma_{x j}^{2} & A_{12 j} \\
A_{21 j} & \Sigma j
\end{array}\right]
$$

$\operatorname{com} j=1, \ldots, m$ e $A_{12 j}^{t}=A_{21 j}$, em que $A_{12 j}=\operatorname{cov}\left(x_{j}, \mathbf{Y}_{j}\right)=\sigma_{x_{j}}^{2} \boldsymbol{\beta}^{t}$, e $\boldsymbol{\Sigma}_{j}$ e $\boldsymbol{\mu}_{j}$ como em (5).

Seja $\mathbf{Y}_{c}=\left(\mathbf{Y}_{1 c}^{t}, \ldots, \mathbf{Y}_{m c}^{t}\right)^{t}$ então,

$$
f_{\mathbf{y}_{c}}\left(\mathbf{y}_{c}\right)=\prod_{j=1}^{m}\left[\frac{1}{(2 \pi)^{\frac{n+1}{2}}}\left|\boldsymbol{\Sigma}_{j c}\right|^{-\frac{1}{2}} \exp \left[-\frac{1}{2}\left(\mathbf{Y}_{j c}-\boldsymbol{\mu}_{j c}\right)^{t} \boldsymbol{\Sigma}_{j c}^{-1}\left(\mathbf{Y}_{j c}-\boldsymbol{\mu}_{j c}\right)\right]\right]
$$

Segue que a função log-verosimilhança dos dados completos é dada por:

$$
\begin{gathered}
L_{c}(\boldsymbol{\theta})=-\frac{m(n+1)}{2} \log (2 \pi)-\frac{1}{2} \sum_{j=1}^{m} \log \left|\boldsymbol{\Sigma}_{j c}\right|-\frac{1}{2} \sum_{j=1}^{m}\left(\mathbf{Y}_{j c}-\boldsymbol{\mu}_{j c}\right)^{t} \boldsymbol{\Sigma}_{j c}^{-1}\left(\mathbf{Y}_{j c}-\boldsymbol{\mu}_{j c}\right)= \\
c t e-\frac{1}{2} \sum_{j=1}^{m}\left(\log \sigma_{x j}^{2}+\sum_{i=1}^{p} n_{i} \log \sigma_{i j}^{2}\right)-\frac{1}{2}\left[\sum_{j=1}^{m} \frac{\left(x_{j}-\mu_{x j}\right)^{2}}{\sigma_{x j}^{2}}+\sum_{j=1}^{m} \sum_{k=1}^{n 1} \frac{\left(y_{1 j k}-x_{j}\right)^{2}}{\sigma_{1 j}^{2}}\right. \\
\left.+\sum_{j=1}^{m} \sum_{i=2}^{p} \sum_{k=1}^{n i} \frac{\left(y_{i j k}-\alpha_{i}-\beta_{i} x_{j}\right)^{2}}{\sigma_{i j}^{2}}\right] .
\end{gathered}
$$

Agora podemos calcular as etapas E e M do algoritmo.

\section{Etapa E}

Queremos encontrar as expressões para $\hat{x}_{j}^{(r)}=E\left[x_{j} \mid \mathbf{Y}_{j}, \hat{\boldsymbol{\theta}}^{(r-1)}\right]$ e ${\hat{x^{2}}}_{j}^{(r)}=E\left[x_{j}^{2} \mid \mathbf{Y}_{j}, \hat{\boldsymbol{\theta}}^{(r-1)}\right]$. 
De Searle(1971) e Johnson(1998), temos que:

$$
\hat{x}_{j}^{(r)}=E\left[x_{j} \mid \mathbf{Y}_{j}, \hat{\boldsymbol{\theta}}^{(r-1)}\right]=\mu_{x j}+A_{12 j} \Sigma_{j}^{-1}\left(\mathbf{Y}_{j}-\boldsymbol{\mu}_{j}\right) .
$$

Substituindo os valores e fazendo algumas manipulações algébricas, temos que:

$$
\hat{x}_{j}^{(r)}=\frac{\sigma_{x_{j}}^{2}}{\hat{a}_{j}^{(r-1)}}\left(\frac{\hat{\mu}_{x j}^{(r-1)}}{\sigma_{x_{j}}^{2}}+\frac{1}{\sigma_{1 j}^{2}} \sum_{k=1}^{n 1} y_{1 j k}+\sum_{i=2}^{p} \frac{\hat{\beta}_{i}^{(r-1)}}{\sigma_{i j}^{2}}\left(\sum_{k=1}^{n i} y_{i j k}-n_{i} \hat{\alpha}_{i}^{(r-1)}\right)\right),
$$

em que $\hat{a}_{j}^{(r-1)}=1+\sigma_{x j}^{2}\left(\frac{n_{1}}{\sigma_{1 j}}+\sum_{i=2}^{p} \frac{n_{i} \hat{\beta}_{i}^{2(r-1)}}{\sigma_{i j}^{2}}\right)$.

Temos também que

$$
{\widehat{x^{2}}}_{j}^{(r)}=E\left[x_{j}^{2} \mid \mathbf{Y}_{j}, \hat{\boldsymbol{\theta}}^{(r-1)}\right]=\operatorname{Var}\left[x_{j} \mid \mathbf{Y}_{j}, \hat{\boldsymbol{\theta}}^{(r-1)}\right]+E\left[x_{j} \mid \mathbf{Y}_{j}, \hat{\boldsymbol{\theta}}^{(r-1)}\right]^{2} \text {. Então, novamente }
$$

após as substituições e manipulações algébricas, temos que:

$$
{\widehat{x^{2}}}_{j}^{(r)}=\left(\hat{x}_{j}^{(r)}\right)^{2}+\frac{\sigma_{x j}^{2}}{\hat{a}_{j}^{(r-1)}} \text {. }
$$

Desta forma:

$$
\begin{gathered}
E\left(L_{c}(\boldsymbol{\theta}) \mid \mathbf{Y}, \hat{\boldsymbol{\theta}}^{(r-1)}\right)= \\
\text { cte }-\frac{1}{2} \sum_{j=1}^{m}\left(\log \sigma_{x_{j}}^{2}+\sum_{i=1}^{p} n_{i} \log \sigma_{i j}^{2}\right)-\frac{1}{2}\left[\sum_{j=1}^{m}{\widehat{x_{j}^{2}}}^{(r)}\left(\frac{1}{\sigma_{x_{j}}^{2}}+\frac{n_{1}}{\sigma_{1 j}^{2}}+\sum_{i=2}^{p} \frac{n_{i} \hat{\beta}_{i}^{2(r-1)}}{\sigma_{i j}^{2}}\right)\right. \\
+\sum_{j=1}^{m} \hat{x}_{j}^{(r)}\left(-2 \frac{\hat{\mu}_{x_{j}}^{(r-1)}}{\sigma_{x_{j}}^{2}}-2 \sum_{k=1}^{n_{1}} \frac{y_{1 j k}}{\sigma_{1 j}^{2}}-2 \sum_{i=2}^{p} \sum_{k=1}^{n_{i}} \frac{y_{i j k} \hat{\beta}_{i}^{(r-1)}+\hat{\alpha}_{i}^{(r-1)} \hat{\beta}_{i}^{(r-1)}}{\sigma_{i j}^{2}}\right) \\
\left.+\sum_{j=1}^{m} \frac{\hat{\mu}_{x_{j}}^{2(r-1)}}{\sigma_{x_{j}}^{2}}+\sum_{j=1}^{m} \sum_{k=1}^{n_{1}} \frac{y_{1 j k}^{2}}{\sigma_{1 j}^{2}}+\sum_{j=1}^{m} \sum_{i=2}^{p} \sum_{k=1}^{n_{i}} \frac{\left(y_{i j k}-\hat{\alpha}_{i}^{(r-1)}\right)^{2}}{\sigma_{i j}^{2}}\right] .
\end{gathered}
$$

\section{Etapa M}

Nesta etapa estamos interessados em maximizar a esperança condicional da função de verossimilhança completa dadas as observações e as estimativas dos parâmetros da iteração anterior, ou seja, queremos encontrar os valores de $\hat{\mu}_{x j}^{(r)}, \hat{\alpha}_{i}{ }^{(r)}$ e $\hat{\beta}_{i}^{(r)}$, $i=2, \ldots, p$ e $j=1, \ldots, m$. Após calcular as derivadas e isolar os termos, chegamos a:

$$
\hat{\mu}_{x j}^{(r)}=\hat{x}_{j}^{(r)}, j=1, \ldots, m
$$




$$
\begin{gathered}
\hat{\alpha}_{i}^{(r)}=\frac{\sum_{j=1}^{m} \sum_{k=1}^{n_{i}} \frac{y_{i j k}}{\sigma_{i j}^{2}}-n_{i} \hat{\beta}_{i}^{(r)} \sum_{j=1}^{m} \frac{\hat{x}_{j}^{(r)}}{\sigma_{i j}^{2}}}{n_{i} \sum_{j=1}^{m} \frac{1}{\sigma_{i j}^{2}}}, i=2, \ldots, p, \\
\hat{\beta}_{i}^{(r)}=\frac{\frac{1}{n_{i}}\left(\left(\sum_{j=1}^{m} \frac{\hat{x}_{j}^{(r)}}{\sigma_{i j}^{2}} \sum_{k=1}^{n_{i}} y_{i j k}\right)\left(\sum_{j=1}^{m} \frac{1}{\sigma_{i j}^{2}}\right)-\left(\sum_{j=1}^{m} \sum_{k=1}^{n_{i}} \frac{y_{i j k}}{\sigma_{i j}^{2}}\right)\left(\sum_{j=1}^{m} \frac{\hat{x}_{j}^{(r)}}{\sigma_{i j}^{2}}\right)\right)}{\left(\sum_{j=1}^{m} \frac{\widehat{x}_{j}^{2}}{\sigma_{i j}^{2}}\right)\left(\sum_{j=1}^{m} \frac{1}{\sigma_{i j}^{2}}\right)-\left(\sum_{j=1}^{m} \frac{\hat{x}_{j}^{(r)}}{\sigma_{i j}^{2}}\right)^{2}},
\end{gathered}
$$

$i=2, \ldots, p$.

\subsubsection{Testes de hipóteses assintóticos}

Nesta Seção, usaremos os testes de hipóteses assintóticos de Wald e razão de verossimilhança para testar as hipóteses de interesse.

O teste de Wald é um teste paramétrico, ou seja, baseado nos parâmetros da amostra, como por exemplo média e desvio padrão; seu uso está condicionado à dimensão da amostra (pois é um teste assintótico) e à respectiva distribuição das variáveis em estudo. O teste de Wald pode ser utilizado para testar o verdadeiro valor do parâmetro com base na estimativa de amostra e também pode ser utilizado em uma grande variedade de diferentes modelos, incluindo modelos para variáveis discretas e variáveis contínuas (Fisher, 1925). Neste teste são usados os valores das estimativas de máxima verossimilhança.

O teste da razão de verossimilhança é um teste estatístico que compara o ajuste de dois modelos; baseia-se na razão de probabilidade, que expressa o número de vezes mais provável que os dados estão sob um modelo do que o outro (Rao, 1973). Este teste utiliza os valores das estimativas de máxima verossimilhança e também das estimativas de máxima verossimilhança restritos a hipótese que estamos trabalhando. Normalmente, o teste de Wald e o teste de razão de verossimilhança dão conclusões muito semelhantes (pois são assintoticamente equivalentes), mas muito raramente, eles discordam o suficiente para levar a conclusões diferentes.

No nosso modelo, as observações não são identicamente distribuídas para cada um dos patamares. O parâmetro $\mu_{x_{j}}$ é específico do patamar $j, j=1, \ldots, m$, enquanto 
que os parâmetros $\alpha_{i}$ e $\beta_{i}, i=2, \ldots p$, são comuns a todos os patamares. O número de observações em cada patamar é dado por $n=\sum_{i=1}^{p} n_{i}$. Desta forma, podemos mostrar a normalidade assintótica do EMV como um caso paticular do Teorema 5.1 apresentado em Russo (2006) que pode ser encontrado no Apêndice A. Com este resultado e assumindo válidas as condições de regularidade do Teorema 5.1, podemos testar $H_{0}: \mathbf{h}(\boldsymbol{\theta})=0$ vs. $H_{1}: \mathbf{h}(\boldsymbol{\theta}) \neq 0$, em que $\mathbf{h}(\boldsymbol{\theta})=\left(h_{1}, \ldots, h_{r}\right)^{t}: \mathbb{R}^{k} \rightarrow \mathbb{R}^{r}(r \leq k)$ é tal que

(i) Existem $k-r$ funções adicionais $h^{r+1}(\boldsymbol{\theta}), \ldots, h^{k}(\boldsymbol{\theta})$ tal que as relações inversas $\theta_{1}\left(\mathbf{h}^{*}\right), \ldots, \theta_{k}\left(\mathbf{h}^{*}\right)$ existem, com $\mathbf{h}^{*}=\left(h_{1}(\boldsymbol{\theta}), \ldots, h_{k}(\boldsymbol{\theta})\right)$.

(ii) as derivadas parciais de primeira e segunda ordem de $h_{1}(\boldsymbol{\theta}), \ldots, h_{k}(\boldsymbol{\theta})$ são funções uniformemente contínuas e limitadas de $\boldsymbol{\theta}$.

(iii) o maior limite inferior do valor absoluto do jacobiano $\frac{\partial\left(h_{1}, \ldots, h_{k}\right)^{\prime}}{\partial\left(\theta_{1}, \ldots, \theta_{k}\right)}$ é positivo.

Seja a matriz $\mathbf{H}(\boldsymbol{\theta})=\frac{\partial \mathbf{h}(\boldsymbol{\theta})^{\prime}}{\partial \boldsymbol{\theta}}$ contínua em $\boldsymbol{\theta}$ com posto r. Sejam $\hat{\boldsymbol{\theta}}$ o EMV de $\boldsymbol{\theta}$ e $\overline{\boldsymbol{\theta}}$ o estimador de máxima verossimilhança restrito à $H_{0}$ e seja,

Estatística de Wald

$$
\text { - } Q_{W}=\mathbf{h}(\hat{\boldsymbol{\theta}})^{t}\left[\mathbf{H}(\hat{\boldsymbol{\theta}})^{t}[I(\hat{\boldsymbol{\theta}})]^{-1} \mathbf{H}(\hat{\boldsymbol{\theta}})\right]^{-1} \mathbf{h}(\hat{\boldsymbol{\theta}})
$$

Estatística da Razão de Verossimilhança

$$
\text { - } Q_{L}=-2 \log \lambda=2[L(\hat{\boldsymbol{\theta}})-L(\overline{\boldsymbol{\theta}})]
$$

$$
\text { em que } \lambda=\sup _{\left\{\boldsymbol{\theta}_{\epsilon \Theta: \mathbf{h}}(\boldsymbol{\theta})=0\right\}} L(\boldsymbol{\theta}) / \sup _{\left\{\boldsymbol{\theta}_{\epsilon \Theta}\right\}} L(\boldsymbol{\theta}) \text {. }
$$

Então $Q_{W}$ e $Q_{L}$ têm distribuição assintótica $\chi_{r}^{2}$ sob $H_{0}$ (Russo, 2006).

Continuando na próxima Seção, apresentaremos a estimação restrita sob $H_{0}$ que será utilizada na obtenção das estatísticas dos testes descritos acima.

\subsubsection{Estimação restrita sob $H_{0}$}

Como discutido na Introdução, estamos interessados nos seguintes testes: 
1) $H_{0}: \alpha_{2}=\cdots=\alpha_{p}=0$ e $\beta_{2}=\cdots=\beta_{p}=1$,

2) $H_{0}: \beta_{2}=\cdots=\beta_{p}=1$,

3) $H_{0}: \alpha_{2}=\cdots=\alpha_{p}=0$,

4) $H_{0}: \alpha_{i}=0$ e $\beta_{i}=1, i=2, \cdots, p$,

5) $H_{0}: \alpha_{i}=0, i=2, \cdots, p$,

6) $H_{0}: \beta_{i}=1, i=2, \cdots, p$.

O significado de cada hipótese acima pode ser encontrado na Introdução deste trabalho. Para a hipótese 1 , utilizando a expressão (8) sob $H_{0}$, encontramos o seguinte estimador:

$$
\hat{\mu}_{x_{j}}=\frac{\sum_{i=1}^{p} \sum_{k=1}^{n_{i}} \frac{y_{i j k}}{\sigma_{i j}^{2}}}{\sum_{i=1}^{p} \frac{n_{i}}{\sigma_{i j}^{2}}} .
$$

Para o restante das hipóteses iremos utilizar o algoritmo EM sob cada hipótese $H_{0}$, pois não encontramos uma forma analítica para os estimadores. Consideramos como dados completos $\mathbf{Y}_{c}=\left(\mathbf{Y}_{1 c}, \ldots, \mathbf{Y}_{m c}\right)$, com $\mathbf{Y}_{j c}^{t}=\left(x_{j}, \mathbf{Y}_{j}^{t}\right)^{t}$. Desta forma, o logarítmo da função de verossimilhança completa é dada pela expressão (9) sob $H_{0}$. Assim segue, resumidamente, que:

Para a hipótese:

2) $H_{0}: \beta_{2}=\cdots=\beta_{p}=1$,

Etapa E

$$
\begin{aligned}
& \text { - } \hat{x}_{j}^{(r)}=\frac{\sigma_{x_{j}}^{2}}{\hat{a}_{j 02}^{(r-1)}}\left(\frac{\hat{\mu}_{x_{j}}^{(r-1)}}{\sigma_{x_{j}}^{2}}+\sum_{i=1}^{p} \frac{1}{\sigma_{i j}^{2}} \sum_{k=1}^{n_{i}} y_{i j k}-\sum_{i=2}^{p} \frac{n_{i} \hat{\alpha}_{i}^{(r-1)}}{\sigma_{i j}^{2}}\right), \\
& \text { - }{\widehat{x^{2}}}_{j}^{(r)}=\left(\hat{x}_{j}^{(r)}\right)^{2}+\frac{\sigma_{x_{j}}^{2}}{\hat{a}_{j 02}^{(r-1)}}, \text { com } \hat{a}_{j 02}^{(r-1)}=1+\sigma_{x_{j}}^{2} \sum_{i=1}^{p} \frac{n_{i}}{\sigma_{i j}^{2}} .
\end{aligned}
$$

\section{Etapa M}

$$
\begin{aligned}
\text { - } \hat{\mu}_{x_{j}}^{(r)} & =\hat{x}_{j}^{(r)}, \\
\text { - } \hat{\alpha}_{i}^{(r)} & =\frac{\sum_{j=1}^{m} \sum_{k=1}^{n_{i}} \frac{y_{i j k}}{\sigma_{i j}^{2}}-n_{i} \sum_{j=1}^{m} \frac{\hat{x}_{j}^{(r)}}{\sigma_{i j}^{2}}}{n_{i} \sum_{j=1}^{m} \frac{1}{\sigma_{i j}^{2}}} .
\end{aligned}
$$


3) $H_{0}: \alpha_{2}=\cdots=\alpha_{p}=0$ :

Etapa E

- $\hat{x}_{j}^{(r)}=\frac{\sigma_{x_{j}}^{2}}{\hat{a}_{j 03}^{(r-1)}}\left(\frac{\hat{\mu}_{x_{j}}^{(r-1)}}{\sigma_{x_{j}}^{2}}+\frac{1}{\sigma_{1 j}^{2}} \sum_{k=1}^{n 1} y_{1 j k}+\sum_{i=2}^{p} \frac{\hat{\beta}_{i}^{(r-1)}}{\sigma_{i j}^{2}} \sum_{k=1}^{n i} y_{i j k}\right)$,

- ${\widehat{x^{2}}}_{j}^{(r)}=\left(\hat{x}_{j}^{(r)}\right)^{2}+\frac{\sigma_{x_{j}}^{2}}{\hat{a}_{j 03}^{(r-1)}}$, em que, $\hat{a}_{j 03}^{(r-1)}=1+\sigma_{x_{j}}^{2}\left(\frac{n 1}{\sigma_{1 j}^{2}}+\sum_{i=2}^{p} \frac{n_{i} \hat{\beta}_{i}^{2(r-1)}}{\sigma_{i j}^{2}}\right)$.

Etapa M

- $\hat{\mu}_{x_{j}}^{(r)}=\hat{x}_{j}^{(r)}$,

- $\hat{\beta}_{i}^{(r)}=\frac{\sum_{j=1}^{m} \frac{\hat{x}_{j}^{(r)}}{\sigma_{i j}^{2}} \sum_{k=1}^{n_{i}} y_{i j k}}{n_{i} \sum_{j=1}^{m} \frac{{\widehat{x_{j}^{2}}}^{(r)}}{\sigma_{i j}^{2}}}$.

4) $H_{0}: \alpha_{i}=0$ e $\beta_{i}=1, i=2, \cdots, p$ :

Etapa E

$$
\begin{aligned}
&-\hat{x}_{j}^{(r)}=\frac{\sigma_{x_{j}}^{2}}{\hat{a}_{j 04}^{(r-1)}}\left(\frac{\hat{\mu}_{x_{j}}^{(r-1)}}{\sigma_{x_{j}}^{2}}+\frac{1}{\sigma_{1 j}^{2}} \sum_{k=1}^{n 1} y_{1 j k}+\sum_{k=1}^{n i} \frac{y_{i j k}}{\sigma_{i j}^{2}}+\sum_{l=2, l \neq i}^{p} \frac{\hat{\beta}_{l}^{(r-1)}}{\sigma_{l j}^{2}}\left(\sum_{k=1}^{n l} y_{l j k}\right.\right. \\
&\left.\left.-n_{l} \hat{\alpha}_{l}^{(r-1)}\right)\right) \\
& \text { - }{\widehat{x^{2}}}_{j}^{(r)}=\left(\hat{x}_{j}^{(r)}\right)^{2}+\frac{\sigma_{x_{j}}^{2}}{\hat{a}_{j 04}^{(r-1)}}, \operatorname{com}, \hat{a}_{j 04}^{(r-1)}=1+\sigma_{x_{j}}^{2}\left(\frac{n_{1}}{\sigma_{1 j}^{2}}+\frac{n_{i}}{\sigma_{i j}^{2}}+\sum_{l=2, l \neq i}^{p} \frac{n_{l} \hat{\beta}_{l}^{(r-1) 2}}{\sigma_{l j}^{2}}\right) .
\end{aligned}
$$

Etapa M

- $\hat{\mu}_{x_{j}}^{(r)}=\hat{x}_{j}^{(r)}$,

- $\hat{\alpha}_{l}^{(r)}=\frac{\sum_{j=1}^{m} \sum_{k=1}^{n_{l}} \frac{y_{l j k}}{\sigma_{l j}^{2}}-n_{l} \hat{\beta}_{l}^{(r)} \sum_{j=1}^{m} \frac{\hat{x}_{j}^{(r)}}{\sigma_{l j}^{2}}}{n_{l} \sum_{j=1}^{m} \frac{1}{\sigma_{l j}^{2}}}, l=2, \ldots, p, l \neq i$,

- $\hat{\beta}_{l}^{(r)}=\frac{\frac{1}{n_{l}}\left(\left(\sum_{j=1}^{m} \frac{\hat{x}_{j}^{(r)}}{\sigma_{l j}^{2}} \sum_{k=1}^{n_{l}} y_{l j k}\right)\left(\sum_{j=1}^{m} \frac{1}{\sigma_{l j}^{2}}\right)-\left(\sum_{j=1}^{m} \sum_{k=1}^{n_{l}} \frac{y_{l j k}}{\sigma_{l j}^{2}}\right)\left(\sum_{j=1}^{m} \frac{\hat{x}_{j}^{(r)}}{\sigma_{l j}^{2}}\right)\right)}{\left(\sum_{j=1}^{m} \frac{\widehat{x}_{j}^{(r)}}{\sigma_{l j}^{2}}\right)\left(\sum_{j=1}^{m} \frac{1}{\sigma_{l j}^{2}}\right)-\left(\sum_{j=1}^{m} \frac{\hat{x}_{j}^{(r)}}{\sigma_{l j}^{2}}\right)^{2}}$,

$l=2, \ldots, p, l \neq i$, 
e, portanto, o mesmo estimador de $\alpha_{l}$ e $\beta_{l}$, para $l \neq i$, obtido na estimação irrestrita.

5) $H_{0}: \alpha_{i}=0, i=2, \cdots, p$,

Etapa E

- $\hat{x}_{j}^{(r)}=\frac{\sigma_{x_{j}}^{2}}{\hat{a}_{j 05}^{(r-1)}}\left(\frac{\hat{\mu}_{x_{j}}^{(r-1)}}{\sigma_{x_{j}}^{2}}+\frac{1}{\sigma_{1 j}^{2}} \sum_{k=1}^{n 1} y_{1 j k}+\sum_{l=2}^{p} \frac{\hat{\beta}_{l}^{(r-1)}}{\sigma_{l j}^{2}} \sum_{k=1}^{n_{l}} y_{l j k}-\sum_{l=2, l \neq i}^{p} \frac{\hat{\beta}_{l}^{(r-1)} n_{l} \hat{\alpha}_{l}^{(r-1)}}{\sigma_{l j}^{2}}\right)$,

- ${\widehat{x^{2}}}_{j}^{(r)}=\left(\hat{x}_{j}^{(r)}\right)^{2}+\frac{\sigma_{x_{j}}^{2}}{\hat{a}_{j 05}^{(r-1)}}$, em que, $\hat{a}_{j 05}^{(r-1)}=1+\sigma_{x_{j}}^{2}\left(\frac{n_{1}}{\sigma_{1 j}^{2}}+\sum_{l=2}^{p} \frac{n_{l} \hat{\beta}_{l}^{(r-1) 2}}{\sigma_{l j}^{2}}\right)$.

Etapa M

- $\hat{\mu}_{x_{j}}^{(r)}=\hat{x}_{j}^{(r)}$,

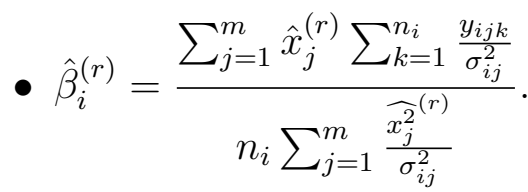

- $\hat{\alpha}_{l}^{(r)}$ e $\hat{\beta}_{l}^{(r)}$ são os mesmos estimadores obtidos na estimação irrestrita para $l=2, \ldots, p, l \neq i$.

6) $H_{0}: \beta_{i}=1, i=2, \cdots, p$ :

Etapa E

- $\hat{x}_{j}^{(r)}=\frac{\sigma_{x_{j}}^{2}}{\hat{a}_{j 06}^{(r-1)}}\left(\frac{\hat{\mu}_{x_{j}}^{(r-1)}}{\sigma_{x j}^{2}}+\frac{1}{\sigma_{1 j}^{2}} \sum_{k=1}^{n 1} y_{1 j k}+\frac{1}{\sigma_{i j}^{2}}\left(\sum_{k=1}^{n_{i}} y_{i j k}-n_{i} \hat{\alpha}_{i}^{(r-1)}\right)\right.$
$\left.\quad+\sum_{l=2, l \neq i}^{p} \frac{\hat{\beta}_{l}^{(r-1)}\left(\sum_{k=1}^{n l} y_{l j k}-n_{l} \hat{\alpha}_{l}^{(r-1)}\right)}{\sigma_{l j}^{2}}\right)$,
- ${\widehat{x^{2}}}^{(r)}=\left(\hat{x}_{j}^{(r)}\right)^{2}+\frac{\sigma_{x j}^{2}}{\hat{a}_{j 06}^{(r-1)}}$, em que, $\hat{a}_{j 06}^{(r-1)}=1+\sigma_{x_{j}}^{2}\left(\frac{n_{1}}{\sigma_{1 j}^{2}}+\frac{n_{i}}{\sigma_{i j}^{2}}+\sum_{l=2, l \neq i}^{p} \frac{n_{l} \hat{\beta}_{l}^{(r-1) 2}}{\sigma_{l j}^{2}}\right)$.

Etapa M

- $\hat{\mu}_{x_{j}}^{(r)}=\hat{x}_{j}^{(r)}$ 
- $\hat{\alpha}_{i}^{(r)}=\frac{\sum_{j=1}^{m} \sum_{k=1}^{n_{i}} \frac{y_{i j k}}{\sigma_{i j}^{2}}+n_{i} \sum_{j=1}^{m} \frac{\hat{x}_{j}^{(r)}}{\sigma_{i j}^{2}}}{n_{i} \sum_{j=1}^{m} \frac{1}{\sigma_{i j}^{2}}}$

- $\hat{\alpha}_{l}^{(r)}$ e $\hat{\beta}_{l}^{(r)}$ são os mesmos estimadores obtidos na estimação irrestrita para $l=2, \ldots, p, l \neq i$. 


\section{Modelo de Calibração t de Student}

Nesta Seção iremos estender o modelo descrito na Introdução, considerando a distribuição t de Student, que tem forma geral simétrica e semelhante à curva da distribuição normal, porém com caudas mais pesadas. O parâmetro $\gamma$ é o número de graus de liberdade. Quanto maior for esse parâmetro, mais próxima da curva da normal ela será.

O modelo $t$ de Student pode produzir estimativas mais robustas às observações atípicas, além de se ajustar mais adequadamente a dados com caudas mais pesadas do que as da distribuição normal. Ao utilizá-lo, podemos minimizar a influência dos outliers presentes nos dados.

Considere agora $z_{i j k}$ como a $k$-ésima medição do verdadeiro valor da potência do motor no $j$-ésimo ponto de rotação medido pelo $i$-ésimo laboratório e $x_{j}$ o verdadeiro valor não observável da potência do motor no $j$-ésimo ponto de rotação, $i=1, \cdots, p$, $j=1, \cdots, m, k=1, \cdots, n_{i}$. Assumindo que $z_{i j k}$ satisfaz a relação linear ultraestrutural com o verdadeiro valor (não observável) $x_{j}$ e denotando por $Z_{i j k}$ o valor observado (sujeito a erro de medição) da $k$-ésima medição da potência do motor no $j$-ésimo ponto de rotação obtido pelo $i$-ésimo laboratório, temos:

$$
\begin{gathered}
z_{i j k}=\alpha_{i}+\beta_{i} x_{j}, \\
Z_{i j k}=z_{i j k}+e_{i j k},
\end{gathered}
$$

$i=1, \cdots, p, j=1, \cdots, m$ e $k=1, \cdots, n_{i}$. Considerando o laboratório 1 como o laboratório de referência, obtemos:

$$
\begin{gathered}
Z_{1 j k}=x_{j}+e_{1 j k}, \\
Z_{i j k}=\alpha_{i}+\beta_{i} x_{j}+e_{i j k},
\end{gathered}
$$


$i=2, \ldots, p, j=1, \ldots, m$ e $k=1, \ldots, n_{i}$.

Seja $\mathbf{Z}_{j}=\left(\mathbf{Z}_{1 j}^{t}, \ldots, \mathbf{Z}_{p j}^{t}\right)^{t}, \operatorname{com} \mathbf{Z}_{i j}=\left(Z_{i j 1}, \ldots, Z_{i j n_{i}}\right)^{t}, j=1, \ldots, m$ e $i=1, \ldots, p$. Assumindo que $\mathbf{Z}_{j} \sim t_{n}\left(\boldsymbol{\mu}_{j}, \boldsymbol{\Lambda}_{j}, \gamma\right)$, em que $t_{r}(\boldsymbol{\mu}, \boldsymbol{\Lambda}, \gamma)$ denota uma distribuição t de Student $r$-variada com vetor de locação $\boldsymbol{\mu}$, matriz escala $\boldsymbol{\Lambda}$ e $\gamma$ graus de liberdade e $\boldsymbol{\Lambda}_{j}=\frac{\gamma-2}{\gamma} \boldsymbol{\Sigma}_{j}, \operatorname{com} \boldsymbol{\mu}_{j}, \boldsymbol{\Sigma}_{j}$ e n como definidos em (5), temos que:

$$
\operatorname{Var}\left(\mathbf{Z}_{j}\right)=\frac{\gamma}{\gamma-2} \boldsymbol{\Lambda}_{j}=\boldsymbol{\Sigma}_{j}
$$

Desta forma,

$$
\begin{gathered}
f_{\mathbf{Z}_{j}}\left(\mathbf{z}_{j}\right)=\frac{\Gamma\left(\frac{\gamma+n}{2}\right)}{\Gamma\left(\frac{\gamma}{2}\right) \gamma^{\frac{n}{2}} \pi^{\frac{n}{2}}}\left|\boldsymbol{\Lambda}_{j}\right|^{-\frac{1}{2}}\left[1+\frac{1}{\gamma}\left(\mathbf{Z}_{j}-\boldsymbol{\mu}_{j}\right)^{t} \boldsymbol{\Lambda}_{j}^{-1}\left(\mathbf{Z}_{j}-\boldsymbol{\mu}_{j}\right)\right]^{-\left(\frac{\gamma+n}{2}\right)}= \\
\frac{\Gamma\left(\frac{\gamma+n}{2}\right)}{\Gamma\left(\frac{\gamma}{2}\right) \gamma^{\frac{n}{2}} \pi^{\frac{n}{2}}}\left(\frac{\gamma-2}{\gamma}\right)^{-\frac{n}{2}}\left|\boldsymbol{\Sigma}_{j}\right|^{-\frac{1}{2}}\left[\frac{\gamma-2+\left(\mathbf{Z}_{j}-\boldsymbol{\mu}_{j}\right)^{t} \boldsymbol{\Sigma}_{j}^{-1}\left(\mathbf{Z}_{j}-\boldsymbol{\mu}_{j}\right)}{\gamma-2}\right]^{-\left(\frac{\gamma+n}{2}\right)} \\
\mathrm{e}, \\
\log f_{\mathbf{Z}_{j}}\left(\mathbf{z}_{j}\right)=c t e-\frac{1}{2} \log \left|\boldsymbol{\Sigma}_{j}\right|-\left(\frac{\gamma+n}{2}\right) \log \left(\gamma-2+Q_{j}\right), \text { em que } \\
Q_{j}=\left(\mathbf{Z}_{j}-\boldsymbol{\mu}_{j}\right)^{t} \boldsymbol{\Sigma}_{j}^{-1}\left(\mathbf{Z}_{j}-\boldsymbol{\mu}_{j}\right) .
\end{gathered}
$$

Assim,

$L(\boldsymbol{\theta})=\log f_{\mathbf{Z}}(\mathbf{z})=\sum_{j=1}^{m} f_{\mathbf{Z}_{j}}\left(\mathbf{z}_{j}\right)=c t e-\frac{1}{2} \sum_{j=1}^{m} \log \left|\boldsymbol{\Sigma}_{j}\right|-\left(\frac{\gamma+n}{2}\right) \sum_{j=1}^{m} \log \left(\gamma-2+Q_{j}\right)$,

onde, $\mathbf{Z}=\left(\mathbf{Z}_{1}^{t}, \ldots, \mathbf{Z}_{m}^{t}\right)^{t}$ e $\boldsymbol{\Sigma}_{j}$ como visto em (5).

Na próxima subseção, apresentamos a função score e a matriz de informação observada.

\subsection{Função escore e matriz de informação observada}

A função escore para o modelo descrito acima é dada por: $U(\boldsymbol{\theta})=\frac{\partial L(\boldsymbol{\theta})}{\partial \boldsymbol{\theta}}=$ $\left(U\left(\boldsymbol{\theta}_{1}\right)^{t}, U\left(\boldsymbol{\theta}_{2}\right)^{t}, U\left(\boldsymbol{\theta}_{3}\right)^{t}\right)^{t}$ onde, $\boldsymbol{\theta}=\left(\boldsymbol{\theta}_{1}^{t}, \boldsymbol{\theta}_{2}^{t}, \boldsymbol{\theta}_{3}^{t}\right)^{t} \operatorname{com}, \boldsymbol{\theta}_{1}=\left(\mu_{x 1}, \ldots, \mu_{x m}\right)^{t}, \boldsymbol{\theta}_{2}=$ $\left(\alpha_{2}, \ldots, \alpha_{p}\right)^{t}, \boldsymbol{\theta}_{3}=\left(\beta_{2}, \ldots, \beta_{p}\right)^{t} \mathrm{e}$ 


$$
\begin{aligned}
U\left(\boldsymbol{\theta}_{1}\right) & =-\frac{1}{2}(\gamma+n) \frac{1}{\gamma-2+Q_{j}} \frac{\partial Q_{j}}{\partial \boldsymbol{\theta}_{1}}, \\
U\left(\boldsymbol{\theta}_{2}\right) & =-\frac{1}{2}(\gamma+n) \sum_{j=1}^{m} \frac{1}{\gamma-2+Q_{j}} \frac{\partial Q_{j}}{\partial \boldsymbol{\theta}_{2}}, \\
U\left(\boldsymbol{\theta}_{3}\right) & =-\frac{1}{2} \sum_{j=1}^{m} \frac{\partial \log \left|\boldsymbol{\Sigma}_{j}\right|}{\partial \boldsymbol{\theta}_{3}}-\frac{1}{2}(\gamma+n) \sum_{j=1}^{m} \frac{1}{\gamma-2+Q_{j}} \frac{\partial Q_{j}}{\partial \boldsymbol{\theta}_{3}} .
\end{aligned}
$$

As derivadas parciais de $\log \left|\boldsymbol{\Sigma}_{j}\right|$ e $Q_{j}$ são as mesmas vistas anteriormente no Capítulo 2 (partes I e II) substituindo $y_{1 j k}$ e $y_{i j k}$ por $z_{1 j k}$ e $z_{i j k}$ respectivamente, com $i=$ $2, \ldots p, j=1, \ldots, m$ e $k=1, \ldots, n_{i}$.

A matriz de informação observada é dada por $I(\boldsymbol{\theta})=-\frac{\partial^{2} L(\boldsymbol{\theta})}{\partial \boldsymbol{\theta} \partial \boldsymbol{\theta}^{t}}$, em que, $\boldsymbol{\theta}=$ $\left(\boldsymbol{\theta}_{1}^{t}, \boldsymbol{\theta}_{2}^{t}, \boldsymbol{\theta}_{3}^{t}\right)^{t}$ com, $\boldsymbol{\theta}_{1}^{t}=\left(\mu_{x 1}, \ldots, \mu_{x m}\right)^{t}, \boldsymbol{\theta}_{2}^{t}=\left(\alpha_{2}, \ldots, \alpha_{p}\right)^{t}, \boldsymbol{\theta}_{3}^{t}=\left(\beta_{2}, \ldots, \beta_{p}\right)^{t}$ e portanto,

$$
I(\boldsymbol{\theta})=\left[\begin{array}{ccc}
{ }^{I_{\boldsymbol{\theta}_{1}} \boldsymbol{\theta}_{1}} & { }^{I_{\boldsymbol{\theta}_{1}} \boldsymbol{\theta}_{2}} & { }^{I} \boldsymbol{\theta}_{1} \boldsymbol{\theta}_{3} \\
I_{\boldsymbol{\theta}_{2} \boldsymbol{\theta}_{1}} & { }^{I_{\boldsymbol{\theta}_{2}} \boldsymbol{\theta}_{2}} & { }^{I_{\boldsymbol{\theta}_{2}} \boldsymbol{\theta}_{3}} \\
{ }^{I_{\boldsymbol{\theta}_{3} \boldsymbol{\theta}_{1}}} & { }^{I_{\boldsymbol{\theta}_{3}} \boldsymbol{\theta}_{2}} & { }^{I_{\boldsymbol{\theta}_{3}} \boldsymbol{\theta}_{3}}
\end{array}\right],
$$

onde,

$$
\begin{aligned}
& I_{\boldsymbol{\theta}_{1} \boldsymbol{\theta}_{1}}=\frac{(\gamma+n)}{2}\left(-\frac{1}{\left(\gamma-2+Q_{j}\right)^{2}} \frac{\partial Q_{j}}{\partial \boldsymbol{\theta}_{1}} \frac{\partial Q_{j}}{\partial \boldsymbol{\theta}_{1}{ }^{t}}+\frac{1}{\left(\gamma-2+Q_{j}\right)} \frac{\partial^{2} Q_{j}}{\partial \boldsymbol{\theta}_{1} \partial \boldsymbol{\theta}_{1}{ }^{t}}\right),
\end{aligned}
$$

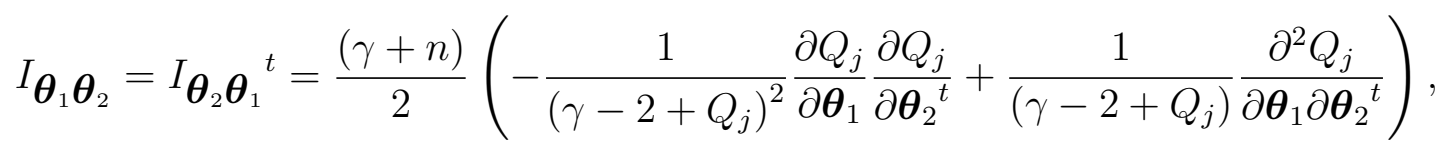

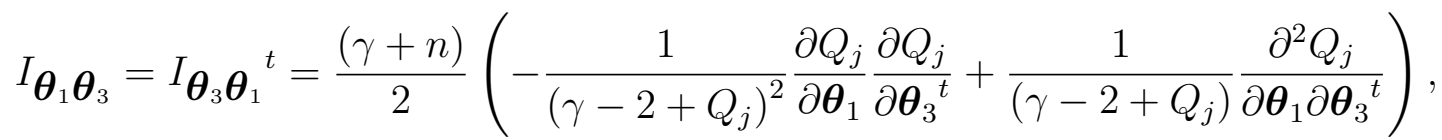

$$
\begin{aligned}
& I_{\boldsymbol{\theta}_{2} \boldsymbol{\theta}_{2}}=\frac{(\gamma+n)}{2}\left(\sum_{j=1}^{m}-\frac{1}{\left(\gamma-2+Q_{j}\right)^{2}} \frac{\partial Q_{j}}{\partial \boldsymbol{\theta}_{2}} \frac{\partial Q_{j}}{\partial \boldsymbol{\theta}_{2}{ }^{t}}+\sum_{j=1}^{m} \frac{1}{\left(\gamma-2+Q_{j}\right)} \frac{\partial^{2} Q_{j}}{\partial \boldsymbol{\theta}_{2} \partial \boldsymbol{\theta}_{2}{ }^{t}}\right) \text {, } \\
& I_{\boldsymbol{\theta}_{2} \boldsymbol{\theta}_{3}}=I_{\boldsymbol{\theta}_{3} \boldsymbol{\theta}_{2}}=\frac{(\gamma+n)}{2}\left(\sum_{j=1}^{m}-\frac{1}{\left(\gamma-2+Q_{j}\right)^{2}} \frac{\partial Q_{j}}{\partial \boldsymbol{\theta}_{2}} \frac{\partial Q_{j}}{\partial \boldsymbol{\theta}_{3}{ }^{t}}+\sum_{j=1}^{m} \frac{1}{\left(\gamma-2+Q_{j}\right)} \frac{\partial^{2} Q_{j}}{\partial \boldsymbol{\theta}_{2} \partial \boldsymbol{\theta}_{3}{ }^{t}}\right) \text {, } \\
& { }^{I_{\boldsymbol{\theta}_{3}} \boldsymbol{\theta}_{3}}=\frac{1}{2} \sum_{j=1}^{m} \frac{\partial^{2} \log \left|\boldsymbol{\Sigma}_{j}\right|}{\partial \boldsymbol{\theta}_{3} \partial \boldsymbol{\theta}_{3}{ }^{t}}-\frac{(\gamma+n)}{2} \sum_{j=1}^{m} \frac{1}{\left(\gamma-2+Q_{j}\right)^{2}} \frac{\partial Q_{j}}{\partial \boldsymbol{\theta}_{3}} \frac{\partial Q_{j}}{\partial \boldsymbol{\theta}_{3}{ }^{t}}+
\end{aligned}
$$


$+\frac{(\gamma+n)}{2} \sum_{j=1}^{m} \frac{1}{\left(\gamma-2+Q_{j}\right)} \frac{\partial^{2} Q_{j}}{\partial \boldsymbol{\theta}_{3} \partial \boldsymbol{\theta}_{3}{ }^{t}}$,

Novamente, as derivadas parciais de segunda ordem de $\log \left|\boldsymbol{\Sigma}_{j}\right|$ e $Q_{j}$ são as mesmas vistas anteriormente no Capítulo 2, substituindo $y_{1 j k}$ e $y_{i j k}$ por $z_{1 j k}$ e $z_{i j k}$ respectivamente, $\operatorname{com} i=2, \ldots, p, j=1, \ldots, m, k=1, \ldots, n_{i}$.

Apresentamos a seguir, um método para a estimação dos parâmetros do modelo e descrevemos os testes de hipóteses de interesse.

\subsection{Estimação dos parâmetros e testes de hipóteses}

Estamos interessados em obter as estimativas para os parâmetros do novo modelo, para tanto utilizaremos o algoritmo EM. Seja $\mathbf{T}_{j}=\left(x_{j}, \mathbf{Z}_{j}^{t}\right)^{t} \sim t_{n+1}\left(\boldsymbol{\mu}_{t j}, \boldsymbol{\Lambda}_{t j}, \gamma\right)$, em que $\boldsymbol{\mu}_{t j}=\left(\mu_{x j}, \boldsymbol{\mu}_{j}^{t}\right)^{t}, \boldsymbol{\Lambda}_{t j}=\left(\frac{\gamma-2}{\gamma}\right) \boldsymbol{\Sigma}_{t j}$

$$
\boldsymbol{\Sigma}_{t j}=\left[\begin{array}{cc}
\sigma_{x j}^{2} & \sigma_{x j}^{2} \boldsymbol{\beta}^{t} \\
\sigma_{x j}^{2} \boldsymbol{\beta} & \boldsymbol{\Sigma} j
\end{array}\right]
$$

$\boldsymbol{\mu}_{j}$ e $\boldsymbol{\Sigma}_{j}$ como em (4) e (5).

Considere $W_{j} \sim \frac{\chi_{(\gamma)}^{2}}{\gamma}, \gamma>0, j=1, \ldots, m$, então $\mathbf{T}_{j} \mid W_{j}=w_{j} \sim N_{n+1}\left(\boldsymbol{\mu}_{t j}, w_{j}^{-1} \boldsymbol{\Lambda}_{t j}\right)$.

Desta forma, o logarítmo da função de verossimilhança completa é dada por:

$L_{c}(\boldsymbol{\theta})=\log \prod_{j=1}^{m} f\left(\mathbf{t}_{j}, w_{j}\right)=\log \prod_{j=1}^{m} f\left(\mathbf{t}_{j} \mid w_{j}\right) f\left(w_{j}\right)=$

$\sum_{j=1}^{m} \log \left[(2 \pi)^{-\frac{(n+1)}{2}}\left|w_{j}^{-1} \boldsymbol{\Lambda}_{t j}\right|^{-\frac{1}{2}} \exp \left\{-\frac{1}{2}\left(\mathbf{T}_{j}-\boldsymbol{\mu}_{t j}\right)^{t} w_{j} \boldsymbol{\Lambda}_{t j}^{-1}\left(\mathbf{T}_{j}-\boldsymbol{\mu}_{t j}\right)\right\} f\left(w_{j}\right)\right]$,

em que, $f\left(\mathbf{t}_{j}, w_{j}\right)$ denota a função densidade de probabilidade (fdp) conjunta de $\mathbf{T}_{j}$ e $W_{j}, f\left(\mathbf{t}_{j} / w_{j}\right)$ denota a densidade condicionada de $t_{j}$ dado $w_{j}$ e $f\left(w_{j}\right)$ denota a fdp de $W_{j}$.

Portanto,

$L_{c}(\boldsymbol{\theta})=c t e-\frac{1}{2} \sum_{j=1}^{m} \log \left|\boldsymbol{\Sigma}_{t j}\right|-\frac{1}{2}\left(\frac{\gamma-2}{\gamma}\right)^{-1} \sum_{j=1}^{m} w_{j}\left(\mathbf{T}_{j}-\boldsymbol{\mu}_{t j}\right)^{t} \boldsymbol{\Sigma}_{t j}^{-1}\left(\mathbf{T}_{j}-\boldsymbol{\mu}_{t j}\right)$,

Logo, podemos reescrever: 


$$
\begin{aligned}
L_{c}(\boldsymbol{\theta}) & =\text { cte }-\frac{1}{2}\left(\frac{\gamma}{\gamma-2}\right)\left[\sum_{j=1}^{m} w_{j} \frac{\left(x_{j}-\mu_{x j}\right)^{2}}{\sigma_{x j}^{2}}+\sum_{j=1}^{m} \sum_{k=1}^{n 1} w_{j} \frac{\left(z_{1 j k}-x_{j}\right)^{2}}{\sigma_{1 j}^{2}}\right. \\
& \left.+\sum_{j=1}^{m} \sum_{i=2}^{p} \sum_{k=1}^{n i} w_{j} \frac{\left(z_{i j k}-\alpha_{i}-\beta_{i} x_{j}\right)^{2}}{\sigma_{i j}^{2}}\right]
\end{aligned}
$$

ou ainda,

$$
\begin{gathered}
L_{c}(\boldsymbol{\theta})=c t e-\frac{1}{2}\left(\frac{\gamma}{\gamma-2}\right)\left[\sum_{j=1}^{m} w_{j} x_{j}^{2}\left(\frac{1}{\sigma_{x j}^{2}}+\frac{n_{1}}{\sigma_{1 j}^{2}}+\sum_{i=2}^{p} \frac{n_{i} \beta_{i}^{2}}{\sigma_{i j}^{2}}\right)\right. \\
-2 \sum_{j=1}^{m} w_{j} x_{j}\left(\frac{\mu_{x j}}{\sigma_{x j}^{2}}+\sum_{k=1}^{n_{1}} \frac{z_{1 j k}}{\sigma_{1 j}^{2}}+\sum_{i=2}^{p} \sum_{k=1}^{n_{i}} \frac{z_{i j k} \beta_{i}-\alpha_{i} \beta_{i}}{\sigma_{i j}^{2}}\right) \\
\left.+\sum_{j=1}^{m} w_{j} \frac{\mu_{x j}^{2}}{\sigma_{x j}^{2}}+\sum_{j=1}^{m} \sum_{k=1}^{n_{1}} w_{j} \frac{z_{1 j k}^{2}}{\sigma_{1 j}^{2}}+\sum_{j=1}^{m} \sum_{i=2}^{p} \sum_{k=1}^{n_{i}} w_{j} \frac{\left(z_{i j k}-\alpha_{i}\right)^{2}}{\sigma_{i j}^{2}}\right]
\end{gathered}
$$

\subsubsection{Algoritmo EM}

\section{Etapa E}

Seguindo os mesmos passos vistos na Seção 2.3.2, segue que para o novo modelo, temos:

$$
\begin{aligned}
& \hat{w}_{j}^{(r)}=E\left[w_{j} \mid \mathbf{Z}_{j}, \hat{\boldsymbol{\theta}}^{(r-1)}\right]=\frac{(\gamma-2)(\gamma+n)}{\gamma\left(\gamma-2+\hat{Q}_{j}^{(r-1)}\right)}, \\
& \hat{x}_{j}^{(r)}=E\left[x_{j} \mid \mathbf{Z}_{j}, \hat{\boldsymbol{\theta}}^{(r-1)}\right]=\frac{\sigma_{x_{j}}^{2}}{\hat{a}_{j}^{(r-1)}}\left(\frac{\hat{\mu}_{x_{j}}^{(r-1)}}{\sigma_{x_{j}}^{2}}+\frac{1}{\sigma_{1 j}^{2}} \sum_{k=1}^{n 1} z_{1 j k}+\sum_{i=2}^{p} \frac{\hat{\beta}_{i}^{(r-1)}}{\sigma_{i j}^{2}}\left(\sum_{k=1}^{n i} z_{i j k}-n_{i} \hat{\alpha}_{i}^{(r-1)}\right)\right),
\end{aligned}
$$

em que

$$
\begin{aligned}
& \hat{a}_{j}^{(r-1)}=1+\sigma_{x_{j}}^{2}\left(\frac{n_{1}}{\sigma_{1 j}^{2}}+\sum_{i=2}^{p} \frac{n_{i} \hat{\beta}_{i}^{2(r-1)}}{\sigma_{i j}^{2}}\right) \\
& {\widehat{x^{2}}}^{(r)}=E\left[x_{j}^{2} \mid \mathbf{Z}_{j}, \hat{\boldsymbol{\theta}}^{(r-1)}\right]=\left(\hat{x}_{j}^{(r)}\right)^{2}+\frac{\sigma_{x_{j}}^{2}}{\hat{a}_{j}^{(r-1)}}\left(\frac{\gamma-2+\hat{Q}_{j}^{(r-1)}}{\gamma+n-2}\right)
\end{aligned}
$$


Desta forma:

$$
\begin{gathered}
E\left(L_{c}(\boldsymbol{\theta}) \mid \mathbf{Z}, \hat{\boldsymbol{\theta}}^{(r-1)}\right)=c t e-\frac{1}{2}\left(\frac{\gamma}{\gamma-2}\right)\left[\sum_{j=1}^{m} \hat{w}_{j}^{(r)} \widehat{x}_{j}^{2}(r)\left(\frac{1}{\sigma_{x_{j}}^{2}}+\frac{n_{1}}{\sigma_{1 j}^{2}}+\sum_{i=2}^{p} \frac{n_{i} \hat{\beta}_{i}^{2(r-1)}}{\sigma_{i j}^{2}}\right)\right. \\
+\sum_{j=1}^{m} \hat{w}_{j}^{(r)} \hat{x}_{j}^{(r)}\left(-2 \frac{\hat{\mu}_{x_{j}}^{(r-1)}}{\sigma_{x_{j}}^{2}}-2 \sum_{k=1}^{n_{1}} \frac{z_{1 j k}}{\sigma_{1 j}^{2}}-2 \sum_{i=2}^{p} \sum_{k=1}^{n_{i}} \frac{z_{i j k} \hat{\beta}_{i}^{(r-1)}-\hat{\alpha}_{i}^{(r-1)} \hat{\beta}_{i}^{(r-1)}}{\sigma_{i j}^{2}}\right) \\
\left.+\sum_{j=1}^{m} \hat{w}_{j}^{(r)} \frac{\hat{\mu}_{x_{j}}^{2(r-1)}}{\sigma_{x_{j}}^{2}}+\sum_{j=1}^{m} \sum_{k=1}^{n_{1}} \hat{w}_{j}^{(r)} \frac{z_{1 j k}^{2}}{\sigma_{1 j}^{2}}+\sum_{j=1}^{m} \sum_{i=2}^{p} \sum_{k=1}^{n_{i}} \hat{w}_{j}^{(r)} \frac{\left(z_{i j k}-\hat{\alpha}_{i}^{(r-1)}\right)^{2}}{\sigma_{i j}^{2}}\right]
\end{gathered}
$$

\section{Etapa M}

$$
\begin{aligned}
& \hat{\mu}_{x j}^{(r)}=\hat{x}_{j}^{(r)}, j=1, \ldots, m, \\
& \hat{\alpha}_{i}^{(r)}=\frac{\sum_{j=1}^{m} \sum_{k=1}^{n_{i}} \hat{w}_{j}^{(r)} \frac{z_{i j k}}{\sigma_{i j}^{2}}-n_{i} \hat{\beta}_{i}^{(r)} \sum_{j=1}^{m} \hat{w}_{j}^{(r)} \frac{\hat{x}_{j}^{(r)}}{\sigma_{i j}^{2}}}{n_{i} \sum_{j=1}^{m} \frac{\hat{w}_{j}^{(r)}}{\sigma_{i j}^{2}}, i=2, \ldots, p,} \\
& \hat{\beta}_{i}^{(r)}=\frac{\frac{1}{n_{i}}\left(\left(\sum_{j=1}^{m} \frac{\hat{x}_{j}^{(r)} \hat{w}_{j}^{(r)}}{\sigma_{i j}^{2}} \sum_{k=1}^{n_{i}} z_{i j k}\right)\left(\sum_{j=1}^{m} \frac{\hat{w}_{j}^{(r)}}{\sigma_{i j}^{2}}\right)-\left(\sum_{j=1}^{m} \sum_{k=1}^{n_{i}} \hat{w}_{j}^{(r)} \frac{z_{i j k}}{\sigma_{i j}^{2}}\right)\left(\sum_{j=1}^{m} \hat{w}_{j}^{(r)} \frac{\hat{x}_{j}^{(r)}}{\sigma_{i j}^{2}}\right)\right)}{\left(\sum_{j=1}^{m} \frac{\widehat{x}_{j}^{(r)} \hat{w}_{j}^{(r)}}{\sigma_{i j}^{2}}\right)\left(\sum_{j=1}^{m} \frac{\hat{w}_{j}^{(r)}}{\sigma_{i j}^{2}}\right)-\left(\sum_{j=1}^{m} \frac{\hat{x}_{j}^{(r)} \hat{w}_{j}^{(r)}}{\sigma_{i j}^{2}}\right)^{2}} \\
& i=2, \ldots, p .
\end{aligned}
$$

\subsubsection{Testes de hipóteses assintóticos}

Nesta Seção vamos utilizar os testes apresentados na Seção 2.3.3.

\subsubsection{Estimação restrita sob $H_{0}$}

Como discutido na introdução, estamos interessados nos testes descritos na página 15, agora para o novo modelo.

Para a hipótese 1, utilizando a expressão (10) sob $H_{0}$, encontramos o seguinte estimador:

$$
\hat{\mu}_{x j}=\frac{\sum_{i=1}^{p} \sum_{k=1}^{n_{i}} \frac{z_{i j k}}{\sigma_{i j}^{2}}}{\sum_{i=1}^{p} \frac{n_{i}}{\sigma_{i j}^{2}}} .
$$


Para o restante das hipóteses iremos utilizar o algorítmo EM sob cada hipótese $H_{0}$, pois, novamente, não encontramos uma forma analítica para os estimadores. $\mathrm{O}$ logarítmo da função de verossimilhança completa é dada pela expressão (11) sob $H_{0}$. Assim, para a hipótese:

2) $H_{0}: \beta_{2}=\cdots=\beta_{p}=1$,

Etapa E

$$
\begin{aligned}
& \text { - } \hat{w}_{j}^{(r)}=\frac{(\gamma-2)(\gamma+n)}{\gamma\left(\gamma-2+\hat{Q}_{j 02}^{(r-1)}\right)}, \\
& \text { - } \hat{x}_{j}^{(r)}=\frac{\sigma_{x j}^{2}}{\hat{a}_{j 02}^{(r-1)}}\left(\frac{\hat{\mu}_{x_{j}}^{(r-1)}}{\sigma_{x_{j}}^{2}}+\sum_{i=1}^{p} \frac{1}{\sigma_{i j}^{2}} \sum_{k=1}^{n_{i}} z_{i j k}-\sum_{i=2}^{p} \frac{n_{i} \hat{\alpha}_{i}^{(r-1)}}{\sigma_{i j}^{2}}\right), \\
& \text { - }{\widehat{x^{2}}}_{j}^{(r)}=\left(\hat{x}_{j}^{(r)}\right)^{2}+\frac{\sigma_{x j}^{2}}{\hat{a}_{j 02}^{(r-1)}}\left(\frac{\gamma-2+\hat{Q}_{j 02}^{(r-1)}}{\gamma+n-2}\right)
\end{aligned}
$$

$\operatorname{com} \hat{a}_{j 02}^{(r-1)}=1+\sigma_{x_{j}}^{2} \sum_{i=1}^{p} \frac{n_{i}}{\sigma_{i j}^{2}}$ e $\hat{Q}_{j 02}^{(r-1)}=\left(\mathbf{Z}_{j}-\boldsymbol{\mu}_{j 02}\right)^{t} \boldsymbol{\Sigma}_{j 02}^{-1}\left(\mathbf{Z}_{j}-\boldsymbol{\mu}_{j 02}\right)$, onde $\boldsymbol{\mu}_{j 02}=$ $\boldsymbol{\alpha}+\mu_{x_{j}} \mathbf{1}_{n}$ e $\boldsymbol{\Sigma}_{j 02}=D\left(\boldsymbol{\sigma}_{j}^{2}\right)+\sigma_{x_{j}}^{2} \mathbf{1}_{n} \mathbf{1}_{n}^{t}$, com $\boldsymbol{\alpha}$ como em (5) e $\boldsymbol{\sigma}_{j}^{2}$ como em (6).

\section{Etapa M}

- $\hat{\mu}_{x_{j}}^{(r)}=\hat{x}_{j}^{(r)}$,

- $\hat{\alpha}_{i}^{(r)}=\frac{\sum_{j=1}^{m} \sum_{k=1}^{n_{i}} \frac{\hat{w}_{j}^{(r)} z_{i j k}}{\sigma_{i j}^{2}}-n_{i} \sum_{j=1}^{m} \frac{\hat{w}_{j}^{(r)} \hat{x}_{j}^{(r)}}{\sigma_{i j}^{2}}}{n_{i} \sum_{j=1}^{m} \frac{\hat{w}_{j}^{(r)}}{\sigma_{i j}^{2}}}$.

3) $H_{0}: \alpha_{2}=\cdots=\alpha_{p}=0$ :

Etapa E

$$
\begin{aligned}
\text { - } \hat{w}_{j}^{(r)} & =\frac{(\gamma-2)(\gamma+n)}{\gamma\left(\gamma-2+\hat{Q}_{j 03}^{(r-1)}\right)}, \\
\text { - } \hat{x}_{j}^{(r)} & =\frac{\sigma_{x_{j}}^{2}}{\hat{a}_{j}^{(r-1)}}\left(\frac{\hat{\mu}_{x_{j}}^{(r-1)}}{\sigma_{x_{j}}^{2}}+\frac{1}{\sigma_{1 j}^{2}} \sum_{k=1}^{n 1} z_{1 j k}+\sum_{i=2}^{p} \frac{\hat{\beta}_{i}^{(r-1)}}{\sigma_{i j}^{2}} \sum_{k=1}^{n i} z_{i j k}\right),
\end{aligned}
$$




$$
\text { - }{\widehat{x^{2}}}_{j}^{(r)}=\left(\hat{x}_{j}^{(r)}\right)^{2}+\frac{\sigma_{x_{j}}^{2}}{\hat{a}_{j}^{(r-1)}}\left(\frac{\gamma-2+\hat{Q}_{j 03}^{(r-1)}}{\gamma+n-2}\right)
$$

em que, $\hat{a}_{j}$ é o mesmo da Seção 3.2.1, Etapa E e $\hat{Q}_{j 03}^{(r-1)}=\left(\mathbf{Z}_{j}-\boldsymbol{\mu}_{j 03}\right)^{t} \boldsymbol{\Sigma}_{j}^{-1}\left(\mathbf{Z}_{j}-\boldsymbol{\mu}_{j 03}\right)$, em que $\boldsymbol{\mu}_{j 03}=\mu_{x_{j}} \boldsymbol{\beta}$, com $\boldsymbol{\beta}$ como em (5).

Etapa M

$$
\begin{aligned}
\text { - } \hat{\mu}_{x_{j}}^{(r)}=\hat{x}_{j}^{(r)}, \\
\text { - } \hat{\beta}_{i}^{(r)}=\frac{\sum_{j=1}^{m} \frac{\hat{w}_{j}^{(r)} \hat{x}_{j}^{(r)}}{\sigma_{i j}^{2}} \sum_{k=1}^{n_{i}} z_{i j k}}{n_{i} \sum_{j=1}^{m} \frac{\hat{w}_{j}^{(r)}{\widehat{x_{j}^{2}}}^{(r)}}{\sigma_{i j}^{2}}} .
\end{aligned}
$$

4) $H_{0}: \alpha_{i}=0$ e $\beta_{i}=1, \quad i=2, \cdots, p$ :

Etapa E

$$
\begin{aligned}
& \text { - } \hat{w}_{j}^{(r)}=\frac{(\gamma-2)(\gamma+n)}{\gamma\left(\gamma-2+\hat{Q}_{j 04}^{(r-1)}\right)} \\
& \text { • } \hat{x}_{j}^{(r)}=\frac{\sigma_{x_{j}}^{2}}{\hat{a}_{j 04}^{(r-1)}}\left(\frac{\hat{\mu}_{x_{j}}^{(r-1)}}{\sigma_{x_{j}}^{2}}+\frac{1}{\sigma_{1 j}^{2}} \sum_{k=1}^{n 1} z_{1 j k}+\sum_{k=1}^{n i} \frac{z_{i j k}}{\sigma_{i j}^{2}}+\sum_{l=2, l \neq i}^{p} \frac{\hat{\beta}_{l}^{(r-1)}}{\sigma_{l j}^{2}}\left(\sum_{k=1}^{n l} z_{l j k}\right.\right. \\
& \left.\left.-n_{l} \hat{\alpha}_{l}^{(r-1)}\right)\right) \text {, } \\
& \text { - }{\widehat{x^{2}}}_{j}^{(r)}=\left(\hat{x}_{j}^{(r)}\right)^{2}+\frac{\sigma_{x j}^{2}}{\hat{a}_{j 04}^{(r-1)}}\left(\frac{\gamma-2+\hat{Q}_{j 04}^{(r-1)}}{\gamma+n-2}\right),
\end{aligned}
$$

em que,

$\hat{a}_{j 04}^{(r-1)}=1+\sigma_{x_{j}}^{2}\left(\frac{n_{1}}{\sigma_{1 j}^{2}}+\frac{n_{i}}{\sigma_{i j}^{2}}+\sum_{l=2, l \neq i}^{p} \frac{n_{l} \hat{\beta}_{l}^{(r-1) 2}}{\sigma_{l j}^{2}}\right)$ e $\hat{Q}_{j 04}^{(r-1)}=\left(\mathbf{Z}_{j}-\boldsymbol{\mu}_{j 04}\right)^{t} \boldsymbol{\Sigma}_{j 04}^{-1}\left(\mathbf{Z}_{j}-\boldsymbol{\mu}_{j 04}\right)$,

$\operatorname{com} \boldsymbol{\mu}_{j 04}=\left(\boldsymbol{\mu}_{1 j}^{t}, \ldots, \boldsymbol{\mu}_{(i-1) j}^{t}, \boldsymbol{\mu}_{i j_{04}}^{t}, \boldsymbol{\mu}_{(i+1) j}^{t}, \ldots, \boldsymbol{\mu}_{p j}^{t}\right)^{t}$ e $\boldsymbol{\Sigma}_{j 04}=D\left(\boldsymbol{\sigma}_{j}^{2}\right)+\sigma_{x_{j}}^{2} \boldsymbol{\beta}_{04} \boldsymbol{\beta}_{04}^{t}, \mathrm{e}$ ainda, $\boldsymbol{\mu}_{i j_{04}}=\mu_{x_{j}} \mathbf{1}_{n i}$ e $\boldsymbol{\beta}_{04}=\left(\mathbf{1}_{n 1}^{t}, \beta_{2} \mathbf{1}_{n_{2}}^{t}, \ldots, \beta_{(i-1)} \mathbf{1}_{n_{(i-1)}}^{t}, \mathbf{1}_{n_{i}}^{t}, \beta_{(i+1)} \mathbf{1}_{n_{(i+1)}}^{t}, \ldots, \beta_{p} \mathbf{1}_{n_{p}}^{t}\right)^{t}$.

Etapa M 
- $\hat{\mu}_{x_{j}}^{(r)}=\hat{x}_{j}^{(r)}$,

$$
\begin{aligned}
\text { - } \hat{\alpha}_{l}^{(r)}= & \frac{\sum_{j=1}^{m} \sum_{k=1}^{n_{l}} \frac{\hat{w}_{j}^{(r)} z_{l j k}}{\sigma_{l j}^{2}}-n_{l} \hat{\beta}_{l}^{(r)} \sum_{j=1}^{m} \frac{\hat{w}_{j}^{(r)} \hat{x}_{j}^{(r)}}{\sigma_{l j}^{2}}}{n_{l} \sum_{j=1}^{m} \frac{\hat{w}_{j}^{(r)}}{\sigma_{l j}^{2}}}, l=2, \ldots, p, l \neq i, \\
\text { - } \hat{\beta}_{l}^{(r)}= & \frac{\frac{1}{n_{l}}\left(\sum_{j=1}^{m} \frac{\hat{w}_{j}^{(r)} \hat{x}_{j}^{(r)}}{\sigma_{l j}^{2}} \sum_{k=1}^{n_{l}} z_{l j k}\right)\left(\sum_{j=1}^{m} \frac{\hat{w}_{j}^{(r)}}{\sigma_{l j}^{2}}\right)}{\left(\sum_{j=1}^{m} \frac{\hat{w}_{j}^{(r)} \widehat{x}_{j}^{(r)}}{\sigma_{l j}^{2}}\right)\left(\sum_{j=1}^{m} \frac{\hat{w}_{j}^{(r)}}{\sigma_{l j}^{2}}\right)-\left(\sum_{j=1}^{m} \frac{\hat{w}_{j}^{(r)} \hat{x}_{j}^{(r)}}{\sigma_{l j}^{2}}\right)^{2}}- \\
- & \frac{\frac{1}{n_{l}}\left(\sum_{j=1}^{m} \sum_{k=1}^{n_{l}} \frac{\hat{w}_{j}^{(r)} z_{l j k}}{\sigma_{l j}^{2}}\right)\left(\sum_{j=1}^{m} \frac{\hat{w}_{j}^{(r)} \hat{x}_{j}^{(r)}}{\sigma_{l j}^{2}}\right)}{\left(\sum_{j=1}^{m} \frac{\hat{w}_{j}^{(r)} \widehat{x}_{j}^{(r)}}{\sigma_{l j}^{2}}\right)\left(\sum_{j=1}^{m} \frac{\hat{w}_{j}^{(r)}}{\sigma_{l j}^{2}}\right)-\left(\sum_{j=1}^{m} \frac{\hat{w}_{j}^{(r)} \hat{x}_{j}^{(r)}}{\sigma_{l j}^{2}}\right)^{2}}, l=2, \ldots, p, l \neq i,
\end{aligned}
$$

e, portanto, o mesmo estimador de $\alpha_{l}$ e $\beta_{l}$, para $l \neq i$, obtido na estimação irrestrita.

5) $H_{0}: \alpha_{i}=0, i=2, \cdots, p$,

\section{Etapa E}

$$
\begin{aligned}
\text { - } \hat{w}_{j}^{(r)} & =\frac{(\gamma-2)(\gamma+n)}{\gamma\left(\gamma-2+\hat{Q}_{j 05}^{(r-1)}\right)}, \\
\text { - } \hat{x}_{j}^{(r)} & =\frac{\sigma_{x_{j}}^{2}}{\hat{a}_{j 05}^{(r-1)}}\left(\frac{\hat{\mu}_{x_{j}}^{(r-1)}}{\sigma_{x_{j}}^{2}}+\frac{1}{\sigma_{1 j}^{2}} \sum_{k=1}^{n 1} z_{1 j k}+\sum_{l=2}^{p} \frac{\hat{\beta}_{l}^{(r-1)}}{\sigma_{l j}^{2}} \sum_{k=1}^{n_{l}} z_{l j k}-\sum_{l=2, l \neq i}^{p} \frac{\hat{\beta}_{l}^{(r-1)} n_{l} \hat{\alpha}_{l}^{(r-1)}}{\sigma_{l j}^{2}}\right), \\
\text { - }{\widehat{x^{2}}}^{(r)} & =\left(\hat{x}_{j}^{(r)}\right)^{2}+\frac{\sigma_{x_{j}}^{2}}{\hat{a}_{j}^{(r-1)}}\left(\frac{\gamma-2+\hat{Q}_{j 05}^{(r-1)}}{\gamma+n-2}\right),
\end{aligned}
$$

onde, $\hat{a}_{j}^{(r-1)}$ é o mesmo visto na Seção 3.2.1, etapa E e $\hat{Q}_{j 05}^{(r-1)}=\left(\mathbf{Z}_{j}-\boldsymbol{\mu}_{j 05}\right)^{t} \boldsymbol{\Sigma}_{j}^{-1}\left(\mathbf{Z}_{j}-\boldsymbol{\mu}_{j 05}\right)$, $\operatorname{com} \boldsymbol{\mu}_{j 05}=\left(\boldsymbol{\mu}_{1 j}^{t}, \ldots, \boldsymbol{\mu}_{(i-1) j}^{t}, \boldsymbol{\mu}_{i j_{05}}^{t}, \boldsymbol{\mu}_{(i+1) j}^{t}, \ldots, \boldsymbol{\mu}_{p j}^{t}\right)^{t}$, e ainda, $\boldsymbol{\mu}_{i j_{05}}=\mu_{x_{j}} \beta_{i} \mathbf{1}_{n_{i}}$.

Etapa M

- $\hat{\mu}_{x_{j}}^{(r)}=\hat{x}_{j}^{(r)}$,

$$
\text { - } \hat{\beta}_{i}^{(r)}=\frac{\sum_{j=1}^{m} \hat{w}_{j}^{(r)} \hat{x}_{j}^{(r)} \sum_{k=1}^{n_{i}} \frac{z_{i j k}}{\sigma_{i j}^{2}}}{n_{i} \sum_{j=1}^{m} \frac{\hat{w}_{j}^{(r)} \widehat{x}_{j}^{(r)}}{\sigma_{i j}^{2}}}
$$


- $\hat{\alpha}_{l}^{(r)}$ e $\hat{\beta}_{l}^{(r)}$ são os mesmos estimadores obtidos na estimação irrestrita para

$$
l=2, \ldots, p, l \neq i \text {. }
$$

6) $H_{0}: \beta_{i}=1, i=2, \cdots, p$ :

Etapa E

$$
\begin{aligned}
& \text { - } \hat{w}_{j}^{(r)}=\frac{(\gamma-2)(\gamma+n)}{\gamma\left(\gamma-2+\hat{Q}_{j 06}^{(r-1)}\right)}, \\
& \text { - } \hat{x}_{j}^{(r)}=\frac{\sigma_{x_{j}}^{2}}{\hat{a}_{j 06}^{(r-1)}}\left(\frac{\hat{\mu}_{x_{j}}^{(r-1)}}{\sigma_{x_{j}}^{2}}+\frac{1}{\sigma_{1 j}^{2}} \sum_{k=1}^{n 1} z_{1 j k}+\frac{1}{\sigma_{i j}^{2}}\left(\sum_{k=1}^{n_{i}} z_{i j k}-n_{i} \hat{\alpha}_{i}^{(r-1)}\right)\right. \\
& \left.+\sum_{l=2, l \neq i}^{p} \frac{\hat{\beta}_{l}^{(r-1)}\left(\sum_{k=1}^{n l} z_{l j k}-n_{l} \hat{\alpha}_{l}^{(r-1)}\right)}{\sigma_{l j}^{2}}\right), \\
& \text { - }{\widehat{x^{2}}}_{j}^{(r)}=\left(\hat{x}_{j}^{(r)}\right)^{2}+\frac{\sigma_{x j}^{2}}{\hat{a}_{j 06}^{(r-1)}}\left(\frac{\gamma-2+\hat{Q}_{j 06}^{(r-1)}}{\gamma+n-2}\right) \text {, }
\end{aligned}
$$

onde, $\hat{a}_{j 06}^{(r-1)}=1+\sigma_{x_{j}}^{2}\left(\frac{n_{1}}{\sigma_{1 j}^{2}}+\frac{n_{i}}{\sigma_{i j}^{2}}+\sum_{l=2, l \neq i}^{p} \frac{n_{l} \hat{\beta}_{l}^{(r-1) 2}}{\sigma_{l j}^{2}}\right)$ e $\hat{Q}_{j 06}^{(r-1)}=\left(\mathbf{Z}_{j}-\boldsymbol{\mu}_{j 06}\right)^{t} \boldsymbol{\Sigma}_{j 06}^{-1}\left(\mathbf{Z}_{j}-\boldsymbol{\mu}_{j 06}\right)$, $\operatorname{com} \boldsymbol{\mu}_{j 06}=\left(\boldsymbol{\mu}_{1 j}^{t}, \ldots, \boldsymbol{\mu}_{(i-1) j}^{t}, \boldsymbol{\mu}_{i j_{06}}^{t}, \boldsymbol{\mu}_{(i+1) j}^{t}, \ldots, \boldsymbol{\mu}_{p j}^{t}\right)^{t}$ e $\boldsymbol{\Sigma}_{j 06}=D\left(\boldsymbol{\sigma}_{j}^{2}\right)+\sigma_{x_{j}}^{2} \boldsymbol{\beta}_{06} \boldsymbol{\beta}_{06}^{t}$, e ainda, $\boldsymbol{\mu}_{i j_{06}}=\left(\alpha_{i}+\mu_{x_{j}}\right) \mathbf{1}_{n_{i}}^{t}$ e $\boldsymbol{\beta}_{06}=\left(\mathbf{1}_{n_{1}}^{t}, \beta_{2} \mathbf{1}_{n_{2}}^{t}, \ldots, \beta_{(i-1)} \mathbf{1}_{n_{(i-1)}}^{t}, \mathbf{1}_{n_{i}}^{t}, \beta_{(i+1)} \mathbf{1}_{n_{(i+1)}}^{t}, \ldots, \beta_{p} \mathbf{1}_{n_{p}}^{t}\right)^{t}$.

Etapa M

- $\hat{\mu}_{x_{j}}^{(r)}=\hat{x}_{j}^{(r)}$,

- $\hat{\alpha}_{i}^{(r)}=\frac{\sum_{j=1}^{m} \sum_{k=1}^{n_{i}} \frac{\hat{w}_{j}^{(r)} z_{i j k}}{\sigma_{i j}^{2}}-n_{i} \sum_{j=1}^{m} \frac{\hat{w}_{j}^{(r)} \hat{x}_{j}^{(r)}}{\sigma_{i j}^{2}}}{n_{i} \sum_{j=1}^{m} \frac{\hat{w}_{j}^{(r)}}{\sigma_{i j}^{2}}}$,

- $\hat{\alpha}_{l}^{(r)}$ e $\hat{\beta}_{l}^{(r)}$ são os mesmos estimadores obtidos na estimação irrestrita para $l=2, \ldots, p, l \neq i$. 


\section{Aplicação}

Considerando os dados descritos na motivação deste trabalho, obtivemos as estimativas dos parâmetros do modelo considerando a distribuição normal e a distribuição t de Student e testamos as hipóteses de interesse.

\subsection{Resultados utilizando a distribuição Normal}

Considerando o conjunto de dados das medições da potência do motor realizadas pelos diversos laboratórios participantes em 10 pontos de rotação (Apêndice D), obtivemos as EMV dos parâmetros do modelo considerando o algoritmo EM descrito na seção 2.3.1. Na Tabela 1 apresentamos os valores iniciais usados no algorítmo, o parâmetro $\mu_{x_{j}}$ foi calculado da seguinte maneira: $\mu_{x_{j}}=\sum_{i=1}^{p} \frac{y_{i j k}}{n}$, onde $n=\sum_{i=1}^{p} n_{i}$ e os parâmetros $\alpha_{i}$ e $\beta_{i}$ foram assumidos 0 e 1, respectivamente, e na Tabela 2 as estimativas de máxima verossimilhança dos parâmetros.

Tabela 1: Valores iniciais usados no algorítmo EM- Caso Normal.

\begin{tabular}{rrr}
\hline$\mu_{x_{j}}$ & $\alpha_{i}$ & $\beta_{i}$ \\
\hline 19,56 & 0 & 1 \\
26,9 & 0 & 1 \\
34,94 & 0 & 1 \\
45,08 & 0 & 1 \\
53,66 & 0 & 1 \\
62,18 & 0 & 1 \\
69,72 & & \\
74,84 & & \\
78,44 & & \\
79,04 & & \\
\hline
\end{tabular}

O algoritmo EM foi implementado utilizando o programa R. O critério de convergência utilizado foi calculado da seguinte forma:

$\left(\mu_{x_{j}}^{(r)}-\mu_{x_{j}}^{(r-1)}\right)^{2}+\left(\alpha_{i}^{(r)}-\alpha_{i}^{(r-1)}\right)^{2}+\left(\beta_{i}^{(r)}-\beta_{i}^{(r-1)}\right)^{2}<\epsilon$, onde $\epsilon=10^{-9}$ A seguir, apresentamos os resultados da estimação restrita nas Tabelas 3, 4 e 5, e os resultados 
Tabela 2: Estimativa de máxima verossimilhança (EMV) dos parâmetros usando o algoritmo EM. Desvios Padrões estimados (DPE) entre parênteses.

\begin{tabular}{rrr}
\hline$\mu_{x_{j}}$ & $\alpha_{i}$ & $\beta_{i}$ \\
\hline $19,717(0,121)$ & $0,224(0,164)$ & $0,992(0,004)$ \\
$27,318(0,151)$ & $-0,188(0,189)$ & $0,970(0,004)$ \\
$35,562(0,189)$ & $0,418(0,132)$ & $0,987(0,003)$ \\
$45,680(0,242)$ & $0,074(0,447)$ & $0,989(0,008)$ \\
$54,540(0,289)$ & $0,243(0,133)$ & $0,978(0,003)$ \\
$63,552(0,337)$ & & \\
$71,320(0,379)$ & & \\
$76,692(0,409)$ & & \\
$79,831(0,429)$ & & \\
$79,790(0,433)$ & & \\
\hline
\end{tabular}

dos testes assintóticos na Tabela 6 .

Tabela 3: Estimativa restrita de máxima verossimilhança de $\mu_{x_{j}}$.

\begin{tabular}{lcccccccccc}
\hline & \multicolumn{10}{c}{ Parâmetros } \\
Hipóteses & $\mu_{x_{1}}$ & $\mu_{x_{2}}$ & $\mu_{x_{3}}$ & $\mu_{x_{4}}$ & $\mu_{x_{5}}$ & $\mu_{x_{6}}$ & $\mu_{x_{7}}$ & $\mu_{x_{8}}$ & $\mu_{x_{9}}$ & $\mu_{x_{10}}$ \\
\hline 1 & 19,676 & 27,163 & 35,258 & 45,200 & 53,908 & 62,748 & 70,374 & 75,669 & 78,763 & 78,720 \\
2 & 19,947 & 27,440 & 35,565 & 45,527 & 54,246 & 63,109 & 70,745 & 76,037 & 79,130 & 79,088 \\
3 & 19,855 & 27,413 & 35,608 & 45,670 & 54,484 & 63,451 & 71,181 & 76,527 & 79,649 & 79,603 \\
$4(\mathrm{i}=2)$ & 19,744 & 27,315 & 35,526 & 45,603 & 54,428 & 63,405 & 71,144 & 76,495 & 79,622 & 79,583 \\
$4(\mathrm{i}=3)$ & 19,509 & 26,959 & 35,035 & 44,945 & 53,625 & 62,445 & 70,051 & 75,320 & 78,393 & 78,345 \\
$4(\mathrm{i}=4)$ & 19,834 & 27,363 & 35,527 & 45,546 & 54,320 & 63,245 & 70,936 & 76,256 & 79,362 & 79,315 \\
$4(\mathrm{i}=5)$ & 19,725 & 27,300 & 35,516 & 45,597 & 54,424 & 63,401 & 71,137 & 76,487 & 79,612 & 79,569 \\
$4(\mathrm{i}=6)$ & 19,582 & 27,064 & 35,173 & 45,127 & 53,842 & 62,704 & 70,343 & 75,633 & 78,728 & 78,685 \\
$5(\mathrm{i}=2)$ & 19,778 & 27,360 & 35,585 & 45,678 & 54,516 & 63,507 & 71,256 & 76,616 & 79,747 & 79,707 \\
$5(\mathrm{i}=3)$ & 19,681 & 27,291 & 35,547 & 45,679 & 54,553 & 63,580 & 71,360 & 76,741 & 79,886 & 79,844 \\
$5(\mathrm{i}=4)$ & 19,888 & 27,440 & 35,627 & 45,675 & 54,474 & 63,425 & 71,138 & 76,474 & 79,590 & 79,545 \\
$5(\mathrm{i}=5)$ & 19,720 & 27,320 & 35,564 & 45,680 & 54,539 & 63,550 & 71,317 & 76,689 & 79,827 & 79,785 \\
$5(\mathrm{i}=6)$ & 19,815 & 27,386 & 35,599 & 45,677 & 54,503 & 63,479 & 71,216 & 76,568 & 79,695 & 79,654 \\
$6(\mathrm{i}=2)$ & 19,778 & 27,348 & 35,560 & 45,636 & 54,461 & 63,437 & 71,176 & 76,526 & 79,653 & 79,614 \\
$6(\mathrm{i}=3)$ & 19,918 & 27,428 & 35,567 & 45,547 & 54,283 & 63,164 & 70,817 & 76,113 & 79,205 & 79,163 \\
$6(\mathrm{i}=4)$ & 19,864 & 27,394 & 35,558 & 45,577 & 54,351 & 63,276 & 70,967 & 76,288 & 79,394 & 79,347 \\
$6(\mathrm{i}=5)$ & 19,738 & 27,330 & 35,564 & 45,668 & 54,515 & 63,513 & 71,267 & 76,629 & 79,762 & 79,719 \\
$6(\mathrm{i}=6)$ & 19,961 & 27,440 & 35,555 & 45,511 & 54,229 & 63,094 & 70,735 & 76,024 & 79,117 & 79,075 \\
\hline
\end{tabular}


Tabela 4: Estimativa restrita de máxima verossimilhança de $\alpha_{i}$.

\begin{tabular}{lccccc}
\hline \multicolumn{7}{c}{ Parâmetros } \\
Hipóteses & $\alpha_{2}$ & $\alpha_{3}$ & $\alpha_{4}$ & $\alpha_{5}$ & $\alpha_{6}$ \\
\hline 2 & $-0,079$ & $-1,361$ & $-0,036$ & $-0,220$ & $-0,534$ \\
$4(\mathrm{i}=2)$ & 0,000 & $-0,289$ & 0,315 & $-0,028$ & 0,140 \\
$4(\mathrm{i}=3)$ & 0,023 & 0,000 & 0,217 & $-0,130$ & 0,044 \\
$4(\mathrm{i}=4)$ & $-0,086$ & $-0,492$ & 0,000 & $-0,238$ & $-0,063$ \\
$4(\mathrm{i}=5)$ & 0,142 & $-0,271$ & 0,335 & 0,000 & 0,161 \\
$4(\mathrm{i}=6)$ & 0,036 & $-0,372$ & 0,231 & $-0,114$ & 0,000 \\
$5(\mathrm{i}=2)$ & 0,000 & $-0,293$ & 0,311 & $-0,033$ & 0,137 \\
$5(\mathrm{i}=3)$ & 0,290 & 0,000 & 0,484 & 0,142 & 0,308 \\
$5(\mathrm{i}=4)$ & $-0,082$ & $-0,489$ & 0,000 & $-0,234$ & $-0,060$ \\
$5(\mathrm{i}=5)$ & 0,219 & $-0,193$ & 0,413 & 0,000 & 0,238 \\
$5(\mathrm{i}=6)$ & 0,050 & $-0,359$ & 0,245 & $-0,100$ & 0,000 \\
$6(\mathrm{i}=2)$ & $-0,068$ & $-0,323$ & 0,280 & $-0,063$ & 0,106 \\
$6(\mathrm{i}=3)$ & $-0,254$ & $-1,377$ & $-0,060$ & $-0,419$ & $-0,230$ \\
$6(\mathrm{i}=4)$ & $-0,116$ & $-0,522$ & $-0,042$ & $-0,268$ & $-0,093$ \\
$6(\mathrm{i}=5)$ & 0,174 & $-0,238$ & 0,368 & $-0,493$ & 0,193 \\
$6(\mathrm{i}=6)$ & $-0,339$ & 0,739 & $-0,143$ & $-0,488$ & $-0,533$ \\
\hline
\end{tabular}

Tabela 5: Estimativa restrita de máxima verossimilhança de $\beta_{i}$.

\begin{tabular}{lccccc}
\hline \multicolumn{7}{c}{ Parâmetros } \\
Hipóteses & $\beta_{2}$ & $\beta_{3}$ & $\beta_{4}$ & $\beta_{5}$ & $\beta_{6}$ \\
\hline 3 & 0,997 & 0,967 & 0,996 & 0,992 & 0,983 \\
$4(\mathrm{i}=2)$ & 1,000 & 0,974 & 0,991 & 0,993 & 0,982 \\
$4(\mathrm{i}=3)$ & 1,012 & 1,000 & 1,008 & 1,010 & 0,998 \\
$4(\mathrm{i}=4)$ & 1,001 & 0,980 & 1,000 & 0,999 & 0,988 \\
$4(\mathrm{i}=5)$ & 0,995 & 0,974 & 0,991 & 1,000 & 0,982 \\
$4(\mathrm{i}=6)$ & 1,008 & 0,986 & 1,003 & 1,005 & 1,000 \\
$5(\mathrm{i}=2)$ & 0,997 & 0,972 & 0,989 & 0,991 & 0,980 \\
$5(\mathrm{i}=3)$ & 0,990 & 0,966 & 0,985 & 0,988 & 0,977 \\
$5(\mathrm{i}=4)$ & 0,998 & 0,977 & 0,996 & 0,996 & 0,985 \\
$5(\mathrm{i}=5)$ & 0,992 & 0,970 & 0,987 & 0,990 & 0,978 \\
$5(\mathrm{i}=6)$ & 0,995 & 0,974 & 0,991 & 0,993 & 0,983 \\
$6(\mathrm{i}=2)$ & 1,000 & 0,974 & 0,991 & 0,993 & 0,982 \\
$6(\mathrm{i}=3)$ & 1,005 & 1,000 & 1,001 & 1,003 & 0,991 \\
$6(\mathrm{i}=4)$ & 1,001 & 0,980 & 1,000 & 0,999 & 0,988 \\
$6(\mathrm{i}=5)$ & 0,993 & 0,971 & 0,988 & 1,000 & 0,979 \\
$6(\mathrm{i}=6)$ & 1,008 & 0,986 & 1,003 & 1,005 & 1,000 \\
\hline
\end{tabular}


Tabela 6: Resultados das estatísticas Wald e Razão de Verossimilhança(RV).

\begin{tabular}{rrrrr}
\hline & Wald & P-Valor & RV & P-Valor \\
Hipóteses & & & & \\
\hline 1 & 490,481 & 0,000 & 482,356 & 0,000 \\
2 & 73,497 & 0,000 & 71,250 & 0,000 \\
3 & 19,109 & 0,002 & 18,922 & 0,002 \\
$4(\mathrm{i}=2)$ & 4,897 & 0,086 & 4,861 & 0,088 \\
$4(\mathrm{i}=3)$ & 304,615 & 0,000 & 295,889 & 0,000 \\
$4(\mathrm{i}=4)$ & 15,982 & 0,000 & 15,677 & 0,000 \\
$4(\mathrm{i}=5)$ & 9,954 & 0,007 & 9,903 & 0,007 \\
$4(\mathrm{i}=6)$ & 116,230 & 0,000 & 112,559 & 0,000 \\
$5(\mathrm{i}=2)$ & 1,878 & 0,171 & 1,869 & 0,172 \\
$5(\mathrm{i}=3)$ & 0,990 & 0,320 & 0,992 & 0,319 \\
$5(\mathrm{i}=4)$ & 9,993 & 0,002 & 9,860 & 0,002 \\
$5(\mathrm{i}=5)$ & 0,028 & 0,868 & 0,028 & 0,868 \\
$5(\mathrm{i}=6)$ & 3,333 & 0,068 & 3,309 & 0,069 \\
$6(\mathrm{i}=2)$ & 4,099 & 0,043 & 4,068 & 0,044 \\
$6(\mathrm{i}=3)$ & 50,510 & 0,000 & 49,152 & 0,000 \\
$6(\mathrm{i}=4)$ & 15,524 & 0,000 & 15,229 & 0,000 \\
$6(\mathrm{i}=5)$ & 1,907 & 0,167 & 1,902 & 0,168 \\
$6(\mathrm{i}=6)$ & 43,705 & 0,000 & 42,332 & 0,000 \\
\hline & & & &
\end{tabular}

De acordo com a Tabela 6, segue que:

- para a hipótese $1\left(H_{0}: \alpha_{2}=\cdots=\alpha_{p}=0\right.$ e $\left.\beta_{2}=\cdots=\beta_{p}=1\right)$, concluímos que rejeita-se a hipótese nula para os dois testes (Wald e RV), ou seja, o grupo de motores não é consistente;

- para a hipótese $2\left(H_{0}: \beta_{2}=\cdots=\beta_{p}=1\right)$, concluímos que pelo menos um dos laboratórios está medindo com vício multiplicativo;

- para a hipótese $3\left(H_{0}: \alpha_{2}=\cdots=\alpha_{p}=0\right)$, concluímos que pelo menos um dos laboratórios está medindo com vício aditivo;

- para a hipótese $4\left(H_{0}: \alpha_{i}=0\right.$ e $\left.\beta_{i}=1, i=2, \cdots, p\right)$, para o laboratório $i=2$ adotando os níveis de significância 1 e 5\%, não rejeita-se a hipótese nula para os dois testes, porém a 10\% rejeita-se a hipótese nula, ou seja, o laboratório 
em questão não mede sem vícios; para os demais laboratórios, concluímos que rejeita-se a hipótese nula para os dois testes, isto é, estes laboratórios não medem sem vícios.

- para a hipótese $5\left(H_{0}: \alpha_{i}=0, i=2, \cdots, p\right)$, para os laboratórios $i=2,3,5$, adotando os níveis de significância 1,5 e 10\% concluímos que não rejeita-se a hipótese nula para os dois testes, ou seja, estes laboratórios medem sem vícios aditivos; para o laboratório $i=4$, concluímos que este laboratório não mede sem vícios aditivos; para o laboratório $i=6$, adotando os níveis de significância $1 \mathrm{e}$ $5 \%$, não rejeita-se a hipótese nula para os dois testes, porém a $10 \%$ a hipótese nula é rejeitada para os dois testes, portanto, mede com vícios aditivos;

- para a hipótese $6\left(H_{0}: \beta_{i}=1, i=2, \cdots, p\right)$, para o laboratório $i=2$, adotando os níveis de significância 5 e 10\%, rejeita-se a hipótese nula para os dois testes, ou seja, este laboratório não mede sem vícios multiplicativos; a 1\% não rejeita-se a hipótese nula para os dois testes; para os laboratórios $i=3,4,6$, concluímos que rejeita-se a hipótese nula nos níveis de significância anteriormente considerados, isto é, tais laboratórios não medem sem vícios multiplicativos; para o laboratório $i=5$, concluímos que não rejeita-se a hipótese nula para os dois testes nos níveis 1,5 e $10 \%$.

\subsection{Resultados utilizando a distribuição t de Student}

Nesta Seção, assim como apresentamos os resultados do caso Normal, apresentaremos os resultados do caso t de Student. Foi usado o valor de 3.4 para o grau de liberdade, pois conforme vemos pelas figuras abaixo, para este valor foram obtidos os menores valores de AIC e BIC, que são critérios de seleção de modelos, onde o objetivo é encontrar a menor ordem possível que consiga adequar satisfatoriamente os dados do modelo (Sobral \& Barreto (2011)). 

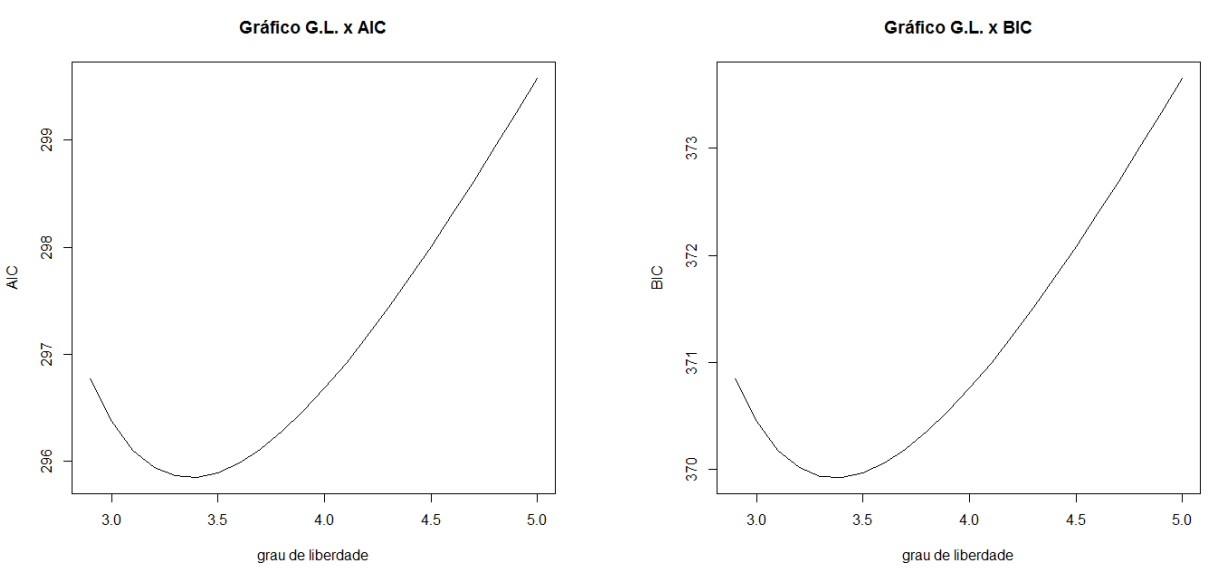

Os valores iniciais são os mesmos do caso Normal. Apresentamos os valores das estimativas de máxima verossimilhança usando o algoritmo EM na Tabela 7. Em seguida, apresentamos os valores das estimativas restritas nas Tabelas 8, 9 e 10, e na sequência, os resultados dos testes assintóticos na Tabela 11. 
Tabela 7: EMV usando o algoritmo EM (t de Student). DPE em parênteses.

\begin{tabular}{rrr}
\hline$\mu_{x_{j}}$ & $\alpha_{i}$ & $\beta_{i}$ \\
\hline $19,717(0,080)$ & $0,199(0,118)$ & $0,994(0,003)$ \\
$27,299(0,075)$ & $0,050(0,123)$ & $0,963(0,003)$ \\
$35,525(0,084)$ & $0,237(0,099)$ & $0,995(0,003)$ \\
$45,622(0,157)$ & $0,042(0,271)$ & $0,991(0,006)$ \\
$54,466(0,171)$ & $0,265(0,093)$ & $0,997(0,003)$ \\
$63,469(0,206)$ & & \\
$71,225(0,292)$ & & \\
$76,586(0,269)$ & & \\
$79,715(0,455)$ & & \\
$79,665(0,800)$ & & \\
\hline
\end{tabular}

Tabela 8: Estimativa restrita de máxima verossimilhança de $\mu_{x_{j}}$.

\begin{tabular}{lcccccccccc}
\hline & \multicolumn{10}{c}{ Parâmetros } \\
Hipóteses & $\mu_{x_{1}}$ & $\mu_{x_{2}}$ & $\mu_{x_{3}}$ & $\mu_{x_{4}}$ & $\mu_{x_{5}}$ & $\mu_{x_{6}}$ & $\mu_{x_{7}}$ & $\mu_{x_{8}}$ & $\mu_{x_{9}}$ & $\mu_{x_{10}}$ \\
\hline 1 & 19,676 & 27,163 & 35,258 & 45,200 & 53,908 & 62,748 & 70,374 & 75,669 & 78,763 & 78,720 \\
2 & 19,913 & 27,404 & 35,532 & 45,496 & 54,217 & 63,082 & 70,719 & 76,010 & 79,103 & 79,061 \\
3 & 19,812 & 27,353 & 35,535 & 45,581 & 54,380 & 63,337 & 71,056 & 76,391 & 79,506 & 79,455 \\
$4(\mathrm{i}=2)$ & 19,756 & 27,315 & 35,516 & 45,583 & 54,401 & 63,377 & 71,112 & 76,457 & 79,577 & 79,529 \\
$4(\mathrm{i}=3)$ & 19,548 & 26,982 & 35,040 & 44,929 & 53,592 & 62,396 & 69,987 & 75,244 & 78,308 & 78,258 \\
$4(\mathrm{i}=4)$ & 19,808 & 27,362 & 35,555 & 45,613 & 54,423 & 63,391 & 71,117 & 76,458 & 79,573 & 79,521 \\
$4(\mathrm{i}=5)$ & 19,726 & 27,287 & 35,489 & 45,557 & 54,374 & 63,348 & 71,079 & 76,422 & 79,540 & 79,488 \\
$4(\mathrm{i}=6)$ & 19,594 & 27,061 & 35,155 & 45,095 & 53,799 & 62,656 & 70,288 & 75,571 & 78,660 & 78,611 \\
$5(\mathrm{i}=2)$ & 19,766 & 27,328 & 35,533 & 45,605 & 54,427 & 63,407 & 71,145 & 76,493 & 79,614 & 79,565 \\
$5(\mathrm{i}=3)$ & 19,726 & 27,304 & 35,527 & 45,620 & 54,460 & 63,458 & 71,211 & 76,569 & 79,696 & 79,646 \\
$5(\mathrm{i}=4)$ & 19,800 & 27,350 & 35,540 & 45,593 & 54,399 & 63,363 & 71,086 & 76,424 & 79,538 & 79,486 \\
$5(\mathrm{i}=5)$ & 19,718 & 27,300 & 35,525 & 45,622 & 54,465 & 63,466 & 71,222 & 76,583 & 79,711 & 79,661 \\
$5(\mathrm{i}=6)$ & 19,808 & 27,352 & 35,538 & 45,586 & 54,387 & 63,345 & 71,063 & 76,399 & 79,514 & 79,464 \\
$6(\mathrm{i}=2)$ & 19,757 & 27,315 & 35,517 & 45,584 & 54,402 & 63,378 & 71,113 & 76,458 & 79,578 & 79,529 \\
$6(\mathrm{i}=3)$ & 19,934 & 27,412 & 35,519 & 45,460 & 54,163 & 63,014 & 70,639 & 75,913 & 78,990 & 78,942 \\
$6(\mathrm{i}=4)$ & 19,768 & 27,321 & 35,515 & 45,573 & 54,382 & 63,350 & 71,077 & 76,417 & 79,533 & 79,480 \\
$6(\mathrm{i}=5)$ & 19,734 & 27,307 & 35,523 & 45,607 & 54,439 & 63,428 & 71,172 & 76,524 & 79,647 & 79,596 \\
$6(\mathrm{i}=6)$ & 19,918 & 27,382 & 35,483 & 45,426 & 54,134 & 62,995 & 70,631 & 75,913 & 79,000 & 78,952 \\
\hline
\end{tabular}


Tabela 9: Estimativa restrita de máxima verossimilhança de $\alpha_{i}$.

\begin{tabular}{lccccc}
\hline \multicolumn{7}{c}{ Parâmetros } \\
Hipóteses & $\alpha_{2}$ & $\alpha_{3}$ & $\alpha_{4}$ & $\alpha_{5}$ & $\alpha_{6}$ \\
\hline 2 & 0,020 & $-1,400$ & 0,040 & $-0,209$ & $-0,543$ \\
$4(\mathrm{i}=2)$ & 0,000 & $-0,029$ & 0,137 & $-0,051$ & 0,166 \\
$4(\mathrm{i}=3)$ & $-0,084$ & 0,000 & 0,072 & $-0,217$ & $-0,005$ \\
$4(\mathrm{i}=4)$ & 0,022 & $-0,098$ & 0,000 & $-0,121$ & 0,106 \\
$4(\mathrm{i}=5)$ & 0,122 & $-0,008$ & 0,170 & 0,000 & 0,191 \\
$4(\mathrm{i}=6)$ & $-0,034$ & $-0,124$ & 0,073 & $-0,152$ & 0,000 \\
$5(\mathrm{i}=2)$ & 0,000 & $-0,032$ & 0,135 & $-0,053$ & 0,169 \\
$5(\mathrm{i}=3)$ & 0,181 & 0,000 & 0,219 & 0,024 & 0,248 \\
$5(\mathrm{i}=4)$ & 0,022 & $-0,094$ & 0,000 & $-0,119$ & 0,105 \\
$5(\mathrm{i}=5)$ & 0,195 & 0,046 & 0,233 & 0,000 & 0,262 \\
$5(\mathrm{i}=6)$ & 0,005 & $-0,119$ & 0,052 & $-0,139$ & 0,000 \\
$6(\mathrm{i}=2)$ & $-0,001$ & $-0,029$ & 0,137 & $-0,052$ & 0,166 \\
$6(\mathrm{i}=3)$ & $-0,316$ & $-1,349$ & $-0,246$ & $-0,509$ & $-0,307$ \\
$6(\mathrm{i}=4)$ & 0,065 & $-0,054$ & 0,056 & $-0,078$ & 0,143 \\
$6(\mathrm{i}=5)$ & 0,151 & 0,009 & 0,192 & $-0,349$ & 0,220 \\
$6(\mathrm{i}=6)$ & $-0,323$ & $-0,407$ & $-0,240$ & $-0,453$ & $-0,479$ \\
\hline
\end{tabular}

Tabela 10: Estimativa restrita de máxima verossimilhança de $\beta_{i}$.

\begin{tabular}{lccccc}
\hline \multicolumn{7}{c}{ Parâmetros } \\
Hipóteses & $\beta_{2}$ & $\beta_{3}$ & $\beta_{4}$ & $\beta_{5}$ & $\beta_{6}$ \\
\hline 3 & 0,999 & 0,965 & 1,001 & 0,993 & 0,984 \\
$4(\mathrm{i}=2)$ & 1,000 & 0,966 & 0,998 & 0,994 & 0,980 \\
$4(\mathrm{i}=3)$ & 1,015 & 1,000 & 1,012 & 1,012 & 0,999 \\
$4(\mathrm{i}=4)$ & 0,998 & 0,966 & 1,000 & 0,995 & 0,981 \\
$4(\mathrm{i}=5)$ & 0,997 & 0,966 & 0,997 & 1,000 & 0,980 \\
$4(\mathrm{i}=6)$ & 1,011 & 0,978 & 1,009 & 1,007 & 1,000 \\
$5(\mathrm{i}=2)$ & 0,999 & 0,965 & 0,997 & 0,994 & 0,980 \\
$5(\mathrm{i}=3)$ & 0,994 & 0,964 & 0,995 & 0,992 & 0,978 \\
$5(\mathrm{i}=4)$ & 0,998 & 0,967 & 1,000 & 0,995 & 0,981 \\
$5(\mathrm{i}=5)$ & 0,994 & 0,963 & 0,995 & 0,992 & 0,977 \\
$5(\mathrm{i}=6)$ & 0,999 & 0,967 & 0,999 & 0,996 & 0,984 \\
$6(\mathrm{i}=2)$ & 1,000 & 0,966 & 0,998 & 0,994 & 0,980 \\
$6(\mathrm{i}=3)$ & 1,009 & 1,000 & 1,008 & 1,007 & 0,994 \\
$6(\mathrm{i}=4)$ & 0,998 & 0,966 & 1,000 & 0,995 & 0,981 \\
$6(\mathrm{i}=5)$ & 0,995 & 0,964 & 0,996 & 1,000 & 0,979 \\
$6(\mathrm{i}=6)$ & 1,010 & 0,976 & 1,009 & 1,006 & 1,000 \\
\hline
\end{tabular}


Tabela 11: Resultados das estatísticas Wald e Razão de Verossimilhança(RV).

\begin{tabular}{rrrrr}
\hline & Wald & P-Valor & RV & P-Valor \\
hipóteses & & & & \\
\hline 1 & 1425,692 & 0,000 & 467,614 & 0,000 \\
2 & 224,282 & 0,000 & 135,211 & 0,000 \\
3 & 10,241 & 0,069 & 10,406 & 0,065 \\
$4(\mathrm{i}=2)$ & 3,501 & 0,174 & 3,487 & 0,175 \\
$4(\mathrm{i}=3)$ & 763,583 & 0,000 & 363,807 & 0,000 \\
$4(\mathrm{i}=4)$ & 6,069 & 0,048 & 6,391 & 0,041 \\
$4(\mathrm{i}=5)$ & 13,343 & 0,001 & 12,905 & 0,002 \\
$4(\mathrm{i}=6)$ & 236,608 & 0,000 & 176,491 & 0,000 \\
$5(\mathrm{i}=2)$ & 2,817 & 0,093 & 2,912 & 0,088 \\
$5(\mathrm{i}=3)$ & 0,161 & 0,688 & 0,160 & 0,689 \\
$5(\mathrm{i}=4)$ & 5,786 & 0,016 & 6,067 & 0,014 \\
$5(\mathrm{i}=5)$ & 0,024 & 0,876 & 0,024 & 0,876 \\
$5(\mathrm{i}=6)$ & 8,104 & 0,004 & 7,980 & 0,005 \\
$6(\mathrm{i}=2)$ & 3,491 & 0,062 & 3,487 & 0,062 \\
$6(\mathrm{i}=3)$ & 138,041 & 0,000 & 104,310 & 0,000 \\
$6(\mathrm{i}=4)$ & 4,074 & 0,044 & 4,149 & 0,042 \\
$6(\mathrm{i}=5)$ & 2,473 & 0,116 & 2,449 & 0,118 \\
$6(\mathrm{i}=6)$ & 73,847 & 0,000 & 62,471 & 0,000 \\
\hline
\end{tabular}

De acordo com a Tabela 11, segue que:

- para a hipótese $1\left(H_{0}: \alpha_{2}=\cdots=\alpha_{p}=0\right.$ e $\left.\beta_{2}=\cdots=\beta_{p}=1\right)$, concluímos que rejeita-se a hipótese nula para os dois testes (Wald e RV), ou seja, o grupo de motores não é consistente;

- para a hipótese $2\left(H_{0}: \beta_{2}=\cdots=\beta_{p}=1\right)$, concluímos que pelo menos um dos laboratórios está medindo com vício multiplicativo;

- para a hipótese $3\left(H_{0}: \alpha_{2}=\cdots=\alpha_{p}=0\right)$, adotando os níveis de significância 1 e 5\%, concluímos que não rejeita-se a hipótese nula para os dois testes, porém a 10\% rejeita-se a hipótese nula para os dois testes, ou seja, pelo menos um dos laboratórios está medindo com vício aditivo; 
- para a hipótese $4\left(H_{0}: \alpha_{i}=0\right.$ e $\left.\beta_{i}=1, i=2, \cdots, p\right)$, para o laboratório $i=2$ adotando os níveis de significância 1,5 e 10\%, não rejeita-se a hipótese nula para os dois testes, ou seja, este laboratório mede sem vícios nestes níveis; para os laboratórios $i=3,5,6$, concluímos que rejeita-se a hipótese nula para os dois testes, isto é, estes laboratórios não medem sem vícios; para o laboratório $i=4$, adotando os níveis de significância 5 e 10\%, concluímos que rejeita-se a hipótese nula para os dois testes, porém a $1 \%$ não rejeita-se a hipótese nula para os dois testes e portanto o laboratório mede sem vícios;

- para a hipótese $5\left(H_{0}: \alpha_{i}=0, i=2, \cdots, p\right)$, para o laboratório $i=2$, adotando os níveis de significância 1 e 5\%, não rejeita-se a hipótese nula para os dois testes, porém a 10\% a hipótese nula é rejeitada para os dois testes, portanto, mede com vícios aditivos; para os laboratórios $i=3,5$, adotando os níveis de significância 1, 5 e 10\% concluímos que não rejeita-se a hipótese nula para os dois testes, ou seja, estes laboratórios medem sem vícios aditivos; para o laboratório $i=6$, concluímos que rejeita-se a hipótese nula para os dois testes; para o laboratório $i=4$, concluímos que ao nível $1 \%$ não rejeita-se a hipótese nula para os dois testes, porém a 5 e 10\%, rejeita-se a hipótese nula para os dois testes, portanto o laboratório não mede sem vícios aditivos para estes níveis;

- para a hipótese $6\left(H_{0}: \beta_{i}=1, i=2, \cdots, p\right)$, para o laboratório $i=2$, adotando os níveis de significância 1 e 5\%, não rejeita-se a hipótese nula para os dois testes, porém a 10\% a hipótese nula é rejeitada para os dois testes, portanto, mede com vícios multiplicativos; para o laboratório $i=5$, adotando os níveis de significância 1,5 e 10\% concluímos que não rejeita-se a hipótese nula para os dois testes, ou seja, este laboratório mede sem vícios multiplicativos; para o laboratório $i=3,6$, concluímos que rejeita-se a hipótese nula para os dois testes, ou seja, estes laboratórios não medem sem vícios multiplicativos; para o laboratório $i=4$, concluímos que ao nível $1 \%$ não rejeita-se a hipótese nula 
para os dois testes, porém a 5 e 10\%, rejeita-se a hipótese nula para os dois testes, portanto o laboratório não mede sem vícios multiplicativos para estes níveis.

\subsubsection{Comparação dos resultados dos testes Normal e t de Student}

Apresentamos nesta seção a Tabela 12 com a interpretação dos p-valores obtidos nas estatísticas de Wald (os p-valores da estatística de razão de verossimilhança não foram apresentados nesta Tabela pois são muito próximos dos da estatística de Wald e portanto, a conclusão é a mesma) para as distribuições normal e t de student quando fixamos o nível de significância (nível de sig.) em 5\%. Nosso interesse aqui é poder comparar os laboratórios através dos testes e assim concluirmos qual deles está fazendo a melhor medição.

Tabela 12: Interpretação do p-valor para o nível fixado 5\% para as duas distribuições.

\begin{tabular}{rrrrr}
\hline & $\begin{array}{r}\text { Normal } \\
\text { P-valor }\end{array}$ & nível de sig. 5\% & $\begin{array}{r}\text { T de Student } \\
\text { P-valor }\end{array}$ & nível de sig. 5\% \\
Hipóteses & & & & \\
\hline 1 & 0,000 & rej hip nula & 0,000 & rej hip nula \\
3 & 0,000 & rej hip nula & 0,000 & rej hip nula \\
$4(\mathrm{i}=2)$ & 0,002 & rej hip nula & 0,069 & $\tilde{n}$ rej hip nula \\
$4(\mathrm{i}=3)$ & 0,086 & n rej hip nula & 0,174 & $\tilde{n}$ rej hip nula \\
$4(\mathrm{i}=4)$ & 0,000 & rej hip nula & 0,000 & rej hip nula \\
$4(\mathrm{i}=5)$ & 0,000 & rej hip nula & 0,048 & rej hip nula \\
$4(\mathrm{i}=6)$ & 0,007 & rej hip nula & 0,001 & rej hip nula \\
$5(\mathrm{i}=2)$ & 0,171 & rej hip nula & 0,000 & rej hip nula \\
$5(\mathrm{i}=3)$ & 0,320 & $\tilde{n}$ rej hip nula & 0,093 & $\tilde{n}$ rej hip nula \\
$5(\mathrm{i}=4)$ & 0,002 & rej hip nula & 0,688 & $\tilde{n}$ rej hip nula \\
$5(\mathrm{i}=5)$ & 0,868 & $\tilde{n}$ rej hip nula & 0,016 & rej hip nula \\
$5(\mathrm{i}=6)$ & 0,068 & $\tilde{n}$ rej hip nula & 0,004 & ñ rej hip nula \\
$6(\mathrm{i}=2)$ & 0,043 & rej hip nula & 0,062 & rej hip nula \\
$6(\mathrm{i}=3)$ & 0,000 & rej hip nula & 0,000 & rej hip nula \\
$6(\mathrm{i}=4)$ & 0,000 & rej hip nula & 0,044 & rej hip nula \\
$6(\mathrm{i}=5)$ & 0,167 & $\tilde{n}$ rej hip nula & 0,116 & $\tilde{n}$ rej hip nula \\
$6(\mathrm{i}=6)$ & 0,000 & rej hip nula & 0,000 & rej hip nula \\
\hline
\end{tabular}


Após analisarmos a Tabela 12, notamos que os laboratórios que não rejeitaram a hipótese nula usando a distribuição normal foram:

- o segundo, sob a quarta hipótese,

- o segundo, o terceiro, o quinto e o sexto, sob a quinta hipótese,

- o quinto, sob a sexta hipótese.

Usando a distribuição t de student:

- o segundo, sob a quarta hipótese,

- o segundo, o terceiro e o quinto, sob a quinta hipótese,

- o quinto, sob a sexta hipótese.

Desta forma, concluímos que o segundo e o quinto laboratório estão medindo melhor sob as duas distribuições. 


\section{Estudos de Simulação}

Neste Capítulo, apresentaremos um estudo de simulação para verificar o comportamento dos testes assintóticos de Wald e razão de verossimilhança quando consideramos diferentes valores para $\mathrm{p}, \mathrm{m}, n_{i}, i=1, \ldots, p$, nível de significância e parâmetros. Foram considerados diferentes valores para $\sigma_{x j}^{2}$ e $\sigma_{i j}^{2}$ e as possíveis combinações entre eles.

Os valores dos parâmetros considerados foram:

$\boldsymbol{\mu}_{\boldsymbol{x}}=\left(\mu_{x_{1}}, \ldots, \mu_{x_{m}}\right)=(19.717,27.318,35.562,45.680,54.540,63.552,71.320,76.693$, $79.831,79.790)$,

$$
\begin{aligned}
& \boldsymbol{\alpha}=\left(\alpha_{1}, \ldots, \alpha_{p}\right)=(0.000,0.224,-0.188,0.418,0.074,0.243) \\
& \boldsymbol{\beta}=\left(\beta_{1}, \ldots, \beta_{p}\right)=(1.000,0.0992,0.970,0.987,0.989,0.978) \\
& \boldsymbol{\sigma}_{\boldsymbol{x}}^{\mathbf{2}}=\left(\sigma_{x_{1}}^{2}, \ldots, \sigma_{x_{m}}^{2}\right)=(0.010,0.018,0.031,0.051,0.072,0.097,0.122,0.140,0.154
\end{aligned}
$$

$0.156)$,

$$
\begin{gathered}
\boldsymbol{\sigma}^{\mathbf{2}}=\left[\begin{array}{cccc}
\sigma_{11}^{2} & \sigma_{12}^{2} & \cdots & \sigma_{1 m}^{2} \\
\sigma_{21}^{2} & \sigma_{22}^{2} & \cdots & \sigma_{2 m}^{2} \\
\vdots & \vdots & \ddots & \vdots \\
\sigma_{p 1}^{2} & \sigma_{p 2}^{2} & \cdots & \sigma_{p m}^{2}
\end{array}\right]= \\
=\left[\begin{array}{cccccccccc}
0.038 & 0.067 & 0.114 & 0.188 & 0.267 & 0.371 & 0.472 & 0.532 & 0.580 & 0.620 \\
0.038 & 0.070 & 0.118 & 0.194 & 0.286 & 0.399 & 0.572 & 0.618 & 0.681 & 0.836 \\
0.098 & 0.126 & 0.162 & 0.217 & 0.276 & 0.325 & 0.385 & 0.439 & 0.461 & 0.466 \\
0.015 & 0.023 & 0.039 & 0.064 & 0.089 & 0.126 & 0.156 & 0.179 & 0.191 & 0.185 \\
1.616 & 1.170 & 1.188 & 1.211 & 1.233 & 1.261 & 1.288 & 1.302 & 1.315 & 1.306 \\
0.015 & 0.029 & 0.045 & 0.072 & 0.100 & 0.133 & 0.164 & 0.195 & 0.223 & 0.232
\end{array}\right],
\end{gathered}
$$

para $m=10$ e $p=6$, ou seja, os valores obtidos na aplicação. Representamos por $\sigma_{x_{j} s}^{2}$ valores menores do que $\sigma_{x_{j}}^{2}$ e por $\sigma_{x_{j} l}^{2}$, valores maiores. Analogamente, representamos por $\sigma_{i j s}^{2}$ valores menores do que $\sigma_{i j}^{2}$ e por $\sigma_{i j l}^{2}$, valores maiores. Os valores considerados foram: 


$$
\begin{aligned}
& \boldsymbol{\sigma}_{x s}^{2}=(0.001,0.002,0.003,0.005,0.007,0.009,0.01,0.014,0.015,0.016) \\
& \boldsymbol{\sigma}_{x l}^{2}=(0.030,0.056,0.095,0.157,0.223,0.300,0.377,0.434,0.477,0.484) \\
& \boldsymbol{\sigma}_{i s}^{2}=(0.010,0.020,0.040,0.060,0.090,0.120,0.160,0.180,0.190,0.180) \mathrm{e} \\
& \boldsymbol{\sigma}_{i l}^{2}=(0.150,0.290,0.450,0.720,0.990,0.133,0.164,0.195,2.230,2.31)
\end{aligned}
$$

para $m=10$ e $p=6$. Na simulação também foram consideradas as possíveis combinações entre os valores descritos anteriormente.

Primeiro foi feito um estudo do valor empírico em relação ao nível de significância do teste. Foram considerados os níveis 1\%, 5\% e $10 \%$ e calculadas as porcentagens de rejeição sob 5000 amostras geradas; calculamos os valores considerando 3, 5 e 20 réplicas. Os resultados foram feitos para dois casos, o primeiro considera 6 laboratórios e 10 pontos de rotação, o segundo, 3 laboratórios e 5 pontos de rotação (Apêndice B). Neste caso, foram considerados os mesmos valores dos parâmetros colocados para o primeiro caso, mas lembrando que $i=1, \ldots, p$ e $j=1, \ldots, m$, e agora, $p=3$ e $m=5$, então consideramos os valores somente para $i=1,3$ e 5 e $j=1,3,5,7$ e 9 .

Em seguida, fizemos a simulação do poder do teste que calculou a porcentagem de rejeição de 5000 amostras sob as hipóteses alternativas $H_{1}$; os valores obtidos são apresentados no Apêndice C. Para tanto foi considerado um distanciamento gradativo $\delta$ que variou de acordo com as hipóteses testadas. Para as hipóteses 1, 2 e 3, esse distanciamento foi feito em apenas um dos laboratórios, o segundo.

Neste estudo foi considerado o nível de $5 \%$, e foi feito para os dois casos discutidos acima.

Os estudos de simulação foram feitos sob duas distribuições: Normal e t de Student. Para o modelo t de Student, consideramos o grau de liberdade $=3.4$.

Uma observação a ser feita é que para as hipóteses 4,5 e 6, os resultados apresentados se referem somente ao laboratório 2.

A seguir, apresentaremos os resultados da simulação para o valor empírico e para o poder do teste para as distribuições Normal e t de Student para 6 laboratórios e 10 rotações (ou seja, valores da aplicação). Os demais resultados podem ser encontrados 
nos Apêndices B e C.

\subsection{Resultados da simulação para o valor empírico: distribui- ções Normal e t de Student}

Na sequência, as tabelas com os resultados da simulação do valor empírico para a distribuição Normal:

Tabela 13: Tamanhos empíricos dos testes de Wald e Razão de Verossimilhança para a primeira hipótese com $\mathrm{p}=6$ e $\mathrm{m}=10$ - Distribuição Normal.

\begin{tabular}{|c|c|c|c|c|c|c|c|c|c|c|c|}
\hline \multirow[b]{2}{*}{ nivel } & \multirow[b]{2}{*}{ teste } & \multirow[b]{2}{*}{$\mathrm{ni}$} & \multicolumn{3}{|c|}{$\sigma_{x_{j s}}^{2}$} & \multicolumn{3}{|c|}{$\sigma_{x_{j}}^{2}$} & \multicolumn{3}{|c|}{$\sigma_{x_{j l}}^{2}$} \\
\hline & & & $\sigma_{s}^{2}$ & $\sigma^{2}$ & $\sigma_{l}^{2}$ & $\sigma_{s}^{2}$ & $\sigma^{2}$ & $\sigma_{l}^{2}$ & $\sigma_{s}^{2}$ & $\sigma^{2}$ & $\sigma_{l}^{2}$ \\
\hline \multirow[t]{6}{*}{0,01} & W & 3 & 0,0092 & 0,0093 & 0,0082 & 0,0081 & 0,0091 & 0,009 & 0,0094 & 0,0096 & 0,009 \\
\hline & W & 5 & 0,0094 & 0,0096 & 0,0084 & 0,0092 & 0,0094 & 0,0092 & 0,0096 & 0,0099 & 0,0092 \\
\hline & W & 20 & 0,0099 & 0,010 & 0,009 & 0,0099 & 0,0098 & 0,0099 & 0,010 & 0,010 & 0,0098 \\
\hline & RV & 3 & 0,0086 & 0,009 & 0,008 & 0,008 & 0,0089 & 0,0085 & 0,009 & 0,0094 & 0,0089 \\
\hline & RV & 5 & 0,0089 & 0,0094 & 0,0086 & 0,0092 & 0,009 & 0,0088 & 0,0094 & 0,0098 & 0,009 \\
\hline & RV & 20 & 0,0099 & 0,0099 & 0,0089 & 0,0098 & 0,010 & 0,0098 & 0,010 & 0,010 & 0,0099 \\
\hline \multirow[t]{6}{*}{0,05} & W & 3 & 0,0469 & 0,0480 & 0,0490 & 0,0461 & 0,0488 & 0,0489 & 0,049 & 0,0484 & 0,0489 \\
\hline & W & 5 & 0,0478 & 0,0484 & 0,0496 & 0,0464 & 0,049 & 0,0498 & 0,0493 & 0,0486 & 0,0492 \\
\hline & W & 20 & 0,0498 & 0,0498 & 0,05 & 0,0496 & 0,05 & 0,050 & 0,0499 & 0,0495 & 0,05 \\
\hline & RV & 3 & 0,0468 & 0,048 & 0,0486 & 0,0470 & 0,0482 & 0,0479 & 0,047 & 0,0492 & 0,049 \\
\hline & RV & 5 & 0,0477 & 0,049 & 0,0492 & 0,0472 & 0,0484 & 0,0488 & 0,0489 & 0,0494 & 0,0493 \\
\hline & RV & 20 & 0,0499 & 0,0496 & 0,0498 & 0,0493 & 0,0498 & 0,0499 & 0,0498 & 0,0499 & 0,05 \\
\hline \multirow[t]{6}{*}{0,1} & W & 3 & 0,0979 & 0,089 & 0,0959 & 0,09 & 0,088 & 0,091 & 0,093 & 0,0921 & 0,090 \\
\hline & W & 5 & 0,098 & 0,099 & 0,0962 & 0,0902 & 0,099 & 0,0942 & 0,096 & 0,0946 & 0,092 \\
\hline & W & 20 & 0,099 & 0,1 & 0,0986 & 0,0981 & 0,1 & 0,0993 & 0,099 & 0,0983 & 0,099 \\
\hline & RV & 3 & & 0,0980 & & 0,09 & 0,099 & 0,0924 & 0,0935 & 0,0943 & 0,093 \\
\hline & RV & 5 & 0,0987 & 0,0986 & 0,0956 & 0,0904 & 0,0992 & 0,0946 & 0,097 & 0,0954 & 0,098 \\
\hline & RV & 20 & 0,0999 & 0,099 & 0,0989 & 0,098 & 0,10 & 0,098 & 0,099 & 0,0989 & 0,0999 \\
\hline
\end{tabular}


Tabela 14: Tamanhos empíricos dos testes de Wald e Razão de Verossimilhança para a segunda hipótese com $\mathrm{p}=6$ e m=10 - Distribuição Normal.

\begin{tabular}{|c|c|c|c|c|c|c|c|c|c|c|c|}
\hline \multirow[b]{2}{*}{ nivel } & \multirow[b]{2}{*}{ teste } & \multirow[b]{2}{*}{ ni } & \multicolumn{3}{|c|}{$\sigma_{x_{i s}}^{2}$} & \multicolumn{3}{|c|}{$\sigma_{x}^{2}$} & \multicolumn{3}{|c|}{$\sigma_{x_{i l}}^{2}$} \\
\hline & & & $\sigma_{s}^{2}$ & $\begin{array}{r}x_{j s} \\
\sigma^{2}\end{array}$ & $\sigma_{l}^{2}$ & $\sigma_{s}^{2}$ & $\sigma_{j}^{2}$ & $\sigma_{l}^{2}$ & $\sigma_{s}^{2}$ & $\sigma_{j l}^{x_{j}} \sigma^{2}$ & $\sigma_{l}^{2}$ \\
\hline \multirow[t]{6}{*}{0,01} & $\mathrm{~W}$ & 3 & 0,0084 & 0,0082 & 0,0086 & 0,0092 & 0,0090 & 0,0079 & 0,0093 & 0,0092 & 0,0096 \\
\hline & W & 5 & 0,0088 & 0,0086 & 0,0093 & 0,0096 & 0,0098 & 0,0084 & 0,0099 & 0,0098 & 0,0098 \\
\hline & W & 20 & 0,0098 & 0,0098 & 0,0099 & 0,0099 & 0,010 & 0,0096 & 0,010 & 0,010 & 0,010 \\
\hline & RV & 3 & 0,0082 & 0,0080 & 0,0084 & 0,0089 & 0,0089 & 0,0081 & 0,0090 & 0,0090 & 0,0092 \\
\hline & RV & 5 & 0,0084 & 0,0084 & 0,0096 & 0,0093 & 0,0092 & 0,0084 & 0,0094 & 0,0096 & 0,0098 \\
\hline & RV & 20 & 0,0097 & 0,0099 & 0,010 & 0,0099 & 0,0099 & 0,0099 & 0,01 & 0,0099 & 0,010 \\
\hline \multirow[t]{6}{*}{0,05} & W & 3 & 0,0482 & 0,0470 & 0,0474 & 0,0479 & 0,047 & 0,0484 & 0,0490 & 0,0476 & 0,0481 \\
\hline & W & 5 & 0,0496 & 0,0484 & 0,0486 & 0,0482 & 0,0494 & 0,0492 & 0,0497 & 0,0480 & 0,0493 \\
\hline & W & 20 & 0,050 & 0,049 & 0,0499 & 0,049 & 0,05 & 0,0499 & 0,05 & 0,049 & 0,0499 \\
\hline & RV & 3 & 0,0480 & 0,0469 & 0,0482 & 0,0473 & 0,0478 & 0,0484 & 0,0490 & 0,0472 & 0,0490 \\
\hline & RV & 5 & 0,0494 & 0,0484 & 0,0494 & 0,0484 & 0,0484 & 0,0494 & 0,0496 & 0,0478 & 0,0498 \\
\hline & RV & 20 & 0,0499 & 0,0498 & 0,0499 & 0,049 & 0,05 & 0,05 & 0,05 & 0,0498 & 0,05 \\
\hline \multirow[t]{6}{*}{0,1} & W & 3 & 0,0972 & 0,0974 & 0,0941 & 0,0976 & 0,0981 & 0,0989 & 0,089 & 0,098 & 0,090 \\
\hline & W & 5 & 0,0983 & 0,0972 & 0,0986 & 0,0982 & 0,0984 & 0,0998 & 0,096 & 0,0996 & 0,099 \\
\hline & W & 20 & 0,0999 & 0,0993 & 0,0993 & 0,0997 & 0,099 & 0,10 & 0,099 & 0,10 & 0,10 \\
\hline & RV & 3 & 0,0970 & 0,0977 & 0,0943 & 0,0973 & 0,093 & 0,0983 & 0,088 & 0,098 & 0,088 \\
\hline & RV & 5 & 0,0984 & 0,0982 & 0,0974 & 0,0988 & 0,099 & 0,0994 & 0,099 & 0,0992 & 0,097 \\
\hline & RV & 20 & 0,0999 & 0,099 & 0,0989 & 0,0989 & 0,10 & 0,10 & 0,10 & 0,099 & 0,10 \\
\hline
\end{tabular}


Tabela 15: Tamanhos empíricos dos testes de Wald e Razão de Verossimilhança para a terceira hipótese com $\mathrm{p}=6$ e m=10 - Distribuição Normal.

\begin{tabular}{|c|c|c|c|c|c|c|c|c|c|c|c|}
\hline \multirow[b]{2}{*}{ nivel } & \multirow[b]{2}{*}{ teste } & \multirow[b]{2}{*}{ ni } & \multicolumn{3}{|c|}{$\sigma_{x_{i s}}^{2}$} & \multicolumn{3}{|c|}{$\sigma_{x}^{2}$} & \multicolumn{3}{|c|}{$\sigma_{x_{i l}}^{2}$} \\
\hline & & & $\sigma_{s}^{2}$ & $\begin{array}{r}x_{j s} \\
\sigma^{2}\end{array}$ & $\sigma_{l}^{2}$ & $\sigma_{s}^{2}$ & $\sigma_{j}^{2}$ & $\sigma_{l}^{2}$ & $\sigma_{s}^{2}$ & $\sigma_{j l}^{x_{j}} \sigma^{2}$ & $\sigma_{l}^{2}$ \\
\hline \multirow[t]{6}{*}{0,01} & $\mathrm{~W}$ & 3 & 0,0091 & 0,0092 & 0,0082 & 0,0088 & 0,0089 & 0,0093 & 0,0080 & 0,0089 & 0,0088 \\
\hline & W & 5 & 0,0094 & 0,0095 & 0,0086 & 0,009 & 0,0092 & 0,0098 & 0,0084 & 0,0092 & 0,0092 \\
\hline & W & 20 & 0,0099 & 0,0099 & 0,0098 & 0,0099 & 0,0098 & 0,010 & 0,0098 & 0,0099 & 0,0099 \\
\hline & RV & 3 & 0,0091 & 0,0090 & 0,0081 & 0,0086 & 0,0089 & 0,0091 & 0,0079 & 0,0088 & 0,0090 \\
\hline & RV & 5 & 0,0093 & 0,0096 & 0,0086 & 0,009 & 0,009 & 0,0096 & 0,0082 & 0,0092 & 0,0096 \\
\hline & RV & 20 & 0,0097 & 0,010 & 0,0096 & 0,0098 & 0,010 & 0,0098 & 0,0096 & 0,0099 & 0,10 \\
\hline \multirow[t]{6}{*}{0,05} & W & 3 & 0,0484 & 0,0478 & 0,0489 & 0,0484 & 0,0468 & 0,0479 & 0,0467 & 0,0489 & 0,0492 \\
\hline & W & 5 & 0,0492 & 0,0489 & 0,0498 & 0,0494 & 0,0479 & 0,0488 & 0,0488 & 0,049 & 0,0499 \\
\hline & W & 20 & 0,0499 & 0,0499 & 0,05 & 0,0499 & 0,0499 & 0,0499 & 0,0495 & 0,05 & 0,05 \\
\hline & RV & 3 & 0,0480 & 0,0473 & 0,0485 & 0,048 & 0,0462 & 0,0471 & 0,0481 & 0,0480 & 0,0490 \\
\hline & RV & 5 & 0,0491 & 0,0498 & 0,0496 & 0,049 & 0,0484 & 0,0484 & 0,0488 & 0,0486 & 0,0496 \\
\hline & RV & 20 & 0,0499 & 0,05 & 0,05 & 0,05 & 0,0499 & 0,0497 & 0,0498 & 0,0499 & 0,05 \\
\hline \multirow[t]{6}{*}{0,1} & W & 3 & 0,0979 & 0,088 & 0,0980 & 0,089 & 0,0848 & 0,0931 & 0,0921 & 0,0967 & 0,090 \\
\hline & W & 5 & 0,0986 & 0,093 & 0,0984 & 0,099 & 0,0929 & 0,0942 & 0,0984 & 0,0982 & 0,094 \\
\hline & W & 20 & 0,099 & 0,099 & 0,099 & 0,10 & 0,099 & 0,099 & 0,099 & 0,099 & 0,10 \\
\hline & RV & 3 & 0,0977 & 0,088 & 0,0978 & 0,084 & 0,0939 & 0,094 & 0,0954 & 0,0989 & 0,092 \\
\hline & RV & 5 & 0,0983 & 0,097 & 0,0986 & 0,0992 & 0,0992 & 0,0946 & 0,0994 & 0,0994 & 0,096 \\
\hline & RV & 20 & 0,0989 & 0,10 & 0,099 & 0,0999 & 0,10 & 0,098 & 0,099 & 0,0999 & 0,10 \\
\hline
\end{tabular}


Tabela 16: Tamanhos empíricos dos testes de Wald e Razão de Verossimilhança para a quarta hipótese com $\mathrm{p}=6$ e $\mathrm{m}=10$ - Distribuição Normal.

\begin{tabular}{|c|c|c|c|c|c|c|c|c|c|c|c|}
\hline \multirow[b]{2}{*}{ nivel } & \multirow[b]{2}{*}{ teste } & \multirow[b]{2}{*}{ ni } & \multicolumn{3}{|c|}{$\sigma_{x_{i s}}^{2}$} & \multicolumn{3}{|c|}{$\sigma_{x}^{2}$} & \multicolumn{3}{|c|}{$\sigma_{x_{j i}}^{2}$} \\
\hline & & & $\sigma_{s}^{2}$ & $\begin{array}{r}x_{j s} \\
\sigma^{2}\end{array}$ & $\sigma_{l}^{2}$ & $\sigma_{s}^{2}$ & $\sigma_{j}^{2}$ & $\sigma_{l}^{2}$ & $\sigma_{s}^{2}$ & $\sigma_{j l}^{x_{j}} \sigma^{2}$ & $\sigma_{l}^{2}$ \\
\hline \multirow[t]{6}{*}{0,01} & $\mathrm{~W}$ & 3 & 0,0082 & 0,0083 & 0,0091 & 0,0092 & 0,0089 & 0,0079 & 0,0094 & 0,0088 & 0,0092 \\
\hline & W & 5 & 0,0093 & 0,0091 & 0,0098 & 0,0096 & 0,0094 & 0,0082 & 0,0098 & 0,0094 & 0,0096 \\
\hline & W & 20 & 0,0098 & 0,0098 & 0,10 & 0,0099 & 0,0099 & 0,0099 & 0,10 & 0,0099 & 0,10 \\
\hline & RV & 3 & 0,0080 & 0,0082 & 0,0088 & 0,009 & 0,0088 & 0,0080 & 0,0097 & 0,0089 & 0,0090 \\
\hline & RV & 5 & 0,0094 & 0,0092 & 0,0092 & 0,0093 & 0,0092 & 0,0084 & 0,0099 & 0,0092 & 0,0098 \\
\hline & RV & 20 & 0,0099 & 0,0099 & 0,0099 & 0,0098 & 0,0098 & 0,0099 & 0,10 & 0,0098 & 0,10 \\
\hline \multirow[t]{6}{*}{0,05} & W & 3 & 0,0479 & 0,0469 & 0,0485 & 0,0489 & 0,0474 & 0,0484 & 0,0490 & 0,0476 & 0,0494 \\
\hline & W & 5 & 0,0486 & 0,0484 & 0,0496 & 0,0496 & 0,0486 & 0,0494 & 0,0499 & 0,0482 & 0,0499 \\
\hline & W & 20 & 0,0499 & 0,0497 & 0,050 & 0,0499 & 0,0498 & 0,050 & 0,050 & 0,0499 & 0,050 \\
\hline & RV & 3 & 0,0474 & 0,0476 & 0,0476 & 0,0487 & 0,0472 & 0,0482 & 0,048 & 0,0474 & 0,0488 \\
\hline & RV & 5 & 0,0482 & 0,0486 & 0,0488 & 0,0498 & 0,0484 & 0,0498 & 0,049 & 0,0489 & 0,049 \\
\hline & RV & 20 & 0,0489 & 0,0499 & 0,0499 & 0,050 & 0,0499 & 0,050 & 0,050 & 0,05 & 0,050 \\
\hline \multirow[t]{6}{*}{0,1} & W & 3 & 0,0984 & 0,0987 & 0,091 & 0,0913 & 0,0961 & 0,0931 & 0,094 & 0,0879 & 0,0936 \\
\hline & W & 5 & 0,0992 & 0,0996 & 0,0992 & 0,0934 & 0,0988 & 0,0982 & 0,096 & 0,0989 & 0,0982 \\
\hline & W & 20 & 0,0999 & 0,10 & 0,10 & 0,0989 & 0,0999 & 0,099 & 0,099 & 0,10 & 0,0992 \\
\hline & RV & 3 & 0,098 & 0,0989 & 0,088 & 0,0925 & 0,0953 & 0,090 & 0,089 & 0,0864 & 0,091 \\
\hline & RV & 5 & 0,099 & 0,0993 & 0,099 & 0,0977 & 0,0994 & 0,0972 & 0,092 & 0,0982 & 0,0975 \\
\hline & RV & 20 & 0,10 & 0,0999 & 0,10 & 0,099 & 0,10 & 0,10 & 0,099 & 0,0999 & 0,099 \\
\hline
\end{tabular}


Tabela 17: Tamanhos empíricos dos testes de Wald e Razão de Verossimilhança para a quinta hipótese com $\mathrm{p}=6$ e $\mathrm{m}=10$ - Distribuição Normal.

\begin{tabular}{|c|c|c|c|c|c|c|c|c|c|c|c|}
\hline \multirow[b]{2}{*}{ nivel } & \multirow[b]{2}{*}{ teste } & \multirow[b]{2}{*}{ ni } & \multicolumn{3}{|c|}{$\sigma_{x_{i s}}^{2}$} & \multicolumn{3}{|c|}{$\sigma_{x_{i}}^{2}$} & \multicolumn{3}{|c|}{$\sigma_{x_{j i}}^{2}$} \\
\hline & & & $\sigma_{s}^{2}$ & $\begin{array}{r}x_{j s} \\
\sigma^{2}\end{array}$ & $\sigma_{l}^{2}$ & $\sigma_{s}^{2}$ & $\sigma_{j}^{2}$ & $\sigma_{l}^{2}$ & $\sigma_{s}^{2}$ & $\sigma_{j l}^{x_{j}} \sigma^{2}$ & $\sigma_{l}^{2}$ \\
\hline \multirow[t]{6}{*}{0,01} & $\mathrm{~W}$ & 3 & 0,0086 & 0,0089 & 0,0093 & 0,0084 & 0,0092 & 0,0089 & 0,0091 & 0,0084 & 0,0089 \\
\hline & W & 5 & 0,0090 & 0,0092 & 0,0096 & 0,0090 & 0,0096 & 0,0094 & 0,0094 & 0,009 & 0,0096 \\
\hline & W & 20 & 0,0099 & 0,010 & 0,0099 & 0,0099 & 0,0099 & 0,0099 & 0,0098 & 0,010 & 0,010 \\
\hline & RV & 3 & 0,0084 & 0,0087 & 0,0091 & 0,0080 & 0,0090 & 0,0088 & 0,0089 & 0,0087 & 0,0088 \\
\hline & RV & 5 & 0,0086 & 0,0094 & 0,0094 & 0,0086 & 0,0092 & 0,0090 & 0,0091 & 0,0092 & 0,0092 \\
\hline & RV & 20 & 0,0099 & 0,10 & 0,0098 & 0,0099 & 0,0098 & 0,0098 & 0,0097 & 0,0099 & 0,010 \\
\hline \multirow[t]{6}{*}{0,05} & W & 3 & 0,0486 & 0,0489 & 0,0476 & 0,0481 & 0,0468 & 0,0481 & 0,0474 & 0,0488 & 0,0491 \\
\hline & W & 5 & 0,0489 & 0,0499 & 0,0484 & 0,0488 & 0,0485 & 0,0496 & 0,0496 & 0,0498 & 0,0499 \\
\hline & W & 20 & 0,050 & 0,050 & 0,0499 & 0,0499 & 0,0496 & 0,0499 & 0,05 & 0,050 & 0,050 \\
\hline & RV & 3 & 0,0484 & 0,048 & 0,0484 & 0,0480 & 0,0465 & 0,0472 & 0,0478 & 0,0485 & 0,0492 \\
\hline & RV & 5 & 0,0488 & 0,049 & 0,0499 & 0,0484 & 0,0483 & 0,0484 & 0,0486 & 0,0496 & 0,0498 \\
\hline & RV & 20 & 0,0499 & 0,050 & 0,05 & 0,0499 & 0,0499 & 0,0496 & 0,0499 & 0,0499 & 0,0499 \\
\hline \multirow[t]{6}{*}{0,1} & W & 3 & 0,0974 & 0,0984 & 0,0973 & 0,0944 & 0,0965 & 0,0974 & 0,0976 & 0,0945 & 0,093 \\
\hline & W & 5 & 0,0983 & 0,099 & 0,0994 & 0,0956 & 0,0984 & 0,0989 & 0,0981 & 0,0968 & 0,0979 \\
\hline & W & 20 & 0,0999 & 0,10 & 0,10 & 0,099 & 0,10 & 0,10 & 0,0999 & 0,099 & 0,10 \\
\hline & RV & 3 & 0,0972 & 0,097 & 0,096 & 0,094 & 0,0964 & 0,0970 & 0,0984 & 0,0954 & 0,090 \\
\hline & RV & 5 & 0,0982 & 0,0992 & 0,0978 & 0,0962 & 0,0982 & 0,0992 & 0,0999 & 0,0962 & 0,0962 \\
\hline & RV & 20 & 0,0999 & 0,10 & 0,099 & 0,099 & 0,099 & 0,10 & 0,10 & 0,10 & 0,0999 \\
\hline
\end{tabular}


Tabela 18: Tamanhos empíricos dos testes de Wald e Razão de Verossimilhança para a sexta hipótese com $\mathrm{p}=6$ e $\mathrm{m}=10$ - Distribuição Normal.

\begin{tabular}{|c|c|c|c|c|c|c|c|c|c|c|c|}
\hline \multirow[b]{2}{*}{ nivel } & \multirow[b]{2}{*}{ teste } & \multirow[b]{2}{*}{ ni } & \multicolumn{3}{|c|}{$\sigma_{x_{i s}}^{2}$} & \multicolumn{3}{|c|}{$\sigma_{x_{i}}^{2}$} & \multicolumn{3}{|c|}{$\sigma_{x_{j i}}^{2}$} \\
\hline & & & $\sigma_{s}^{2}$ & $\begin{array}{r}x_{j s} \\
\sigma^{2}\end{array}$ & $\sigma_{l}^{2}$ & $\sigma_{s}^{2}$ & $\sigma_{j}^{2}$ & $\sigma_{l}^{2}$ & $\sigma_{s}^{2}$ & $\sigma_{j l}^{x_{j}} \sigma^{2}$ & $\sigma_{l}^{2}$ \\
\hline \multirow[t]{6}{*}{0,01} & $\mathrm{~W}$ & 3 & 0,0081 & 0,0082 & 0,0098 & 0,0091 & 0,0081 & 0,0096 & 0,0094 & 0,0090 & 0,0080 \\
\hline & W & 5 & 0,0083 & 0,0086 & 0,0099 & 0,0095 & 0,0084 & 0,0099 & 0,0098 & 0,0099 & 0,0084 \\
\hline & W & 20 & 0,0098 & 0,0099 & 0,010 & 0,0099 & 0,0099 & 0,010 & 0,10 & 0,010 & 0,0099 \\
\hline & RV & 3 & 0,0079 & 0,0082 & 0,0094 & 0,0092 & 0,0079 & 0,0092 & 0,0090 & 0,0088 & 0,0078 \\
\hline & RV & 5 & 0,0084 & 0,0086 & 0,0098 & 0,0095 & 0,0084 & 0,0098 & 0,0094 & 0,0098 & 0,0084 \\
\hline & RV & 20 & 0,0098 & 0,0099 & 0,010 & 0,0099 & 0,10 & 0,0099 & 0,0099 & 0,010 & 0,0098 \\
\hline \multirow[t]{6}{*}{0,05} & W & 3 & 0,0484 & 0,0477 & 0,0461 & 0,0487 & 0,0484 & 0,0474 & 0,0480 & 0,0484 & 0,0458 \\
\hline & W & 5 & 0,0498 & 0,048 & 0,0482 & 0,047 & 0,0488 & 0,0496 & 0,0489 & 0,0486 & 0,0489 \\
\hline & W & 20 & 0,050 & 0,050 & 0,0499 & 0,0498 & 0,050 & 0,05 & 0,050 & 0,0499 & 0,050 \\
\hline & RV & 3 & 0,0473 & 0,047 & 0,0463 & 0,0468 & 0,047 & 0,0478 & 0,0488 & 0,0481 & 0,0442 \\
\hline & RV & 5 & 0,0489 & 0,0484 & 0,0484 & 0,0474 & 0,0488 & 0,0494 & 0,0499 & 0,0494 & 0,0472 \\
\hline & RV & 20 & 0,050 & 0,0499 & 0,0499 & 0,0499 & 0,050 & 0,05 & 0,050 & 0,050 & 0,0499 \\
\hline \multirow[t]{6}{*}{0,1} & W & 3 & 0,0989 & 0,0980 & 0,0942 & 0,0933 & 0,098 & 0,0961 & 0,096 & 0,0922 & 0,0963 \\
\hline & W & 5 & 0,0993 & 0,0988 & 0,0952 & 0,095 & 0,0996 & 0,0974 & 0,0978 & 0,0946 & 0,0988 \\
\hline & W & 20 & 0,099 & 0,10 & 0,098 & 0,098 & 0,10 & 0,0989 & 0,10 & 0,099 & 0,10 \\
\hline & RV & 3 & 0,098 & 0,0980 & 0,0940 & 0,0920 & 0,098 & 0,0963 & 0,093 & 0,091 & 0,095 \\
\hline & RV & 5 & 0,0991 & 0,0992 & 0,0958 & 0,0948 & 0,0998 & 0,0989 & 0,0972 & 0,0954 & 0,0980 \\
\hline & RV & 20 & 0,099 & 0,10 & 0,099 & 0,098 & 0,10 & 0,099 & 0,099 & 0,10 & 0,099 \\
\hline
\end{tabular}

A seguir, são apresentadas as tabelas com os resultados da simulação para o valor empírico com a distribuição t de Student. 
Tabela 19: Tamanhos empíricos dos testes de Wald e Razão de Verossimilhança para a primeira hipótese com $\mathrm{p}=6$ e $\mathrm{m}=10$ - Distribuição t de Student.

\begin{tabular}{|c|c|c|c|c|c|c|c|c|c|c|c|}
\hline \multirow[b]{2}{*}{ nivel } & \multirow[b]{2}{*}{ teste } & \multirow[b]{2}{*}{ ni } & \multicolumn{3}{|c|}{$\sigma_{x_{i s}}^{2}$} & \multicolumn{3}{|c|}{$\sigma_{x_{i}}^{2}$} & \multicolumn{3}{|c|}{$\sigma_{x_{i l}}^{2}$} \\
\hline & & & $\sigma_{s}^{2}$ & $\begin{array}{r}x_{j s} \\
\sigma^{2}\end{array}$ & $\sigma_{l}^{2}$ & $\sigma_{s}^{2}$ & $\sigma_{j}^{2}$ & $\sigma_{l}^{2}$ & $\sigma_{s}^{2}$ & $\sigma^{2}$ & $\sigma_{l}^{2}$ \\
\hline \multirow[t]{6}{*}{0,01} & $\mathrm{~W}$ & 3 & 0,0116 & 0,0118 & 0,0108 & 0,0184 & 0,0116 & 0,0119 & 0,0113 & 0,0114 & 0,0105 \\
\hline & W & 5 & 0,0103 & 0,0114 & 0,0104 & 0,0102 & 0,0105 & 0,0102 & 0,0101 & 0,0101 & 0,0102 \\
\hline & W & 20 & 0,0100 & 0,0101 & 0,0100 & 0,010 & 0,0100 & 0,010 & 0,010 & 0,010 & 0,0100 \\
\hline & RV & 3 & 0,0113 & 0,011 & 0,0114 & 0,0106 & 0,0118 & 0,0119 & 0,0118 & 0,0116 & 0,0107 \\
\hline & RV & 5 & 0,0109 & 0,0108 & 0,0111 & 0,0101 & 0,0102 & 0,0106 & 0,0106 & 0,0101 & 0,0101 \\
\hline & RV & 20 & 0,0101 & 0,0100 & 0,0101 & 0,010 & 0,010 & 0,010 & 0,010 & 0,0100 & 0,0100 \\
\hline \multirow[t]{6}{*}{0,05} & W & 3 & 0,0512 & 0,0512 & 0,0512 & 0,0517 & 0,0513 & 0,0512 & 0,0513 & 0,0512 & 0,0514 \\
\hline & W & 5 & 0,0509 & 0,0511 & 0,0508 & 0,0513 & 0,0512 & 0,051 & 0,0511 & 0,051 & 0,051 \\
\hline & W & 20 & 0,05 & 0,0500 & 0,050 & 0,0501 & 0,0501 & 0,0500 & 0,0501 & 0,050 & 0,0501 \\
\hline & RV & 3 & 0,0511 & 0,051 & 0,051 & 0,0513 & 0,0511 & 0,0511 & 0,0512 & 0,0516 & 0,0514 \\
\hline & RV & 5 & 0,0506 & 0,0501 & 0,0501 & 0,0509 & 0,0509 & 0,0508 & 0,0511 & 0,0512 & 0,0511 \\
\hline & RV & 20 & 0,0500 & 0,050 & 0,050 & 0,0500 & 0,0500 & 0,0500 & 0,0501 & 0,0501 & 0,0500 \\
\hline \multirow[t]{6}{*}{0,10} & W & 3 & 0,116 & 0,11 & 0,1094 & 0,1092 & 0,1134 & 0,1102 & 0,1018 & 0,1014 & 0,1026 \\
\hline & W & 5 & 0,1032 & 0,1012 & 0,103 & 0,1061 & 0,1071 & 0,102 & 0,101 & 0,101 & 0,1012 \\
\hline & W & 20 & 0,1003 & 0,1001 & 0,1002 & 0,1003 & 0,1009 & 0,10 & 0,10 & 0,10 & 0,1001 \\
\hline & RV & 3 & 0,1072 & 0,1076 & 0,1084 & 0,1072 & 0,1046 & 0,1106 & 0,1074 & 0,1107 & 0,1084 \\
\hline & RV & 5 & 0,1031 & 0,1053 & 0,1062 & 0,1043 & 0,1022 & 0,109 & 0,1033 & 0,104 & 0,101 \\
\hline & RV & 20 & 0,1002 & 0,1003 & 0,1009 & 0,1001 & 0,10 & 0,1007 & 0,10 & 0,1003 & 0,1001 \\
\hline
\end{tabular}


Tabela 20: Tamanhos empíricos dos testes de Wald e Razão de Verossimilhança para a segunda hipótese com $\mathrm{p}=6$ e $\mathrm{m}=10$ - Distribuição t de Student.

\begin{tabular}{|c|c|c|c|c|c|c|c|c|c|c|c|}
\hline \multirow[b]{2}{*}{ nivel } & \multirow[b]{2}{*}{ teste } & \multirow[b]{2}{*}{ ni } & \multicolumn{3}{|c|}{$\sigma_{x_{j s}}^{2}$} & \multicolumn{3}{|c|}{$\sigma_{r .}^{2}$} & \multicolumn{3}{|c|}{$\sigma_{x_{j l}}^{2}$} \\
\hline & & & $\sigma_{s}^{2}$ & $\begin{array}{r}\sigma_{j}^{s} \\
\sigma^{2}\end{array}$ & $\sigma_{l}^{2}$ & $\sigma_{s}^{2}$ & $\sigma^{2}$ & $\sigma_{l}^{2}$ & $\sigma_{s}^{2}$ & $\sigma^{2}$ & $\sigma_{l}^{2}$ \\
\hline \multirow[t]{6}{*}{0,01} & W & 3 & 0,0119 & 0,0107 & 0,0117 & 0,0113 & 0,0109 & 0,0108 & 0,0111 & 0,0116 & 0,0118 \\
\hline & W & 5 & 0,0108 & 0,0102 & 0,0109 & 0,0109 & 0,0101 & 0,0101 & 0,0109 & 0,0107 & 0,0109 \\
\hline & W & 20 & 0,0100 & 0,10 & 0,0100 & 0,0100 & 0,01 & 0,01 & 0,0100 & 0,0100 & 0,0100 \\
\hline & $\mathrm{RV}$ & 3 & 0,0118 & 0,0104 & 0,0113 & 0,011 & 0,0107 & 0,0106 & 0,011 & 0,0113 & 0,0114 \\
\hline & $\mathrm{RV}$ & 5 & 0,0106 & 0,0101 & 0,0109 & 0,0103 & 0,0102 & 0,0101 & 0,0109 & 0,0104 & 0,0109 \\
\hline & $\mathrm{RV}$ & 20 & 0,0100 & 0,010 & 0,0100 & 0,0100 & 0,010 & 0,010 & 0,0101 & 0,010 & 0,0100 \\
\hline \multirow[t]{6}{*}{0,05} & W & 3 & 0,0516 & 0,0514 & 0,0511 & 0,0511 & 0,0510 & 0,0512 & 0,0516 & 0,0510 & 0,0514 \\
\hline & W & 5 & 0,0511 & 0,0501 & 0,0507 & 0,0507 & 0,0503 & 0,0509 & 0,0511 & 0,0509 & 0,0511 \\
\hline & W & 20 & 0,0501 & 0,0501 & 0,0500 & 0,0500 & 0,050 & 0,0501 & 0,05 & 0,050 & 0,0501 \\
\hline & $\mathrm{RV}$ & 3 & 0,0513 & 0,0512 & 0,0511 & 0,051 & 0,0510 & 0,0512 & 0,0511 & 0,0509 & 0,0512 \\
\hline & RV & 5 & 0,0511 & 0,0511 & 0,0507 & 0,0509 & 0,0507 & 0,0509 & 0,0509 & 0,0503 & 0,0511 \\
\hline & $\mathrm{RV}$ & 20 & 0,0501 & 0,0501 & 0,050 & 0,0500 & 0,0500 & 0,0500 & 0,05 & 0,050 & 0,0500 \\
\hline \multirow[t]{6}{*}{0,10} & W & 3 & 0,117 & 0,1083 & 0,115 & 0,111 & 0,1057 & 0,1049 & 0,1033 & 0,11 & 0,1076 \\
\hline & W & 5 & 0,108 & 0,103 & 0,109 & 0,109 & 0,101 & 0,101 & 0,101 & 0,108 & 0,104 \\
\hline & W & 20 & 0,1003 & 0,1001 & 0,1007 & 0,1007 & 0,10 & 0,10 & 0,10 & 0,1006 & 0,1001 \\
\hline & $\mathrm{RV}$ & 3 & 0,113 & 0,1071 & 0,111 & 0,1088 & 0,1071 & 0,1051 & 0,1021 & 0,11 & 0,1044 \\
\hline & $\mathrm{RV}$ & 5 & 0,108 & 0,102 & 0,109 & 0,107 & 0,103 & 0,101 & 0,101 & 0,109 & 0,101 \\
\hline & $\mathrm{RV}$ & 20 & 0,1002 & 0,10 & 0,1007 & 0,1002 & 0,10 & 0,10 & 0,10 & 0,1006 & 0,10 \\
\hline
\end{tabular}


Tabela 21: Tamanhos empíricos dos testes de Wald e Razão de Verossimilhança para a terceira hipótese com $\mathrm{p}=6$ e $\mathrm{m}=10$ - Distribuição t de Student.

\begin{tabular}{|c|c|c|c|c|c|c|c|c|c|c|c|}
\hline \multirow[b]{2}{*}{ nivel } & \multirow[b]{2}{*}{ teste } & \multirow[b]{2}{*}{ ni } & \multicolumn{3}{|c|}{$\sigma_{x_{i s}}^{2}$} & \multicolumn{3}{|c|}{$\sigma_{x}^{2}$} & \multicolumn{3}{|c|}{$\sigma_{x_{i l}}^{2}$} \\
\hline & & & $\sigma_{s}^{2}$ & $\begin{array}{r}x_{j s} \\
\sigma^{2}\end{array}$ & $\sigma_{l}^{2}$ & $\sigma_{s}^{2}$ & $\sigma_{j}^{2}$ & $\sigma_{l}^{2}$ & $\sigma_{s}^{2}$ & $\sigma^{2}$ & $\sigma_{l}^{2}$ \\
\hline \multirow[t]{6}{*}{0,01} & $\mathrm{~W}$ & 3 & 0,0112 & 0,0118 & 0,0114 & 0,0116 & 0,0119 & 0,0116 & 0,0115 & 0,0116 & 0,0116 \\
\hline & W & 5 & 0,0105 & 0,0104 & 0,0104 & 0,0109 & 0,0103 & 0,0104 & 0,0109 & 0,0101 & 0,0107 \\
\hline & W & 20 & 0,0101 & 0,0101 & 0,010 & 0,0100 & 0,010 & 0,0100 & 0,0101 & 0,0101 & 0,0100 \\
\hline & RV & 3 & 0,0115 & 0,0113 & 0,0112 & 0,0118 & 0,0113 & 0,0113 & 0,0113 & 0,0113 & 0,0113 \\
\hline & RV & 5 & 0,0109 & 0,0107 & 0,0107 & 0,0102 & 0,0107 & 0,0101 & 0,0108 & 0,0109 & 0,0105 \\
\hline & RV & 20 & 0,0102 & 0,0101 & 0,0102 & 0,0100 & 0,010 & 0,0101 & 0,010 & 0,0100 & 0,0101 \\
\hline \multirow[t]{6}{*}{0,05} & W & 3 & 0,0511 & 0,0515 & 0,0512 & 0,0514 & 0,0514 & 0,0510 & 0,0514 & 0,0515 & 0,0514 \\
\hline & W & 5 & 0,0509 & 0,0508 & 0,0504 & 0,0506 & 0,0506 & 0,0501 & 0,0509 & 0,0503 & 0,0506 \\
\hline & W & 20 & 0,0501 & 0,0500 & 0,050 & 0,0501 & 0,0500 & 0,0500 & 0,0501 & 0,050 & 0,0501 \\
\hline & RV & 3 & 0,0510 & 0,0514 & 0,0517 & 0,0515 & 0,0512 & 0,0516 & 0,0519 & 0,0511 & 0,0511 \\
\hline & RV & 5 & 0,0507 & 0,0505 & 0,0507 & 0,0502 & 0,0506 & 0,0502 & 0,0507 & 0,0502 & 0,0501 \\
\hline & RV & 20 & 0,0500 & 0,0500 & 0,0501 & 0,050 & 0,0501 & 0,050 & 0,0502 & 0,050 & 0,050 \\
\hline \multirow[t]{6}{*}{0,10} & W & 3 & 0,1193 & 0,1084 & 0,1076 & 0,1125 & 0,1121 & 0,1097 & 0,1121 & 0,1122 & 0,112 \\
\hline & W & 5 & 0,1055 & 0,1051 & 0,1044 & 0,1051 & 0,1051 & 0,1068 & 0,1059 & 0,1059 & 0,1052 \\
\hline & W & 20 & 0,101 & 0,1003 & 0,10 & 0,1005 & 0,10 & 0,1001 & 0,1009 & 0,1006 & 0,10 \\
\hline & RV & 3 & 0,1161 & 0,1054 & 0,107 & 0,117 & 0,1168 & 0,1058 & 0,1162 & 0,1184 & 0,1169 \\
\hline & RV & 5 & 0,104 & 0,102 & 0,103 & 0,103 & 0,1021 & 0,104 & 0,103 & 0,103 & 0,102 \\
\hline & RV & 20 & 0,1007 & 0,10 & 0,10 & 0,10 & 0,1005 & 0,1007 & 0,1002 & 0,10 & 0,1009 \\
\hline
\end{tabular}


Tabela 22: Tamanhos empíricos dos testes de Wald e Razão de Verossimilhança para a quarta hipótese com $\mathrm{p}=6$ e $\mathrm{m}=10$ - Distribuição t de Student.

\begin{tabular}{|c|c|c|c|c|c|c|c|c|c|c|c|}
\hline \multirow[b]{2}{*}{ nivel } & \multirow[b]{2}{*}{ teste } & \multirow[b]{2}{*}{ ni } & \multicolumn{3}{|c|}{$\sigma_{x_{i s}}^{2}$} & \multicolumn{3}{|c|}{$\sigma_{x}^{2}$} & \multicolumn{3}{|c|}{$\sigma_{x_{i l}}^{2}$} \\
\hline & & & $\sigma_{s}^{2}$ & $\begin{array}{r}x_{j s} \\
\sigma^{2}\end{array}$ & $\sigma_{l}^{2}$ & $\sigma_{s}^{2}$ & $\sigma_{j}^{2}$ & $\sigma_{l}^{2}$ & $\sigma_{s}^{2}$ & $\sigma_{j l}^{x_{j}} \sigma^{2}$ & $\sigma_{l}^{2}$ \\
\hline \multirow[t]{6}{*}{0,01} & W & 3 & 0,0113 & 0,0113 & 0,0117 & 0,0112 & 0,0119 & 0,0119 & 0,0118 & 0,0118 & 0,0113 \\
\hline & W & 5 & 0,0109 & 0,0108 & 0,0106 & 0,0109 & 0,0101 & 0,0109 & 0,0109 & 0,0108 & 0,0102 \\
\hline & W & 20 & 0,0101 & 0,0100 & 0,0102 & 0,0101 & 0,010 & 0,0102 & 0,0101 & 0,0101 & 0,0100 \\
\hline & RV & 3 & 0,0112 & 0,0112 & 0,0118 & 0,0116 & 0,0118 & 0,0112 & 0,0115 & 0,0115 & 0,0112 \\
\hline & RV & 5 & 0,0105 & 0,0105 & 0,0104 & 0,0105 & 0,0106 & 0,0106 & 0,0106 & 0,0108 & 0,0107 \\
\hline & $\mathrm{RV}$ & 20 & 0,0100 & 0,010 & 0,010 & 0,0100 & 0,0100 & 0,0102 & 0,0101 & 0,0101 & 0,010 \\
\hline \multirow[t]{6}{*}{0,05} & W & 3 & 0,0519 & 0,0511 & 0,0511 & 0,0518 & 0,0519 & 0,0518 & 0,0519 & 0,0519 & 0,0519 \\
\hline & W & 5 & 0,0507 & 0,0504 & 0,0507 & 0,0507 & 0,0502 & 0,0508 & 0,0507 & 0,0507 & 0,0507 \\
\hline & W & 20 & 0,0500 & 0,050 & 0,0501 & 0,0501 & 0,050 & 0,0500 & 0,0500 & 0,0500 & 0,0500 \\
\hline & $\mathrm{RV}$ & 3 & 0,0518 & 0,0518 & 0,0519 & 0,0512 & 0,0510 & 0,0511 & 0,0515 & 0,0518 & 0,0517 \\
\hline & $\mathrm{RV}$ & 5 & 0,0506 & 0,0506 & 0,0502 & 0,0506 & 0,0504 & 0,0509 & 0,0506 & 0,0506 & 0,0506 \\
\hline & $\mathrm{RV}$ & 20 & 0,0501 & 0,0501 & 0,05 & 0,050 & 0,0500 & 0,0500 & 0,0501 & 0,0500 & 0,0500 \\
\hline \multirow[t]{6}{*}{0,10} & W & 3 & 0,1145 & 0,1078 & 0,1064 & 0,1146 & 0,1163 & 0,1143 & 0,1155 & 0,1155 & 0,1156 \\
\hline & W & 5 & 0,103 & 0,106 & 0,103 & 0,105 & 0,103 & 0,103 & 0,102 & 0,106 & 0,103 \\
\hline & W & 20 & 0,1006 & 0,1005 & 0,10 & 0,101 & 0,1005 & 0,100 & 0,100 & 0,1008 & 0,100 \\
\hline & $\mathrm{RV}$ & 3 & 0,1134 & 0,1063 & 0,1053 & 0,1132 & 0,1152 & 0,1136 & 0,1143 & 0,1144 & 0,1147 \\
\hline & $\mathrm{RV}$ & 5 & 0,106 & 0,102 & 0,1028 & 0,101 & 0,106 & 0,102 & 0,104 & 0,108 & 0,104 \\
\hline & $\mathrm{RV}$ & 20 & 0,1005 & 0,10 & 0,10 & 0,10 & 0,1009 & 0,1002 & 0,1008 & 0,1009 & 0,1002 \\
\hline
\end{tabular}


Tabela 23: Tamanhos empíricos dos testes de Wald e Razão de Verossimilhança para a quinta hipótese com $\mathrm{p}=6$ e $\mathrm{m}=10$ - Distribuição t de Student.

\begin{tabular}{|c|c|c|c|c|c|c|c|c|c|c|c|}
\hline \multirow[b]{2}{*}{ nivel } & \multirow[b]{2}{*}{ teste } & \multirow[b]{2}{*}{ ni } & \multicolumn{3}{|c|}{$\sigma_{x_{i s}}^{2}$} & \multicolumn{3}{|c|}{$\sigma_{x}^{2}$} & \multicolumn{3}{|c|}{$\sigma_{x_{i l}}^{2}$} \\
\hline & & & $\sigma_{s}^{2}$ & $\begin{array}{r}x_{j s} \\
\sigma^{2}\end{array}$ & $\sigma_{l}^{2}$ & $\sigma_{s}^{2}$ & $\sigma_{j}^{2}$ & $\sigma_{l}^{2}$ & $\sigma_{s}^{2}$ & $\sigma_{j l}^{x_{j}} \sigma^{2}$ & $\sigma_{l}^{2}$ \\
\hline \multirow[t]{6}{*}{0,01} & $\mathrm{~W}$ & 3 & 0,0119 & 0,0112 & 0,0112 & 0,0112 & 0,0114 & 0,0113 & 0,0113 & 0,0118 & 0,0118 \\
\hline & W & 5 & 0,0109 & 0,0107 & 0,0107 & 0,0106 & 0,0108 & 0,0105 & 0,0108 & 0,0106 & 0,0104 \\
\hline & W & 20 & 0,0102 & 0,0102 & 0,0101 & 0,0101 & 0,010 & 0,0100 & 0,0102 & 0,010 & 0,010 \\
\hline & RV & 3 & 0,0115 & 0,0118 & 0,0118 & 0,0119 & 0,0118 & 0,0112 & 0,0114 & 0,0112 & 0,0119 \\
\hline & RV & 5 & 0,0107 & 0,0107 & 0,0106 & 0,0103 & 0,0105 & 0,0104 & 0,0105 & 0,0104 & 0,0101 \\
\hline & RV & 20 & 0,0103 & 0,0100 & 0,0101 & 0,010 & 0,0102 & 0,0100 & 0,0100 & 0,010 & 0,010 \\
\hline \multirow[t]{6}{*}{0,05} & W & 3 & 0,0517 & 0,0518 & 0,0511 & 0,0514 & 0,0512 & 0,0518 & 0,0519 & 0,0519 & 0,0512 \\
\hline & W & 5 & 0,0507 & 0,0502 & 0,0502 & 0,0503 & 0,0504 & 0,0506 & 0,0508 & 0,0506 & 0,0501 \\
\hline & W & 20 & 0,0501 & 0,050 & 0,050 & 0,0500 & 0,0501 & 0,0501 & 0,0500 & 0,050 & 0,050 \\
\hline & RV & 3 & 0,0518 & 0,0518 & 0,0517 & 0,0517 & 0,0517 & 0,0518 & 0,0518 & 0,0519 & 0,0519 \\
\hline & RV & 5 & 0,0508 & 0,0508 & 0,0506 & 0,0508 & 0,0501 & 0,0501 & 0,0507 & 0,0502 & 0,0508 \\
\hline & RV & 20 & 0,0501 & 0,0502 & 0,0500 & 0,0500 & 0,050 & 0,050 & 0,0500 & 0,050 & 0,0501 \\
\hline \multirow[t]{6}{*}{0,10} & W & 3 & 0,1137 & 0,1145 & 0,1129 & 0,1134 & 0,1149 & 0,1148 & 0,1163 & 0,115 & 0,113 \\
\hline & W & 5 & 0,1038 & 0,1042 & 0,1076 & 0,102 & 0,1034 & 0,1062 & 0,1082 & 0,102 & 0,1026 \\
\hline & W & 20 & 0,1002 & 0,1009 & 0,102 & 0,10 & 0,10 & 0,1001 & 0,1003 & 0,10 & 0,10 \\
\hline & RV & 3 & 0,1134 & 0,1135 & 0,1124 & 0,1128 & 0,1143 & 0,1141 & 0,1154 & 0,1141 & 0,1136 \\
\hline & RV & 5 & 0,1022 & 0,1013 & 0,1016 & 0,1066 & 0,103 & 0,1036 & 0,102 & 0,101 & 0,1023 \\
\hline & RV & 20 & 0,10 & 0,1003 & 0,10 & 0,101 & 0,1007 & 0,1009 & 0,10 & 0,10 & 0,1002 \\
\hline
\end{tabular}


Tabela 24: Tamanhos empíricos dos testes de Wald e Razão de Verossimilhança para a sexta hipótese com $\mathrm{p}=6$ e $\mathrm{m}=10$ - Distribuição t de Student.

\begin{tabular}{|c|c|c|c|c|c|c|c|c|c|c|c|}
\hline \multirow[b]{2}{*}{ nivel } & \multirow[b]{2}{*}{ teste } & \multirow[b]{2}{*}{ ni } & \multicolumn{3}{|c|}{$\sigma_{x_{j s}}^{2}$} & \multicolumn{3}{|c|}{$\sigma_{x_{j}}^{2}$} & \multicolumn{3}{|c|}{$\sigma_{x_{j l}}^{2}$} \\
\hline & & & $\sigma_{s}^{2}$ & $\sigma^{2}$ & $\sigma_{l}^{2}$ & $\sigma_{s}^{2}$ & $\sigma^{2}$ & $\sigma_{l}^{2}$ & $\sigma_{s}^{2}$ & $\sigma^{2}$ & $\sigma_{l}^{2}$ \\
\hline \multirow[t]{6}{*}{0,01} & $\mathrm{~W}$ & 3 & 0,0110 & 0,0115 & 0,0115 & 0,0119 & 0,0112 & 0,0118 & 0,0116 & 0,0117 & 0,0113 \\
\hline & W & 5 & 0,0101 & 0,0104 & 0,0103 & 0,0104 & 0,0105 & 0,0106 & 0,0109 & 0,0108 & 0,0108 \\
\hline & W & 20 & 0,010 & 0,0100 & 0,010 & 0,0100 & 0,010 & 0,0102 & 0,0103 & 0,0101 & 0,0103 \\
\hline & RV & 3 & 0,0119 & 0,0115 & 0,0114 & 0,0118 & 0,0112 & 0,0116 & 0,0118 & 0,0115 & 0,1122 \\
\hline & RV & 5 & 0,0104 & 0,0104 & 0,0102 & 0,0103 & 0,0104 & 0,0105 & 0,0108 & 0,0107 & 0,0107 \\
\hline & RV & 20 & 0,0100 & 0,01 & 0,01 & 0,01 & 0,0100 & 0,0102 & 0,0103 & 0,0101 & 0,0103 \\
\hline \multirow[t]{6}{*}{0,05} & W & 3 & 0,0516 & 0,0517 & 0,0511 & 0,0519 & 0,0515 & 0,0512 & 0,0518 & 0,0516 & 0,0516 \\
\hline & W & 5 & 0,0505 & 0,0506 & 0,0506 & 0,0506 & 0,0502 & 0,0504 & 0,0506 & 0,0502 & 0,0508 \\
\hline & W & 20 & 0,0501 & 0,0500 & 0,0500 & 0,0500 & 0,050 & 0,050 & 0,0500 & 0,050 & 0,0500 \\
\hline & RV & 3 & 0,0514 & 0,0516 & 0,0510 & 0,0516 & 0,0517 & 0,0517 & 0,0513 & 0,0517 & 0,0518 \\
\hline & RV & 5 & 0,0504 & 0,0502 & 0,0502 & 0,0506 & 0,0509 & 0,0507 & 0,0506 & 0,0504 & 0,0502 \\
\hline & RV & 20 & 0,0500 & 0,050 & 0,050 & 0,0500 & 0,0500 & 0,0500 & 0,0500 & 0,0501 & 0,0500 \\
\hline \multirow[t]{6}{*}{0,10} & W & 3 & 0,1131 & 0,1121 & 0,1114 & 0,1145 & 0,1135 & 0,1125 & 0,112 & 0,1122 & 0,1146 \\
\hline & W & 5 & 0,1094 & 0,1011 & 0,1017 & 0,1022 & 0,1042 & 0,1026 & 0,106 & 0,108 & 0,1094 \\
\hline & W & 20 & 0,101 & 0,10 & 0,10 & 0,1008 & 0,1002 & 0,10 & 0,1003 & 0,1002 & 0,1002 \\
\hline & RV & 3 & 0,1129 & 0,1121 & 0,113 & 0,1122 & 0,1125 & 0,1123 & 0,113 & 0,119 & 0,1142 \\
\hline & RV & 5 & 0,1092 & 0,102 & 0,106 & 0,1019 & 0,102 & 0,108 & 0,102 & 0,109 & 0,1017 \\
\hline & RV & 20 & 0,103 & 0,10 & 0,1003 & 0,10 & 0,1008 & 0,102 & 0,10 & 0,1003 & 0,10 \\
\hline
\end{tabular}

Depois de analisar as tabelas apresentadas nesta Seção e no Apêndice B, podemos notar que em todos os casos conforme o número de réplicas aumenta, o valor empírico se aproxima do valor nominal. Comparando as tabelas aqui apresentadas e as do Apêndice B, ou seja, mudando o número de laboratórios (p) e o número de patamares (m), os resultados são muito parecidos, indicando que o p e o m não influenciam nos resultados. Notamos também que para os testes Wald e Razão de Verossimilhança, quando variamos $\sigma_{x}^{2}$ e $\sigma^{2}$, concluímos que valores dos parâmetros $\sigma_{x}^{2}$ e $\sigma^{2}$ não influenciam no resultado, visto que as mudanças foram muito pequenas e não ocorreram em todos os níveis. 


\subsection{Resultados da simulação para o poder do teste: distribui- ções Normal e t de Student}

Nesta Seção apresentaremos os gráficos do poder do teste para as duas distribuições, com 5 réplicas, 6 laboratórios e 10 rotações (ou seja, valores usados na aplicação). Os demais gráficos podem ser encontrados no Apêndice C.

A seguir, apresentamos os gráficos para a distribuição Normal: 
Hip1-F- P.T. Wald

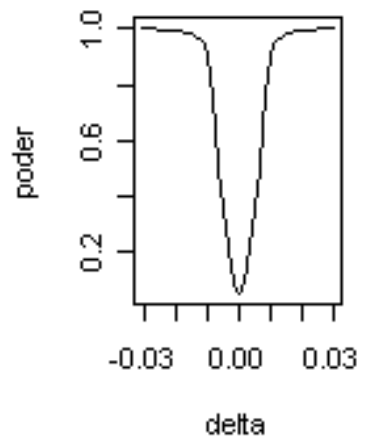

Hip3-F- P.T. Wald

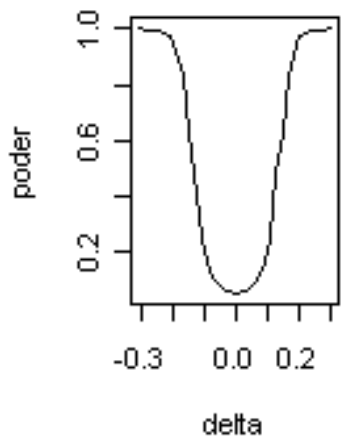

Hip5-F- P.T. Wald

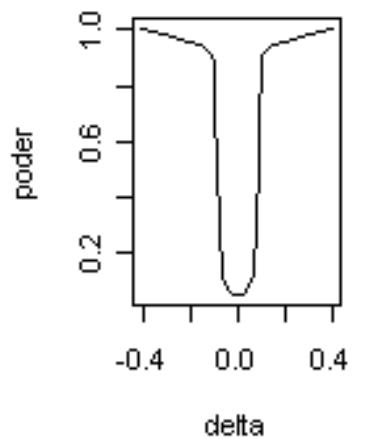

Hip1-F- P.T. RV

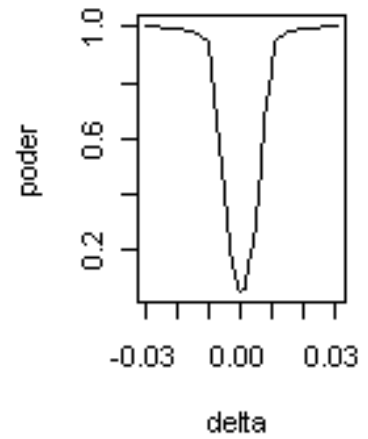

Hip3-F- P.T. RV

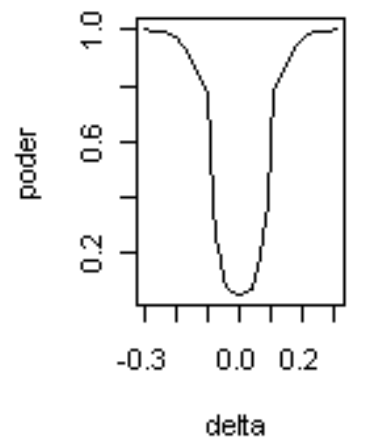

Hip5-F- P.T. RV

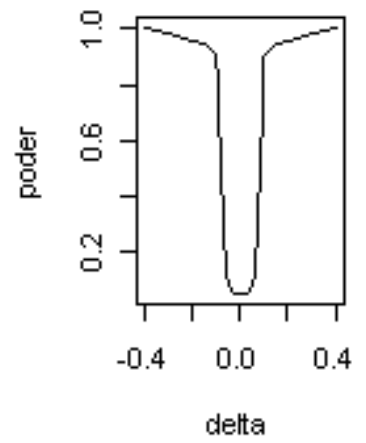

Hip2-F- P.T. Wald

Hip2-F- P.T. RV

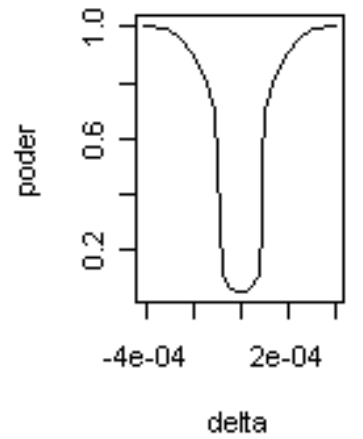

Hip4-F- P.T. Wald

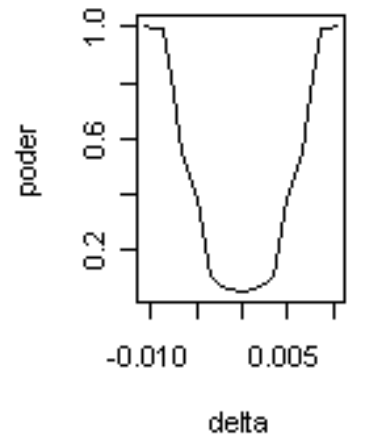

Hip6-F- P.T. Wald

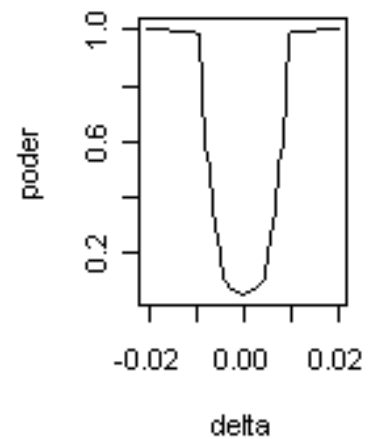

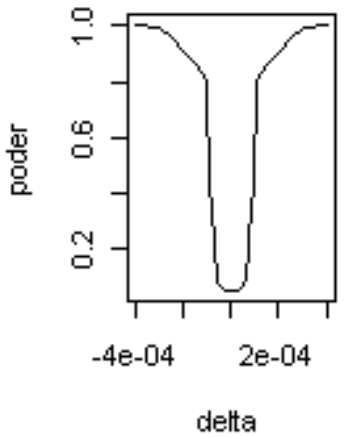

Hip4-F- P.T. RV

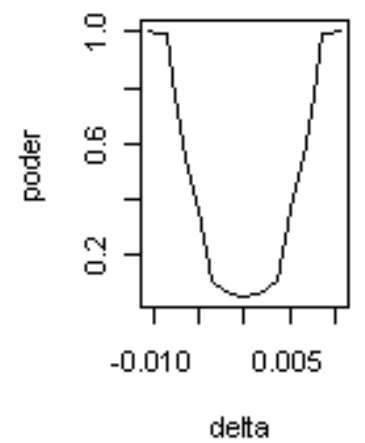

Hip6-F- P.T. RV

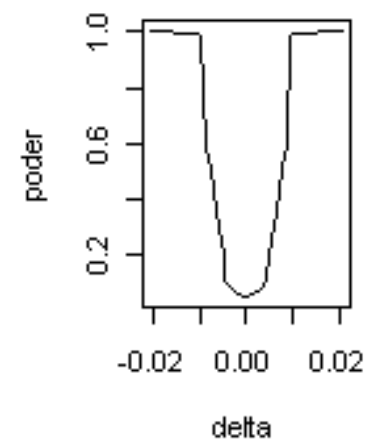

Figura 1: Gráficos do P. T. de Wald e RV para 5 réplicas- 6 laboratórios e 10 rotações $\left(\sigma_{x j s}^{2} \operatorname{com} \sigma_{s}^{2}\right)$. 
Hip1-G-P.T. Wald

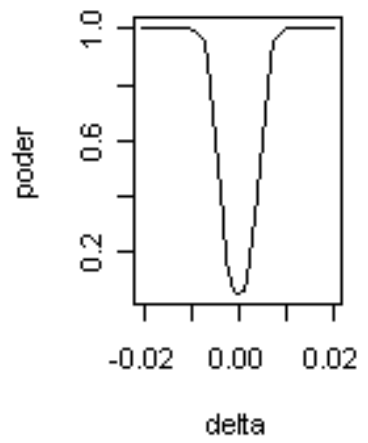

Hip3-G- P.T. Wald

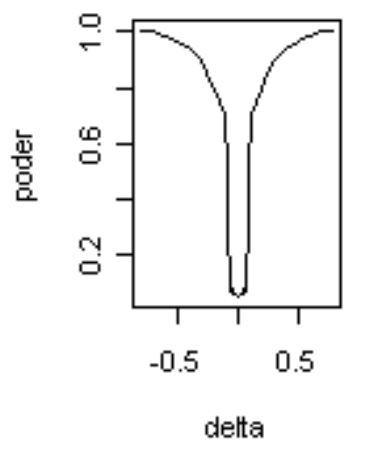

Hip5-G- P.T. Wald

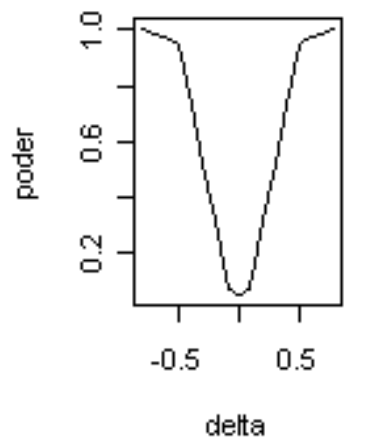

Hip1-G- P.T. RV

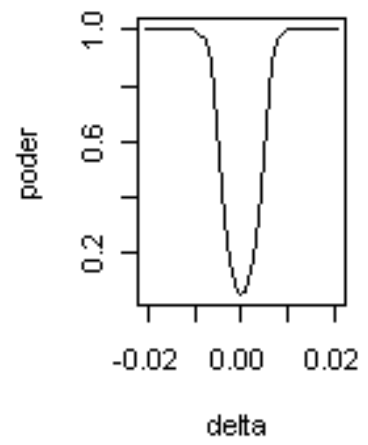

Hip3-G- P.T. RV

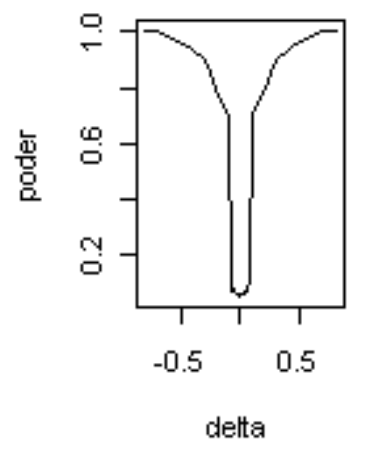

Hip5-G- P.T. RV

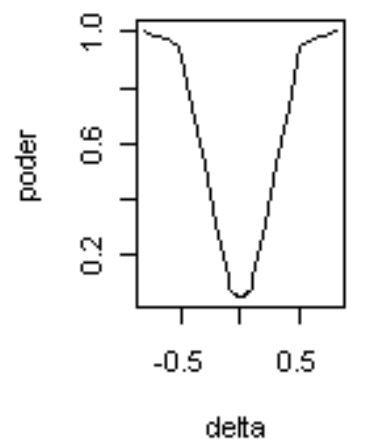

Hip2-G- P.T. Wald

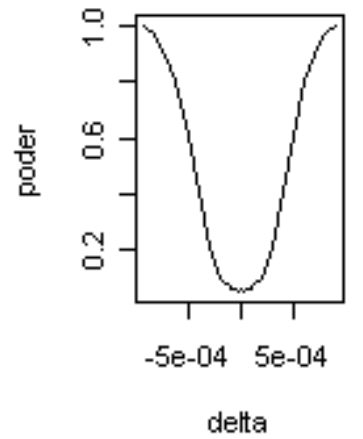

Hip4-G- P.T. Wald

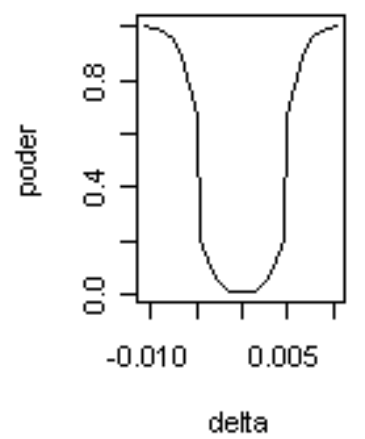

Hip6-G- P.T. Wald

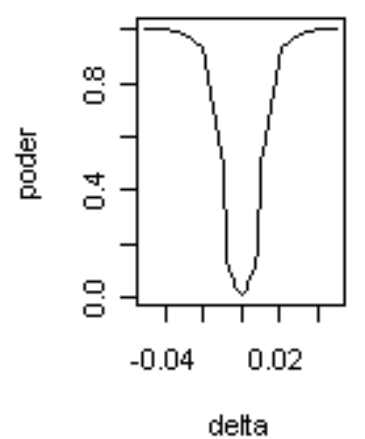

Hip2-G- P.T. RV

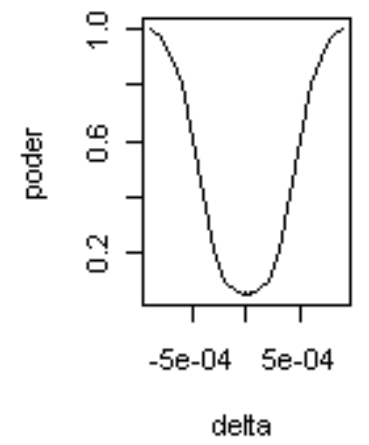

Hip4-G- P.T. RV

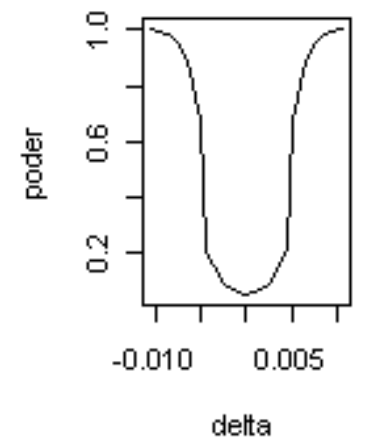

Hip6-G- P.T. RV

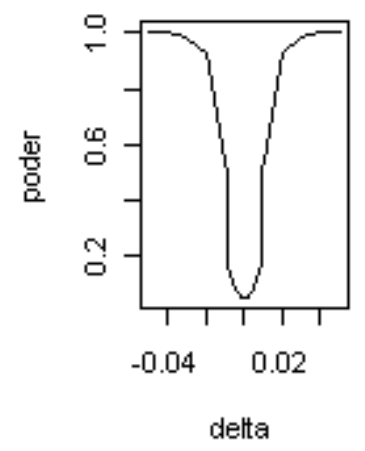

Figura 2: Gráficos do P. T. de Wald e RV para 5 réplicas- 6 laboratórios e 10 rotações $\left(\sigma_{x j s}^{2} \operatorname{com} \sigma^{2}\right)$. 
Hip1-B-P.T. Wald

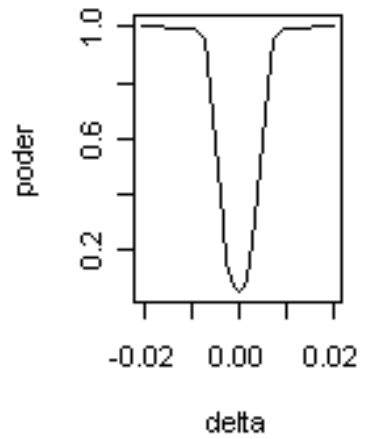

Hip3-B-P.T. Wald

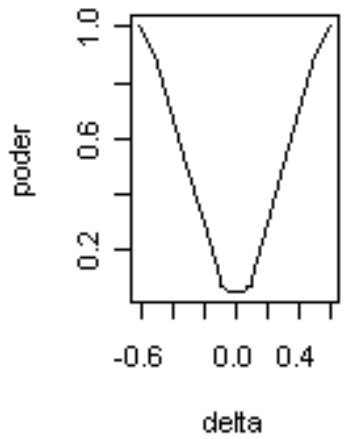

Hip5-B- P.T. Wald

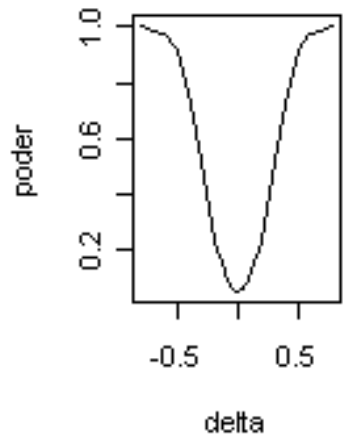

Hip1-B- P.T. RV

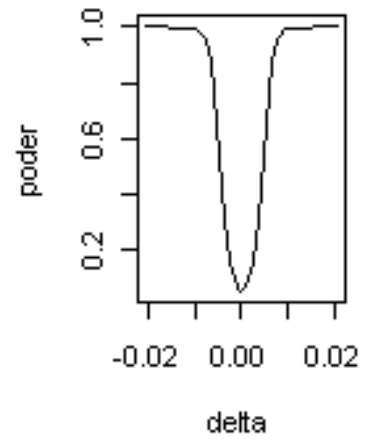

Hip3-B- P.T. RV

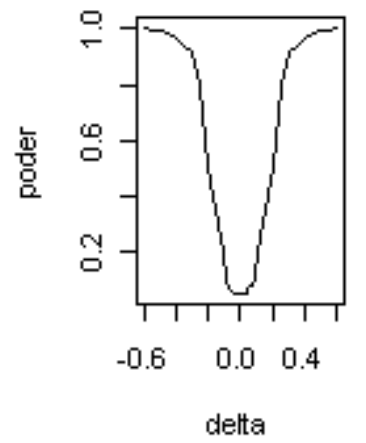

Hip5-B- P.T. RV

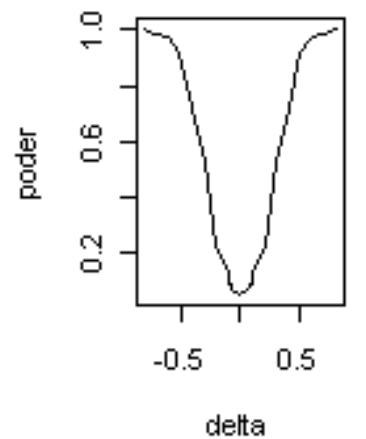

Hip2-B- P.T. Wald

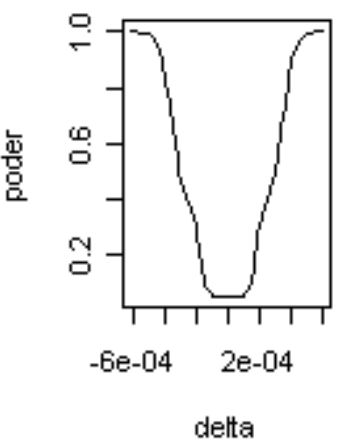

Hip4-B- P.T. Wald

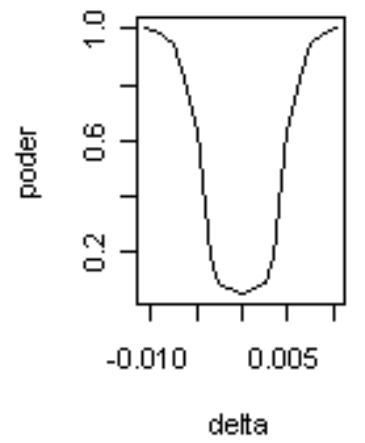

Hip6-B-P.T. Wald

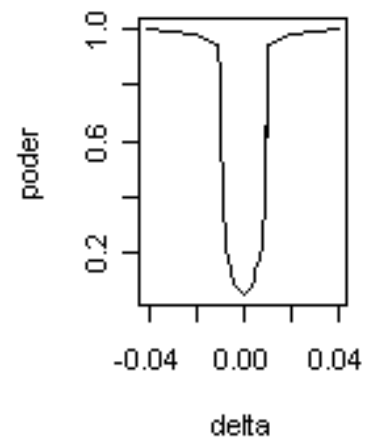

Hip2-B- P.T. RV

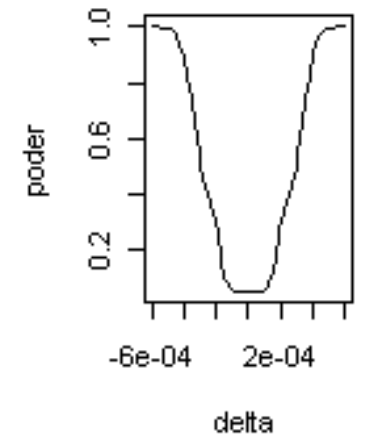

Hip4-B- P.T. RV

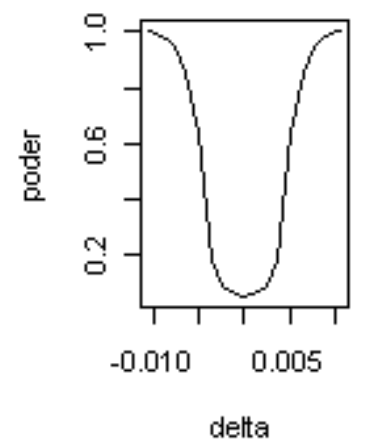

Hip6-B- P.T. RV

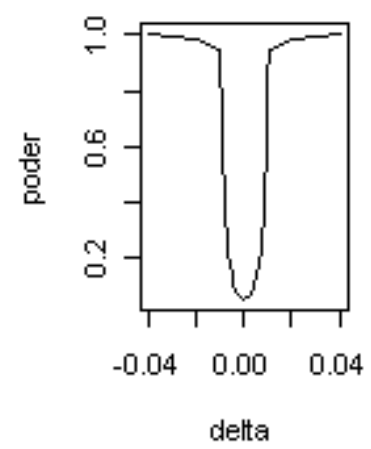

Figura 3: Gráficos do P. T. de Wald e RV para 5 réplicas- 6 laboratórios e 10 rotações $\left(\sigma_{x j s}^{2} \operatorname{com} \sigma_{l}^{2}\right)$. 
Hip1-D-P.T. Wald

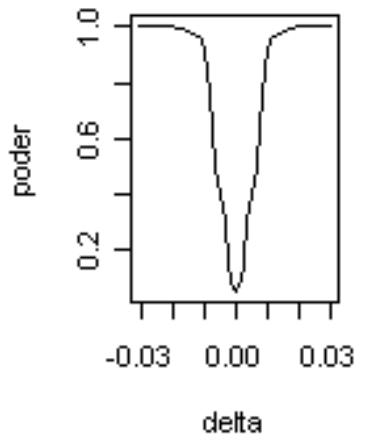

Hip3-D- P.T. Wald

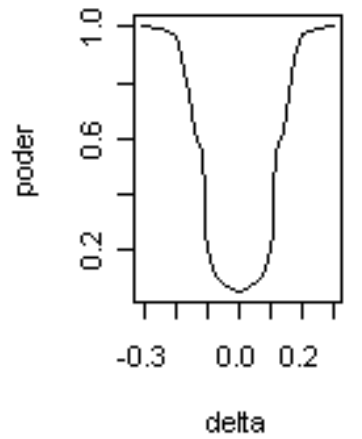

Hip5-D- P.T. Wald

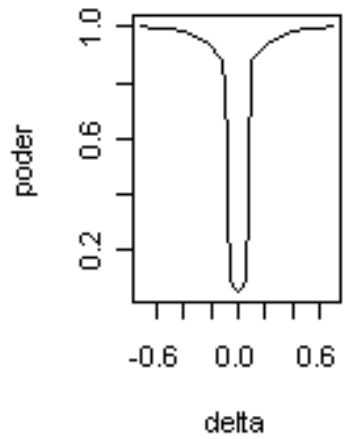

Hip1-D- P.T. RV

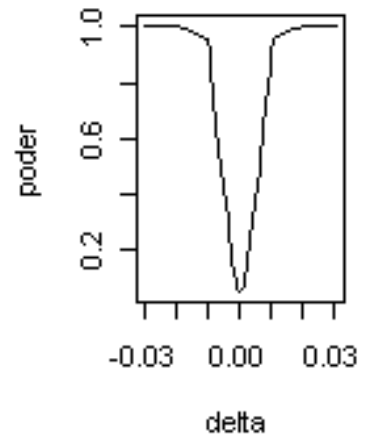

Hip3-D- P.T. RV

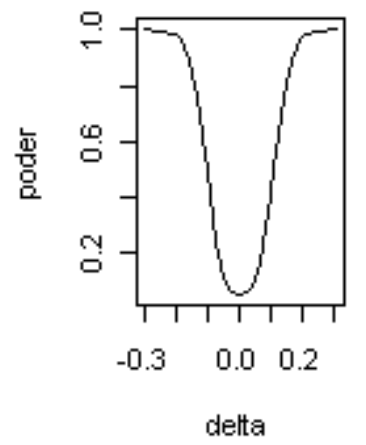

Hip5-D- P.T. RV

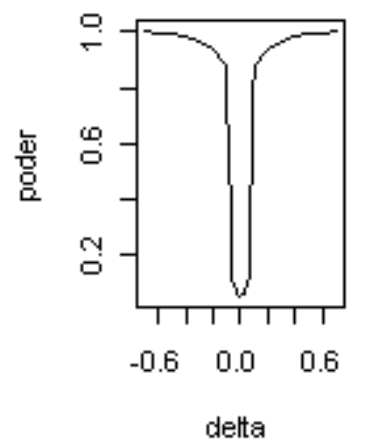

Hip2-D- P.T. Wald

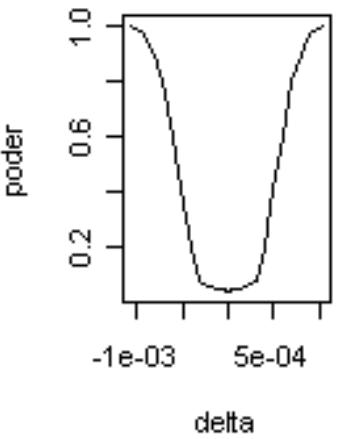

Hip4-D- P.T. Wald

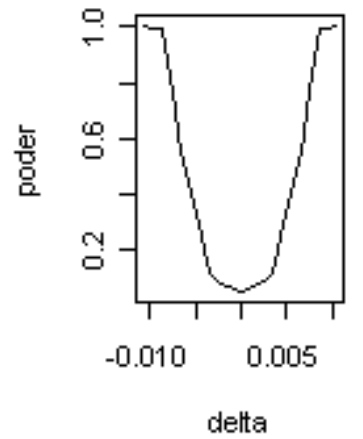

Hip6-D- P.T. Wald

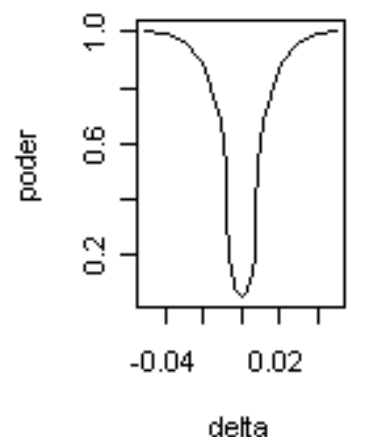

Hip2-D- P.T. RV

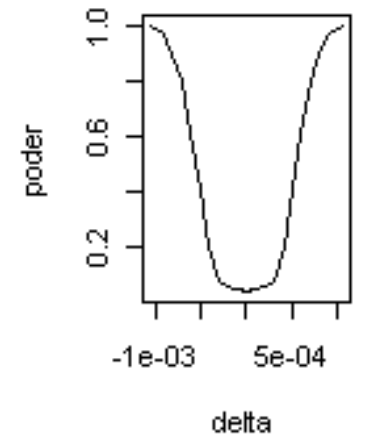

Hip4-D- P.T. RV

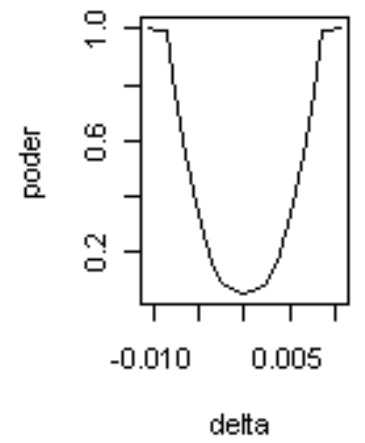

Hip6-D- P.T. RV

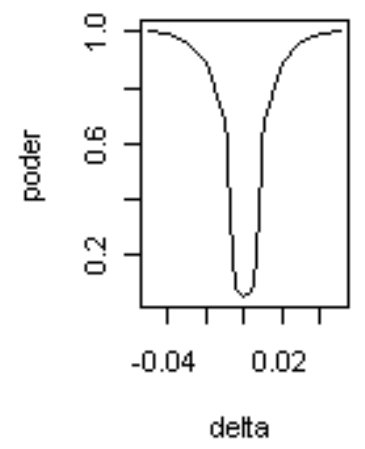

Figura 4: Gráficos do P. T. de Wald e RV para 5 réplicas- 6 laboratórios e 10 rotações $\left(\sigma_{x j}^{2} \operatorname{com} \sigma_{s}^{2}\right)$. 
Hip1-A- P.T. Wald

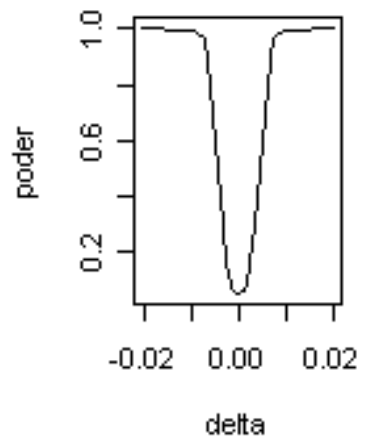

Hip3-A- P.T. Wald

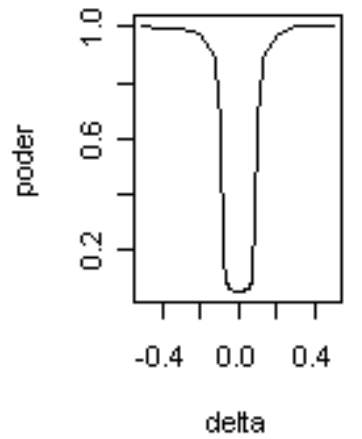

Hip5-A- P.T. Wald

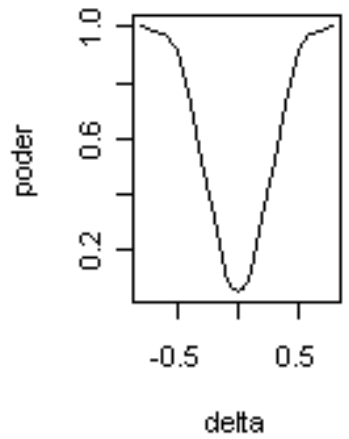

Hip1-A- P.T. RV

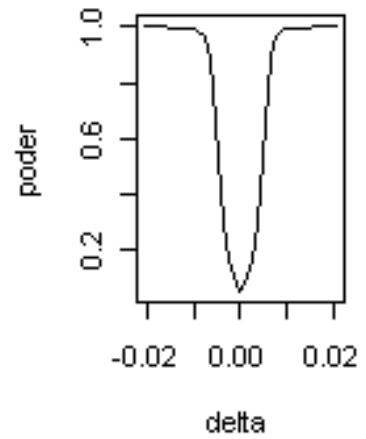

Hip3-A- P.T. RV

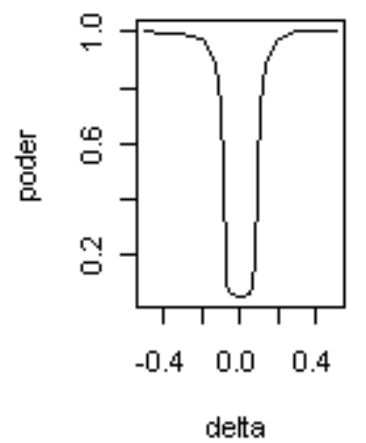

Hip5-A- P.T. RV

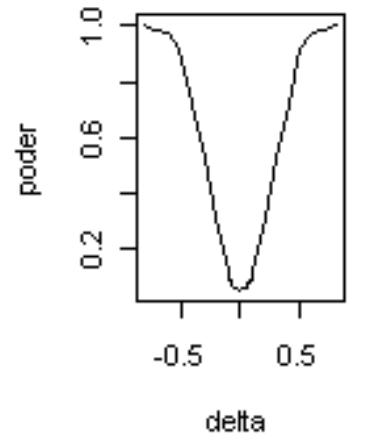

Hip2-A- P.T. Wald

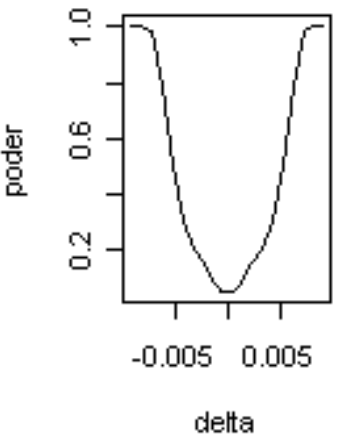

Hip4-A- P.T. Wald

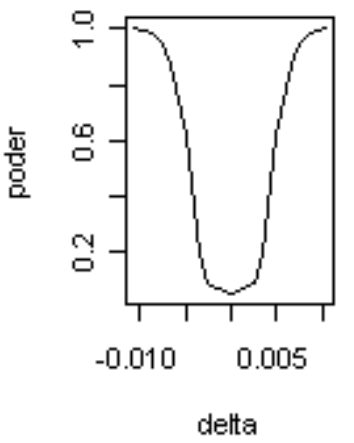

Hip6-A- P.T. Wald

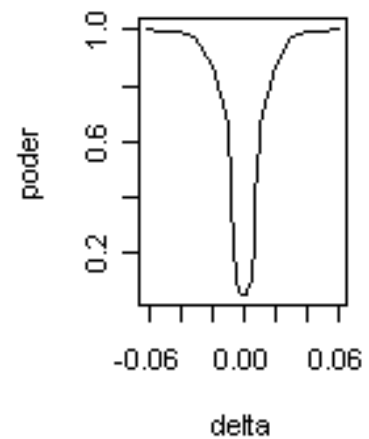

Hip2-A- P.T. RV

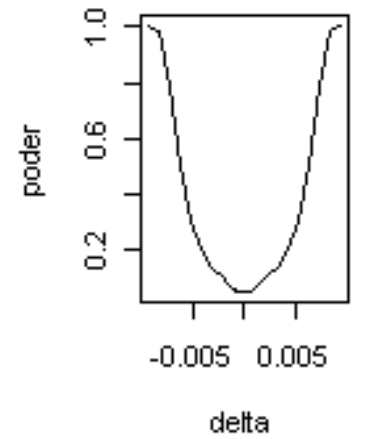

Hip4-A- P.T. RV

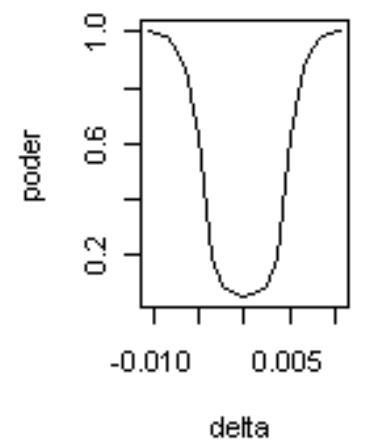

Hip6-A- P.T. RV

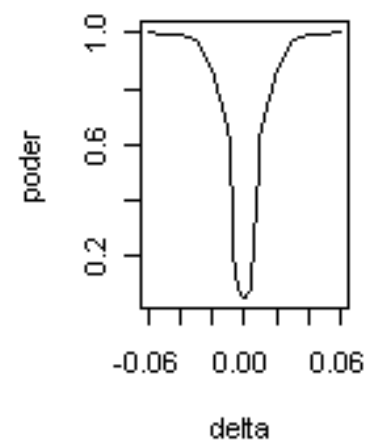

Figura 5: Gráficos do P. T. de Wald e RV para 5 réplicas- 6 laboratórios e 10 rotações $\left(\sigma_{x j}^{2} \operatorname{com} \sigma^{2}\right)$. 
Hip1-E- P.T. Wald

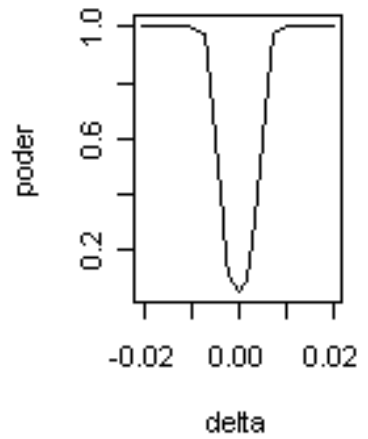

Hip3-E- P.T. Wald

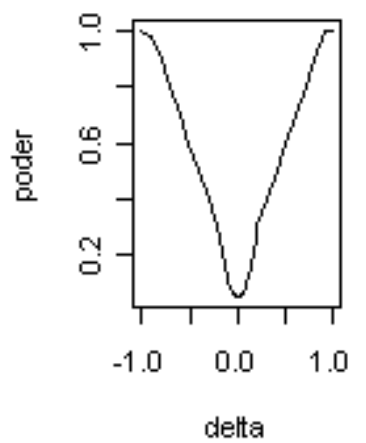

Hip5-E- P.T. Wald

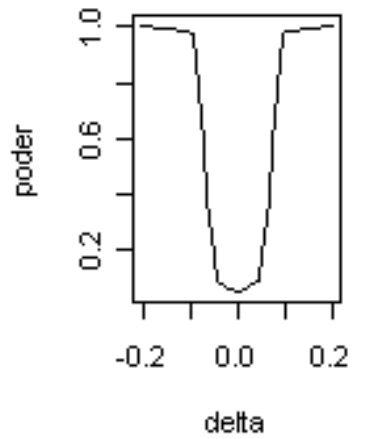

Hip1-E- P.T. RV

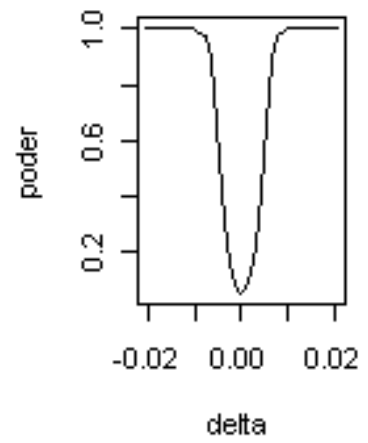

Hip3-E- P.T. RV

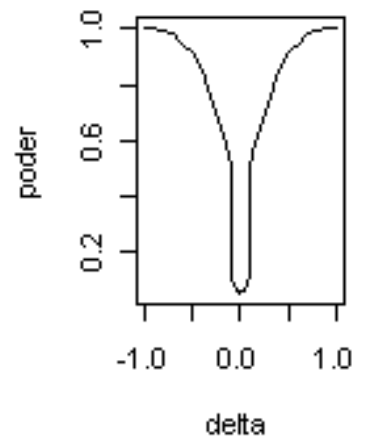

Hip5-E- P.T. RV

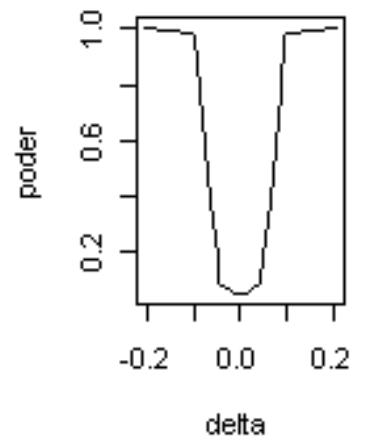

Hip2-E- P.T. Wald

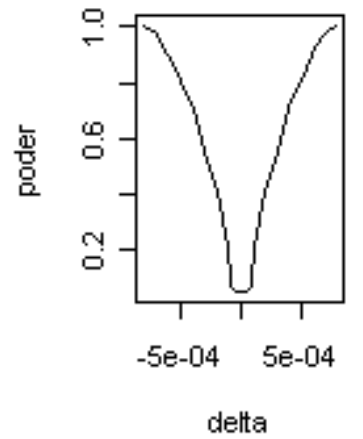

Hip4-E- P.T. Wald

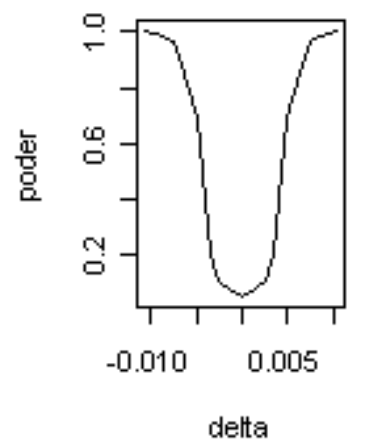

Hip6-E- P.T. Wald

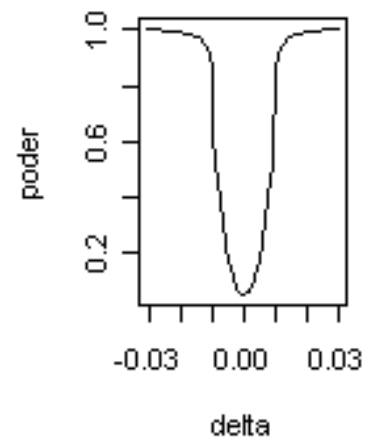

Hip2-E- P.T. RV

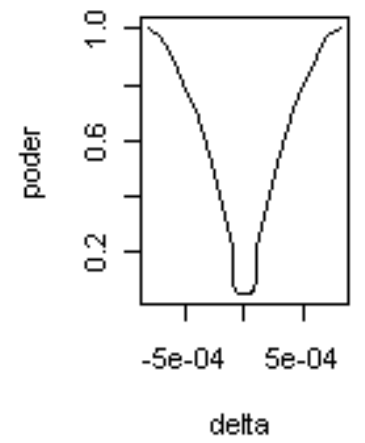

Hip4-E- P.T. RV

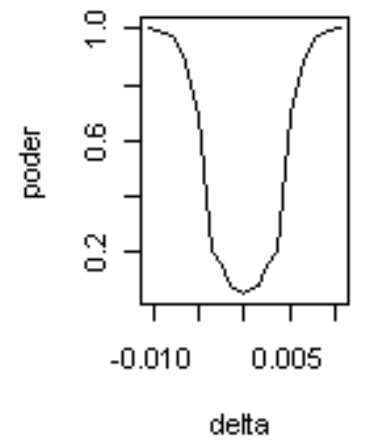

Hip6-E- P.T. RV

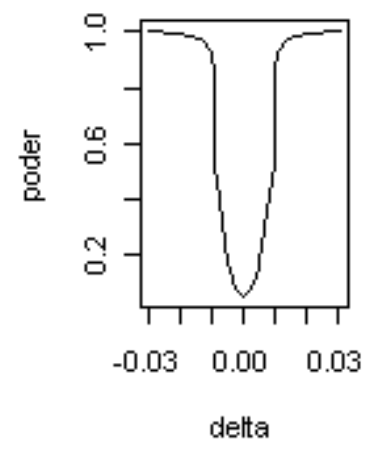

Figura 6: Gráficos do P. T. de Wald e RV para 5 réplicas- 6 laboratórios e 10 rotações $\left(\sigma_{x j}^{2} \operatorname{com} \sigma_{l}^{2}\right)$. 
Hip1-H- P.T. Wald

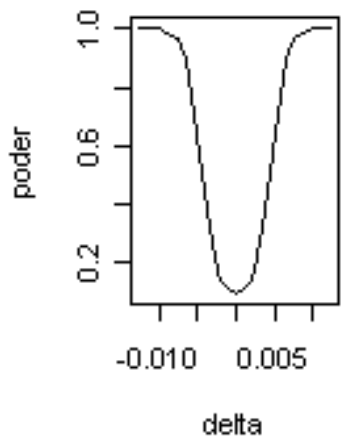

Hip3-H- P.T. Wald

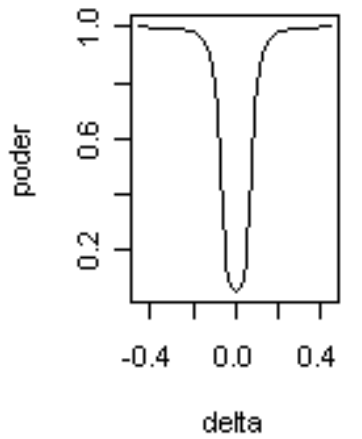

Hip5-H- P.T. Wald

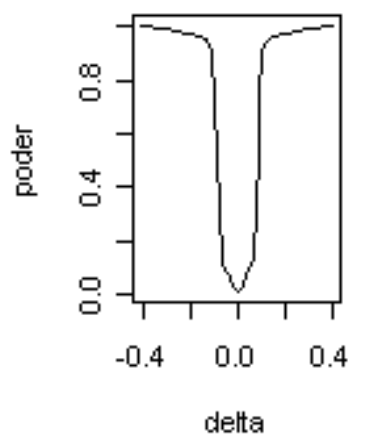

Hip1-H- P.T. RV

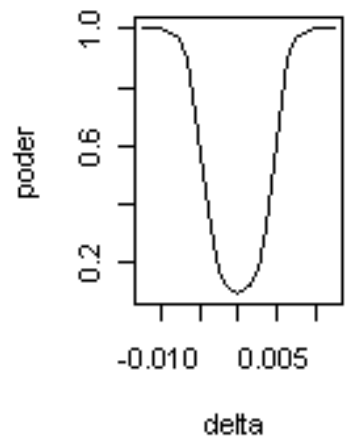

Hip3-H- P.T. RV

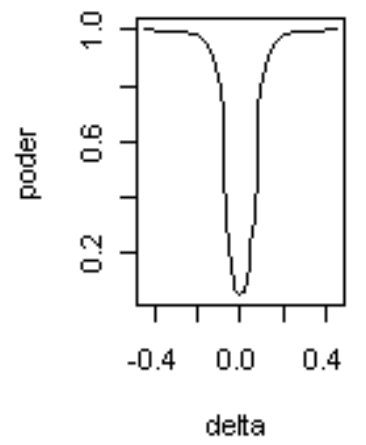

Hip5-H- P.T. RV

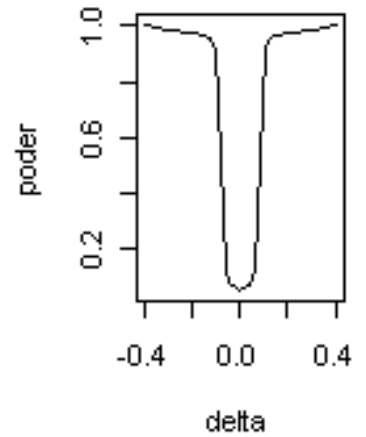

Hip2-H- P.T. Wald

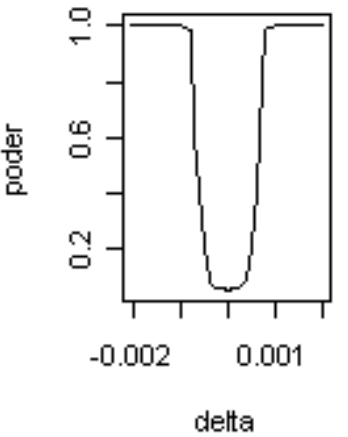

Hip4-H- P.T. Wald

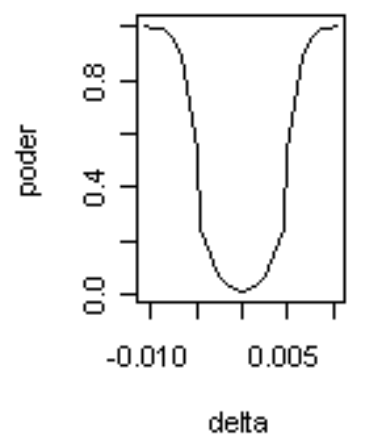

Hip6-H- P.T. Wald

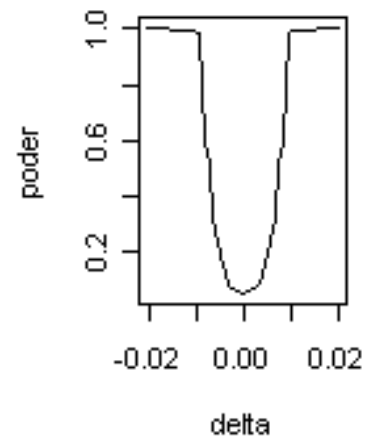

Hip2-H- P.T. RV

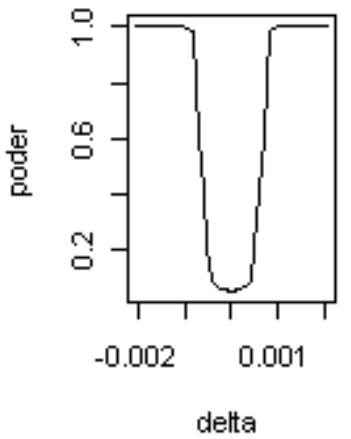

Hip4-H- P.T. RV

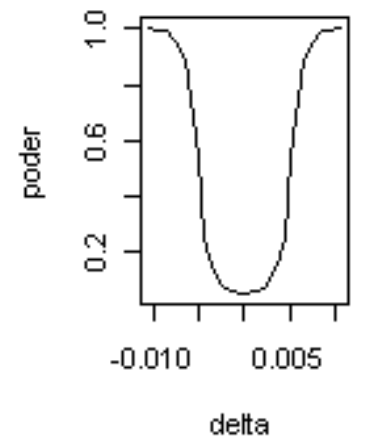

Hip6-H- P.T. RV

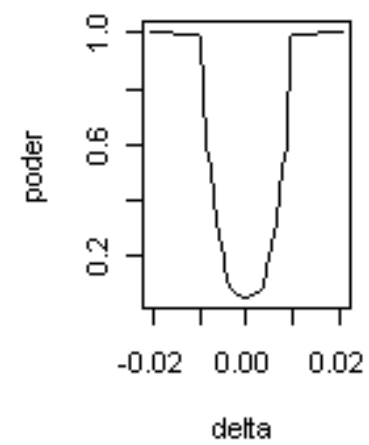

Figura 7: Gráficos do P. T. de Wald e RV para 5 réplicas- 6 laboratórios e 10 rotações $\left(\sigma_{x j l}^{2} \operatorname{com} \sigma_{s}^{2}\right)$. 
Hip1-C- P.T. Wald

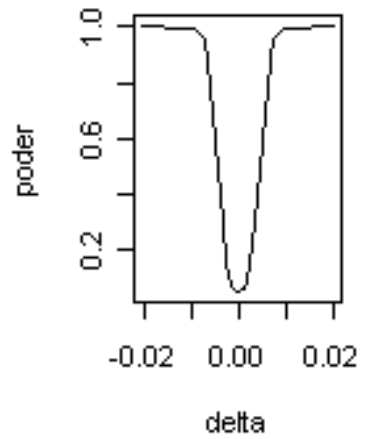

Hip3-C- P.T. Wald

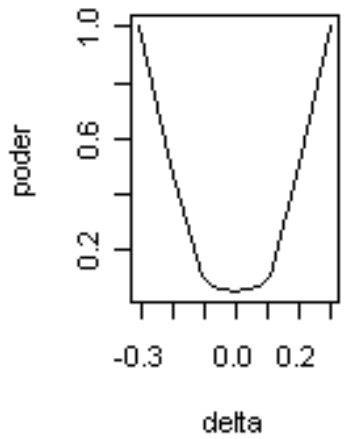

Hip5-C- P.T. Wald

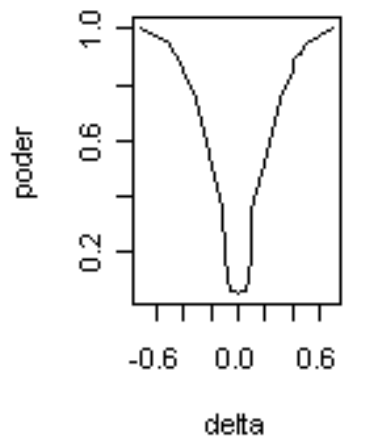

Hip1-C- P.T. RV

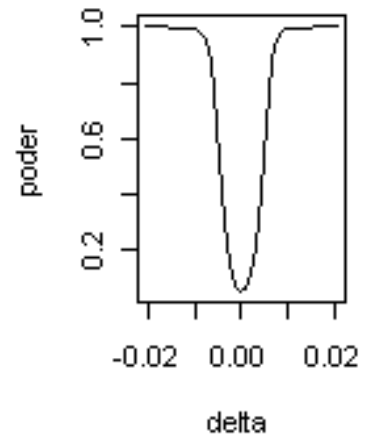

Hip3-C- P.T. RV

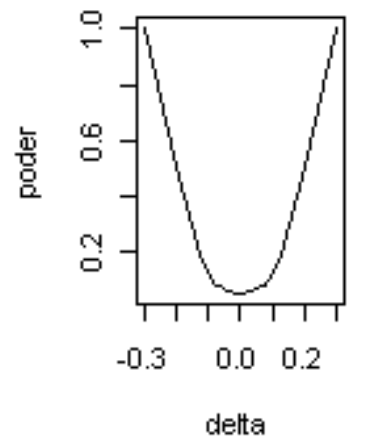

Hip5-C- P.T. RV

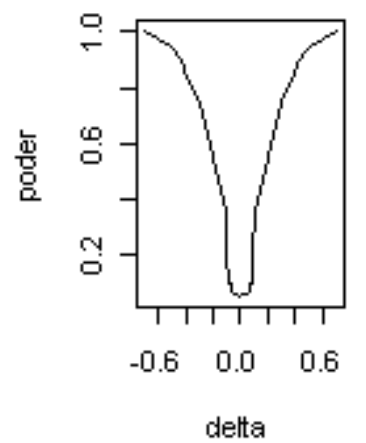

Hip2-C- P.T. Wald

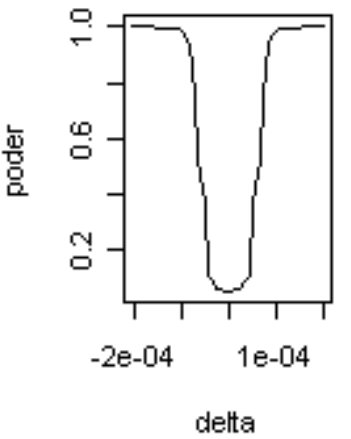

Hip4-C- P.T. Wald

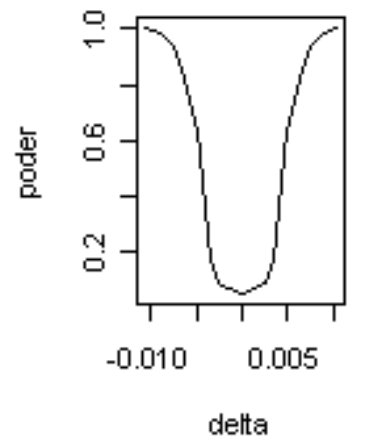

Hip6-C- P.T. Wald

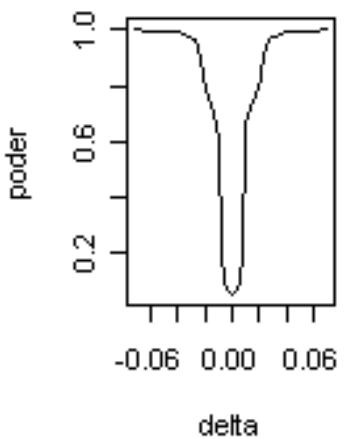

Hip2-C- P.T. RV

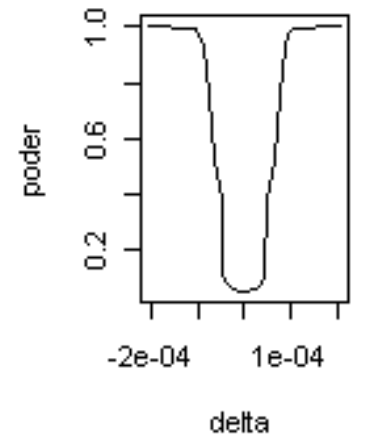

Hip4-C- P.T. RV

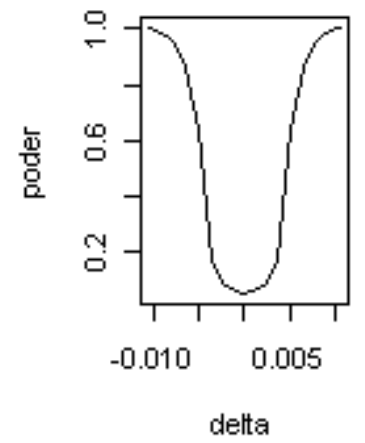

Hip6-C- P.T. RV

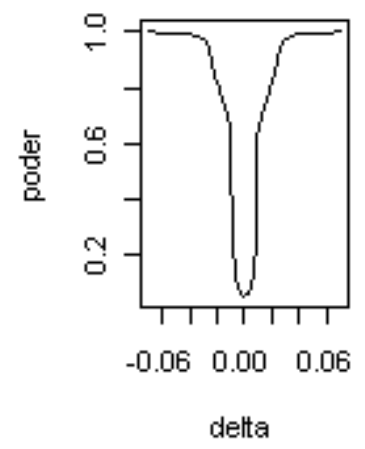

Figura 8: Gráficos do P. T. de Wald e RV para 5 réplicas- 6 laboratórios e 10 rotações $\left(\sigma_{x j l}^{2} \operatorname{com} \sigma^{2}\right)$. 
Hip1-I- P.T. Wald

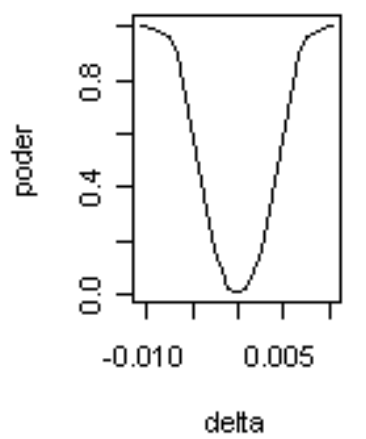

Hip3-I- P.T. Wald

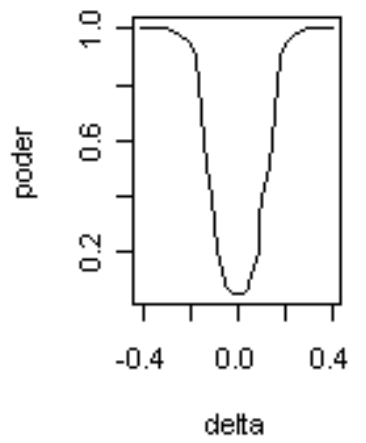

Hip5-I- P.T. Wald

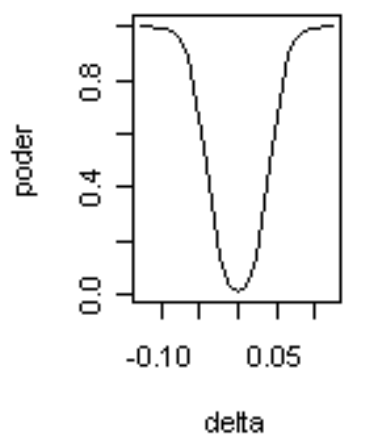

Hip1-I- P.T. RV

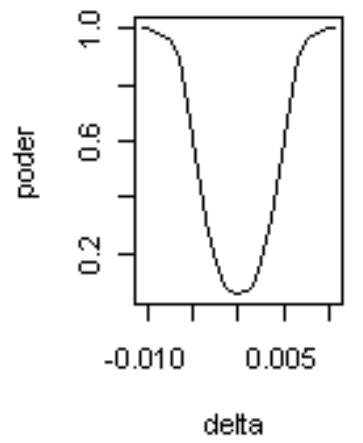

Hip3-I- P.T. RV

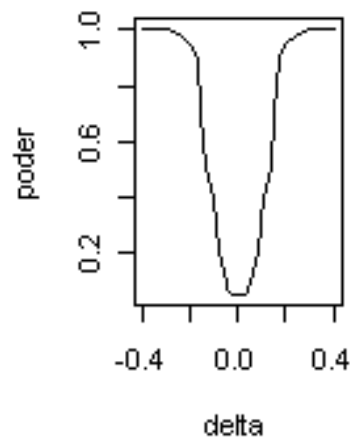

Hip5-I- P.T. RV

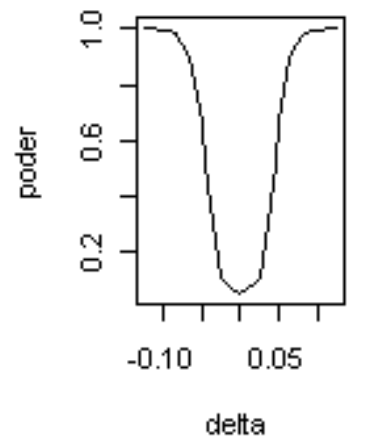

Hip2-I- P.T. Wald

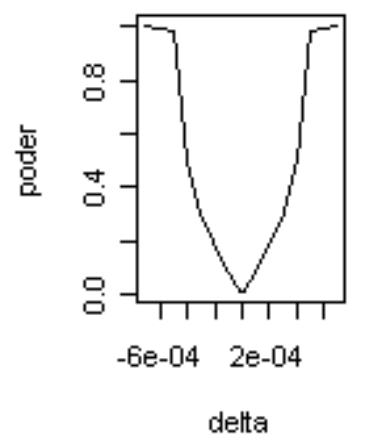

Hip4-I- P.T. Wald

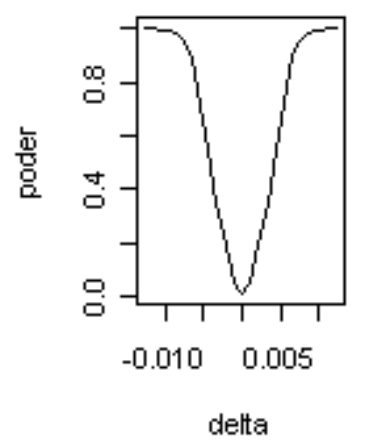

Hip6-I- P.T. Wald

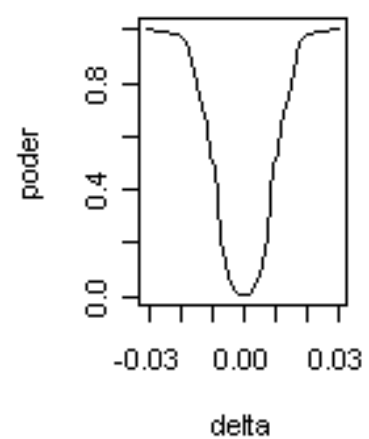

Hip2-I- P.T. RV

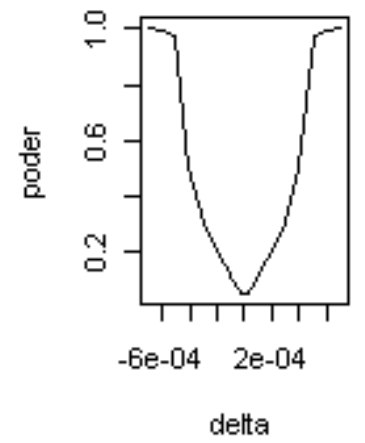

Hip4-I- P.T. RV

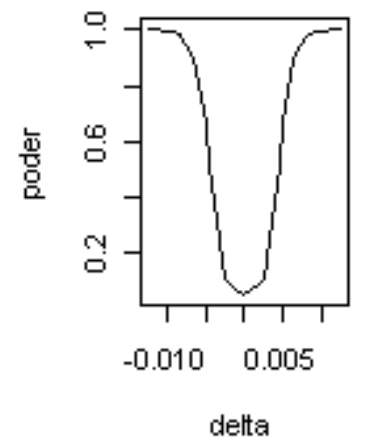

Hip6-I- P.T. RV

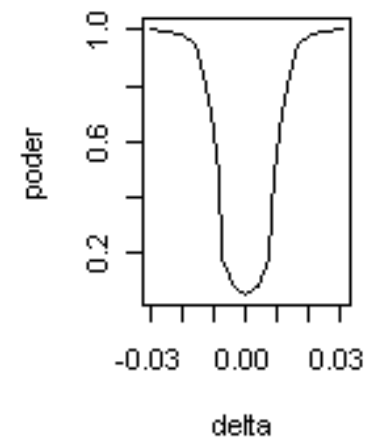

Figura 9: Gráficos do P. T. de Wald e RV para 5 réplicas- 6 laboratórios e 10 rotações $\left(\sigma_{x j l}^{2} \operatorname{com} \sigma_{l}^{2}\right)$.

A seguir, os gráficos da distribuição t de Student: 
Hip1- F. P. T. Wald

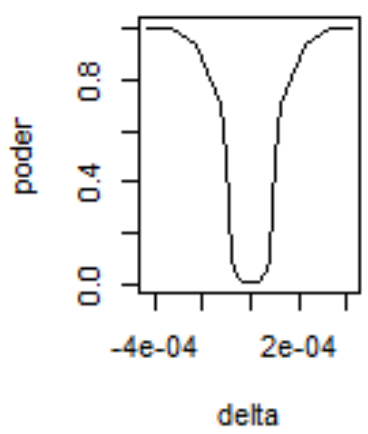

Hip3- F- P. T. Wald

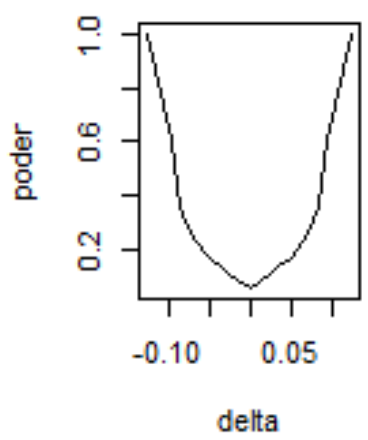

Hip5- F- P. T. Wald

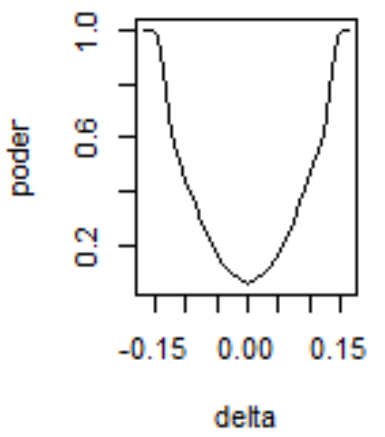

Hip1- F- P. T. RV

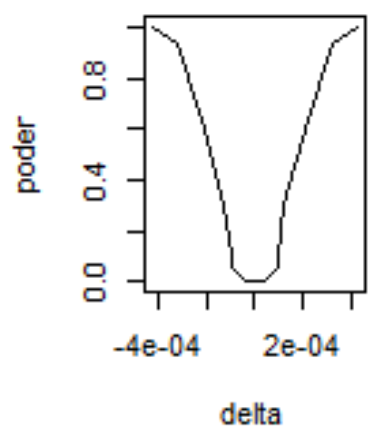

Hip3- F- P. T. RV

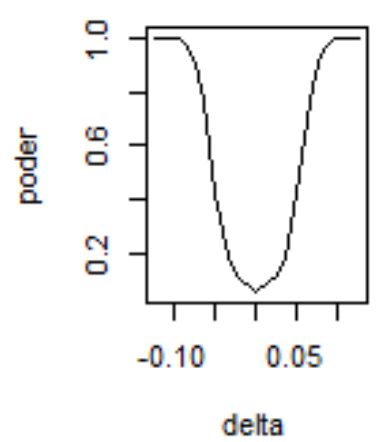

Hip5- F- P. T. RV

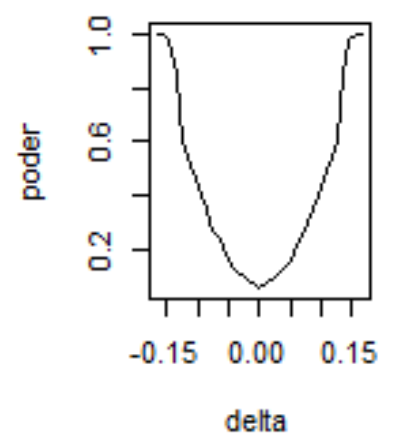

Hip2- F- P. T. Wald

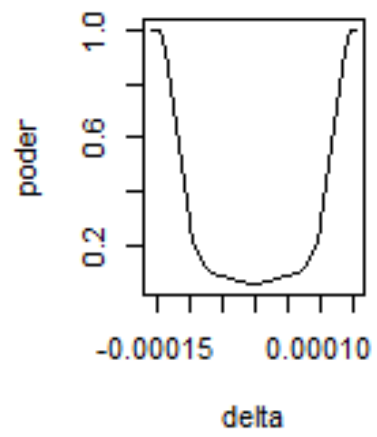

Hip4- F- P. T. Wald

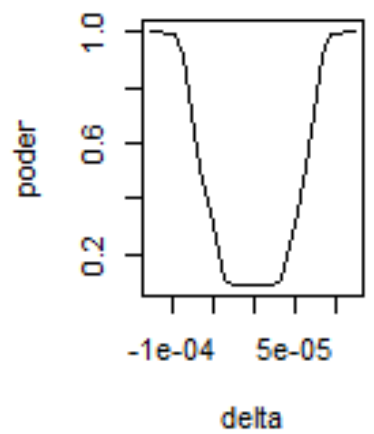

Hip6- F- P. T. Wald

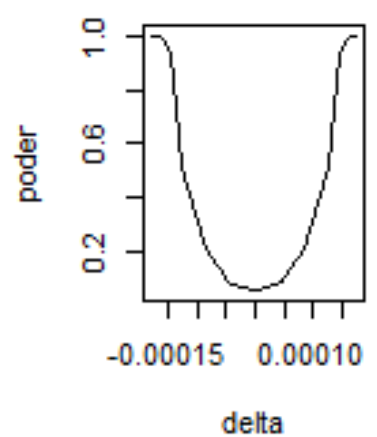

Hip2- F- P. T. RV

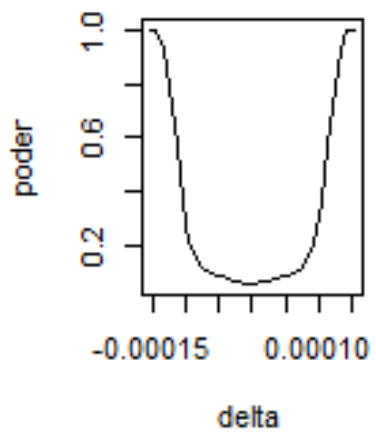

Hip4- F. P. T. RV

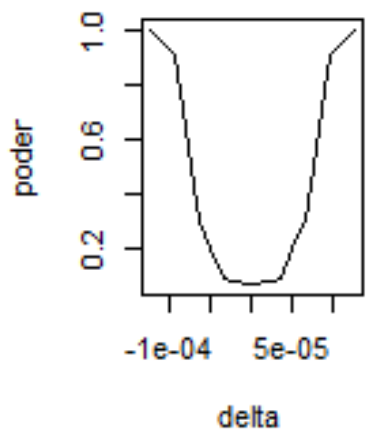

Hip6- F- P. T. RV

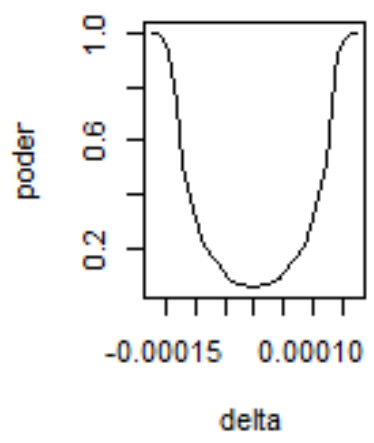

Figura 10: Gráficos do P. T. de Wald e RV para 5 réplicas- 6 laboratórios e 10 rotações $\left(\sigma_{x j s}^{2} \operatorname{com} \sigma_{s}^{2}\right)$. 
Hip1- G- P. T. Wald

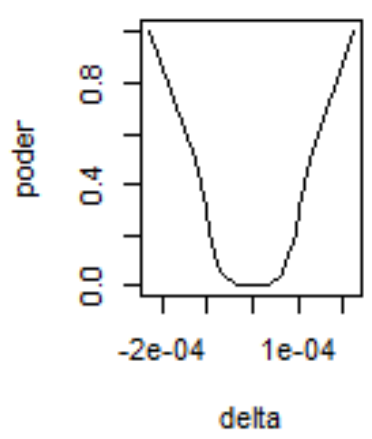

Hip3- G- P. T. Wald

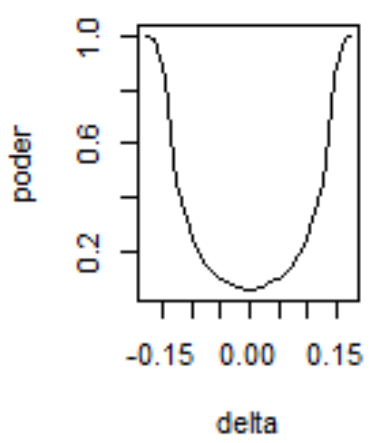

Hip5- G- P. T. Wald

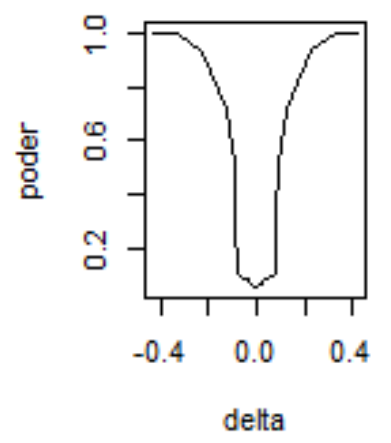

Hip1- G- P. T. RV

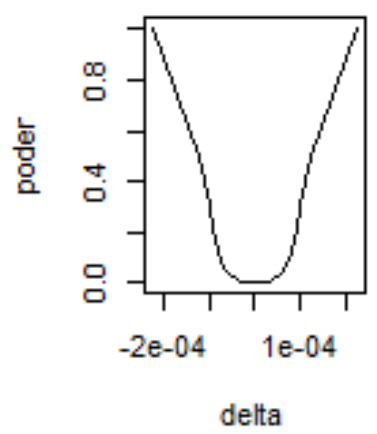

Hip3- G- P. T. RV

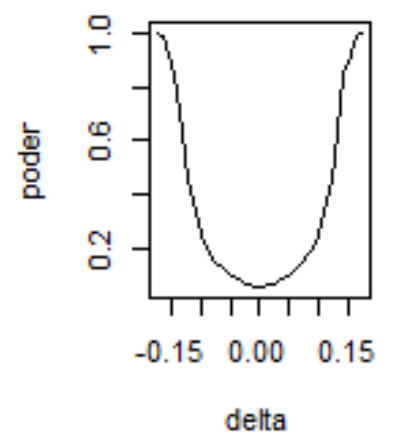

Hip5- G- P. T. RV

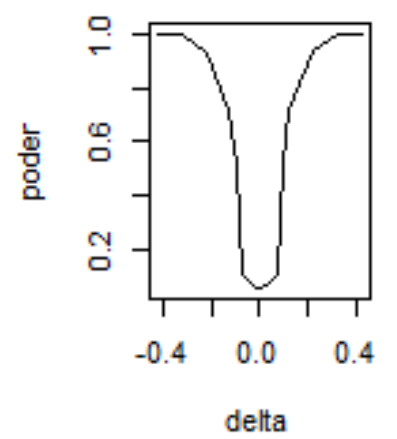

Hip2- G- P. T. Wald

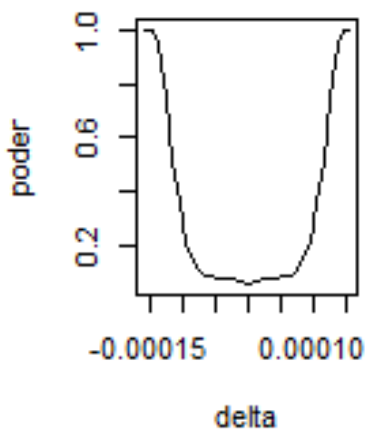

Hip4- G- P. T. Wald

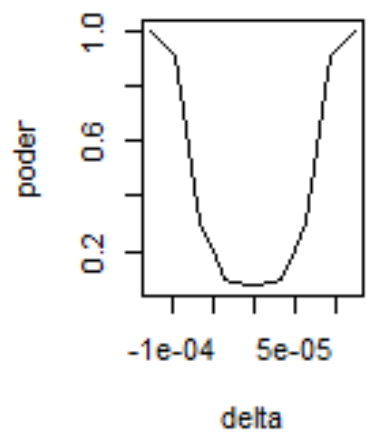

Hip6- G- P. T. Wald

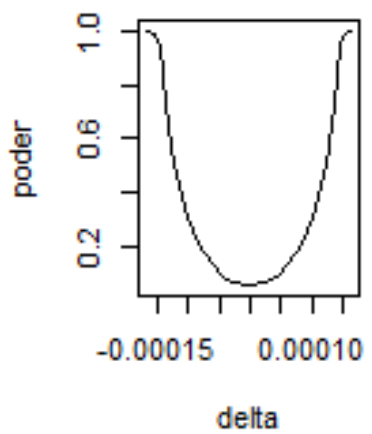

Hip2- G- P. T. RV

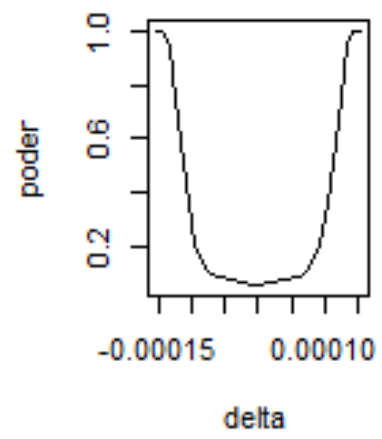

Hip4- G- P. T. RV

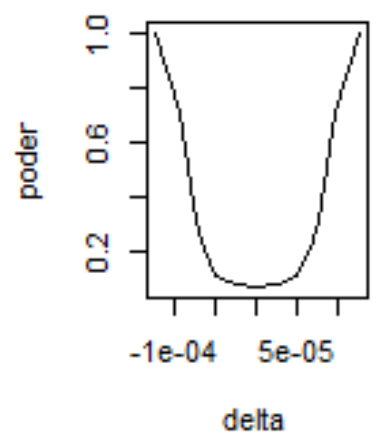

Hip6- G- P. T. RV

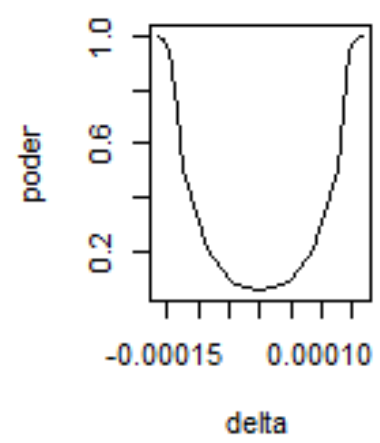

Figura 11: Gráficos do P. T. de Wald e RV para 5 réplicas- 6 laboratórios e 10 rotações $\left(\sigma_{x j s}^{2} \operatorname{com} \sigma^{2}\right)$. 
Hip1- B- P. T. Wald

Hip1- B- P. T. RV

Hip2- B- P. T. Wald

Hip2- B- P. T. RV
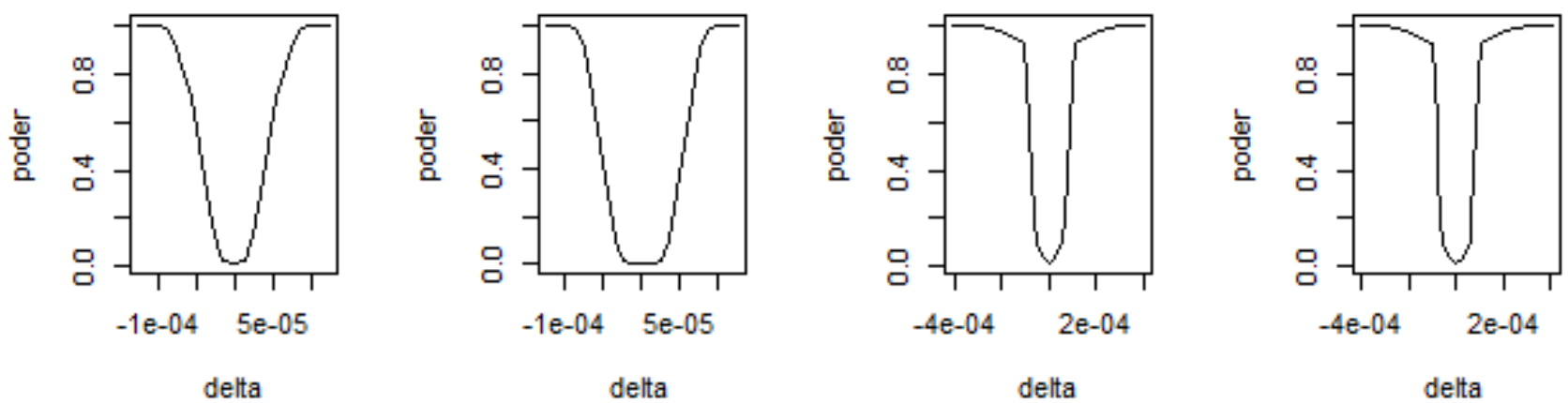

Hip3- B- P. T. Wald

Hip3- B- P. T. RV

Hip4- B- P. T. Wald

Hip4- B- P. T. RV
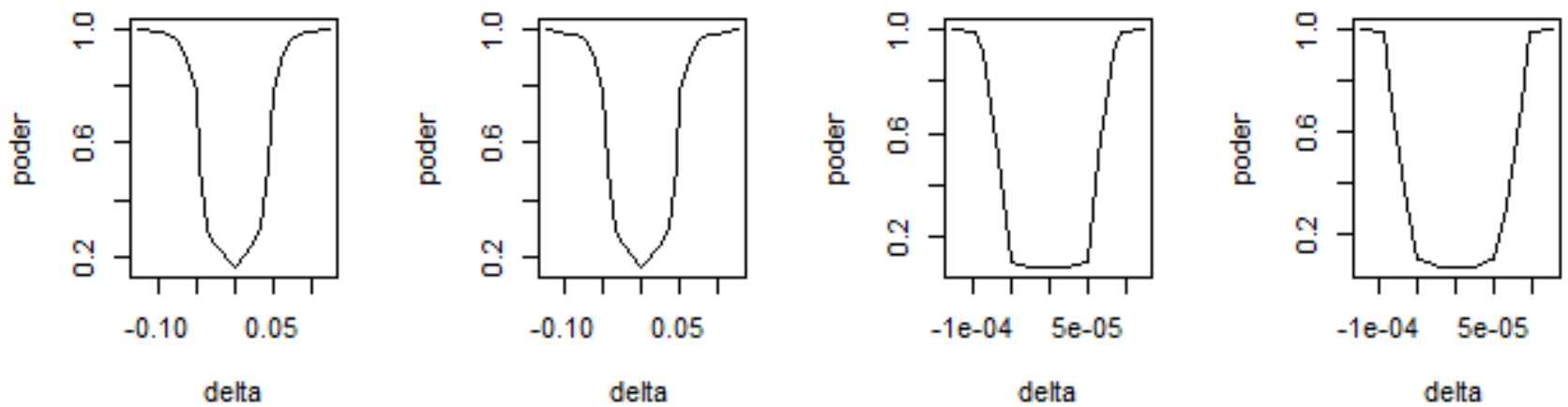

Hip5- B- P. T. Wald

Hip5- B-P. T. RV

Hip6- B- P. T. Wald

Hip6- B- P. T. RV
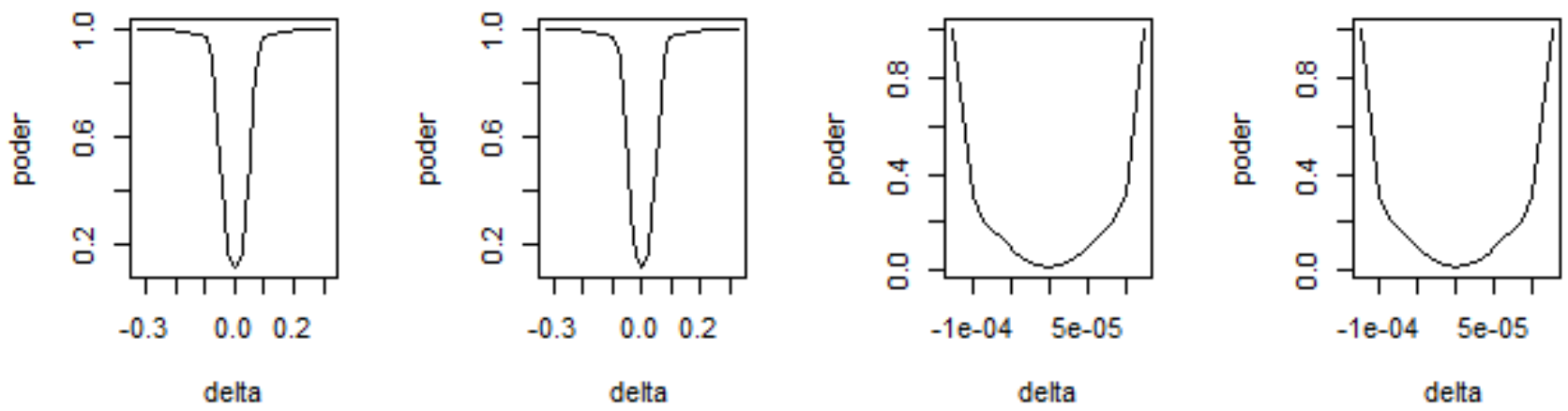

Figura 12: Gráficos do P. T. de Wald e RV para 5 réplicas- 6 laboratórios e 10 rotações $\left(\sigma_{x j s}^{2} \operatorname{com} \sigma_{l}^{2}\right)$. 
Hip1- D- P. T. Wald

Hip1- D- P. T. RV

Hip2- D- P. T. Wald

Hip2- D- P. T. RV
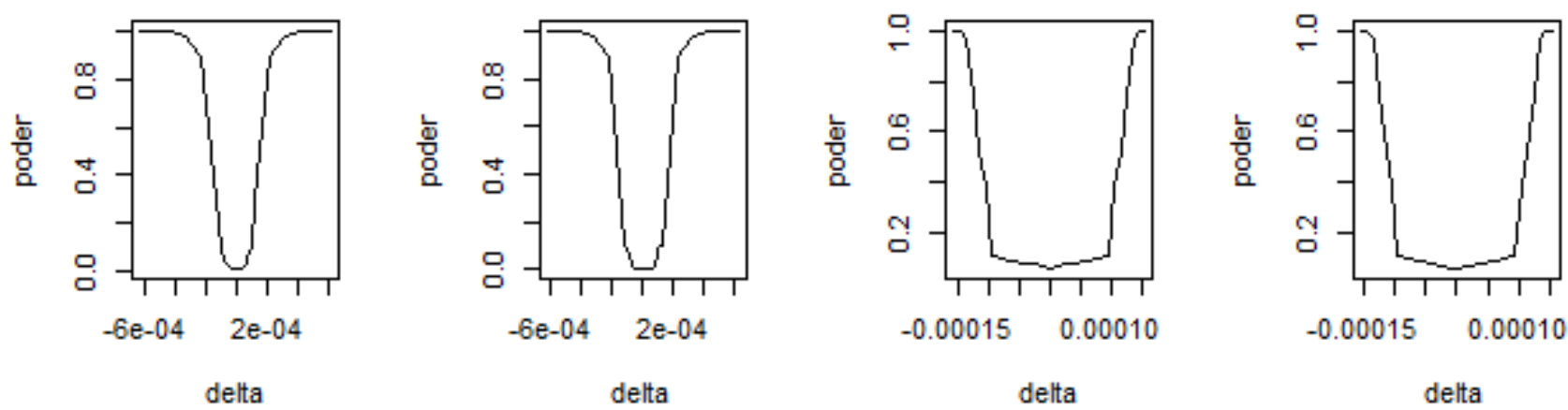

Hip3- D- P. T. Wald

Hip3- D- P. T. RV

Hip4- D- P. T. Wald

Hip4- D- P. T. RV
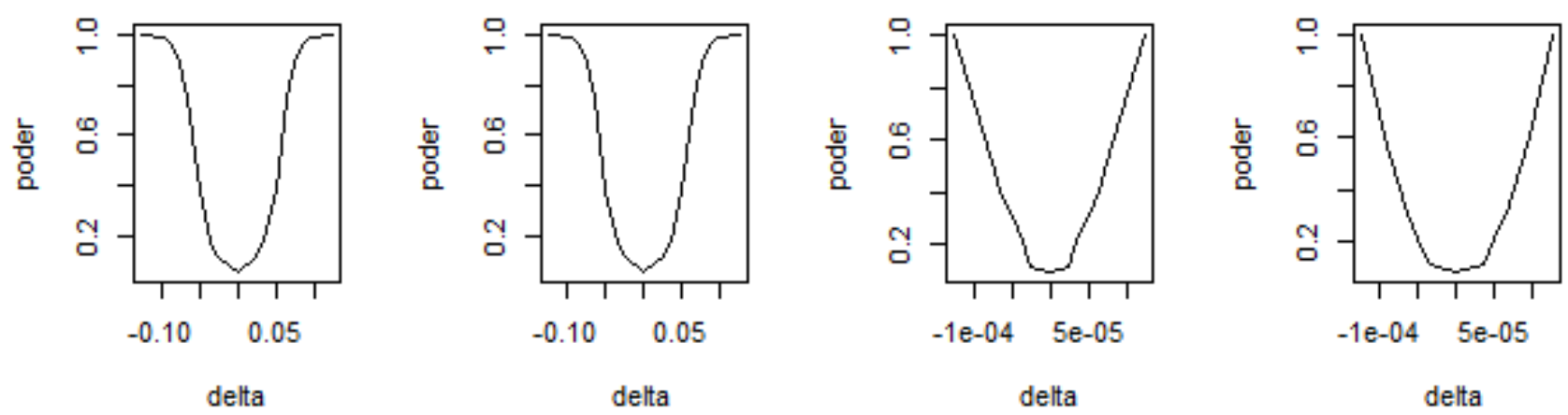

Hip5- D- P. T. Wald

Hip5- D- P. T. RV

Hip6- D- P. T. Wald

Hip6- D- P. T. RV
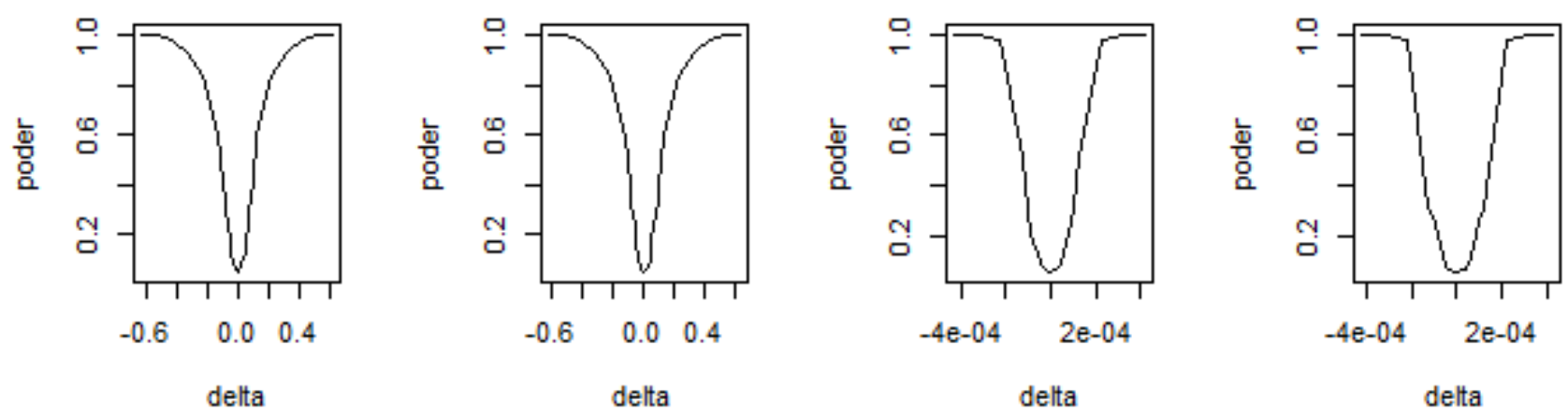

Figura 13: Gráficos do P. T. de Wald e RV para 5 réplicas- 6 laboratórios e 10 rotações $\left(\sigma_{x j}^{2} \operatorname{com} \sigma_{s}^{2}\right)$. 
Hip1- A- P. T. Wald

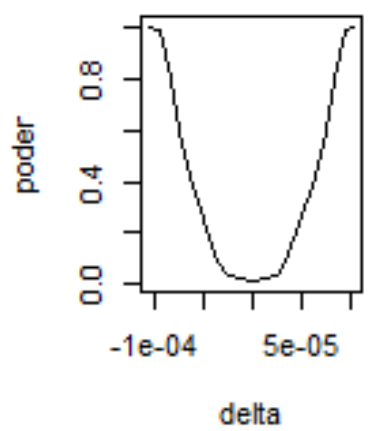

Hip3- A- P. T. Wald

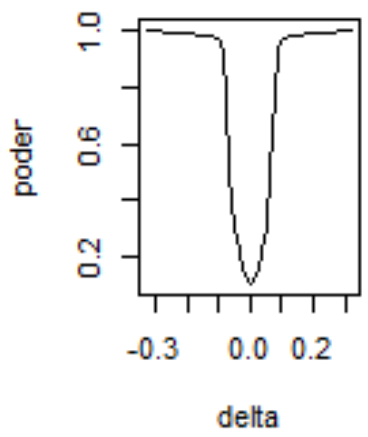

Hip5- A- P. T. Wald

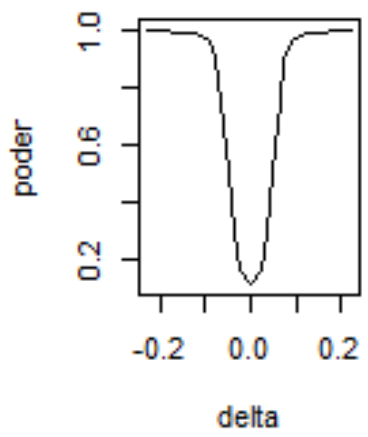

Hip1- A- P. T. RV

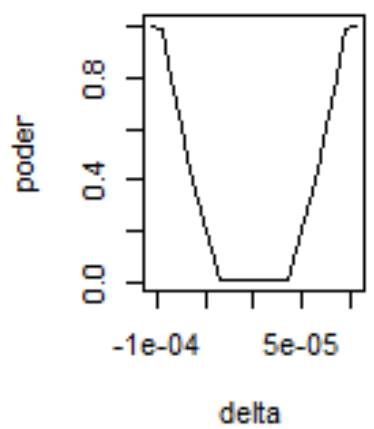

Hip3- A- P. T. RV

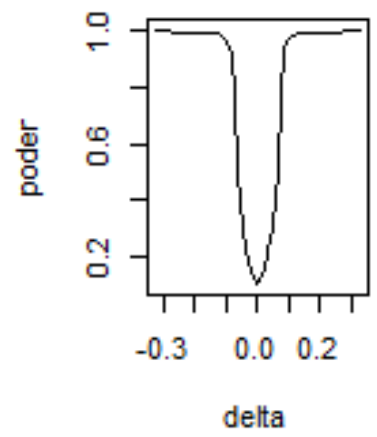

Hip5- A- P. T. RV

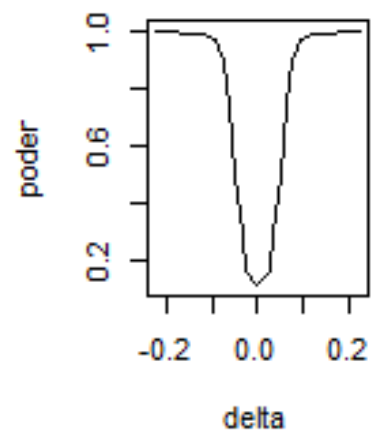

Hip2- A- P. T. Wald

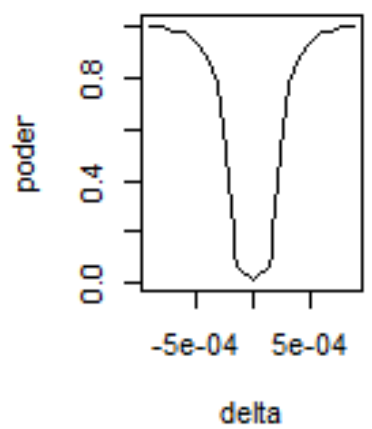

Hip4- A- P. T. Wald

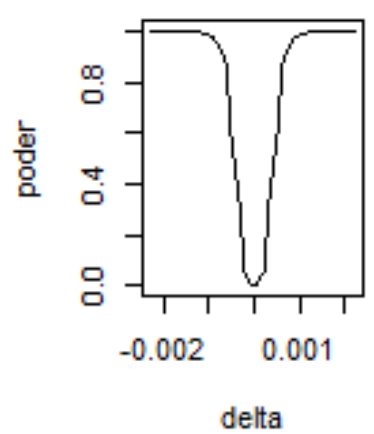

Hip6- A- P. T. Wald

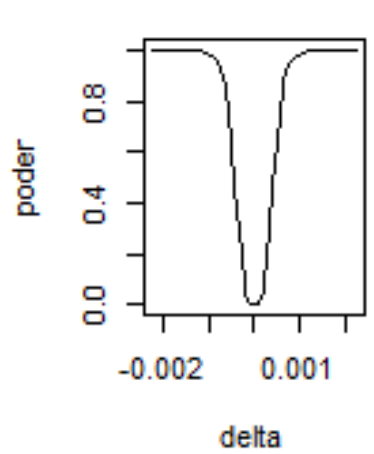

Hip4- A- P. T. RV

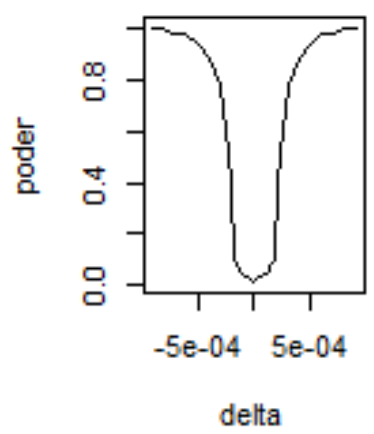

Hip2- A- P. T. RV

Hip6- A- P. T. RV

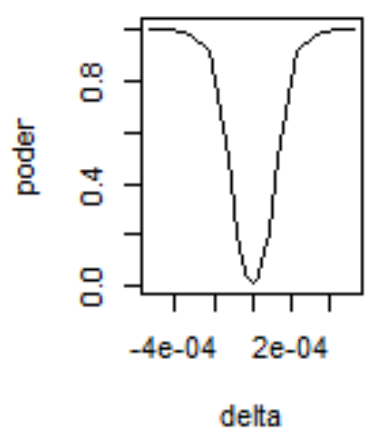

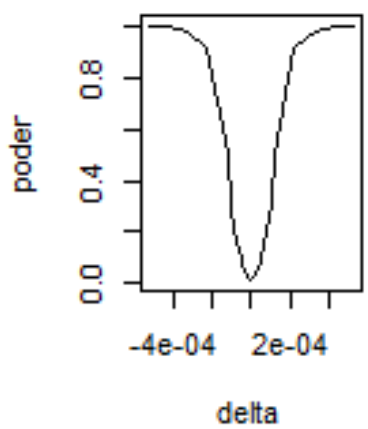

Figura 14: Gráficos do P. T. de Wald e RV para 5 réplicas- 6 laboratórios e 10 rotações $\left(\sigma_{x j}^{2} \operatorname{com} \sigma^{2}\right)$. 
Hip1- E- P. T. Wald

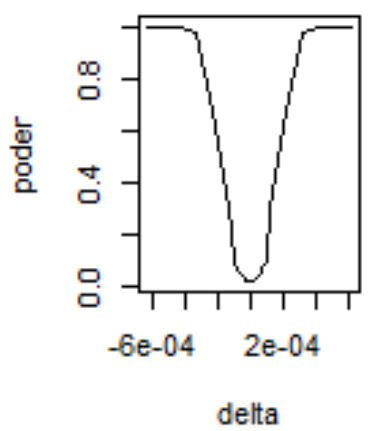

Hip3- E- P. T. Wald

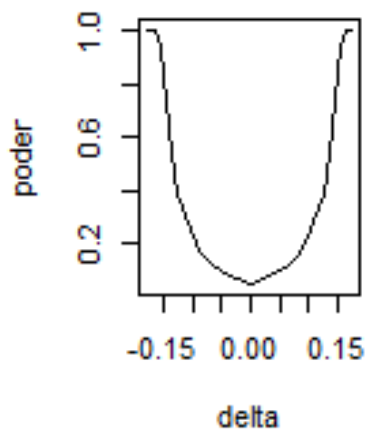

Hip5- E- P. T. Wald

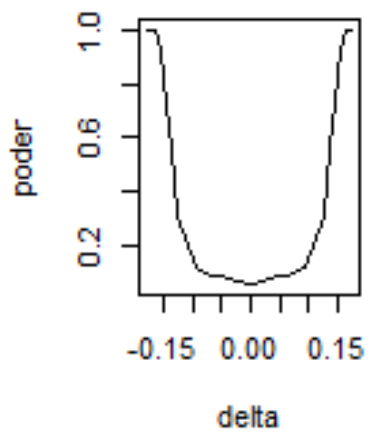

Hip1- E- P. T. RV

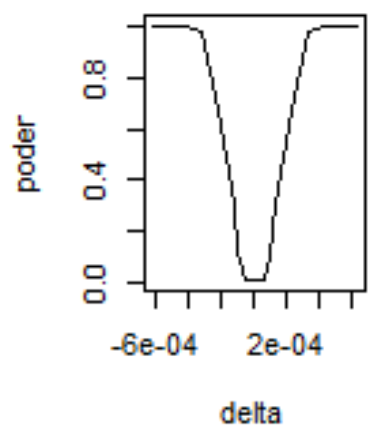

Hip3- E-P. T. RV

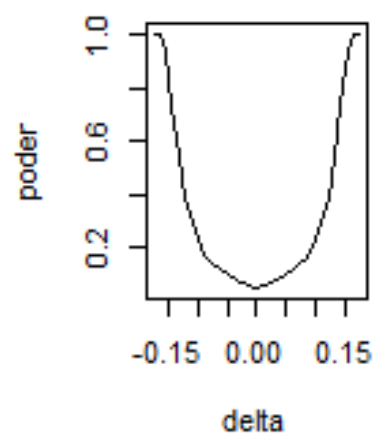

Hip5- E-P. T. RV

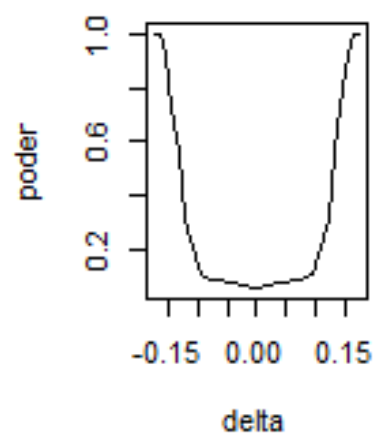

Hip2- E- P. T. Wald

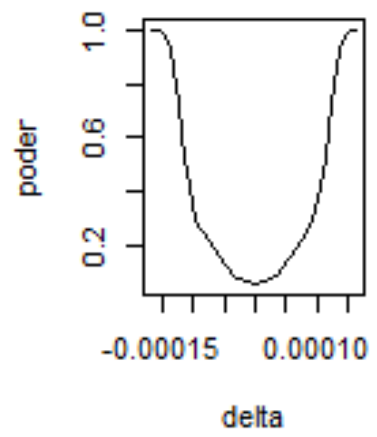

Hip4- E- P. T. Wald

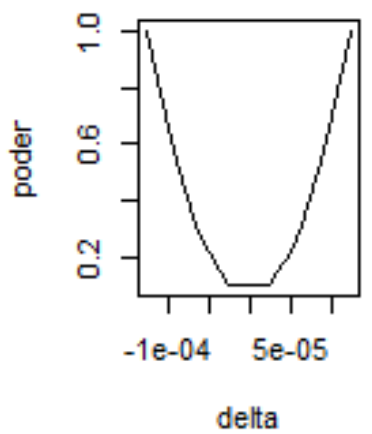

Hip6- E- P. T. Wald

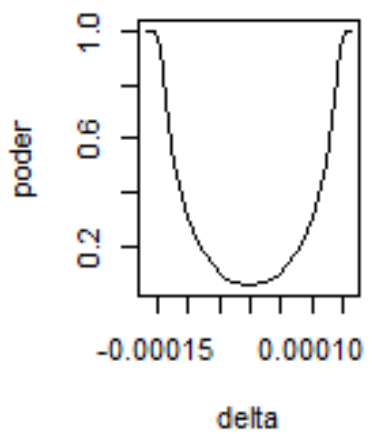

Hip2- E- P. T. RV

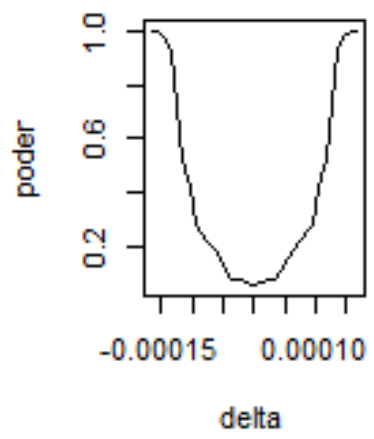

Hip4- E- P. T. RV

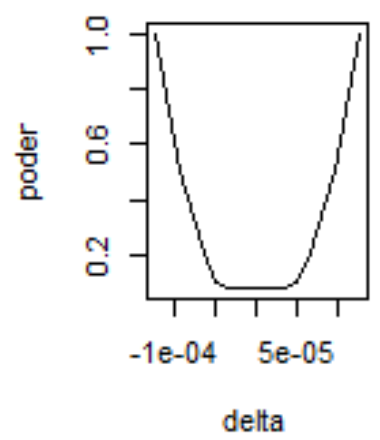

Hip6- E- P. T. RV

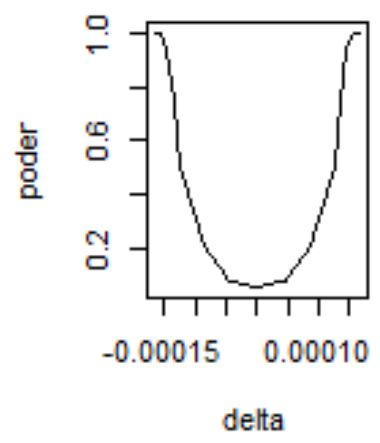

Figura 15: Gráficos do P. T. de Wald e RV para 5 réplicas- 6 laboratórios e 10 rotações $\left(\sigma_{x j}^{2} \operatorname{com} \sigma_{l}^{2}\right)$. 
Hip1- H- P. T. Wald

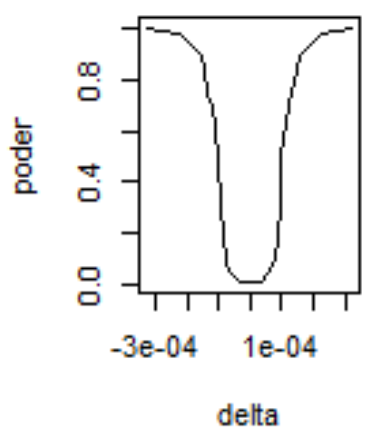

Hip3- H- P. T. Wald

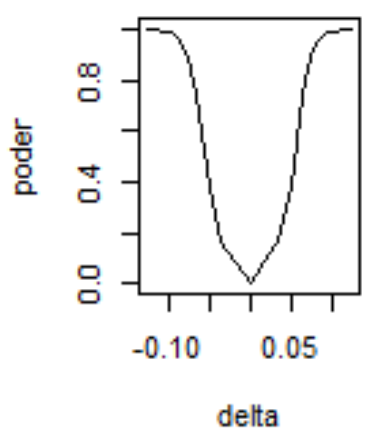

Hip5- H- P. T. Wald

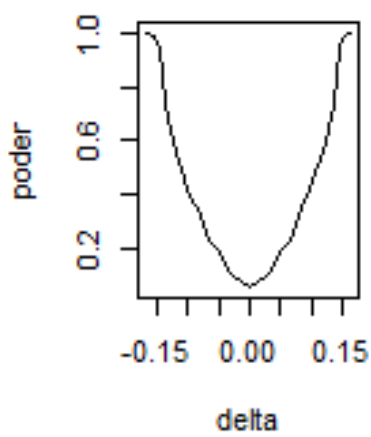

Hip1- H- P. T. RV

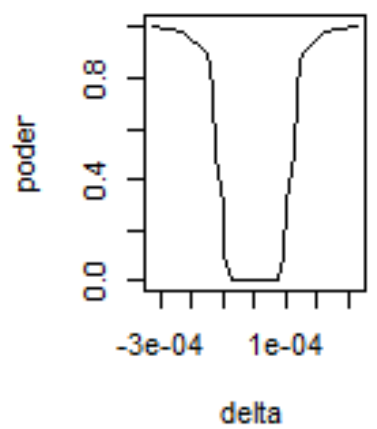

Hip3- H- P. T. RV

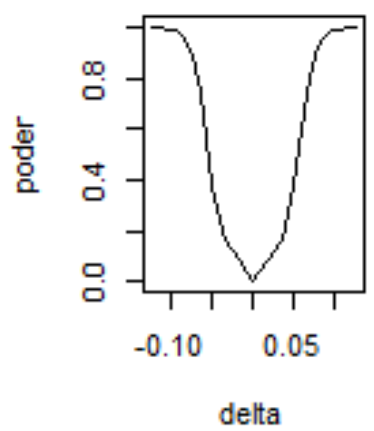

Hip5- H- P. T. RV

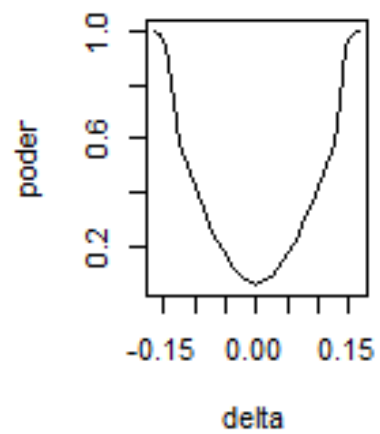

Hip2- H- P. T. Wald

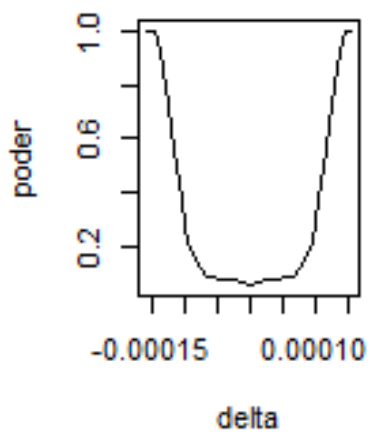

Hip4- H- P. T. Wald

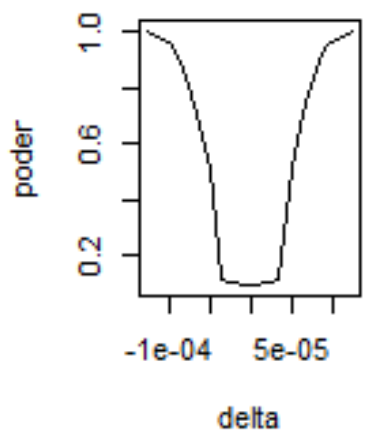

Hip6- H- P. T. Wald

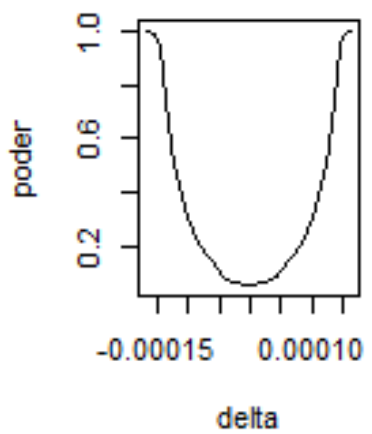

Hip2- H- P. T. RV

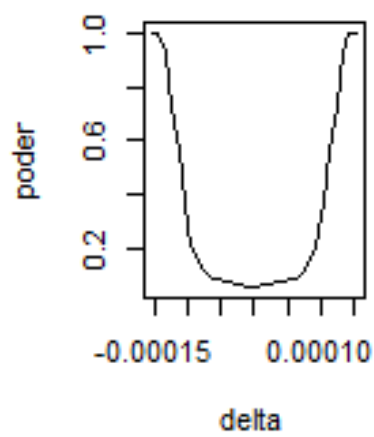

Hip4- H- P. T. RV

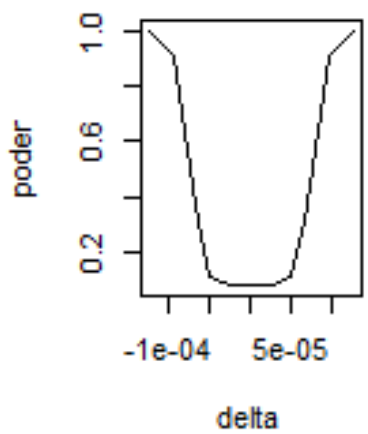

Hip6- H- P. T. RV

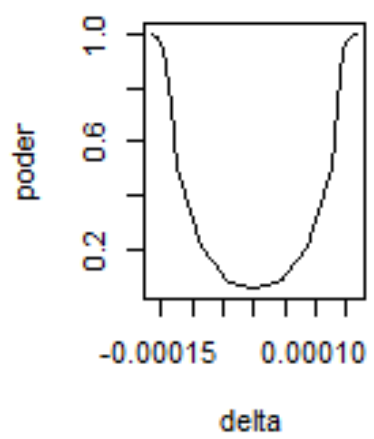

Figura 16: Gráficos do P. T. de Wald e RV para 5 réplicas- 6 laboratórios e 10 rotações $\left(\sigma_{x j l}^{2} \operatorname{com} \sigma_{s}^{2}\right)$. 

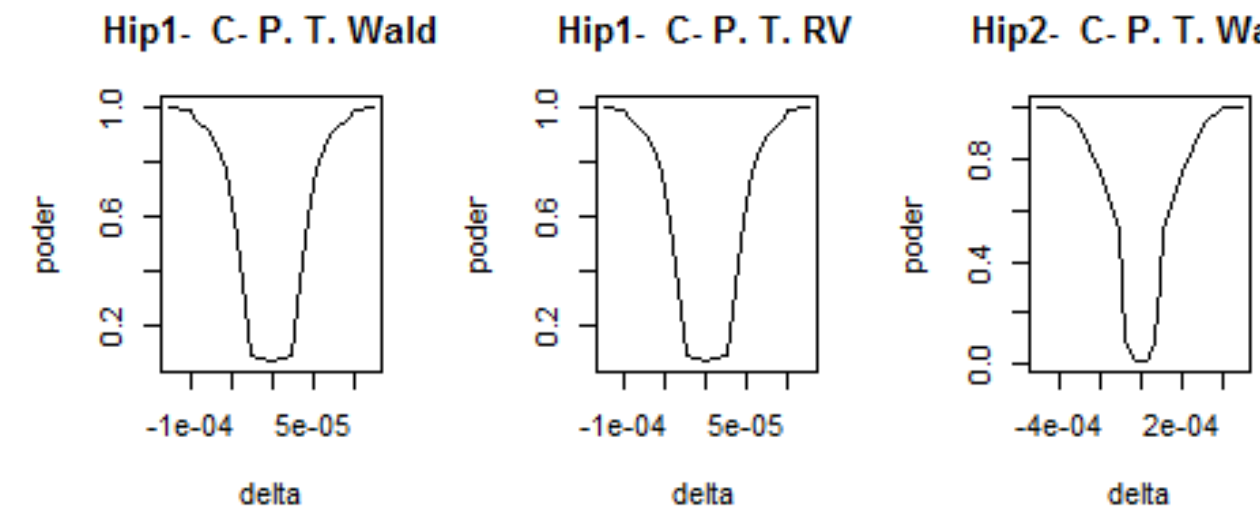

Hip2- C- P. T. RV

Hip3- C- P. T. Wald

Hip3- C. P. T. RV

Hip4- C- P. T. Wald
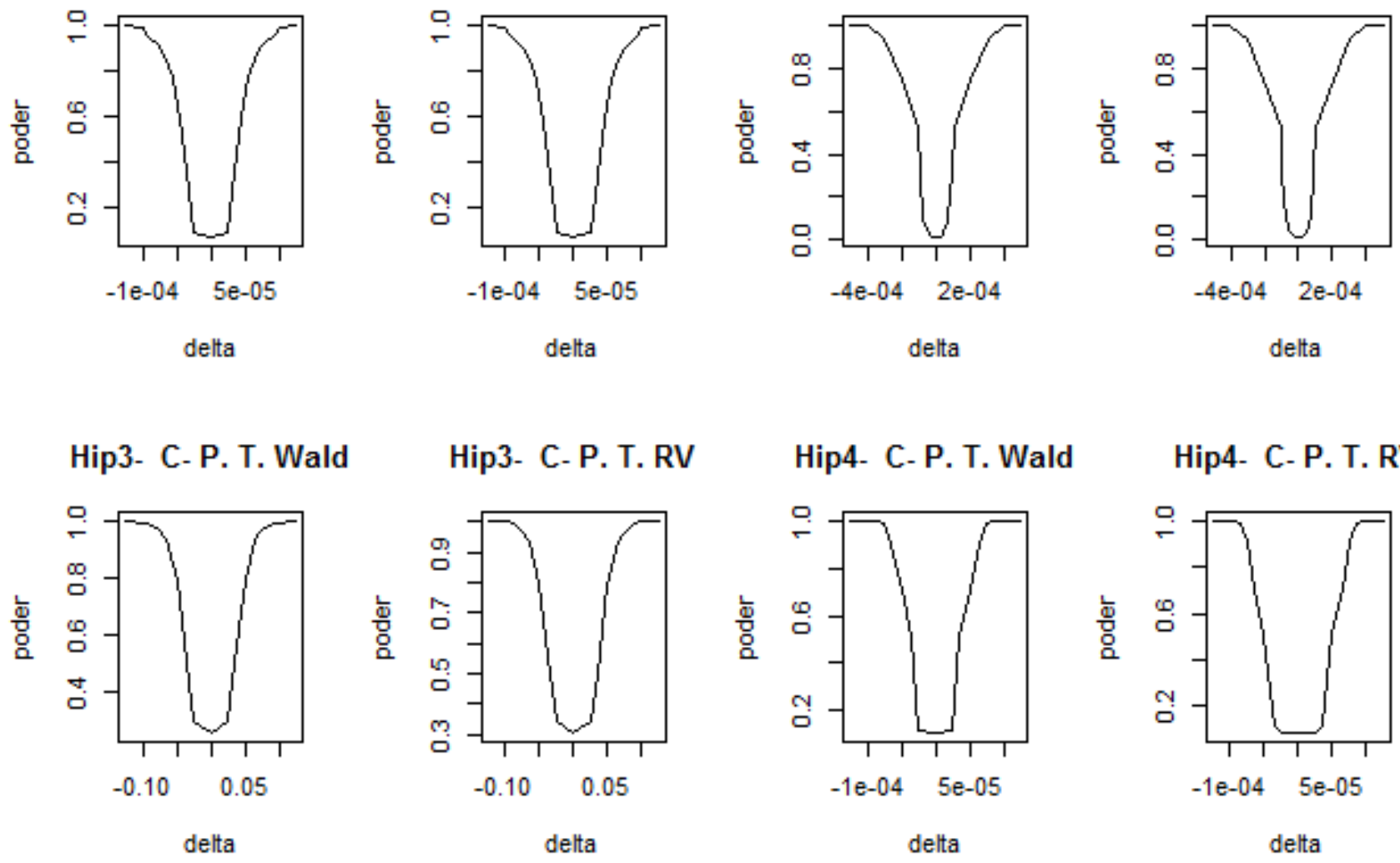

Hip4- C. P. T. RV

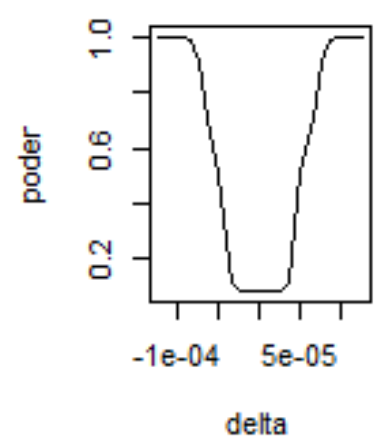

Hip5- C- P. T. Wald

Hip5- C- P. T. RV

Hip6- C- P. T. Wald

Hip6- C- P. T. RV
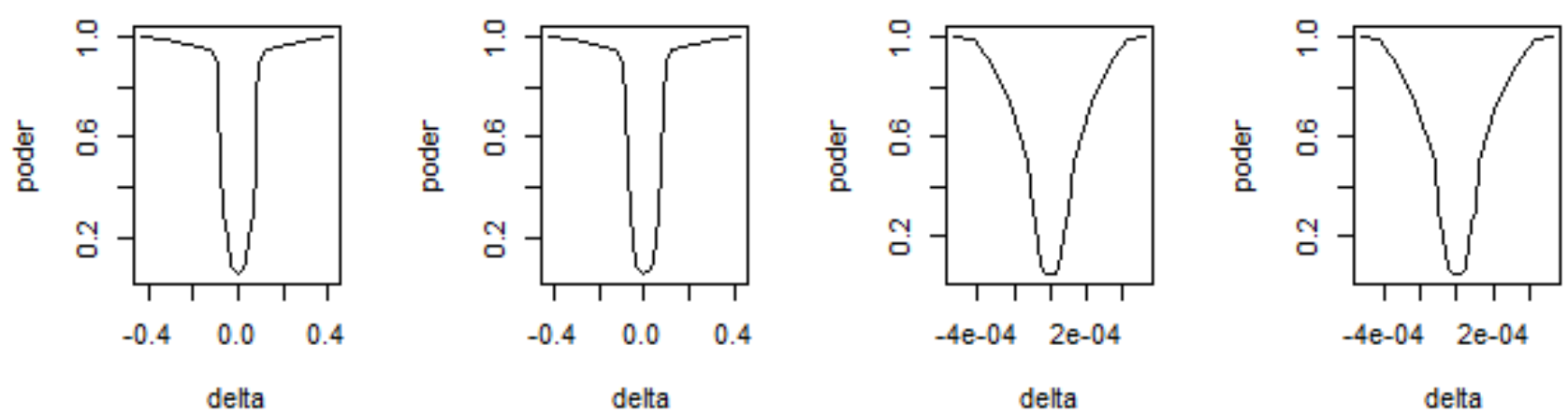

Figura 17: Gráficos do P. T. de Wald e RV para 5 réplicas- 6 laboratórios e 10 rotações $\left(\sigma_{x j l}^{2} \operatorname{com} \sigma^{2}\right)$. 
Hip1- I- P. T. Wald

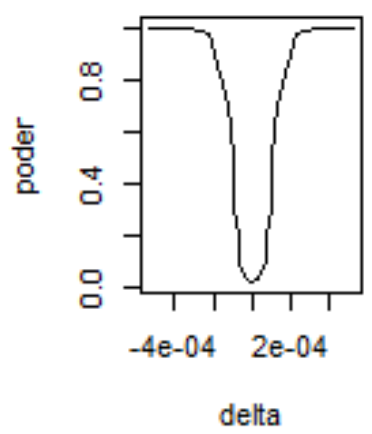

Hip3- I- P. T. Wald

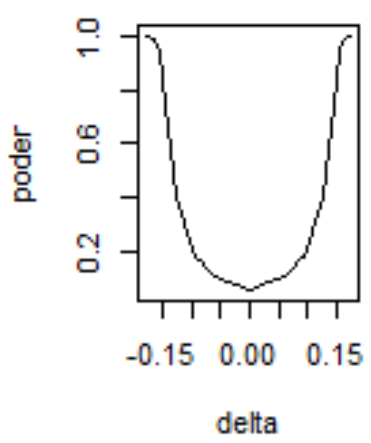

Hip5- I- P. T. Wald

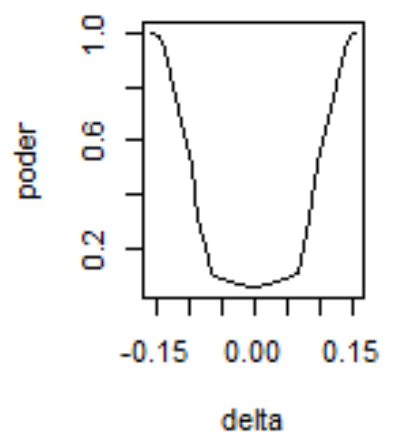

Hip1- I- P. T. RV

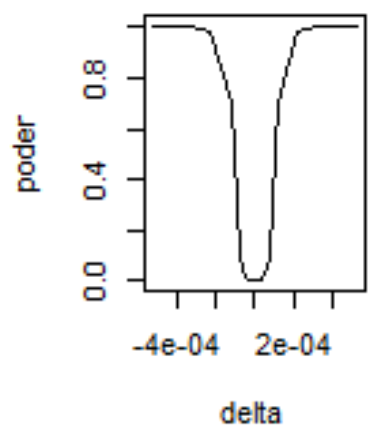

Hip3- I- P. T. RV

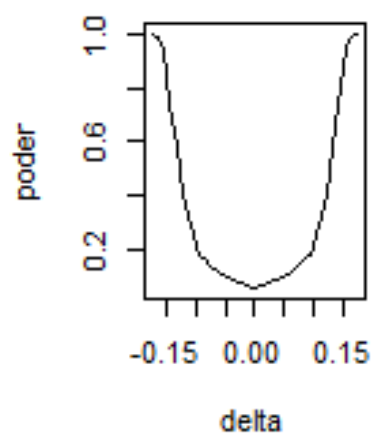

Hip5- I- P. T. RV

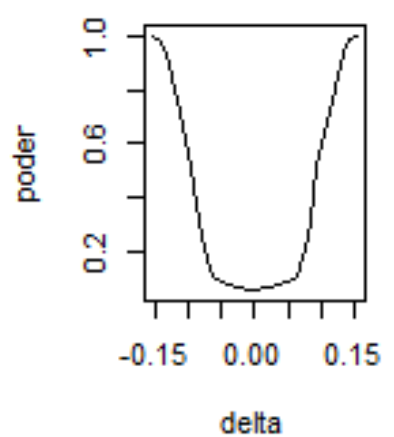

Hip2- I- P. T. Wald

Hip2- I- P. T. RV

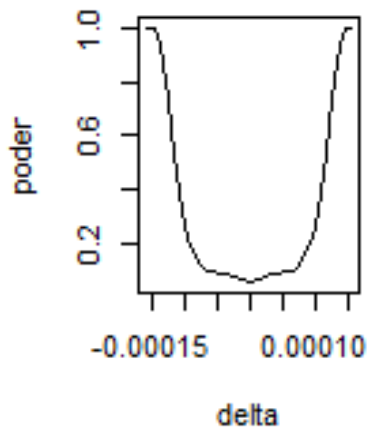

Hip4- I- P. T. Wald

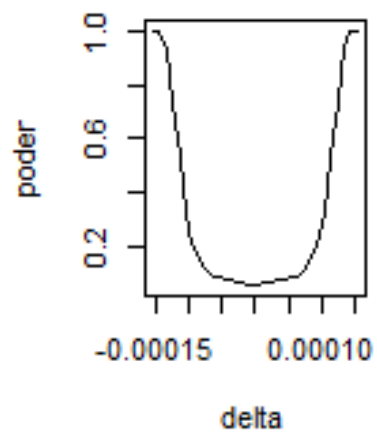

Hip4- I- P. T. RV
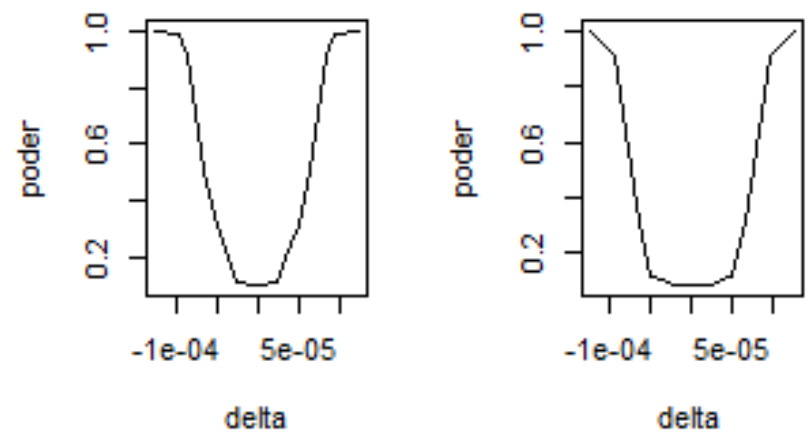

Hip6- I- P. T. RV

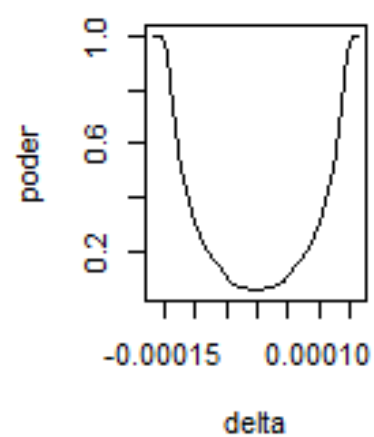

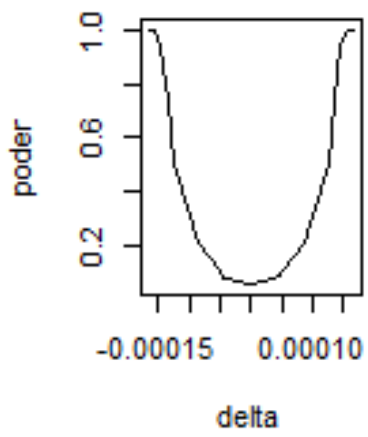

Figura 18: Gráficos do P. T. de Wald e RV para 5 réplicas- 6 laboratórios e 10 rotações $\left(\sigma_{x j l}^{2} \operatorname{com} \sigma_{l}^{2}\right)$.

Comparando os gráficos do poder do teste do modelo onde foi considerada a 
distribuição normal com os gráficos do poder do teste do modelo onde foi considerada a distribuição t de Student, podemos notar que na maioria das vezes o gráfico do poder da t de Student sobe mais rapidamente para 1 e que ao mesmo tempo para valores bem próximos dos valores sob $H_{0}$, o poder da t de Student se mantém e o da normal sobe mais rápido. Isto é de interesse para os dados da nossa aplicação, pelos mesmos motivos descritos em Leão et al. (2009), ou seja, não queremos detectar diferenças que estão "próximas" do valor de referência. 


\section{Conclusão final}

O objetivo deste trabalho foi a comparação dos vícios aditivos e multiplicativos de cada laboratório com uma medida de referência. Ao utilizarmos os dados na aplicação, pudemos concluir que os resultados dos testes usados dependem da distribuição de probabilidade considerada no modelo definido em (1) e (2). Aplicando os valores da aplicação nos estudos de simulação do valor empírico e do poder do teste, sendo que alguns dos valores foram alterados para analisarmos o comportamento dos testes, por exemplo, diferentes valores de $\mathrm{p}$, diferentes valores de $\mathrm{m}$, diferentes valores para $\sigma_{x_{j}}^{2}$ e $\sigma_{i j}^{2}$, concluímos que tais alterações não influenciaram nos resultados. Pudemos notar também que na maioria dos gráficos de poder da t de Student, a curva teve o comportamento esperado (Leão et. al 2009), como descrito na seção 5.2, pois não tínhamos interesse em descredenciar laboratórios por erros muito pequenos. 


\section{Trabalhos Futuros}

Podemos continuar este trabalho, fazendo um estudo de diagnóstico de influência, como por exemplo a influência local que foi proposta por Cook (1986), e consiste em avaliar a robustez das estimativas fornecidas pelo modelo mediante pequenas perturbações aplicadas no modelo ou nos dados. Neste caso, espera-se que o modelo utilizando a distribuição t de Student possa acomodar os possíveis pontos influentes. Uma outra extensão para este modelo seria o uso da classe de distribuições simétricas. 


\section{Referências bibliográficas}

Arellano, R., Bolfarine, H. (1994), 'On score tests in structural regression models', Relatório técnico, RT-MAE 9425, IME-USP.

Barnett, V. D.(1969), 'Simultaneous pairwise linear structural relaionships', Biometrics, 25.

Berkane, M., Kano, Y. \& Bentler, P. M. (1994), 'Pseudo maximum likelihood estimation in elliptical theory- Effects of misspecification', Computational Statistics \& Data Analysis, 18.

Blas, G. B., Bolfarine, H. \& Lachos, V. H.(2011), 'Statistical analysis of controlled calibration model with replicates', Journal of Statistical Computation and Simulation, Vol 1.

Blas, G. B., Sandoval, M. C. and Satomi, O.Y.(2007), 'Homoscedastic controlled calibration model', J Chemometrics, 21.

Bradley, J. J. \& Gart, R. A. (1962), 'The assmptotic properties of ml estimators when sampling from associated populations', Biometrika 49.

Bolfarine, H. \& Rojas, M. G. (1995), 'Structural comparative calibration using the EM algorithm', Journal of Applied Statistics, 22.

Buck, S. F. (1960), 'A method of estimation of missing values in multivariate data suitable for use with an eletronick computer', Journal of the Royal Statistical Society B, 22. 
Cheng, C. L. \& Van Ness, J. W. (1999), Statistical Regression with Measurement Error, 1st edn, Oxford University Press.

Chuong B Do \& Serafim Batzoglou(2008), 'What is the expectation maximization algorithm?', Nature Biotechnology, 26.

Cook, R. D. (1986), 'Assessment of local in?uence (with discussion)', Journal of the Royal Statistical Society, 48.

Dempster, A.P., Laird, N.M., and Rubin, D.B.(1977), 'Maximum likelihood from incomplete dada via the EM algorithm (with discussion)', Journal of the Royal Statistical Society B, 39.

Dolby, G. R. (1976), 'The ultra structural relation: A synthesis of functional and structural relations', Biometrika, 63.

Dunn, G. (1992), 'Design and Analysis of Reliability: the statistical evaluation measurement errors', Edward Arnold. New York.

Fang, K. T., Zhang, Y. T. (1990), 'Generalized Multivariate Analysis', Spinger-Verlag, London.

Fisher, R. A. (1925), 'Statistical Methods for Research Workers', Oliver and Body, Edinburg.

Fuller, A. (1987), Measurement Error Models, 1st edn, Wiley.

Guide 43, ISO/IEC Guide 43 (1997), 'Proficiency testing by interlaboratory comparisons- 
Part I: Development and operation of proficiency testeing schemes.'

Grubbs, F. E. (1948), 'On estimating precision of measuring instruments and product variability', Journal of the American Statistical Association, 48.

Grubbs, F. E. (1973), 'Errors of measurement, precision, accuracy and the statistical comparison of measuring instruments', Technometrics, $\mathbf{1 5 .}$

Hartley, H. O. (1958), 'Maximum Likelihooh estimatoin form incomplete data', Biometrics, 14.

ISO/IEC Guide 43-1 (1997). 'Proficiency testing by interlaboratory comparisons Part I: Development and operation of proficiency testing schemes'.

Jaech, J. L. (1985), 'Statistical analysis of measurement errors', Exxon Monographs, John Wiley, New York.

Johnson, R. A \& Wichern,D. W. (1998), 'Applied Multivariate Statistical Analysis'., 4th edn, Prentice Hall, New Jersey.

Kelly, G. (1984), 'The influence function in the errors in varaiable problem', Then Annals of Statistics, 12.

Kelly, G. (1985), 'Use of structural equation model in assessing the reliability of a new measurement technique', Applied Statistics, 34.

Kendall, M.G. \& Stuart, A. (1961), 'The advanced theory of statistics', London: Griffin. 
Kendall, M.G. \& Stuart, A. (1973), 'The advanced theory of statistics', Vol 1, 2, 4th edn, London: Griffin.

Labra, V.F. \& Aoki, R. \& Boldarine, H. (2005), 'Local Influence in Null Intercept Measurement Error Regression Under a Student-T Model', Journal of Applied Statistics, Vol 32, 7.

Leão, D. P. J., AOKI, R., SILVA, G. F. (2009), 'Statistical Analysis of Proficiency testing results under elliptical distribuitions.', Computational Statistics \& Data Analysis, 53.

Lesaffre, E. , Verbeke, G. (1998), 'Local Influence in Linear Mixed Models.', Biometrics, 54.

Leurgans, S. (1980), 'Evaluating laboratory measurement techniques in Biostatistics.', Case book.

Lima, C.R.O. (1996), 'Calibração Absoluta com erros nas variáveis', Dissertação de Mestrado-IME.

Little, R. J. (1988), 'Robust estimation of the mean and covariance matrix from data missing values', Applied Statistics, 37.

McKendrick, A.G.(1926), 'Applications of Mathematics to medical problems', Proceedings of the Endinburg Mathematical Society, 44.

McLachlan, G.F. \& Krishnan, T.(1997), 'The EM algorithm and Extensions'. 
Montanari, A. O. (2006), 'Análise dos resultados de ensaios de proficiência via modelos de regressão com variável explicativa aleatória.', Dissertação de Mestrado-ICMC.

Moran, P. A. P (1971), 'Estimating structural and functional relationships', Journal of Multivariate Analysis, 1.

Neves, L.; Martins, A.; Antunes, C.; Dias, L. (2004), 'Using SSM to rethink the analysis of energy efficiency initiatives.', Journal of the Operational Research Society, Vol 55, 9.

Newcomb, S. (1886), 'A gerenalized theory of the combination of observation so as to obtain the best result', American Journal of Mathematics, 8.

Oliveira, P.T.M.S. (2001), 'Estimação e testes de hipóteses em calibração comparativa', Dissertação de Mestrado-IME-USP.

Rao, C. R. (1973), 'Linear statistical inference and its applications'. New York: John Wiley.

Richardson, J.T.E. (1996) Handbook of Qualitative Research Methods for Psychology and the Social Sciences. BPS Books.

Rojas, M.J.G. (1995), 'Calibração Comparativa Estrutural e Funcional.', Dissertação de Doutorado-IME-USP.

Rubin, B.D (1983), 'Iteratively reweighted least squares', Encyclopedia og Statistical Sciences, 4 . 
Russo, C. M. (2006), 'Análise de um modelo de regressão com erros nas variáveis multivariado com intercepto nulo', Dissertação de Mestrado-ICMC-USP.

Searle, S.R.(2006), Linear Models, Wiley Classics Library.

Shukla, G. K. (1972), 'On the problem of calibration', Technometrics, Vol 14, 3.

Sobral, T. E. L., Barreto, G. (2011), 'Análise dos critérios de informação para seleção de ordem em modelos auto-regressivos.', DINCON.

Sutradhar, B. C., Ali, M. M. (1986). 'Estimation of the parameter of regression model with a multivariate $t$ error variable', Communications in Statistics- Theory and Methods, 15.

Yang, C. (2008), 'A study of the calibration-inverse prediction problem in a mixed model setting ', Dissertação de mestrado- Kansas State University

Zavala, A. A. (2001), 'Análise comparativa dos algoritmos EM e SIMEX nos modelos lineares mistos aplicados ao análise de regressão com erros nas variáveis.', Dissertação de mestrado-IME-USP.

Zhu, H. and Lee, S. (2003), 'Local influence for incomplete-data models.', Journal of the Royal Statistical Society, B, 63. 


\section{Apêndice A}

Teorema (4.1). Seja $(\Omega, F, P)$ um espaço de probabilidade e $z_{i}$ um vetor aleatório com função de densidade de probabilidade $f\left(\boldsymbol{z}_{i}, \boldsymbol{\theta}\right)$, onde $\boldsymbol{z}_{i}$ assume valores em uma região $R_{i}$ independente de $\boldsymbol{\theta}=\left(\theta_{1}, \ldots, \theta_{k}\right) \in \Theta$ para todo $i=1, \ldots, p$ e $\boldsymbol{z}_{i}$ independete de $\boldsymbol{z}_{j}$, para $i \neq j$. Não é necessário que cada $f_{i}$ dependa de todos os parâmetros $\theta_{1}, \ldots, \theta_{k}$. Sejam $\boldsymbol{z}_{i \mathbf{1}}, \ldots, \boldsymbol{z}_{i \boldsymbol{i}} n_{i}$ vetore de observações independetes de $\boldsymbol{z}_{i}, i=1, \ldots, p$, com função de densidade de probabilidade $f_{i_{j}}\left(\boldsymbol{z}_{i_{j}}, \boldsymbol{\theta}\right)$ para $i=1, \ldots, p$ e $j=1, \ldots, n_{i}$. A função densidade de probabilidade conjunta é dada por

$$
f(\boldsymbol{z}, \boldsymbol{\theta})=\prod_{i=1}^{p} \prod_{j=1}^{n_{i}} f_{i}\left(\boldsymbol{z}_{i_{j}}, \boldsymbol{\theta}\right)
$$

Supondo válidas as condições de reguralidade:

1. Para quase todo $\boldsymbol{z}_{i} \in R_{i}$ e para todo $\boldsymbol{\theta} \in \Theta$,

$$
\frac{\partial \log f_{i}}{\partial \theta_{r}}, \frac{\partial^{2} \log f_{i}}{\partial \theta_{r} \partial \theta_{s}} e \frac{\partial^{3} \log f_{i}}{\partial \theta_{r} \partial \theta_{s} \partial \theta_{t}}
$$

existem para $r, s, t=1, \ldots, k ; i=1, \ldots, p$

2. Para toda densidade $f_{i}$, para quase todo $\boldsymbol{z}_{i} \in R_{i}$ e para todo $\boldsymbol{\theta} \in \Theta$

$$
\left|\frac{\partial f_{i}}{\partial \theta_{r}}\right|<F_{i r}\left(\boldsymbol{z}_{i}\right),\left|\frac{\partial^{2} \log f_{i}}{\partial \theta_{r} \partial \theta_{s}}\right|<F_{\text {irs }}\left(\boldsymbol{z}_{i}\right) \text { e }\left|\frac{\partial^{3} \log f_{i}}{\partial \theta_{r} \partial \theta_{s} \partial \theta_{t}}\right|<H_{\text {irst }}\left(\boldsymbol{z}_{i}\right),
$$

onde $F_{\text {ir }}\left(z_{i}\right), F_{\text {irs }}\left(z_{i}\right)$ são integráveis em $R_{i}$ e

$$
\int_{R_{i}} H_{i r s t}\left(z_{i}\right) f_{i} d z_{i}<M_{i}
$$

$(i=1, \ldots, p ; r, s, t=1, \ldots, k)$, onde $M_{i}$ são constantes positivas e finitas. Analogamente, para toda função de probabilidade $f_{i}$,

$$
\sum_{z_{i} \in R_{i}} \frac{\partial f_{i}}{\partial \theta_{r}} e \sum_{z_{i} \in R_{i}} \frac{\partial^{2} f_{i}}{\partial \theta_{r} \theta_{s}}
$$


convergem uniformemente para todo $\boldsymbol{\theta} \in \Theta e$

$$
\left|\frac{\partial^{3} f_{i}}{\partial \theta_{r} \theta_{s} \theta_{t}}\right|<H_{\text {irst }}\left(\boldsymbol{z}_{i}\right)
$$

onde

$$
\sum_{z_{i} \in R_{i}} H_{i r s t}\left(z_{i}\right) f_{i}<M_{i}
$$

para todo $\boldsymbol{\theta} \in \Theta(r, s, t=1 \ldots, k ; i=1, \ldots, p)$ e com $M_{i}$ constantes positivas e finitas. Estas condições permitirão intercambiar a ordem da diferenciação e integração ou somatória.

3. Para todo $\boldsymbol{\theta} \in \Theta$, a matriz $J=\left[J_{r s}(\boldsymbol{\theta})\right]$ com

$$
J_{r s}(\boldsymbol{\theta})=\sum_{i=1}^{p} m_{i} \int_{R_{i}} \frac{\partial \log f_{i}}{\partial \theta_{r}} \frac{\partial \log f_{i}}{\partial \theta_{s}} f_{i} d \boldsymbol{z}_{i}
$$

é positiva definida com determinante finito (para o caso discreto basta substituir a integração por somatória), com $m_{i}=\frac{n_{i}}{N}$ e $N=\sum_{i=1}^{p} n_{i}$.

Então, se $\hat{\boldsymbol{\theta}}$ é o estimador de máxima verossimilhança de $\boldsymbol{\theta}_{0}$ (verdadeiro valor de $\boldsymbol{\theta})$, temos que $\sqrt{N}\left(\hat{\boldsymbol{\theta}}-\boldsymbol{\theta}_{0}\right)$ tem distribuição assintótica (quando $\left.N->\infty\right)$ normal multivariada com vetor de médias 0 e matriz de covariâncias $J_{0}^{-1}$, onde

$$
J_{0}=\left.\sum_{i=1}^{p} m_{i} E\left(\frac{\partial \log f_{i}}{\partial \theta_{r}} \frac{\partial \log f_{i}}{\partial \theta_{s}}\right)\right|_{\boldsymbol{\theta}=\boldsymbol{\theta}_{0}}=-\left.\sum_{i=1}^{p} m_{i} E\left(\frac{\partial^{2} \log f_{i}}{\partial \theta_{r} \partial \theta_{s}}\right)\right|_{\boldsymbol{\theta}=\boldsymbol{\theta}_{0}}
$$

Prova em Bradley \& Gart (1962).

Teorema (5.1). Seja $(\Omega, F, P)$ um espaço de probabilidade e $z_{i}$ um vetor aleatório com função de densidade de probabilidade $f\left(\boldsymbol{z}_{i}, \boldsymbol{\theta}\right)$, onde $\boldsymbol{z}_{i}$ assume valores em uma região $R_{i}$ independente de $\boldsymbol{\theta}=\left(\theta_{1}, \ldots, \theta_{k}\right) \in \Theta$ para todo $i=1, \ldots, p$ e $\boldsymbol{z}_{i}$ independete de $\boldsymbol{z}_{j}$, para $i \neq j$, como no Teorema 4.1. Novamente, não é necessário que cada $f_{i}$ dependa de todos os parâmetros $\theta_{1}, \ldots, \theta_{k}$. Sejam $\boldsymbol{z}_{i \mathbf{1}}, \ldots, \boldsymbol{z}_{i \boldsymbol{i}} n_{i}$ vetore de observações independetes de $\boldsymbol{z}_{i}, i=1, \ldots, p$, com função de densidade de probabilidade $f_{i_{j}}\left(\boldsymbol{z}_{i_{j}}, \boldsymbol{\theta}\right)$ para $i=1, \ldots, p$ e $j=1, \ldots, n_{i}$. A função densidade de probabilidade conjunta é dada por 


$$
f(\boldsymbol{z}, \boldsymbol{\theta})=\prod_{i=1}^{p} \prod_{j=1}^{n_{i}} f_{i}\left(\boldsymbol{z}_{i_{j}}, \boldsymbol{\theta}\right)
$$

Supondo válidas as condições de reguralidade descritas no Teorema 4.1, temos que: $(I) \sqrt{N}\left(\hat{\boldsymbol{\theta}}-\boldsymbol{\theta}_{0}\right) \stackrel{N \rightarrow \infty}{\rightarrow} \mathcal{N}_{k}\left(\boldsymbol{0}, J^{-1}\left(\boldsymbol{\theta}_{0}\right)\right)$, onde $J\left(\boldsymbol{\theta}_{0}\right)$ é dado por

$J_{\boldsymbol{\theta}_{0 r s}}=\left.\sum_{i=1}^{p} m_{i} E\left(\frac{\partial \log f_{i}}{\partial \theta_{r}} \frac{\partial \log f_{i}}{\partial \theta_{s}}\right)\right|_{\boldsymbol{\theta}_{=} \boldsymbol{\theta}_{0}}=-\left.\sum_{i=1}^{p} \frac{n_{i}}{N} E\left(\frac{\partial^{2} \log f_{i}}{\partial \theta_{r} \partial \theta_{s}}\right)\right|_{\boldsymbol{\theta}_{=} \boldsymbol{\theta}_{0}}$,

com $\hat{\boldsymbol{\theta}}$ o estimador de máxima verossimilhança de $\boldsymbol{\theta}$ e $\boldsymbol{\theta}_{0}$ o verdadeiro valor de $\boldsymbol{\theta}$.

Seja

$$
H_{0}: \boldsymbol{h}(\boldsymbol{\theta})=\mathbf{0} \text { versus } H_{1}: \boldsymbol{h}(\boldsymbol{\theta}) \neq \mathbf{0}
$$

onde $\boldsymbol{h}(\boldsymbol{\theta})=\left(\theta_{1}, \ldots, \theta_{r}\right)^{\prime}: \mathbb{R}^{k} \rightarrow \mathbb{R}^{r}(r \leq k)$ é tal que

(i) Existem $k-r$ funções adicionais $h^{r+1}(\boldsymbol{\theta}), \ldots, h^{r}(\boldsymbol{\theta})$ tal que as relações inversas $\theta_{1}\left(\boldsymbol{h}^{*}\right), \ldots, \theta_{k}\left(\boldsymbol{h}^{*}\right)$ existem, com $\boldsymbol{h}^{*}=\left(h_{1}(\boldsymbol{\theta}), \ldots, h_{k}(\boldsymbol{\theta})\right)$.

(ii) as derivadas parciais de primeira e segunda ordem de $h_{1}(\boldsymbol{\theta}), \ldots, h_{k}(\boldsymbol{\theta})$ são funções uniformemente continuas e limitadas de $\boldsymbol{\theta}$.

(iii) o maior limite inferior do valor absoluto do jacobiano $\frac{\partial\left(h_{1}, \ldots, h_{k}\right)^{\prime}}{\partial\left(\theta_{1}, \ldots, \theta_{k}\right)}$ é positivo.

Seja $\boldsymbol{H}(\boldsymbol{\theta})=\frac{\partial \boldsymbol{h}(\boldsymbol{\theta})^{\prime}}{\partial \boldsymbol{\theta}}$ uma matriz $(k \times r)$ continua em $\boldsymbol{\theta}$ com posto $r, \hat{\boldsymbol{\theta}}$ o estimador de máxima verossimilhança de $\boldsymbol{\theta}$ e $\overline{\boldsymbol{\theta}}$ o estimador de máxima verossimilhança restrito a $H_{0}$, ou seja, tal que $h(\overline{\boldsymbol{\theta}})=0$, e seja

Estatística de Wald

$$
\text { - } Q_{W}=n \boldsymbol{h}(\hat{\boldsymbol{\theta}})^{t}\left[\boldsymbol{H}(\hat{\boldsymbol{\theta}})^{t}[I(\hat{\boldsymbol{\theta}})]^{-1} \boldsymbol{H}(\hat{\boldsymbol{\theta}})\right]^{-1} \boldsymbol{h}(\hat{\boldsymbol{\theta}})
$$

Estatistica da Razão de Verossimilhança

$$
\begin{gathered}
\text { - } Q_{L}=-2 \log \lambda=2[L(\hat{\boldsymbol{\theta}})-L(\overline{\boldsymbol{\theta}})] \\
e m q u e \lambda=\sup _{\left\{\boldsymbol{\theta}_{\epsilon \Theta: \boldsymbol{h}}(\boldsymbol{\theta})=0\right\}} L(\boldsymbol{\theta}) / \sup _{\left\{\boldsymbol{\theta}_{\epsilon \Theta\}}\right.} L(\boldsymbol{\theta}) .
\end{gathered}
$$

Estatistica Score

- $Q_{R}=n^{-1}[U(\overline{\boldsymbol{\theta}})]^{t}[I(\overline{\boldsymbol{\theta}})]^{-1}[U(\overline{\boldsymbol{\theta}})]$, 
em que $U(\overline{\boldsymbol{\theta}})=\left.U(\theta)\right|_{\theta=\bar{\theta}}$ e $U(\boldsymbol{\theta})$ como definido na Seção 2.1.

(II) Então $Q_{W}, Q_{L}, Q_{R}$ têm distribuição assintótica $\chi_{r}^{2}$ sob $H_{0}$. 


\section{Apêndice B}

Neste Apêndice apresentaremos os resultados da simulação do valor empírico dos testes de Wald e razão de verossimilhança para a distribuição Normal e t de Student, para 3 laboratórios e 5 rotações.

Tabela 25: Tamanhos empíricos dos testes de Wald e Razão de Verossimilhança para a primeira hipótese com $\mathrm{p}=3$ e $\mathrm{m}=5$ - Distribuição Normal.

\begin{tabular}{|c|c|c|c|c|c|c|c|c|c|c|c|}
\hline \multirow[b]{2}{*}{ nivel } & \multirow[b]{2}{*}{ teste } & \multirow[b]{2}{*}{ ni } & \multicolumn{3}{|c|}{$\sigma_{x_{j s}}^{2}$} & \multicolumn{3}{|c|}{$\sigma_{x_{j}}^{2}$} & \multicolumn{3}{|c|}{$\sigma_{x_{i l}}^{2}$} \\
\hline & & & $\sigma_{s}^{2}$ & $\sigma^{2}$ & $\sigma_{l}^{2}$ & $\sigma_{s}^{2}$ & $\sigma^{2}$ & $\sigma_{l}^{2}$ & $\sigma_{s}^{2}$ & $\sigma^{2}$ & $\sigma_{l}^{2}$ \\
\hline \multirow[t]{6}{*}{0,01} & W & 3 & 0,0092 & 0,0094 & 0,0096 & 0,0088 & 0,0089 & 0,0092 & 0,0096 & 0,0092 & 0,0093 \\
\hline & W & 5 & 0,0097 & 0,0099 & 0,0099 & 0,0096 & 0,0094 & 0,0098 & 0,0099 & 0,0098 & 0,0098 \\
\hline & W & 20 & 0,0099 & 0,010 & 0,010 & 0,010 & 0,0099 & 0,010 & 0,010 & 0,010 & 0,010 \\
\hline & RV & 3 & 0,0090 & 0,0092 & 0,0090 & 0,0092 & 0,0084 & 0,0084 & 0,0093 & 0,0090 & 0,0092 \\
\hline & RV & 5 & 0,0094 & 0,0096 & 0,0093 & 0,0096 & 0,0088 & 0,0088 & 0,0095 & 0,0094 & 0,0096 \\
\hline & RV & 20 & 0,0098 & 0,0099 & 0,0099 & 0,0099 & 0,0098 & 0,0099 & 0,0099 & 0,0098 & 0,0099 \\
\hline \multirow[t]{6}{*}{0,05} & W & 3 & 0,0472 & 0,0478 & 0,0484 & 0,0484 & 0,0478 & 0,0488 & 0,0489 & 0,049 & 0,0488 \\
\hline & W & 5 & 0,0488 & 0,0486 & 0,049 & 0,0496 & 0,0482 & 0,0492 & 0,0492 & 0,0499 & 0,0496 \\
\hline & W & 20 & 0,0499 & 0,0497 & 0,050 & 0,050 & 0,0498 & 0,050 & 0,0499 & 0,050 & 0,0499 \\
\hline & RV & 3 & 0,0471 & 0,0484 & 0,0480 & 0,0472 & 0,0463 & 0,0488 & 0,0478 & 0,0494 & 0,0484 \\
\hline & RV & 5 & 0,0490 & 0,0492 & 0,0489 & 0,0484 & 0,0488 & 0,0496 & 0,0489 & 0,0496 & 0,0499 \\
\hline & RV & 20 & 0,0499 & 0,0499 & 0,0499 & 0,0499 & 0,0498 & 0,050 & 0,0499 & 0,0499 & 0,05 \\
\hline \multirow[t]{6}{*}{0,1} & W & 3 & 0,086 & 0,096 & 0,0988 & 0,090 & 0,0944 & 0,0954 & 0,088 & 0,090 & 0,093 \\
\hline & W & 5 & 0,097 & 0,099 & 0,0999 & 0,0981 & 0,0963 & 0,0966 & 0,098 & 0,098 & 0,096 \\
\hline & W & 20 & 0,10 & 0,10 & 0,10 & 0,099 & 0,0999 & 0,0999 & 0,10 & 0,10 & 0,099 \\
\hline & RV & 3 & & 0,093 & & 0,089 & 0,0931 & & 0,090 & 0,089 & 0,090 \\
\hline & RV & 5 & 0,098 & 0,098 & 0,0989 & 0,0974 & 0,0967 & 0,0976 & 0,096 & 0,096 & 0,094 \\
\hline & RV & 20 & 0,099 & 0,10 & 0,0998 & 0,10 & 0,0989 & 0,0999 & 0,099 & 0,0999 & 0,0999 \\
\hline
\end{tabular}


Tabela 26: Tamanhos empíricos dos testes de Wald e Razão de Verossimilhança para a segunda hipótese com $\mathrm{p}=3$ e $\mathrm{m}=5$ - Distribuição Normal.

\begin{tabular}{|c|c|c|c|c|c|c|c|c|c|c|c|}
\hline \multirow[b]{2}{*}{ nivel } & \multirow[b]{2}{*}{ teste } & \multirow[b]{2}{*}{ ni } & \multicolumn{3}{|c|}{$\sigma_{x_{j s}}^{2}$} & \multicolumn{3}{|c|}{$\sigma_{x}^{2}$} & \multicolumn{3}{|c|}{$\sigma_{x_{j l}}^{2}$} \\
\hline & & & $\sigma_{s}^{2}$ & $\begin{array}{r}\sigma_{j} \\
\sigma^{2}\end{array}$ & $\sigma_{l}^{2}$ & $\sigma_{s}^{2}$ & $\sigma^{2}$ & $\sigma_{l}^{2}$ & $\sigma_{s}^{2}$ & $\sigma^{2}$ & $\sigma_{l}^{2}$ \\
\hline \multirow[t]{6}{*}{0,01} & W & 3 & 0,0082 & 0,0086 & 0,0092 & 0,0089 & 0,0093 & 0,0090 & 0,0079 & 0,0088 & 0,0094 \\
\hline & W & 5 & 0,0086 & 0,0094 & 0,0098 & 0,0092 & 0,0096 & 0,0098 & 0,0088 & 0,0097 & 0,0096 \\
\hline & W & 20 & 0,0096 & 0,0099 & 0,010 & 0,0099 & 0,0098 & 0,10 & 0,0098 & 0,0099 & 0,010 \\
\hline & RV & 3 & 0,0080 & 0,0084 & 0,0090 & 0,0088 & 0,0092 & 0,0088 & 0,0088 & 0,0082 & 0,0088 \\
\hline & $\mathrm{RV}$ & 5 & 0,0085 & 0,0092 & 0,0092 & 0,0091 & 0,0096 & 0,0094 & 0,009 & 0,0093 & 0,0091 \\
\hline & $\mathrm{RV}$ & 20 & 0,0098 & 0,0099 & 0,0099 & 0,0099 & 0,0099 & 0,0099 & 0,01 & 0,0099 & 0,0096 \\
\hline \multirow[t]{6}{*}{0,05} & W & 3 & 0,0471 & 0,0472 & 0,0479 & 0,0477 & 0,0484 & 0,0492 & 0,048 & 0,048 & 0,0488 \\
\hline & W & 5 & 0,0482 & 0,0482 & 0,0488 & 0,0483 & 0,0488 & 0,0496 & 0,0492 & 0,049 & 0,0499 \\
\hline & W & 20 & 0,0499 & 0,0499 & 0,0498 & 0,0499 & 0,0492 & 0,4998 & 0,0497 & 0,050 & 0,050 \\
\hline & $\mathrm{RV}$ & 3 & 0,0470 & 0,0474 & 0,0472 & 0,0462 & 0,0485 & 0,0484 & 0,046 & 0,0484 & 0,0474 \\
\hline & RV & 5 & 0,0487 & 0,0484 & 0,0484 & 0,0479 & 0,0489 & 0,0491 & 0,0484 & 0,0492 & 0,0496 \\
\hline & $\mathrm{RV}$ & 20 & 0,0499 & 0,0485 & 0,0499 & 0,0489 & 0,0497 & 0,0499 & 0,0499 & 0,0498 & 0,0499 \\
\hline \multirow[t]{6}{*}{0,1} & W & 3 & 0,0978 & 0,0944 & 0,0955 & 0,0963 & 0,0914 & 0,091 & 0,080 & 0,0991 & 0,0994 \\
\hline & W & 5 & 0,098 & 0,0978 & 0,0984 & 0,0989 & 0,099 & 0,0938 & 0,087 & 0,0994 & 0,0996 \\
\hline & W & 20 & 0,10 & 0,0992 & 0,0999 & 0,0999 & 0,10 & 0,10 & 0,098 & 0,0999 & 0,10 \\
\hline & $\mathrm{RV}$ & 3 & 0,0966 & 0,0948 & 0,0951 & 0,0951 & 0,0901 & 0,090 & 0,081 & 0,0928 & 0,088 \\
\hline & $\mathrm{RV}$ & 5 & 0,099 & 0,0974 & 0,099 & 0,0987 & 0,099 & 0,0948 & 0,0844 & 0,0978 & 0,098 \\
\hline & RV & 20 & 0,10 & 0,0989 & 0,10 & 0,099 & 0,10 & 0,099 & 0,096 & 0,0989 & 0,10 \\
\hline
\end{tabular}


Tabela 27: Tamanhos empíricos dos testes de Wald e Razão de Verossimilhança para a terceira hipótese com $\mathrm{p}=3$ e $\mathrm{m}=5$ - Distribuição Normal.

\begin{tabular}{|c|c|c|c|c|c|c|c|c|c|c|c|}
\hline \multirow[b]{2}{*}{ nivel } & \multirow[b]{2}{*}{ teste } & \multirow[b]{2}{*}{ ni } & \multicolumn{3}{|c|}{$\sigma_{x_{j s}}^{2}$} & \multicolumn{3}{|c|}{$\sigma_{x}^{2}$} & \multicolumn{3}{|c|}{$\sigma_{x_{i l}}^{2}$} \\
\hline & & & $\sigma_{s}^{2}$ & $\begin{array}{r}\sigma_{j} \\
\sigma^{2}\end{array}$ & $\sigma_{l}^{2}$ & $\sigma_{s}^{2}$ & $\sigma^{2}$ & $\sigma_{l}^{2}$ & $\sigma_{s}^{2}$ & $\sigma^{2}$ & $\sigma_{l}^{2}$ \\
\hline \multirow[t]{6}{*}{0,01} & W & 3 & 0,0094 & 0,0086 & 0,0089 & 0,0088 & 0,0092 & 0,0090 & 0,0094 & 0,0088 & 0,0086 \\
\hline & W & 5 & 0,0098 & 0,0088 & 0,0094 & 0,0096 & 0,0098 & 0,0096 & 0,0099 & 0,0096 & 0,0094 \\
\hline & W & 20 & 0,010 & 0,0094 & 0,0099 & 0,010 & 0,0099 & 0,010 & 0,010 & 0,010 & 0,010 \\
\hline & RV & 3 & 0,0092 & 0,0082 & 0,0084 & 0,0084 & 0,0090 & 0,0089 & 0,0090 & 0,0086 & 0,0084 \\
\hline & $\mathrm{RV}$ & 5 & 0,0095 & 0,0086 & 0,0093 & 0,0088 & 0,0092 & 0,0093 & 0,0092 & 0,0094 & 0,0092 \\
\hline & $\mathrm{RV}$ & 20 & 0,0099 & 0,0092 & 0,0099 & 0,0099 & 0,0098 & 0,0098 & 0,0098 & 0,0099 & 0,0098 \\
\hline \multirow[t]{6}{*}{0,05} & W & 3 & 0,0489 & 0,046 & 0,0476 & 0,0472 & 0,0484 & 0,0477 & 0,0486 & 0,0476 & 0,0485 \\
\hline & W & 5 & 0,0491 & 0,0489 & 0,0488 & 0,0484 & 0,0498 & 0,0481 & 0,0499 & 0,0486 & 0,0493 \\
\hline & W & 20 & 0,0499 & 0,0496 & 0,0499 & 0,0499 & 0,050 & 0,0499 & 0,050 & 0,0499 & 0,0498 \\
\hline & $\mathrm{RV}$ & 3 & 0,0478 & 0,0474 & 0,0463 & 0,0482 & 0,0480 & 0,0474 & 0,0472 & 0,0454 & 0,0487 \\
\hline & RV & 5 & 0,0489 & 0,0482 & 0,0479 & 0,0488 & 0,0490 & 0,0483 & 0,0486 & 0,0492 & 0,0492 \\
\hline & $\mathrm{RV}$ & 20 & 0,0499 & 0,0499 & 0,0499 & 0,0498 & 0,0499 & 0,0498 & 0,0499 & 0,0498 & 0,0494 \\
\hline \multirow[t]{6}{*}{0,1} & W & 3 & 0,0969 & 0,0963 & 0,0966 & 0,0984 & 0,0986 & 0,0962 & 0,0974 & 0,0944 & 0,089 \\
\hline & W & 5 & 0,0978 & 0,0976 & 0,0992 & 0,0986 & 0,0998 & 0,0986 & 0,0980 & 0,0952 & 0,094 \\
\hline & W & 20 & 0,0999 & 0,0999 & 0,10 & 0,099 & 0,10 & 0,099 & 0,099 & 0,0999 & 0,10 \\
\hline & $\mathrm{RV}$ & 3 & 0,0964 & 0,0961 & 0,0956 & 0,0972 & 0,0973 & 0,0961 & 0,0961 & 0,094 & 0,088 \\
\hline & $\mathrm{RV}$ & 5 & 0,0971 & 0,0974 & 0,0988 & 0,0996 & 0,0988 & 0,0974 & 0,0979 & 0,095 & 0,094 \\
\hline & $\mathrm{RV}$ & 20 & 0,0989 & 0,0989 & 0,099 & 0,10 & 0,0999 & 0,0999 & 0,099 & 0,0984 & 0,099 \\
\hline
\end{tabular}


Tabela 28: Tamanhos empíricos dos testes de Wald e Razão de Verossimilhança para a quarta hipótese com $\mathrm{p}=3$ e $\mathrm{m}=5$ - Distribuição Normal.

\begin{tabular}{|c|c|c|c|c|c|c|c|c|c|c|c|}
\hline \multirow[b]{2}{*}{ nivel } & \multirow[b]{2}{*}{ teste } & \multirow[b]{2}{*}{ ni } & \multicolumn{3}{|c|}{$\sigma_{x_{i s}}^{2}$} & \multicolumn{3}{|c|}{$\sigma_{x}^{2}$} & \multicolumn{3}{|c|}{$\sigma_{x_{i l}}^{2}$} \\
\hline & & & $\sigma_{s}^{2}$ & $\begin{array}{r}x_{j s} \\
\sigma^{2}\end{array}$ & $\sigma_{l}^{2}$ & $\sigma_{s}^{2}$ & $\sigma_{j}^{2}$ & $\sigma_{l}^{2}$ & $\sigma_{s}^{2}$ & $\sigma_{j l}^{x_{j}} \sigma^{2}$ & $\sigma_{l}^{2}$ \\
\hline \multirow[t]{6}{*}{0,01} & $\mathrm{~W}$ & 3 & 0,0086 & 0,0082 & 0,0094 & 0,0086 & 0,0086 & 0,0092 & 0,0082 & 0,0084 & 0,0079 \\
\hline & W & 5 & 0,0094 & 0,0094 & 0,0098 & 0,0098 & 0,0098 & 0,0096 & 0,0092 & 0,0096 & 0,0086 \\
\hline & W & 20 & 0,0099 & 0,0099 & 0,10 & 0,010 & 0,010 & 0,0099 & 0,0099 & 0,010 & 0,0099 \\
\hline & RV & 3 & 0,0082 & 0,0084 & 0,0092 & 0,0096 & 0,0082 & 0,0090 & 0,0088 & 0,008 & 0,0080 \\
\hline & RV & 5 & 0,0091 & 0,0092 & 0,0094 & 0,0098 & 0,0086 & 0,0094 & 0,0098 & 0,0092 & 0,0091 \\
\hline & RV & 20 & 0,0098 & 0,010 & 0,0098 & 0,010 & 0,0099 & 0,0097 & 0,010 & 0,0098 & 0,0096 \\
\hline \multirow[t]{6}{*}{0,05} & W & 3 & 0,0468 & 0,0486 & 0,0477 & 0,0487 & 0,0484 & 0,0474 & 0,046 & 0,0476 & 0,0484 \\
\hline & W & 5 & 0,0489 & 0,0498 & 0,0489 & 0,0498 & 0,0498 & 0,0498 & 0,048 & 0,0481 & 0,0488 \\
\hline & W & 20 & 0,0499 & 0,050 & 0,0499 & 0,050 & 0,050 & 0,050 & 0,0499 & 0,0499 & 0,0493 \\
\hline & RV & 3 & 0,0467 & 0,0488 & 0,0470 & 0,0484 & 0,0480 & 0,047 & 0,0468 & 0,047 & 0,048 \\
\hline & RV & 5 & 0,0484 & 0,0499 & 0,0483 & 0,0496 & 0,0499 & 0,0484 & 0,0477 & 0,0479 & 0,0488 \\
\hline & RV & 20 & 0,0499 & 0,0500 & 0,0498 & 0,0499 & 0,050 & 0,0498 & 0,0498 & 0,0489 & 0,0493 \\
\hline \multirow[t]{6}{*}{0,1} & W & 3 & 0,090 & 0,082 & 0,086 & 0,0979 & 0,0982 & 0,092 & 0,082 & 0,094 & 0,086 \\
\hline & W & 5 & 0,096 & 0,094 & 0,0924 & 0,0985 & 0,0996 & 0,096 & 0,092 & 0,0979 & 0,096 \\
\hline & W & 20 & 0,099 & 0,096 & 0,099 & 0,099 & 0,10 & 0,099 & 0,0999 & 0,10 & 0,10 \\
\hline & RV & 3 & 0,089 & 0,0848 & 0,088 & 0,0976 & 0,0978 & 0,093 & 0,080 & 0,092 & 0,086 \\
\hline & RV & 5 & 0,092 & 0,0968 & 0,0992 & 0,0982 & 0,0988 & 0,0956 & 0,091 & 0,098 & 0,098 \\
\hline & RV & 20 & 0,099 & 0,099 & 0,10 & 0,099 & 0,099 & 0,099 & 0,099 & 0,10 & 0,099 \\
\hline
\end{tabular}


Tabela 29: Tamanhos empíricos dos testes de Wald e Razão de Verossimilhança para a quinta hipótese com $\mathrm{p}=3$ e $\mathrm{m}=5$ - Distribuição Normal.

\begin{tabular}{|c|c|c|c|c|c|c|c|c|c|c|c|}
\hline \multirow[b]{2}{*}{ nivel } & \multirow[b]{2}{*}{ teste } & \multirow[b]{2}{*}{ ni } & \multicolumn{3}{|c|}{$\sigma_{x_{i s}}^{2}$} & \multicolumn{3}{|c|}{$\sigma_{x_{i}}^{2}$} & \multicolumn{3}{|c|}{$\sigma_{x_{j i}}^{2}$} \\
\hline & & & $\sigma_{s}^{2}$ & $\begin{array}{r}x_{j s} \\
\sigma^{2}\end{array}$ & $\sigma_{l}^{2}$ & $\sigma_{s}^{2}$ & $\sigma_{j}^{2}$ & $\sigma_{l}^{2}$ & $\sigma_{s}^{2}$ & $\sigma_{j l}^{x_{j}} \sigma^{2}$ & $\sigma_{l}^{2}$ \\
\hline \multirow[t]{6}{*}{0,01} & $\mathrm{~W}$ & 3 & 0,0096 & 0,0092 & 0,0088 & 0,0092 & 0,0094 & 0,0082 & 0,0088 & 0,0084 & 0,0096 \\
\hline & W & 5 & 0,0099 & 0,0098 & 0,0096 & 0,0096 & 0,0096 & 0,0089 & 0,0099 & 0,0086 & 0,0099 \\
\hline & W & 20 & 0,010 & 0,010 & 0,0099 & 0,0099 & 0,0099 & 0,0099 & 0,010 & 0,0098 & 0,10 \\
\hline & RV & 3 & 0,0094 & 0,0089 & 0,0082 & 0,0091 & 0,0091 & 0,0079 & 0,0083 & 0,0084 & 0,0098 \\
\hline & RV & 5 & 0,0097 & 0,0096 & 0,0094 & 0,0096 & 0,0096 & 0,0084 & 0,0092 & 0,0088 & 0,0099 \\
\hline & RV & 20 & 0,0099 & 0,0099 & 0,0098 & 0,0099 & 0,0099 & 0,10 & 0,0099 & 0,0099 & 0,10 \\
\hline \multirow[t]{6}{*}{0,05} & W & 3 & 0,0478 & 0,0473 & 0,0452 & 0,0475 & 0,0487 & 0,0474 & 0,0488 & 0,046 & 0,0448 \\
\hline & W & 5 & 0,0482 & 0,4862 & 0,0486 & 0,0484 & 0,0498 & 0,0488 & 0,0498 & 0,0482 & 0,0489 \\
\hline & W & 20 & 0,0499 & 0,0499 & 0,0499 & 0,0499 & 0,050 & 0,0499 & 0,050 & 0,0496 & 0,500 \\
\hline & RV & 3 & 0,0478 & 0,0478 & 0,0454 & 0,0471 & 0,0481 & 0,047 & 0,0489 & 0,0466 & 0,0442 \\
\hline & RV & 5 & 0,0484 & 0,0487 & 0,0484 & 0,0484 & 0,049 & 0,0488 & 0,0496 & 0,0486 & 0,0484 \\
\hline & RV & 20 & 0,0499 & 0,050 & 0,0491 & 0,0498 & 0,0499 & 0,050 & 0,0499 & 0,050 & 0,0498 \\
\hline \multirow[t]{6}{*}{0,1} & W & 3 & 0,0968 & 0,094 & 0,0846 & 0,089 & 0,0996 & 0,0978 & 0,0955 & 0,093 & 0,093 \\
\hline & W & 5 & 0,0988 & 0,098 & 0,0896 & 0,0998 & 0,0999 & 0,0996 & 0,0988 & 0,096 & 0,0992 \\
\hline & W & 20 & 0,10 & 0,10 & 0,0998 & 0,10 & 0,10 & 0,10 & 0,099 & 0,0988 & 0,10 \\
\hline & RV & 3 & 0,0943 & 0,090 & 0,0831 & 0,087 & 0,098 & 0,0978 & 0,0955 & 0,091 & 0,088 \\
\hline & RV & 5 & 0,0968 & 0,093 & 0,0898 & 0,096 & 0,0999 & 0,0998 & 0,0972 & 0,098 & 0,097 \\
\hline & RV & 20 & 0,099 & 0,099 & 0,099 & 0,099 & 0,10 & 0,10 & 0,10 & 0,099 & 0,099 \\
\hline
\end{tabular}


Tabela 30: Tamanhos empíricos dos testes de Wald e Razão de Verossimilhança para a sexta hipótese com $\mathrm{p}=3$ e $\mathrm{m}=5$ - Distribuição Normal.

\begin{tabular}{|c|c|c|c|c|c|c|c|c|c|c|c|}
\hline \multirow[b]{2}{*}{ nivel } & \multirow[b]{2}{*}{ teste } & \multirow[b]{2}{*}{ ni } & \multicolumn{3}{|c|}{$\sigma_{x_{i s}}^{2}$} & \multicolumn{3}{|c|}{$\sigma_{x_{j}}^{2}$} & \multicolumn{3}{|c|}{$\sigma_{x_{i l}}^{2}$} \\
\hline & & & $\sigma_{s}^{2}$ & $\sigma^{2}$ & $\sigma_{l}^{2}$ & $\sigma_{s}^{2}$ & $\sigma^{2}$ & $\sigma_{l}^{2}$ & $\sigma_{s}^{2}$ & $\sigma^{2}$ & $\sigma_{l}^{2}$ \\
\hline \multirow[t]{6}{*}{0,01} & W & 3 & 0,0093 & 0,0094 & 0,0094 & 0,0082 & 0,0089 & 0,0087 & 0,0084 & 0,0088 & 0,0092 \\
\hline & W & 5 & 0,0096 & 0,0098 & 0,0098 & 0,0097 & 0,0092 & 0,0092 & 0,0092 & 0,0094 & 0,0099 \\
\hline & W & 20 & 0,0099 & 0,010 & 0,010 & 0,0099 & 0,0099 & 0,0099 & 0,0098 & 0,0099 & 0,010 \\
\hline & RV & 3 & 0,0091 & 0,0092 & 0,0090 & 0,0081 & 0,0080 & 0,0089 & 0,0082 & 0,0084 & 0,0089 \\
\hline & RV & 5 & 0,0094 & 0,0096 & 0,0094 & 0,0094 & 0,0086 & 0,0094 & 0,0096 & 0,0091 & 0,0093 \\
\hline & RV & 20 & 0,0097 & 0,0099 & 0,0099 & 0,0098 & 0,099 & 0,010 & 0,0099 & 0,0099 & 0,0099 \\
\hline \multirow[t]{6}{*}{0,05} & W & 3 & 0,0484 & 0,0485 & 0,0498 & 0,0472 & 0,0476 & 0,0479 & 0,0475 & 0,0473 & 0,0459 \\
\hline & W & 5 & 0,0497 & 0,0492 & 0,0499 & 0,0484 & 0,049 & 0,0488 & 0,0487 & 0,0482 & 0,0472 \\
\hline & W & 20 & 0,50 & 0,0499 & 0,050 & 0,0498 & 0,050 & 0,050 & 0,0499 & 0,0499 & 0,0499 \\
\hline & RV & 3 & 0,0480 & 0,0454 & 0,0492 & 0,0470 & 0,0489 & 0,0486 & 0,0476 & 0,0471 & 0,0456 \\
\hline & RV & 5 & 0,0489 & 0,0468 & 0,0498 & 0,0482 & 0,0494 & 0,0492 & 0,0488 & 0,0484 & 0,0488 \\
\hline & RV & 20 & 0,0499 & 0,0499 & 0,0499 & 0,0489 & 0,0499 & 0,050 & 0,0498 & 0,050 & 0,0498 \\
\hline \multirow[t]{6}{*}{0,1} & W & 3 & 0,0884 & 0,094 & 0,0898 & 0,089 & 0,096 & 0,0976 & 0,084 & 0,0934 & 0,092 \\
\hline & W & 5 & 0,0987 & 0,098 & 0,098 & 0,0952 & 0,0978 & 0,0988 & 0,096 & 0,0966 & 0,099 \\
\hline & W & 20 & 0,10 & 0,10 & 0,10 & 0,0999 & 0,0998 & 0,099 & 0,10 & 0,0999 & 0,10 \\
\hline & RV & 3 & 0,0849 & 0,090 & 0,0848 & 0,090 & 0,0949 & 0,0961 & 0,081 & 0,0921 & 0,089 \\
\hline & RV & 5 & 0,0983 & 0,096 & 0,0964 & 0,0958 & 0,0962 & 0,0984 & 0,093 & 0,0946 & 0,093 \\
\hline & RV & 20 & 0,0999 & 0,099 & 0,0998 & 0,0999 & 0,099 & 0,10 & 0,099 & 0,099 & 0,099 \\
\hline
\end{tabular}


Tabela 31: Tamanhos empíricos dos testes de Wald e Razão de Verossimilhança para a primeira hipótese com $\mathrm{p}=3$ e $\mathrm{m}=5$ - Distribuição t de Student.

\begin{tabular}{|c|c|c|c|c|c|c|c|c|c|c|c|}
\hline \multirow[b]{2}{*}{ nivel } & \multirow[b]{2}{*}{ teste } & \multirow[b]{2}{*}{ ni } & \multicolumn{3}{|c|}{$\sigma_{x_{i s}}^{2}$} & \multicolumn{3}{|c|}{$\sigma_{x_{i}}^{2}$} & \multicolumn{3}{|c|}{$\sigma_{x_{i l}}^{2}$} \\
\hline & & & $\sigma_{s}^{2}$ & $\begin{array}{r}x_{j s} \\
\sigma^{2}\end{array}$ & $\sigma_{l}^{2}$ & $\sigma_{s}^{2}$ & $\sigma_{j}^{2}$ & $\sigma_{l}^{2}$ & $\sigma_{s}^{2}$ & $\sigma^{2}$ & $\sigma_{l}^{2}$ \\
\hline \multirow[t]{6}{*}{0,01} & $\mathrm{~W}$ & 3 & 0,0115 & 0,0102 & 0,0118 & 0,0119 & 0,0109 & 0,0116 & 0,0104 & 0,0114 & 0,011 \\
\hline & W & 5 & 0,0112 & 0,0101 & 0,0116 & 0,0116 & 0,0106 & 0,0110 & 0,0103 & 0,0111 & 0,0103 \\
\hline & W & 20 & 0,0106 & 0,010 & 0,0101 & 0,010 & 0,0102 & 0,0101 & 0,0100 & 0,0102 & 0,0101 \\
\hline & RV & 3 & 0,0113 & 0,0101 & 0,012 & 0,012 & 0,0111 & 0,0113 & 0,0104 & 0,0116 & 0,0112 \\
\hline & RV & 5 & 0,011 & 0,0100 & 0,0101 & 0,0118 & 0,011 & 0,0102 & 0,0104 & 0,0110 & 0,0102 \\
\hline & RV & 20 & 0,0101 & 0,010 & 0,10 & 0,0100 & 0,010 & 0,010 & 0,1001 & 0,0101 & 0,0100 \\
\hline \multirow[t]{6}{*}{0,05} & W & 3 & 0,0511 & 0,0509 & 0,0510 & 0,052 & 0,0512 & 0,0514 & 0,0504 & 0,0512 & 0,0511 \\
\hline & W & 5 & 0,0504 & 0,0504 & 0,0504 & 0,0514 & 0,0510 & 0,0502 & 0,0502 & 0,0506 & 0,051 \\
\hline & W & 20 & 0,0501 & 0,0501 & 0,05 & 0,0500 & 0,05 & 0,0500 & 0,050 & 0,0501 & 0,050 \\
\hline & RV & 3 & 0,0511 & 0,0504 & 0,0514 & 0,0519 & 0,0518 & 0,0515 & 0,0503 & 0,051 & 0,0521 \\
\hline & RV & 5 & 0,0502 & 0,0502 & 0,0508 & 0,0506 & 0,0506 & 0,0503 & 0,0502 & 0,0502 & 0,0512 \\
\hline & RV & 20 & 0,0500 & 0,0500 & 0,0502 & 0,0500 & 0,0500 & 0,0500 & 0,050 & 0,0500 & 0,0500 \\
\hline \multirow[t]{6}{*}{0,10} & W & 3 & 0,112 & 0,110 & 0,1041 & 0,1056 & 0,112 & 0,1042 & 0,11 & 0,1034 & 0,1011 \\
\hline & W & 5 & 0,1045 & 0,102 & 0,1038 & 0,1032 & 0,111 & 0,1028 & 0,104 & 0,1012 & 0,1013 \\
\hline & W & 20 & 0,1016 & 0,10 & 0,1014 & 0,101 & 0,10 & 0,1008 & 0,1002 & 0,1004 & 0,1007 \\
\hline & RV & 3 & 0,11 & 0,118 & 0,1023 & 0,1055 & 0,11 & 0,1026 & 0,116 & 0,1029 & 0,1020 \\
\hline & RV & 5 & 0,1023 & 0,109 & 0,101 & 0,1038 & 0,1008 & 0,1012 & 0,1048 & 0,1012 & 0,1013 \\
\hline & RV & 20 & 0,1011 & 0,1004 & 0,10 & 0,1005 & 0,10 & 0,1003 & 0,1021 & 0,10 & 0,1004 \\
\hline
\end{tabular}


Tabela 32: Tamanhos empíricos dos testes de Wald e Razão de Verossimilhança para a segunda hipótese com $\mathrm{p}=3$ e $\mathrm{m}=5$ - Distribuição t de Student.

\begin{tabular}{|c|c|c|c|c|c|c|c|c|c|c|c|}
\hline \multirow[b]{2}{*}{ nivel } & \multirow[b]{2}{*}{ teste } & \multirow[b]{2}{*}{ ni } & \multicolumn{3}{|c|}{$\sigma_{x_{i s}}^{2}$} & \multicolumn{3}{|c|}{$\sigma_{x}^{2}$} & \multicolumn{3}{|c|}{$\sigma_{x_{i l}}^{2}$} \\
\hline & & & $\sigma_{s}^{2}$ & $\begin{array}{r}x_{j s} \\
\sigma^{2}\end{array}$ & $\sigma_{l}^{2}$ & $\sigma_{s}^{2}$ & $\sigma_{j}^{2}$ & $\sigma_{l}^{2}$ & $\sigma_{s}^{2}$ & $\sigma^{2}$ & $\sigma_{l}^{2}$ \\
\hline \multirow[t]{6}{*}{0,01} & W & 3 & 0,0112 & 0,0114 & 0,011 & 0,0117 & 0,0112 & 0,0116 & 0,011 & 0,0113 & 0,0114 \\
\hline & W & 5 & 0,0106 & 0,0108 & 0,0108 & 0,0102 & 0,0108 & 0,0102 & 0,0108 & 0,0106 & 0,0104 \\
\hline & W & 20 & 0,0100 & 0,0100 & 0,0100 & 0,010 & 0,0100 & 0,010 & 0,0100 & 0,0100 & 0,010 \\
\hline & RV & 3 & 0,0102 & 0,0103 & 0,0102 & 0,0101 & 0,0102 & 0,011 & 0,011 & 0,0117 & 0,0106 \\
\hline & RV & 5 & 0,0101 & 0,0101 & 0,0101 & 0,0101 & 0,0102 & 0,0108 & 0,0105 & 0,0104 & 0,0103 \\
\hline & $\mathrm{RV}$ & 20 & 0,010 & 0,0100 & 0,010 & 0,010 & 0,0100 & 0,10 & 0,0101 & 0,0101 & 0,0101 \\
\hline \multirow[t]{6}{*}{0,05} & W & 3 & 0,0512 & 0,0512 & 0,0522 & 0,0513 & 0,0514 & 0,0527 & 0,0512 & 0,0513 & 0,051 \\
\hline & W & 5 & 0,0503 & 0,0501 & 0,0514 & 0,0506 & 0,0502 & 0,0516 & 0,0508 & 0,0502 & 0,0508 \\
\hline & W & 20 & 0,50 & 0,50 & 0,0500 & 0,0500 & 0,050 & 0,0500 & 0,05 & 0,0500 & 0,0500 \\
\hline & $\mathrm{RV}$ & 3 & 0,0524 & 0,0528 & 0,0516 & 0,0511 & 0,0514 & 0,051 & 0,0510 & 0,0512 & 0,0504 \\
\hline & $\mathrm{RV}$ & 5 & 0,0518 & 0,0516 & 0,0508 & 0,0508 & 0,0507 & 0,0508 & 0,0504 & 0,0511 & 0,0501 \\
\hline & $\mathrm{RV}$ & 20 & 0,0501 & 0,0501 & 0,0501 & 0,0500 & 0,0500 & 0,050 & 0,050 & 0,0504 & 0,050 \\
\hline \multirow[t]{6}{*}{0,10} & W & 3 & 0,1036 & 0,113 & 0,1035 & 0,1011 & 0,1028 & 0,11 & 0,1031 & 0,1012 & 0,1024 \\
\hline & W & 5 & 0,1002 & 0,108 & 0,1026 & 0,1008 & 0,1002 & 0,1024 & 0,1006 & 0,1006 & 0,1011 \\
\hline & W & 20 & 0,10 & 0,1007 & 0,1006 & 0,10 & 0,10 & 0,1015 & 0,1004 & 0,10 & 0,1004 \\
\hline & $\mathrm{RV}$ & 3 & 0,1034 & 0,11 & 0,1033 & 0,1089 & 0,110 & 0,121 & 0,1021 & 0,112 & 0,1062 \\
\hline & $\mathrm{RV}$ & 5 & 0,1014 & 0,1009 & 0,1024 & 0,1026 & 0,1006 & 0,1022 & 0,102 & 0,106 & 0,1041 \\
\hline & $\mathrm{RV}$ & 20 & 0,10 & 0,10 & 0,1004 & 0,1001 & 0,10 & 0,1011 & 0,1001 & 0,1004 & 0,1006 \\
\hline
\end{tabular}


Tabela 33: Tamanhos empíricos dos testes de Wald e Razão de Verossimilhança para a terceira hipótese com $\mathrm{p}=3$ e $\mathrm{m}=5$ - Distribuição t de Student.

\begin{tabular}{|c|c|c|c|c|c|c|c|c|c|c|c|}
\hline \multirow[b]{2}{*}{ nivel } & \multirow[b]{2}{*}{ teste } & \multirow[b]{2}{*}{ ni } & \multicolumn{3}{|c|}{$\sigma_{x_{j s}}^{2}$} & \multicolumn{3}{|c|}{$\sigma_{x_{j}}^{2}$} & \multicolumn{3}{|c|}{$\sigma_{x_{j l}}^{2}$} \\
\hline & & & $\sigma_{s}^{2}$ & $\sigma^{2}$ & $\sigma_{l}^{2}$ & $\sigma_{s}^{2}$ & $\sigma^{2}$ & $\sigma_{l}^{2}$ & $\sigma_{s}^{2}$ & $\sigma^{2}$ & $\sigma_{l}^{2}$ \\
\hline \multirow[t]{6}{*}{0,01} & W & 3 & 0,011 & 0,0113 & 0,0113 & 0,0108 & 0,0112 & 0,0112 & 0,0102 & 0,0112 & 0,0121 \\
\hline & W & 5 & 0,0102 & 0,0112 & 0,0102 & 0,0100 & 0,011 & 0,0109 & 0,0101 & 0,0108 & 0,0114 \\
\hline & W & 20 & 0,0100 & 0,0102 & 0,0100 & 0,010 & 0,0101 & 0,0100 & 0,010 & 0,0101 & 0,0107 \\
\hline & RV & 3 & 0,0111 & 0,0116 & 0,0113 & 0,0112 & 0,0112 & 0,0119 & 0,0102 & 0,0122 & 0,0112 \\
\hline & RV & 5 & 0,0109 & 0,011 & 0,0102 & 0,0103 & 0,0102 & 0,0112 & 0,0100 & 0,0112 & 0,0102 \\
\hline & $\mathrm{RV}$ & 20 & 0,0101 & 0,0103 & 0,010 & 0,010 & 0,0100 & 0,0100 & 0,10 & 0,0108 & 0,0100 \\
\hline \multirow[t]{6}{*}{0,05} & W & 3 & 0,0513 & 0,0512 & 0,0514 & 0,0513 & 0,0513 & 0,0513 & 0,0515 & 0,0514 & 0,0513 \\
\hline & W & 5 & 0,0512 & 0,0502 & 0,0513 & 0,0502 & 0,0503 & 0,0502 & 0,0504 & 0,0508 & 0,0508 \\
\hline & W & 20 & 0,0502 & 0,050 & 0,0502 & 0,050 & 0,0500 & 0,050 & 0,0500 & 0,0500 & 0,0500 \\
\hline & $\mathrm{RV}$ & 3 & 0,0514 & 0,0513 & 0,0513 & 0,0511 & 0,0512 & 0,0513 & 0,0515 & 0,5116 & 0,051 \\
\hline & $\mathrm{RV}$ & 5 & 0,0504 & 0,0504 & 0,0508 & 0,0508 & 0,0502 & 0,0503 & 0,0504 & 0,0504 & 0,0508 \\
\hline & $\mathrm{RV}$ & 20 & 0,0500 & 0,0500 & 0,0500 & 0,0501 & 0,050 & 0,0500 & 0,0500 & 0,050 & 0,050 \\
\hline \multirow[t]{6}{*}{0,10} & W & 3 & 0,11 & 0,1018 & 0,1022 & 0,112 & 0,102 & 0,1014 & 0,1036 & 0,1022 & 0,0106 \\
\hline & W & 5 & 0,104 & 0,1006 & 0,102 & 0,103 & 0,1018 & 0,1008 & 0,101 & 0,1012 & 0,0102 \\
\hline & W & 20 & 0,1001 & 0,1003 & 0,101 & 0,1007 & 0,1004 & 0,10 & 0,10 & 0,10 & 0,0101 \\
\hline & $\mathrm{RV}$ & 3 & 0,112 & 0,1014 & 0,1025 & 0,1082 & 0,1034 & 0,1024 & 0,1021 & 0,1048 & 0,0102 \\
\hline & $\mathrm{RV}$ & 5 & 0,104 & 0,1012 & 0,1016 & 0,103 & 0,1018 & 0,1018 & 0,1018 & 0,1012 & 0,0100 \\
\hline & $\mathrm{RV}$ & 20 & 0,1001 & 0,1001 & 0,10 & 0,1003 & 0,1002 & 0,1001 & 0,1003 & 0,10 & 0,10 \\
\hline
\end{tabular}


Tabela 34: Tamanhos empíricos dos testes de Wald e Razão de Verossimilhança para a quarta hipótese com $\mathrm{p}=3$ e $\mathrm{m}=5$ - Distribuição t de Student.

\begin{tabular}{|c|c|c|c|c|c|c|c|c|c|c|c|}
\hline \multirow[b]{2}{*}{ nivel } & \multirow[b]{2}{*}{ teste } & \multirow[b]{2}{*}{ ni } & \multicolumn{3}{|c|}{$\sigma_{x_{i s}}^{2}$} & \multicolumn{3}{|c|}{$\sigma_{x}^{2}$} & \multicolumn{3}{|c|}{$\sigma_{x_{i l}}^{2}$} \\
\hline & & & $\sigma_{s}^{2}$ & $\begin{array}{r}x_{j s} \\
\sigma^{2}\end{array}$ & $\sigma_{l}^{2}$ & $\sigma_{s}^{2}$ & $\sigma_{j}^{2}$ & $\sigma_{l}^{2}$ & $\sigma_{s}^{2}$ & $\sigma_{j l}^{x_{j}} \sigma^{2}$ & $\sigma_{l}^{2}$ \\
\hline \multirow[t]{6}{*}{0,01} & $\mathrm{~W}$ & 3 & 0,0119 & 0,0102 & 0,0103 & 0,0118 & 0,0108 & 0,0105 & 0,0107 & 0,0108 & 0,0112 \\
\hline & W & 5 & 0,0109 & 0,0102 & 0,0103 & 0,0106 & 0,0106 & 0,0103 & 0,0103 & 0,0106 & 0,0108 \\
\hline & W & 20 & 0,0101 & 0,010 & 0,010 & 0,0100 & 0,0100 & 0,010 & 0,0102 & 0,0100 & 0,0100 \\
\hline & RV & 3 & 0,0117 & 0,0112 & 0,0103 & 0,0102 & 0,0104 & 0,0105 & 0,0109 & 0,0107 & 0,0113 \\
\hline & RV & 5 & 0,011 & 0,0108 & 0,0103 & 0,0102 & 0,0101 & 0,0102 & 0,0105 & 0,0106 & 0,0106 \\
\hline & RV & 20 & 0,010 & 0,0101 & 0,0100 & 0,0100 & 0,10 & 0,010 & 0,0101 & 0,0101 & 0,0100 \\
\hline \multirow[t]{6}{*}{0,05} & W & 3 & 0,0511 & 0,0513 & 0,0516 & 0,0515 & 0,0514 & 0,0512 & 0,0511 & 0,0512 & 0,0511 \\
\hline & W & 5 & 0,0511 & 0,0507 & 0,0515 & 0,0513 & 0,0511 & 0,0509 & 0,0511 & 0,0511 & 0,0510 \\
\hline & W & 20 & 0,0501 & 0,0500 & 0,0500 & 0,0501 & 0,0501 & 0,0501 & 0,0501 & 0,0501 & 0,0502 \\
\hline & RV & 3 & 0,0518 & 0,0511 & 0,0516 & 0,0515 & 0,0513 & 0,0511 & 0,0513 & 0,0512 & 0,0510 \\
\hline & RV & 5 & 0,0509 & 0,0503 & 0,0505 & 0,0512 & 0,0508 & 0,0508 & 0,0511 & 0,0510 & 0,0505 \\
\hline & RV & 20 & 0,0500 & 0,050 & 0,0501 & 0,0503 & 0,0501 & 0,0500 & 0,0500 & 0,0501 & 0,0500 \\
\hline \multirow[t]{6}{*}{0,10} & W & 3 & 0,1012 & 0,116 & 0,113 & 0,1072 & 0,1021 & 0,1076 & 0,1023 & 0,1026 & 0,1013 \\
\hline & W & 5 & 0,1011 & 0,107 & 0,107 & 0,1056 & 0,1018 & 0,104 & 0,101 & 0,1014 & 0,1007 \\
\hline & W & 20 & 0,10 & 0,1004 & 0,1009 & 0,1004 & 0,10 & 0,1002 & 0,10 & 0,1004 & 0,10 \\
\hline & RV & 3 & 0,1013 & 0,117 & 0,11 & 0,1094 & 0,1022 & 0,1065 & 0,11 & 0,1063 & 0,101 \\
\hline & RV & 5 & 0,1011 & 0,109 & 0,1082 & 0,1074 & 0,101 & 0,101 & 0,1031 & 0,1024 & 0,1008 \\
\hline & RV & 20 & 0,10 & 0,1001 & 0,1002 & 0,1004 & 0,10 & 0,1007 & 0,1009 & 0,1006 & 0,10 \\
\hline
\end{tabular}


Tabela 35: Tamanhos empíricos dos testes de Wald e Razão de Verossimilhança para a quinta hipótese com $\mathrm{p}=3$ e $\mathrm{m}=5$ - Distribuição t de Student.

\begin{tabular}{|c|c|c|c|c|c|c|c|c|c|c|c|}
\hline \multirow[b]{2}{*}{ nivel } & \multirow[b]{2}{*}{ teste } & \multirow[b]{2}{*}{ ni } & \multicolumn{3}{|c|}{$\sigma_{x_{i s}}^{2}$} & \multicolumn{3}{|c|}{$\sigma_{x}^{2}$} & \multicolumn{3}{|c|}{$\sigma_{x_{i l}}^{2}$} \\
\hline & & & $\sigma_{s}^{2}$ & $\begin{array}{r}x_{j s} \\
\sigma^{2}\end{array}$ & $\sigma_{l}^{2}$ & $\sigma_{s}^{2}$ & $\sigma_{j}^{2}$ & $\sigma_{l}^{2}$ & $\sigma_{s}^{2}$ & $\sigma_{j l}^{x_{j}} \sigma^{2}$ & $\sigma_{l}^{2}$ \\
\hline \multirow[t]{6}{*}{0,01} & $\mathrm{~W}$ & 3 & 0,0114 & 0,0112 & 0,0110 & 0,0112 & 0,0113 & 0,0113 & 0,0111 & 0,0117 & 0,0118 \\
\hline & W & 5 & 0,0108 & 0,0106 & 0,0109 & 0,0104 & 0,0108 & 0,0105 & 0,0106 & 0,0108 & 0,0104 \\
\hline & W & 20 & 0,010 & 0,010 & 0,0100 & 0,0100 & 0,0100 & 0,0101 & 0,0100 & 0,0100 & 0,010 \\
\hline & RV & 3 & 0,0114 & 0,011 & 0,0112 & 0,0116 & 0,0115 & 0,0118 & 0,0112 & 0,0112 & 0,0117 \\
\hline & RV & 5 & 0,0104 & 0,0109 & 0,0107 & 0,0106 & 0,0102 & 0,0108 & 0,0103 & 0,0101 & 0,0108 \\
\hline & RV & 20 & 0,0100 & 0,0101 & 0,0101 & 0,0102 & 0,010 & 0,0101 & 0,010 & 0,010 & 0,0100 \\
\hline \multirow[t]{6}{*}{0,05} & W & 3 & 0,0517 & 0,0512 & 0,0510 & 0,0513 & 0,0511 & 0,0518 & 0,0510 & 0,0509 & 0,0512 \\
\hline & W & 5 & 0,0507 & 0,0508 & 0,0507 & 0,0508 & 0,0509 & 0,0508 & 0,0507 & 0,0506 & 0,0509 \\
\hline & W & 20 & 0,0500 & 0,0500 & 0,0501 & 0,0501 & 0,0500 & 0,0501 & 0,0500 & 0,0501 & 0,050 \\
\hline & RV & 3 & 0,0518 & 0,0519 & 0,0519 & 0,0514 & 0,0516 & 0,0511 & 0,0519 & 0,0518 & 0,0519 \\
\hline & RV & 5 & 0,0504 & 0,0502 & 0,0506 & 0,0507 & 0,0509 & 0,0501 & 0,0501 & 0,0501 & 0,0507 \\
\hline & RV & 20 & 0,0500 & 0,0500 & 0,0501 & 0,0500 & 0,0501 & 0,050 & 0,050 & 0,0500 & 0,0501 \\
\hline \multirow[t]{6}{*}{0,10} & W & 3 & 0,118 & 0,1121 & 0,118 & 0,1164 & 0,117 & 0,112 & 0,113 & 0,1156 & 0,1120 \\
\hline & W & 5 & 0,103 & 0,109 & 0,103 & 0,105 & 0,101 & 0,105 & 0,103 & 0,103 & 0,105 \\
\hline & W & 20 & 0,1002 & 0,1001 & 0,1001 & 0,1005 & 0,10 & 0,1003 & 0,10 & 0,1002 & 0,1003 \\
\hline & RV & 3 & 0,119 & 0,116 & 0,116 & 0,11 & 0,115 & 0,117 & 0,116 & 0,114 & 0,117 \\
\hline & RV & 5 & 0,108 & 0,103 & 0,102 & 0,108 & 0,101 & 0,103 & 0,102 & 0,102 & 0,104 \\
\hline & RV & 20 & 0,1007 & 0,1001 & 0,10 & 0,1002 & 0,10 & 0,1007 & 0,10 & 0,1007 & 0,1006 \\
\hline
\end{tabular}


Tabela 36: Tamanhos empíricos dos testes de Wald e Razão de Verossimilhança para a sexta hipótese com $\mathrm{p}=3$ e $\mathrm{m}=5$ - Distribuição t de Student.

\begin{tabular}{|c|c|c|c|c|c|c|c|c|c|c|c|}
\hline \multirow[b]{2}{*}{ nivel } & \multirow[b]{2}{*}{ teste } & \multirow[b]{2}{*}{ ni } & \multicolumn{3}{|c|}{$\sigma_{x_{i s}}^{2}$} & \multicolumn{3}{|c|}{$\sigma_{x}^{2}$} & \multicolumn{3}{|c|}{$\sigma_{x_{i l}}^{2}$} \\
\hline & & & $\sigma_{s}^{2}$ & $\begin{array}{r}x_{j s} \\
\sigma^{2}\end{array}$ & $\sigma_{l}^{2}$ & $\sigma_{s}^{2}$ & $\sigma_{j}^{2}$ & $\sigma_{l}^{2}$ & $\sigma_{s}^{2}$ & $\sigma_{j l}^{x_{j}} \sigma^{2}$ & $\sigma_{l}^{2}$ \\
\hline \multirow[t]{6}{*}{0,01} & $\mathrm{~W}$ & 3 & 0,0114 & 0,0107 & 0,0113 & 0,0118 & 0,0112 & 0,0113 & 0,0105 & 0,011 & 0,0113 \\
\hline & W & 5 & 0,0108 & 0,0104 & 0,0106 & 0,0102 & 0,0102 & 0,0106 & 0,0103 & 0,0109 & 0,0104 \\
\hline & W & 20 & 0,0100 & 0,01 & 0,0100 & 0,01 & 0,0100 & 0,0100 & 0,01 & 0,0101 & 0,0100 \\
\hline & RV & 3 & 0,0112 & 0,0108 & 0,0114 & 0,011 & 0,0112 & 0,0112 & 0,0109 & 0,011 & 0,011 \\
\hline & RV & 5 & 0,0103 & 0,0106 & 0,0108 & 0,0109 & 0,0108 & 0,0106 & 0,0107 & 0,0108 & 0,0107 \\
\hline & RV & 20 & 0,0100 & 0,0100 & 0,0100 & 0,0101 & 0,010 & 0,0100 & 0,01 & 0,0100 & 0,0101 \\
\hline \multirow[t]{6}{*}{0,05} & W & 3 & 0,0509 & 0,0511 & 0,0504 & 0,051 & 0,0514 & 0,0503 & 0,0512 & 0,0518 & 0,0511 \\
\hline & W & 5 & 0,0502 & 0,0509 & 0,0500 & 0,0508 & 0,0502 & 0,0501 & 0,0509 & 0,0511 & 0,0509 \\
\hline & W & 20 & 0,0501 & 0,0500 & 0,050 & 0,0500 & 0,050 & 0,050 & 0,0500 & 0,0503 & 0,0501 \\
\hline & RV & 3 & 0,0507 & 0,0511 & 0,0503 & 0,051 & 0,0514 & 0,0513 & 0,0516 & 0,0512 & 0,0511 \\
\hline & RV & 5 & 0,0501 & 0,0504 & 0,0501 & 0,0508 & 0,0507 & 0,0509 & 0,051 & 0,0511 & 0,0506 \\
\hline & RV & 20 & 0,0501 & 0,050 & 0,050 & 0,0500 & 0,0500 & 0,0500 & 0,0502 & 0,0507 & 0,0500 \\
\hline \multirow[t]{6}{*}{0,10} & W & 3 & 0,1031 & 0,1044 & 0,1038 & 0,1038 & 0,1028 & 0,1038 & 0,1045 & 0,1032 & 0,1036 \\
\hline & W & 5 & 0,1011 & 0,1032 & 0,1016 & 0,1028 & 0,101 & 0,1026 & 0,1031 & 0,1022 & 0,1021 \\
\hline & W & 20 & 0,10 & 0,101 & 0,10 & 0,1008 & 0,10 & 0,1009 & 0,101 & 0,1004 & 0,1002 \\
\hline & RV & 3 & 0,1063 & 0,1079 & 0,1072 & 0,1071 & 0,1073 & 0,1073 & 0,108 & 0,1066 & 0,1079 \\
\hline & RV & 5 & 0,101 & 0,1046 & 0,1035 & 0,1022 & 0,102 & 0,1011 & 0,106 & 0,1021 & 0,101 \\
\hline & RV & 20 & 0,10 & 0,1003 & 0,1001 & 0,10 & 0,10 & 0,1007 & 0,1006 & 0,1001 & 0,10 \\
\hline
\end{tabular}




\section{Apêndice $\mathrm{C}$}

Neste Apêndice apresentaremos os resultados da simulação do poder dos testes de Wald e RV para a distribuição Normal e t de Student, com 3 e 20 réplicas para 6 laboratórios e 10 rotações e depois com 5 réplicas para 3 laboratórios e 5 rotações. Apresentaremos os gráficos de maneira reduzida, dispostos da seguinte maneira: poder do teste de Wald para a hipótese 1, poder para o teste RV para a hipótese 1. Ao lado, analogamente para as hipóteses 2 e 3 . Logo abaixo, poder do teste de Wald para a hipótese 4, poder para o teste RV para a hipótese 4. Ao lado, analogamente para as hipóteses 5 e 6 . Tais gráficos seguirão a mesma ordem vista na seção 5.2, isto é, 6 primeiras hipóteses com $\sigma_{x j s}^{2}$ e $\sigma_{s}^{2}$, seguida de mais seis hipóteses com $\sigma_{x j s}^{2}$ e $\sigma^{2}$ e mais seis com $\sigma_{x j s}^{2}$ e $\sigma_{l}^{2}$. Analogamente para o restante com $\sigma_{x j}^{2}$ e $\sigma_{s}^{2}, \sigma^{2}, \sigma_{l}^{2}$ e com $\sigma_{x j l}^{2}$ e $\sigma_{s}^{2}, \sigma^{2}, \sigma_{l}^{2}$ 

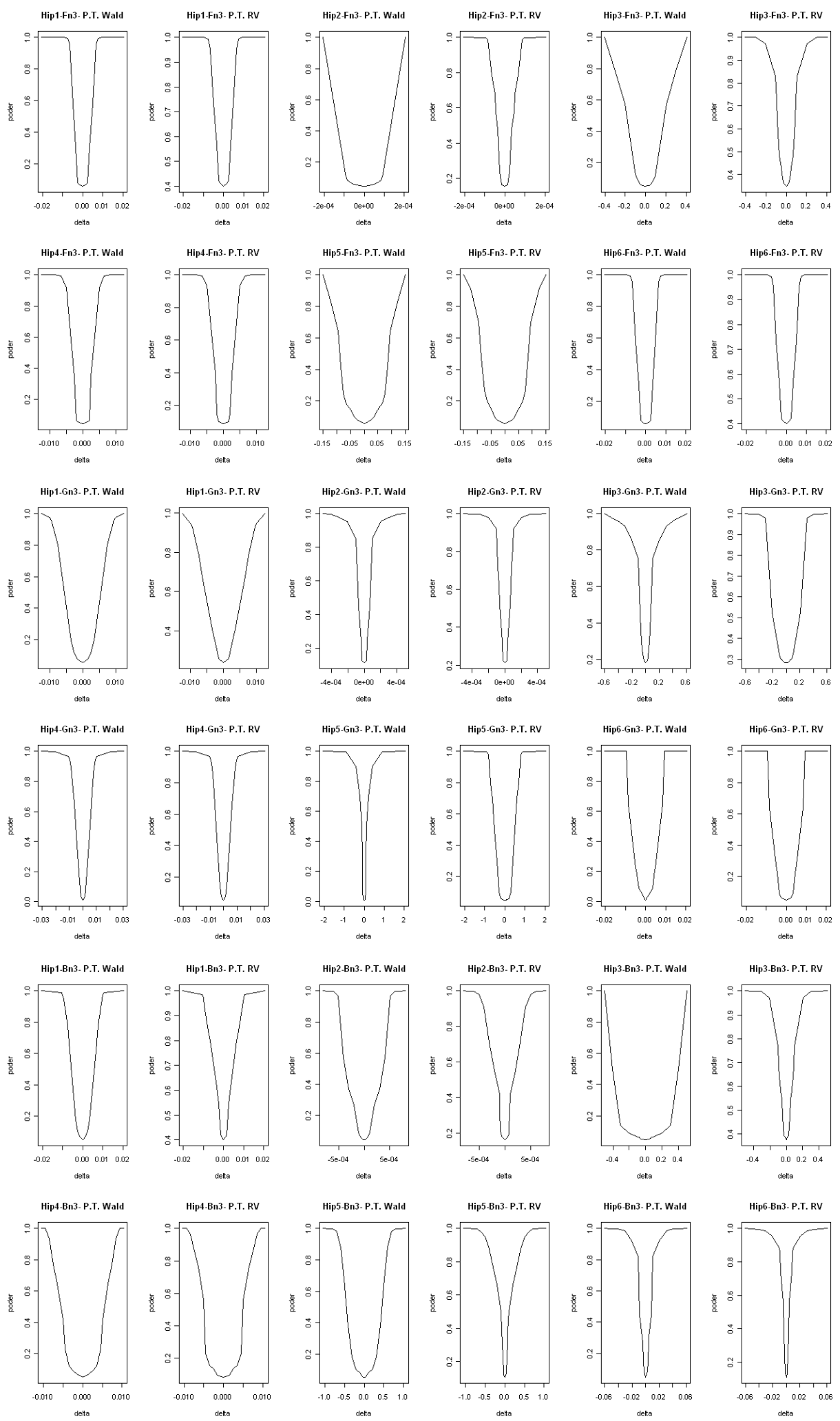

Figura 19: Gráficos do P. T. de Wald e RV para 3 réplicas- distribuição Normal- 6 laboratórios e 10 rotações. 

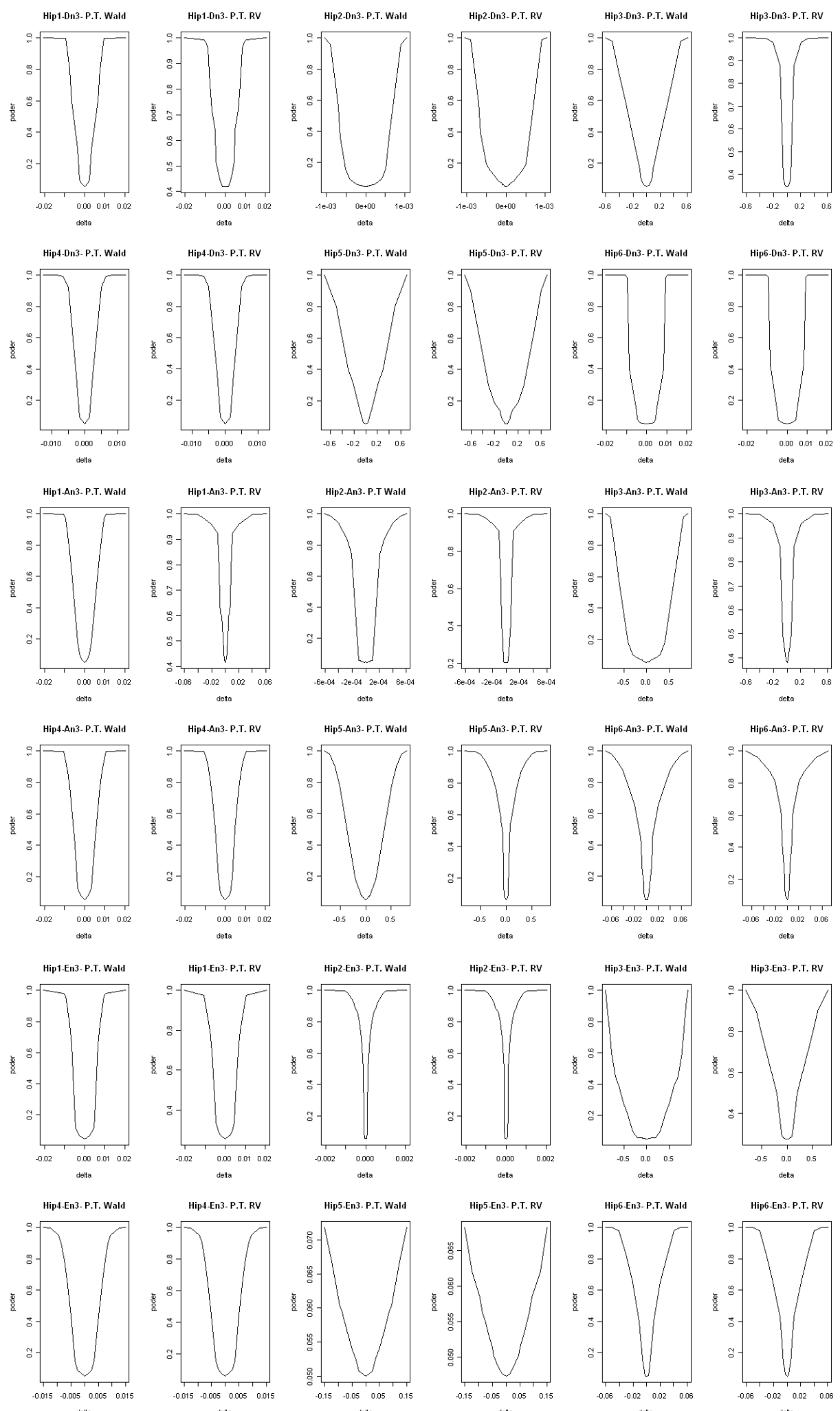

Figura 20: Gráficos do P. T. de Wald e RV para 3 réplicas- distribuição Normal- 6 laboratórios e 10 rotações. 

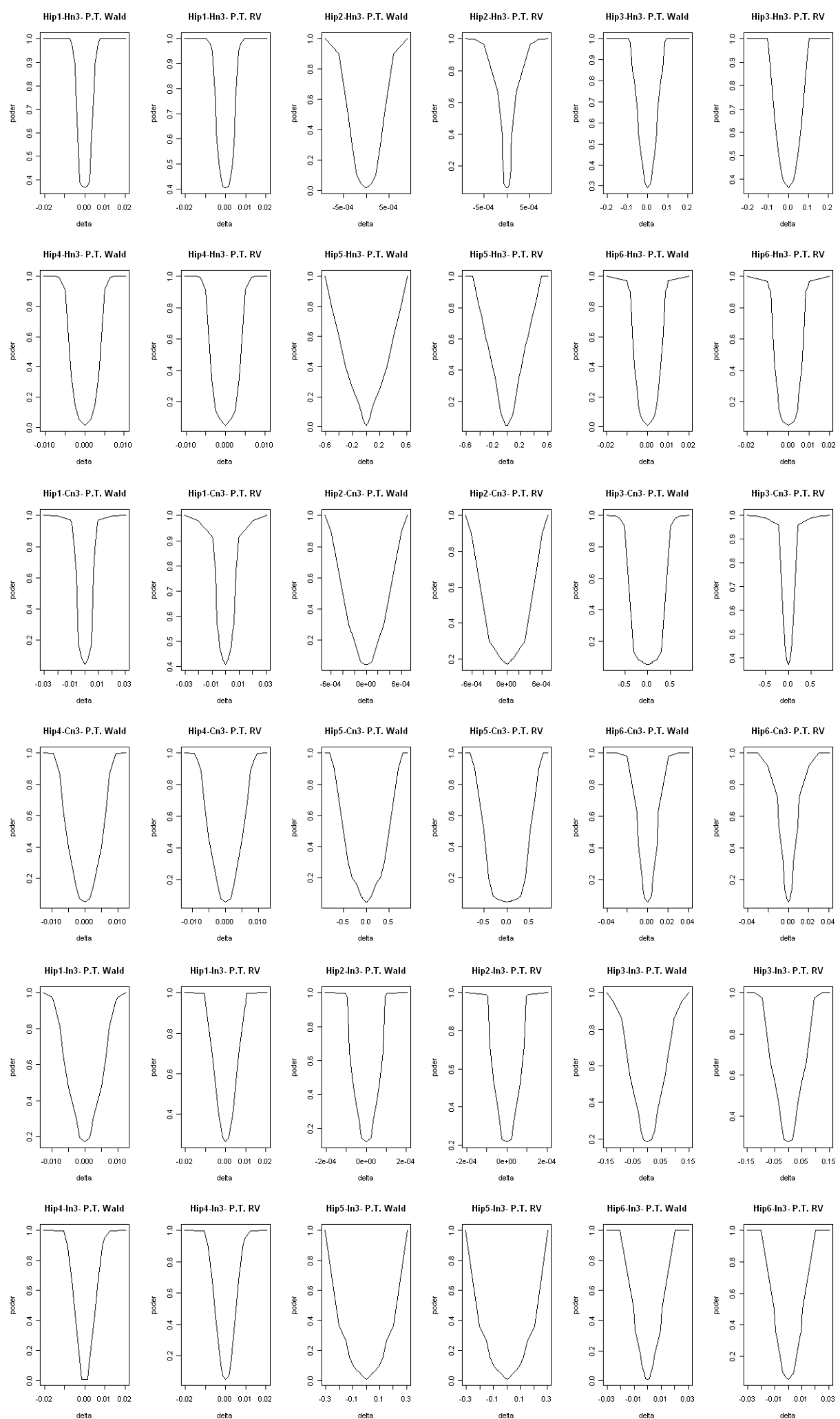

Figura 21: Gráficos do P. T. de Wald e RV para 3 réplicas- distribuição Normal- 6 laboratórios e 10 rotações. 

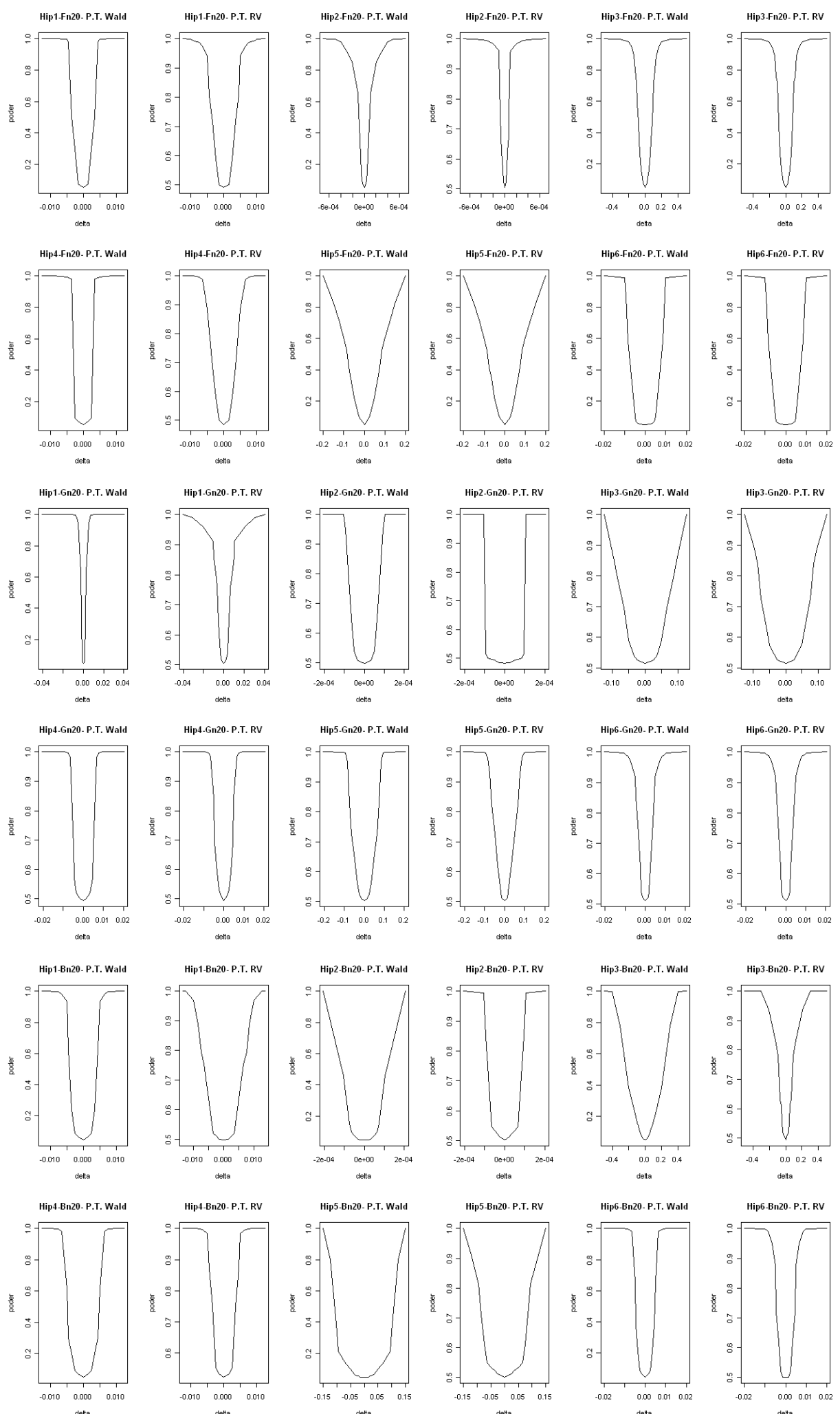

Figura 22: Gráficos do P. T. de Wald e RV para 20 réplicas- distribuição Normal- 6 laboratórios e 10 rotações. 

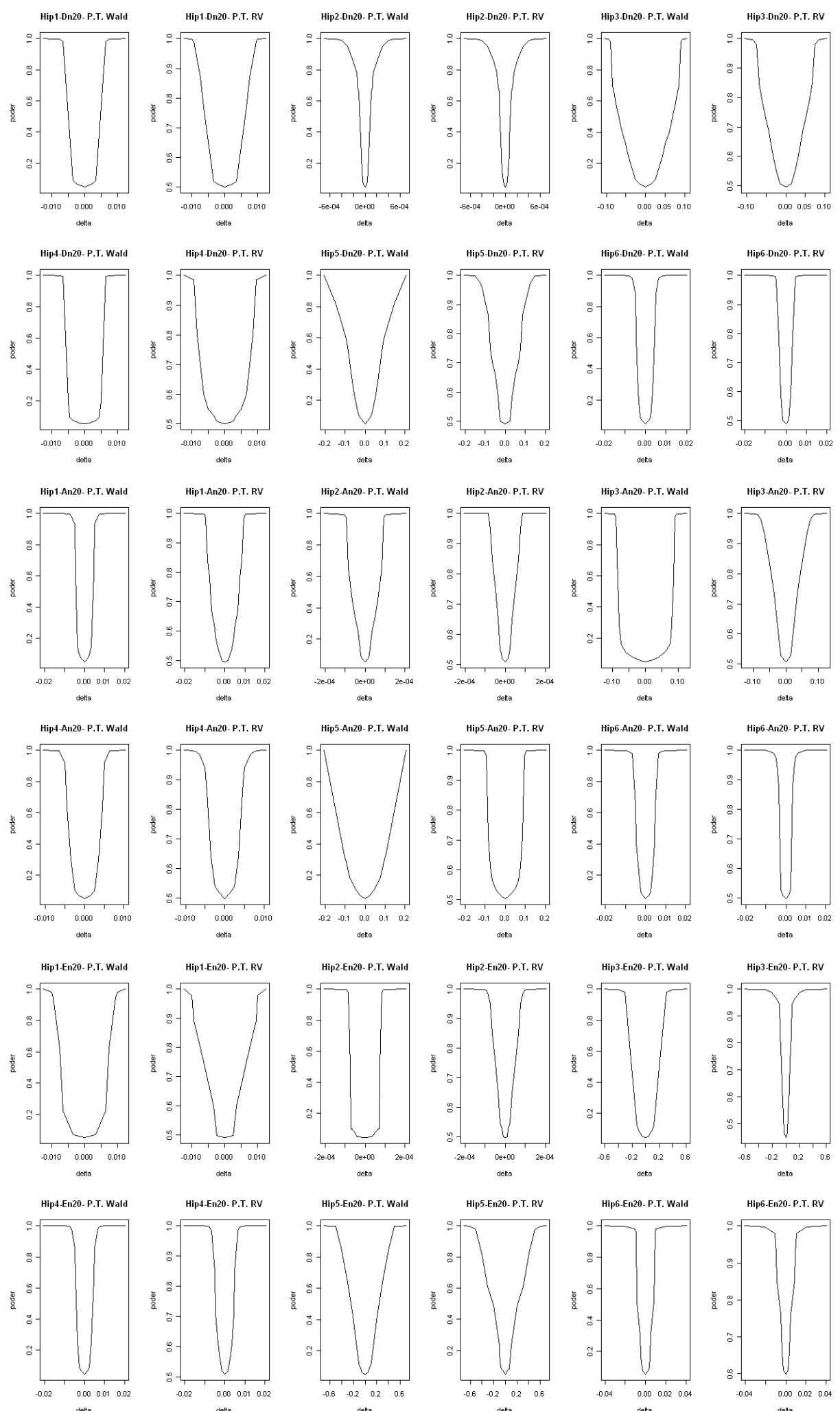

Figura 23: Gráficos do P. T. de Wald e RV para 20 réplicas- distribuição Normal- 6 laboratórios e 10 rotações. 

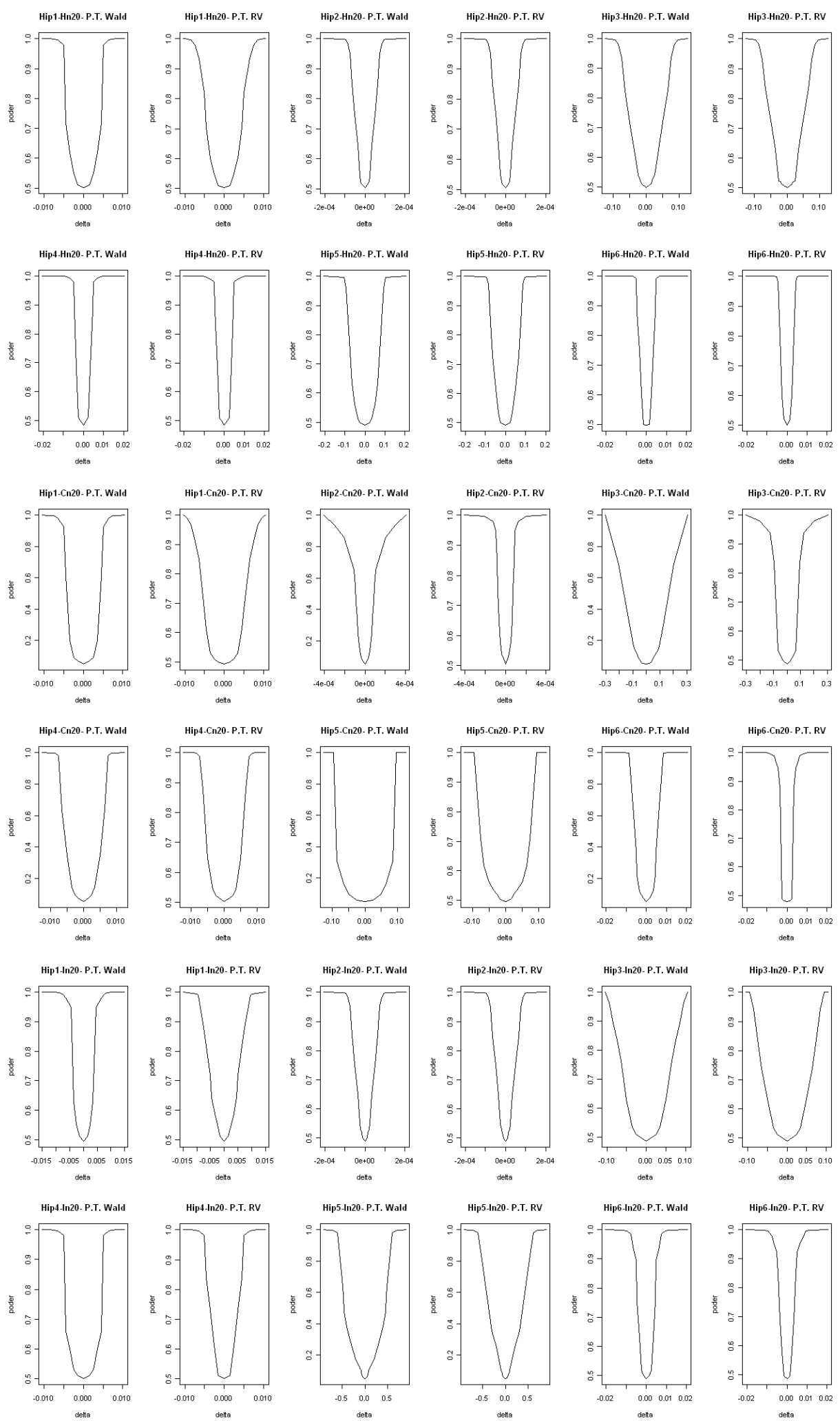

Figura 24: Gráficos do P. T. de Wald e RV para 20 réplicas- distribuição Normal- 6 laboratórios e 10 rotações. 

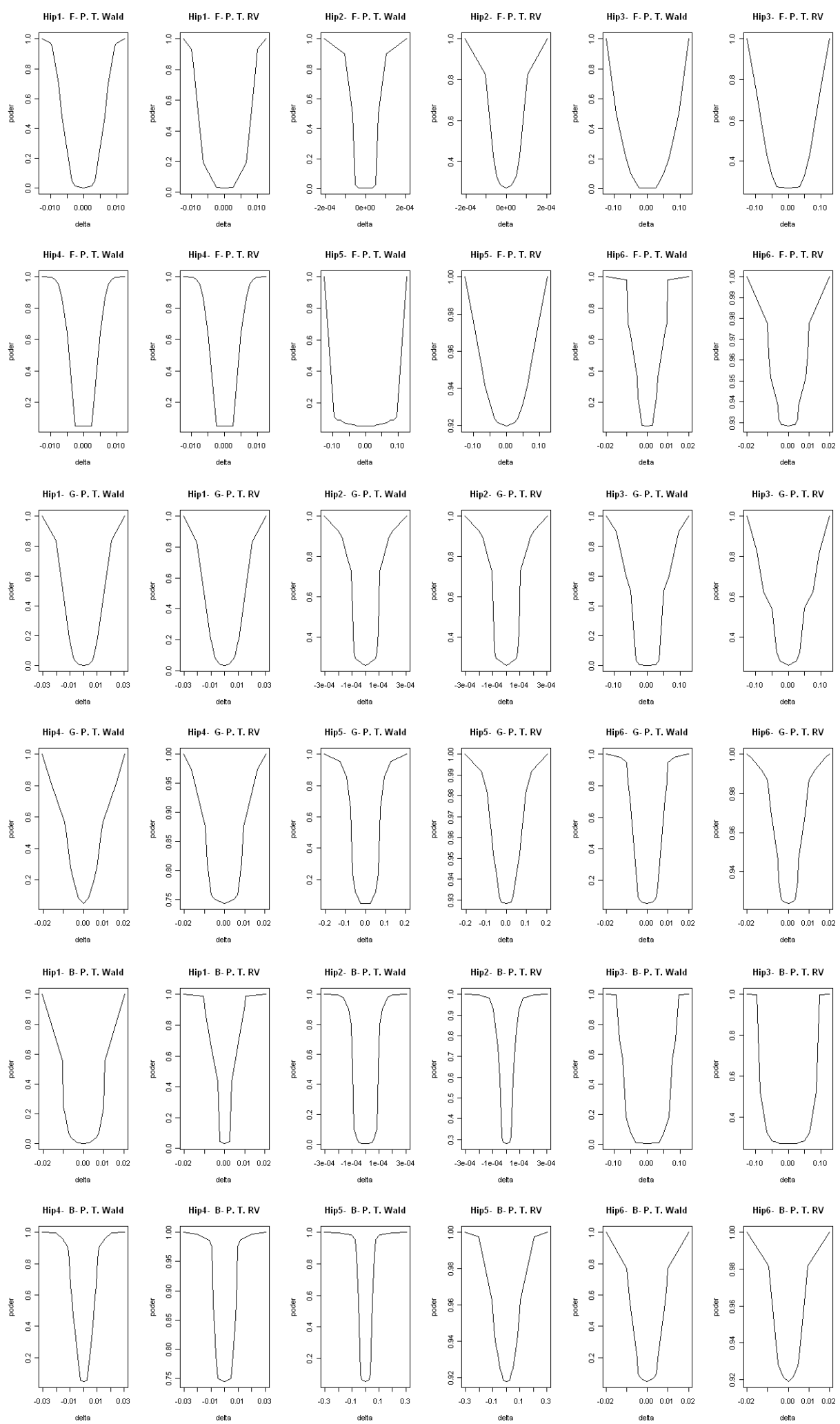

Figura 25: Gráficos do P. T. de Wald e RV para 3 réplicas- distribuição Normal- 3 laboratórios e 5 rotações. 

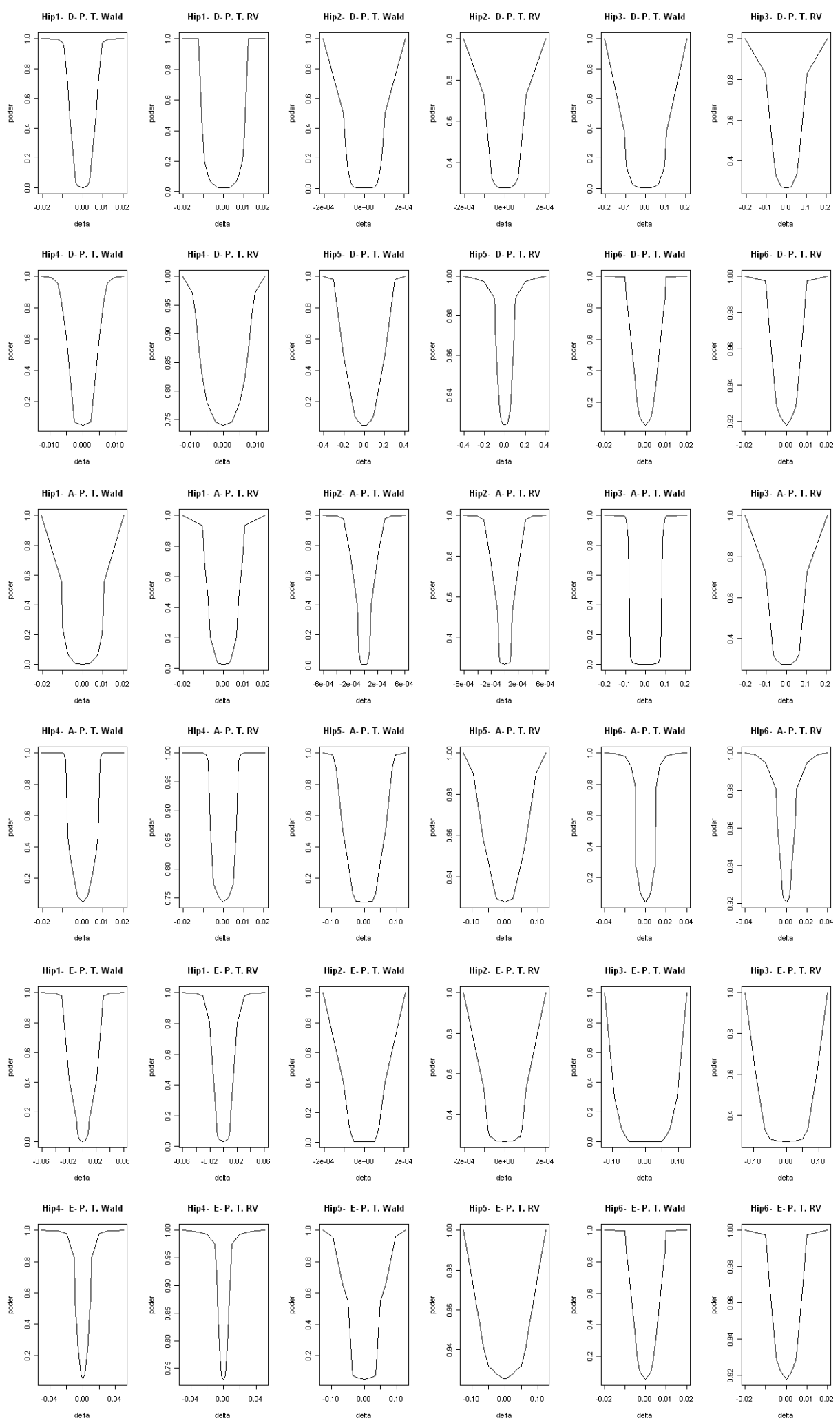

Figura 26: Gráficos do P. T. de Wald e RV para 3 réplicas- distribuição Normal- 3 laboratórios e 5 rotações. 

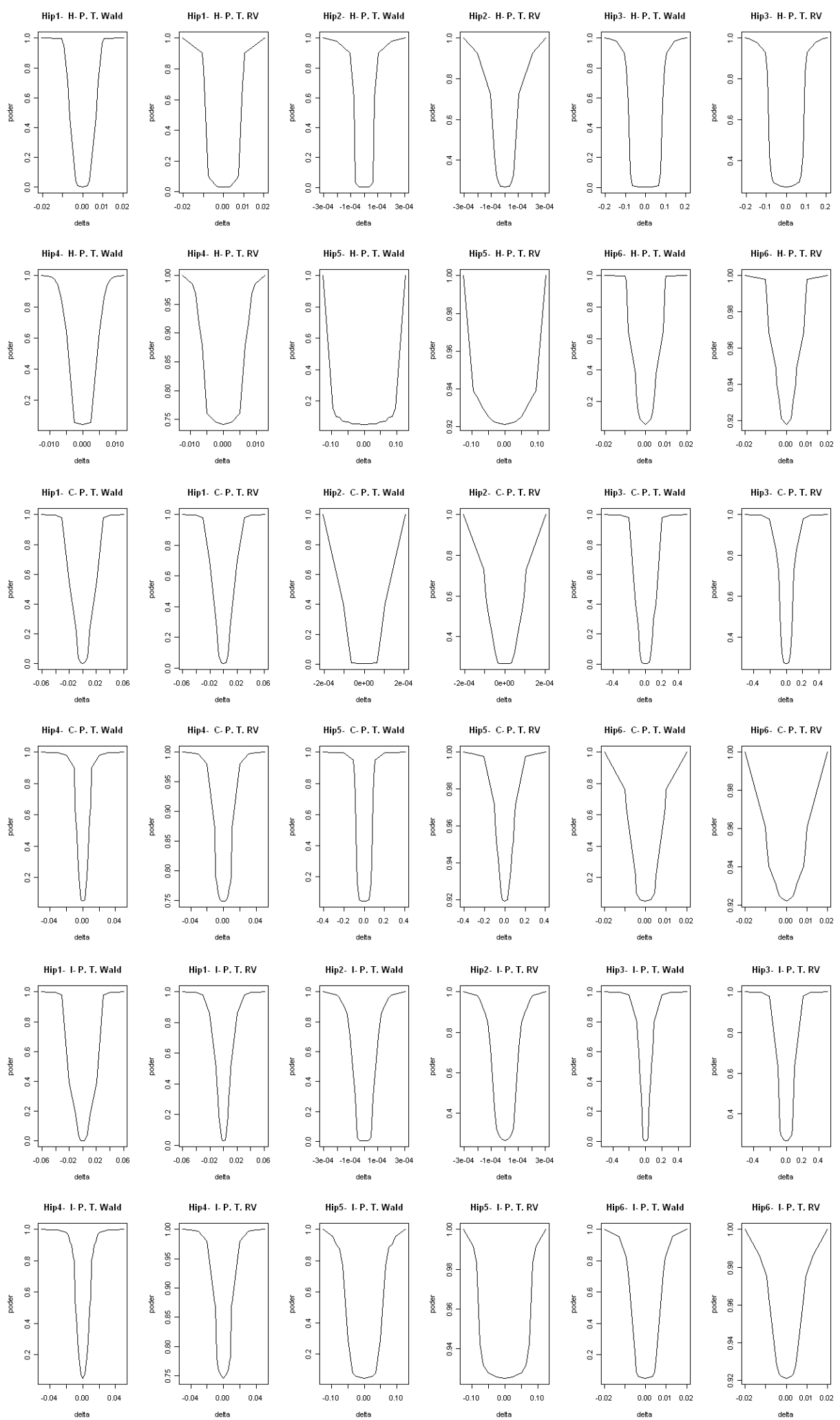

Figura 27: Gráficos do P. T. de Wald e RV para 3 réplicas- distribuição Normal- 3 laboratórios e 5 rotações. 

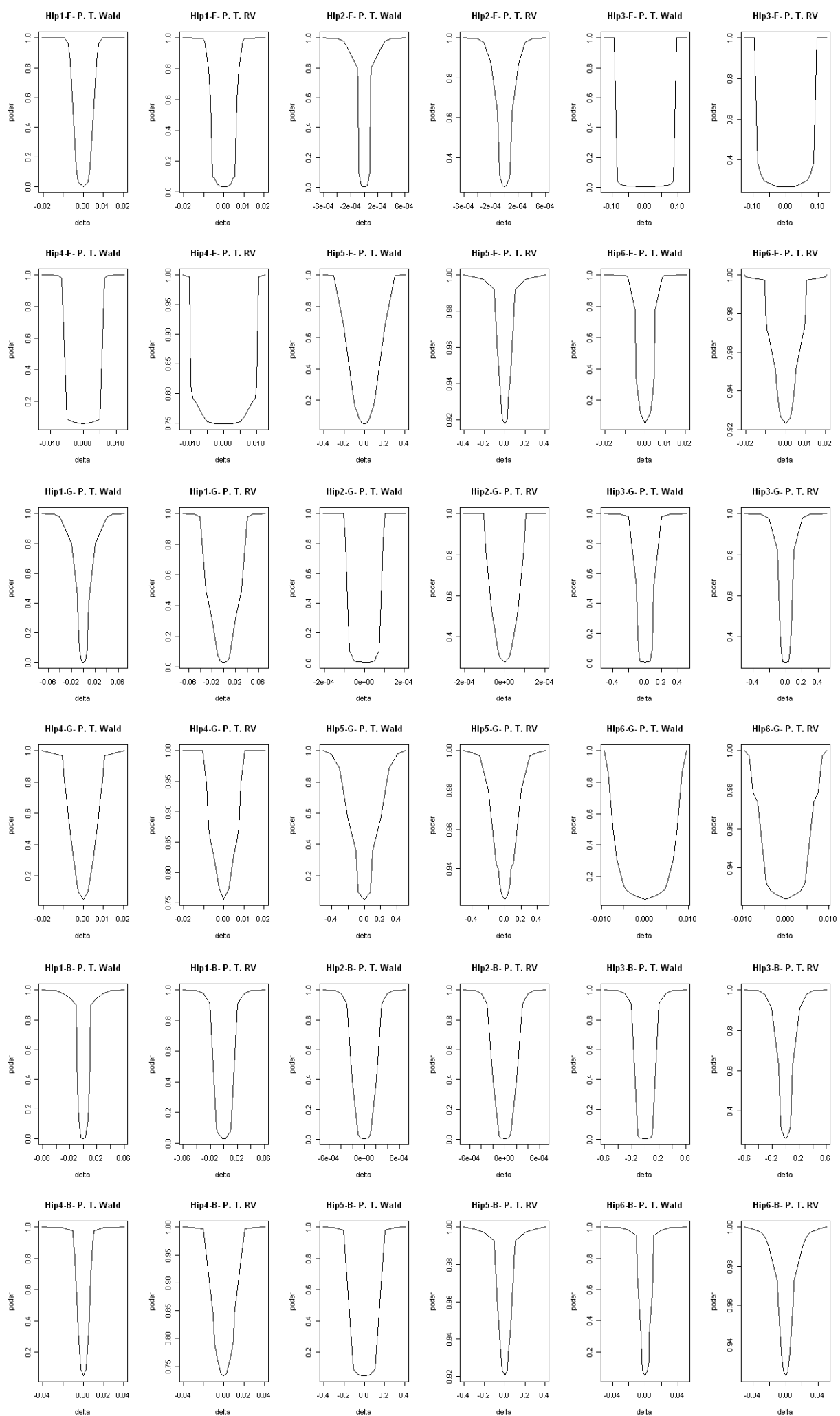

Figura 28: Gráficos do P. T. de Wald e RV para 5 réplicas- distribuição Normal- 3 laboratórios e 5 rotações. 

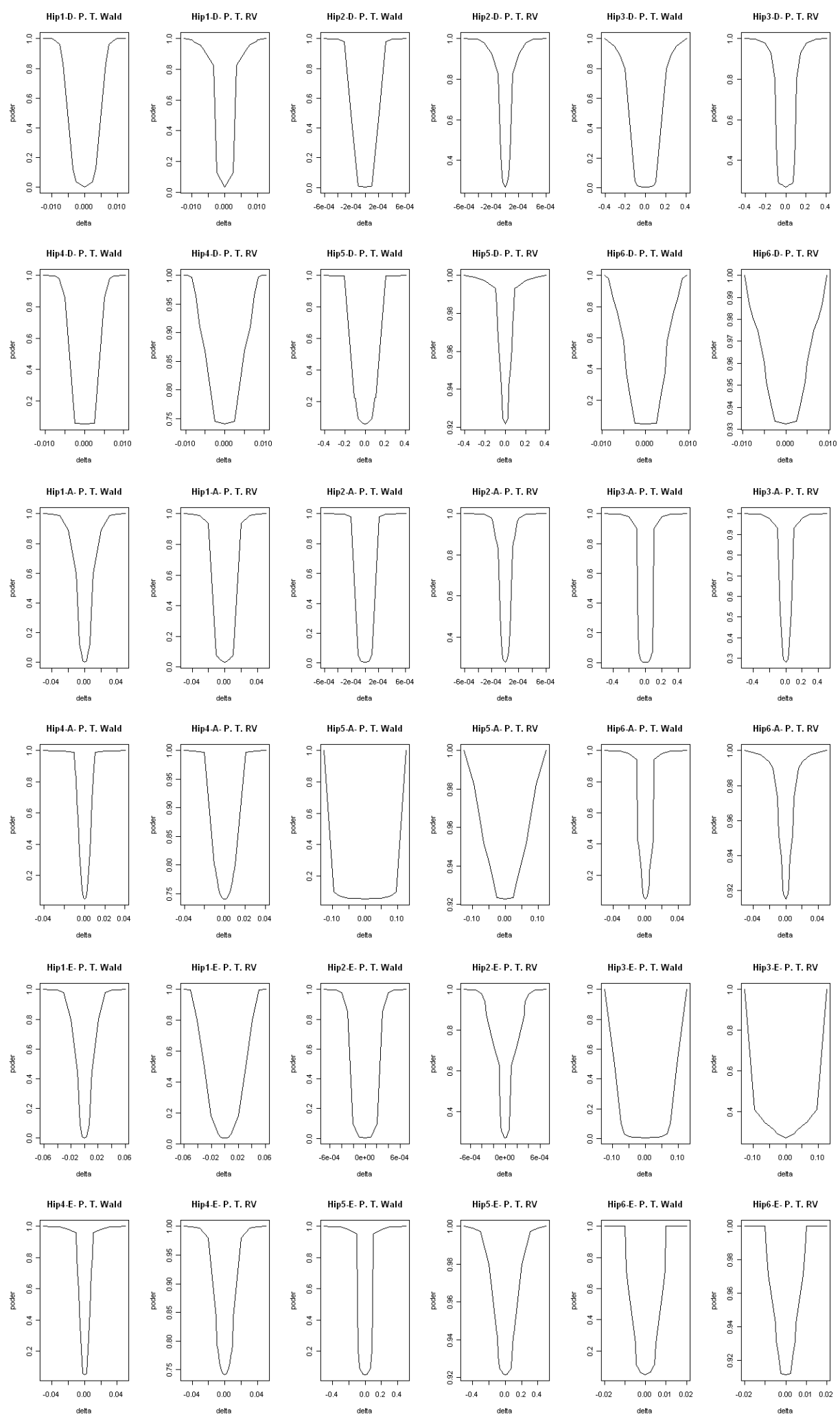

Figura 29: Gráficos do P. T. de Wald e RV para 5 réplicas- distribuição Normal- 3 laboratórios e 5 rotações. 

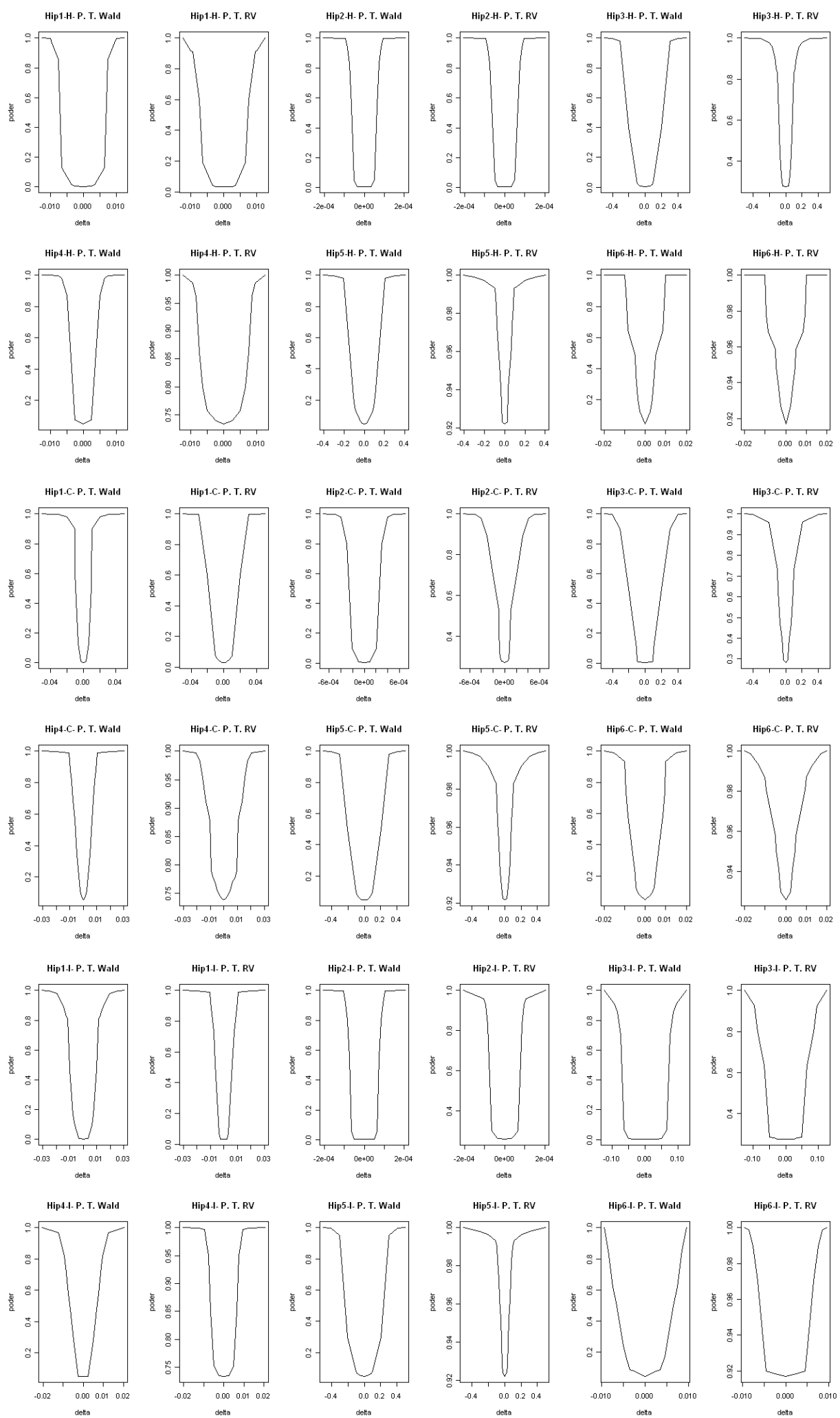

Figura 30: Gráficos do P. T. de Wald e RV para 5 réplicas- distribuição Normal- 3 laboratórios e 5 rotações. 

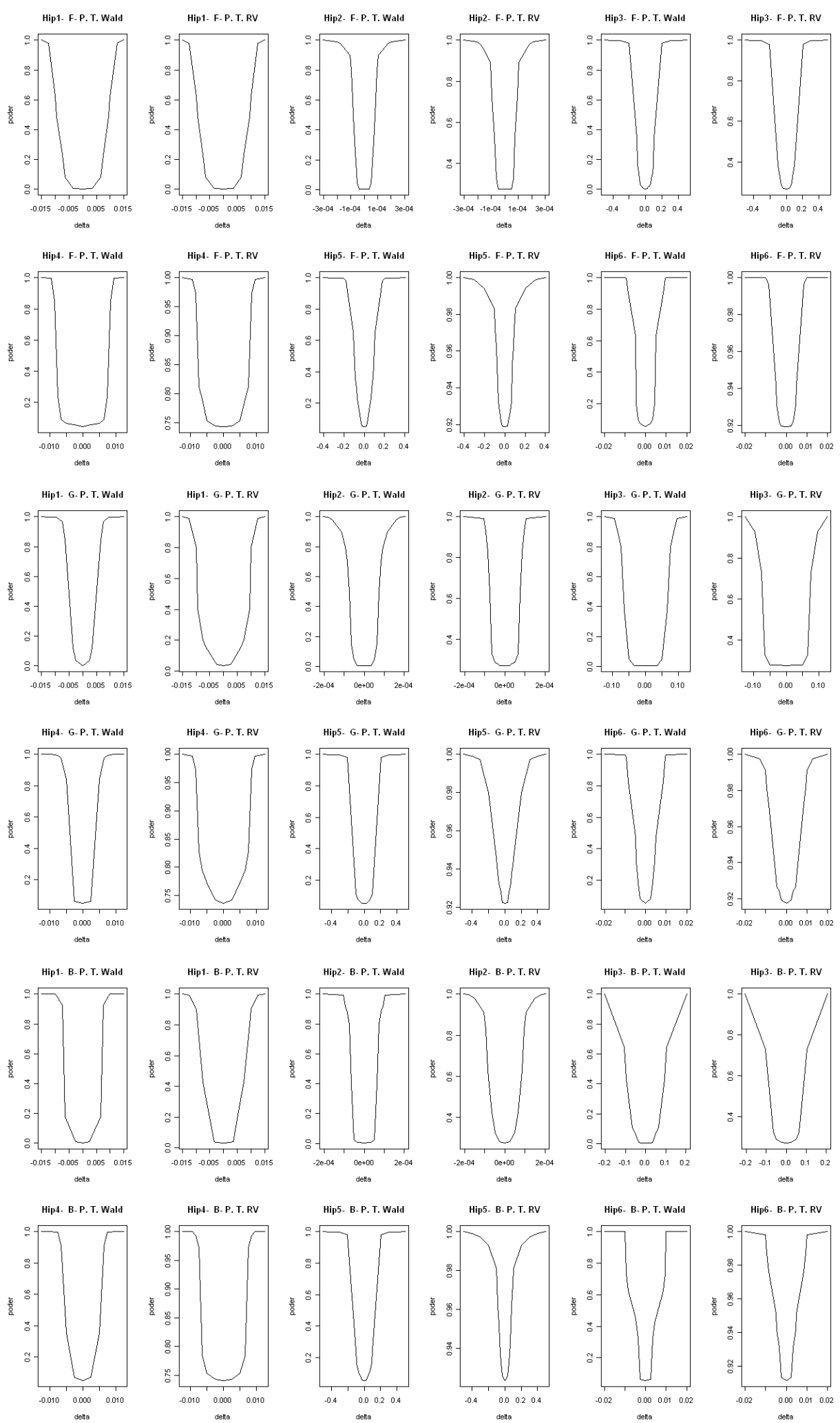

Figura 31: Gráficos do P. T. de Wald e RV para 20 réplicas- distribuição Normal- 3 laboratórios e 5 rotações. 

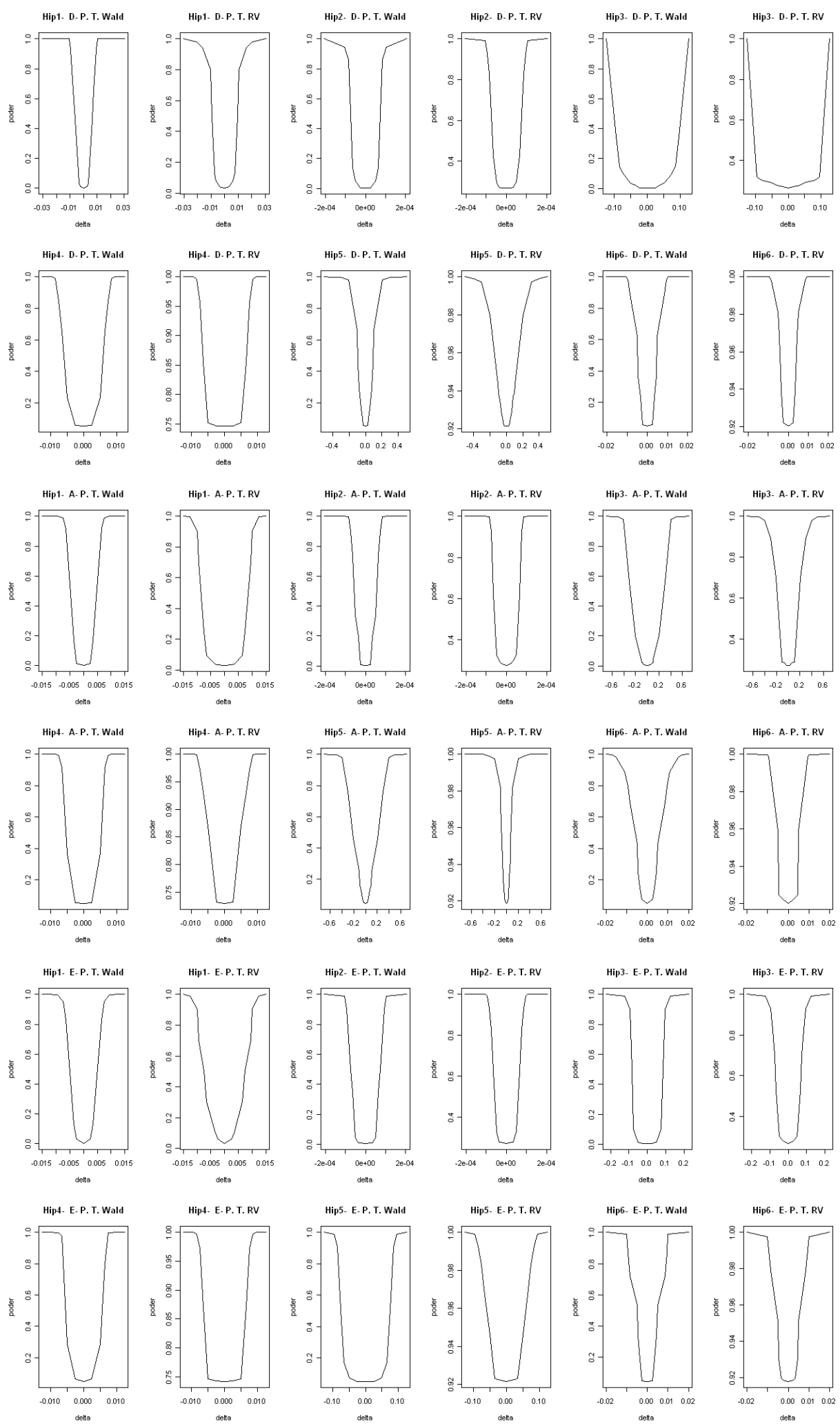

Figura 32: Gráficos do P. T. de Wald e RV para 20 réplicas- distribuição Normal- 3 laboratórios e 5 rotações. 

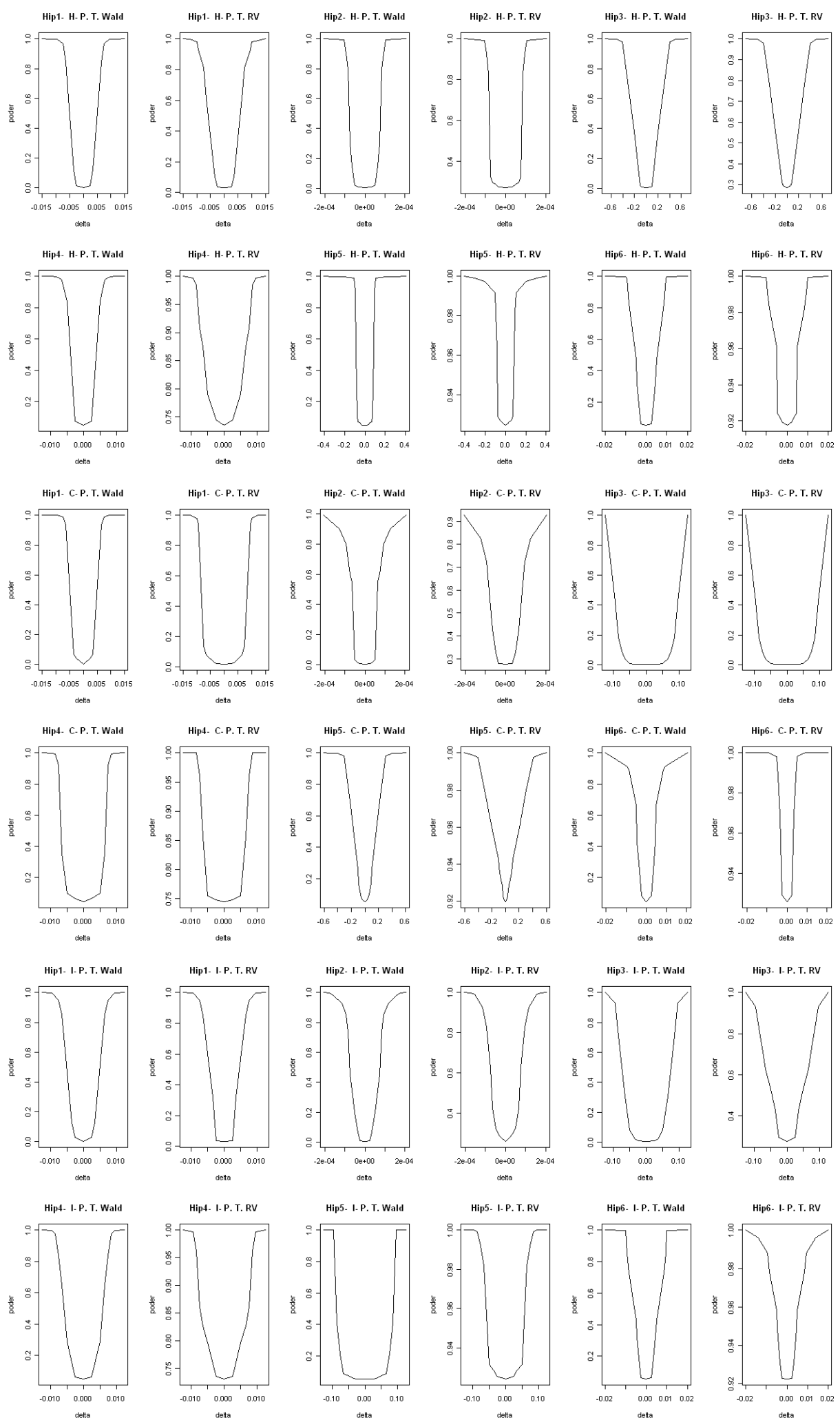

Figura 33: Gráficos do P. T. de Wald e RV para 20 réplicas- distribuição Normal- 3 laboratórios e 5 rotações. 

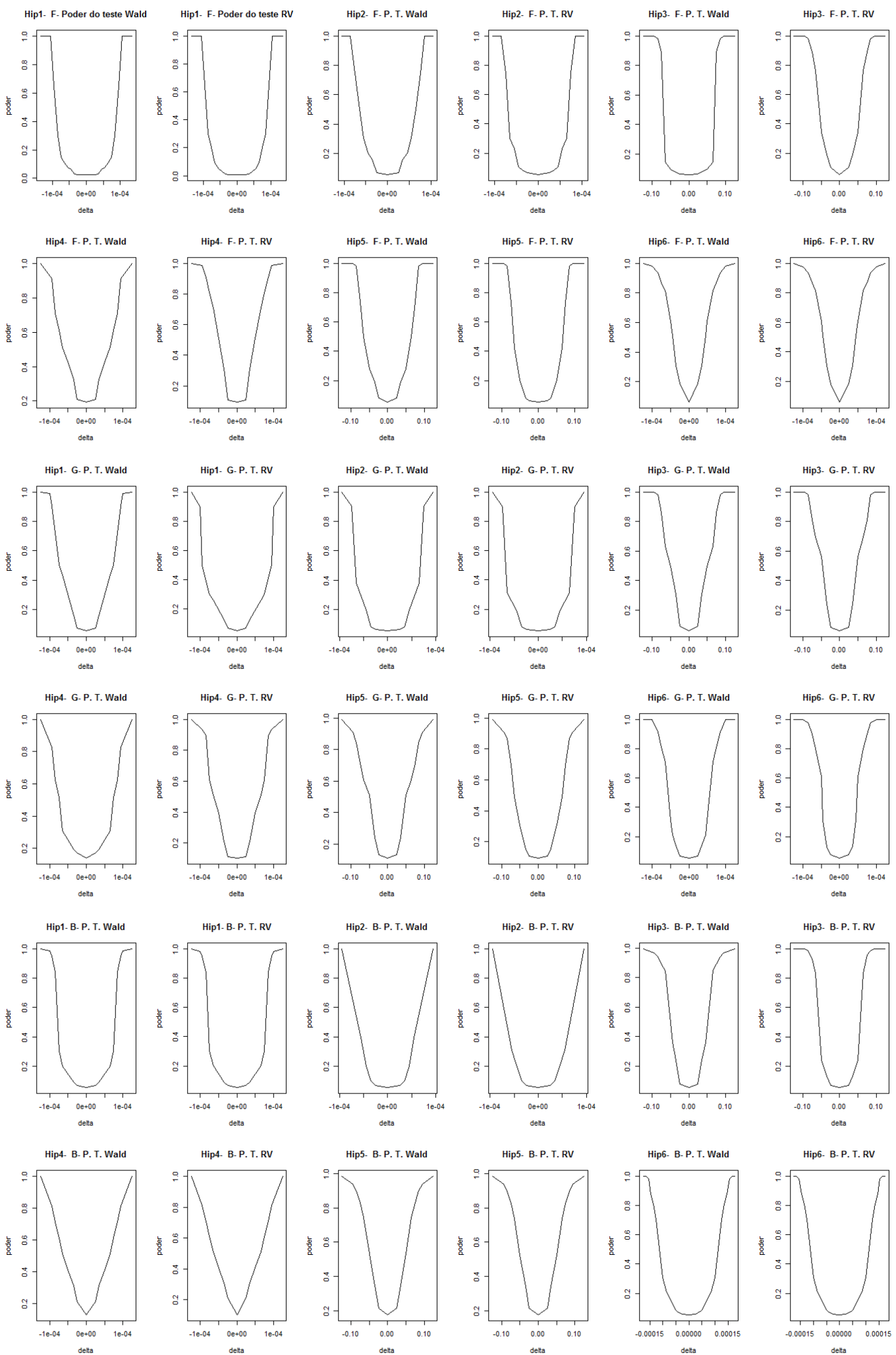

Figura 34: Gráficos do P. T. de Wald e RV para 3 réplicas- distribuição t de Student6 laboratórios e 10 rotações. 

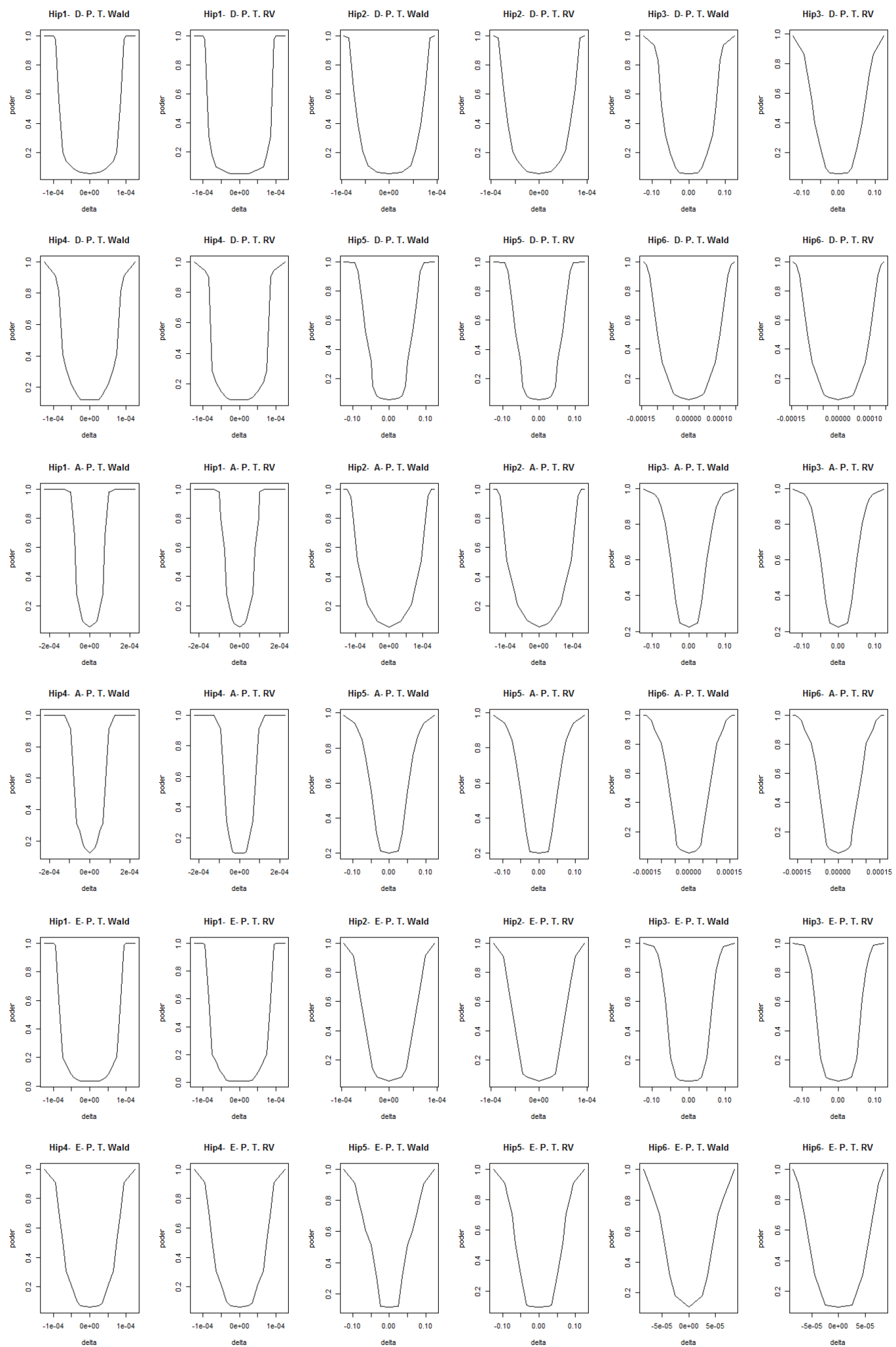

Figura 35: Gráficos do P. T. de Wald e RV para 3 réplicas- distribuição t de Student6 laboratórios e 10 rotações. 

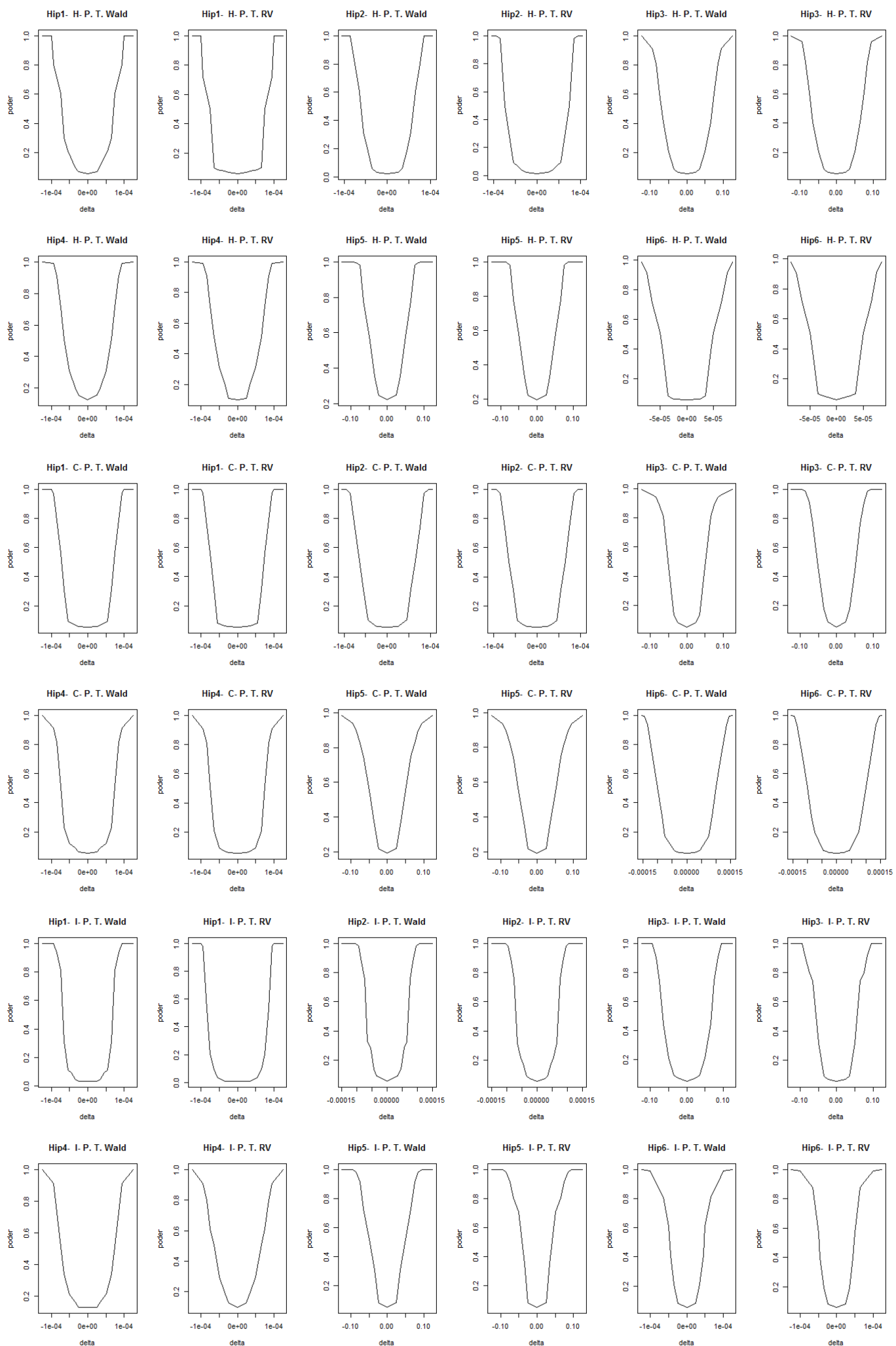

Figura 36: Gráficos do P. T. de Wald e RV para 3 réplicas- distribuição t de Student6 laboratórios e 10 rotações. 

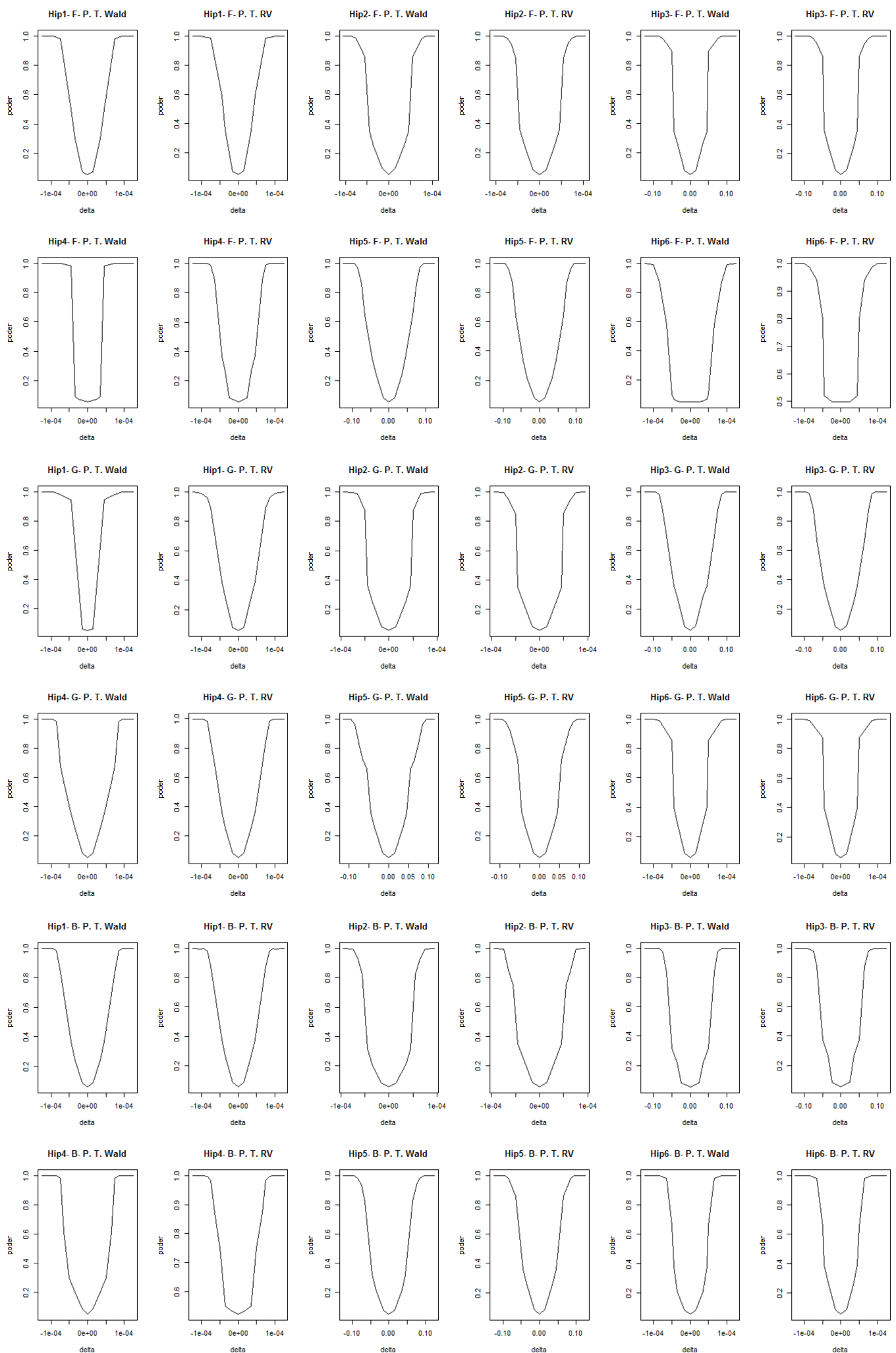

Figura 37: Gráficos do P. T. de Wald e RV para 20 réplicas- distribuição t de Student6 laboratórios e 10 rotações. 

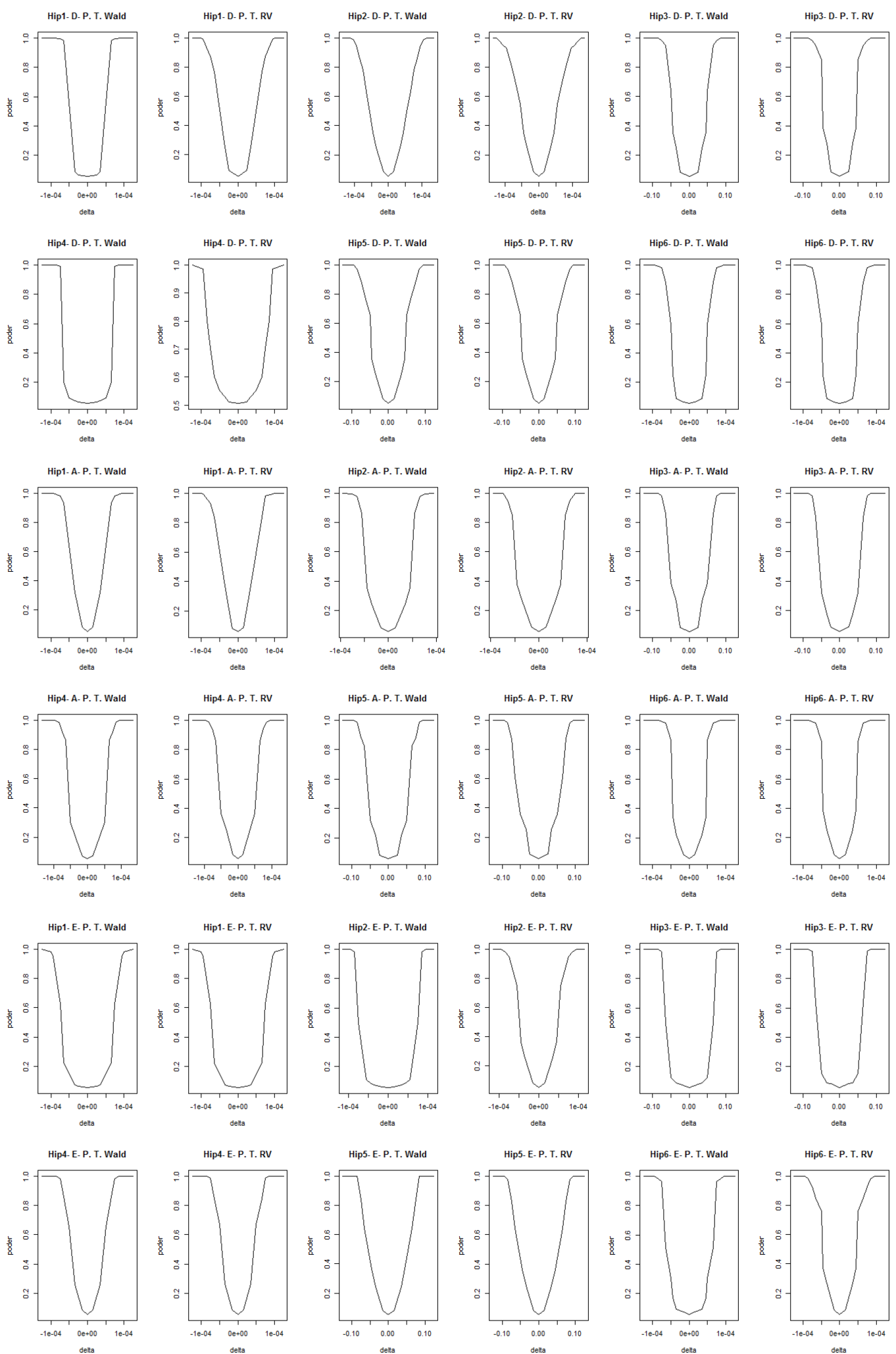

Figura 38: Gráficos do P. T. de Wald e RV para 20 réplicas- distribuição t de Student6 laboratórios e 10 rotações. 

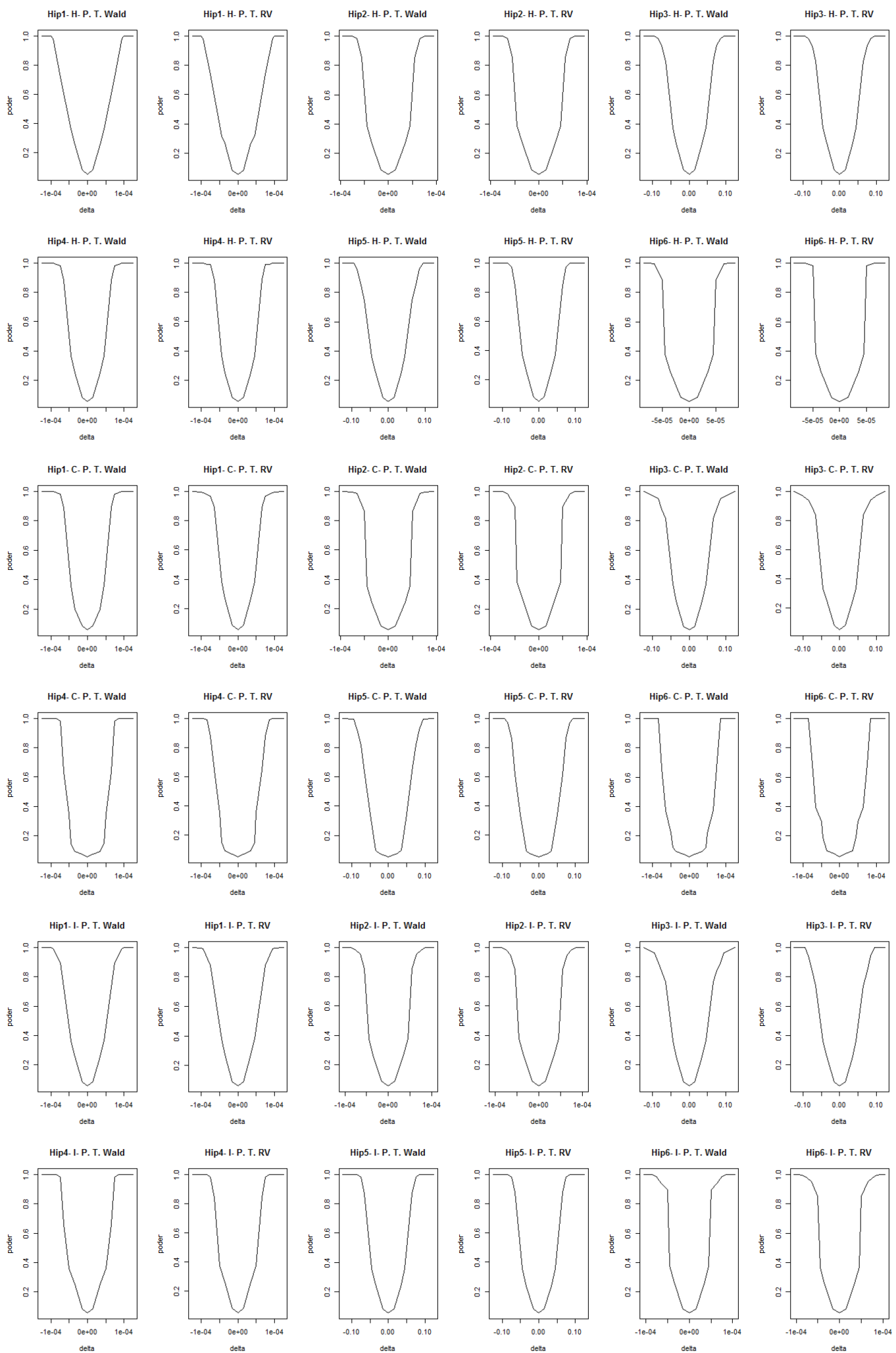

Figura 39: Gráficos do P. T. de Wald e RV para 20 réplicas- distribuição t de Student6 laboratórios e 10 rotações. 

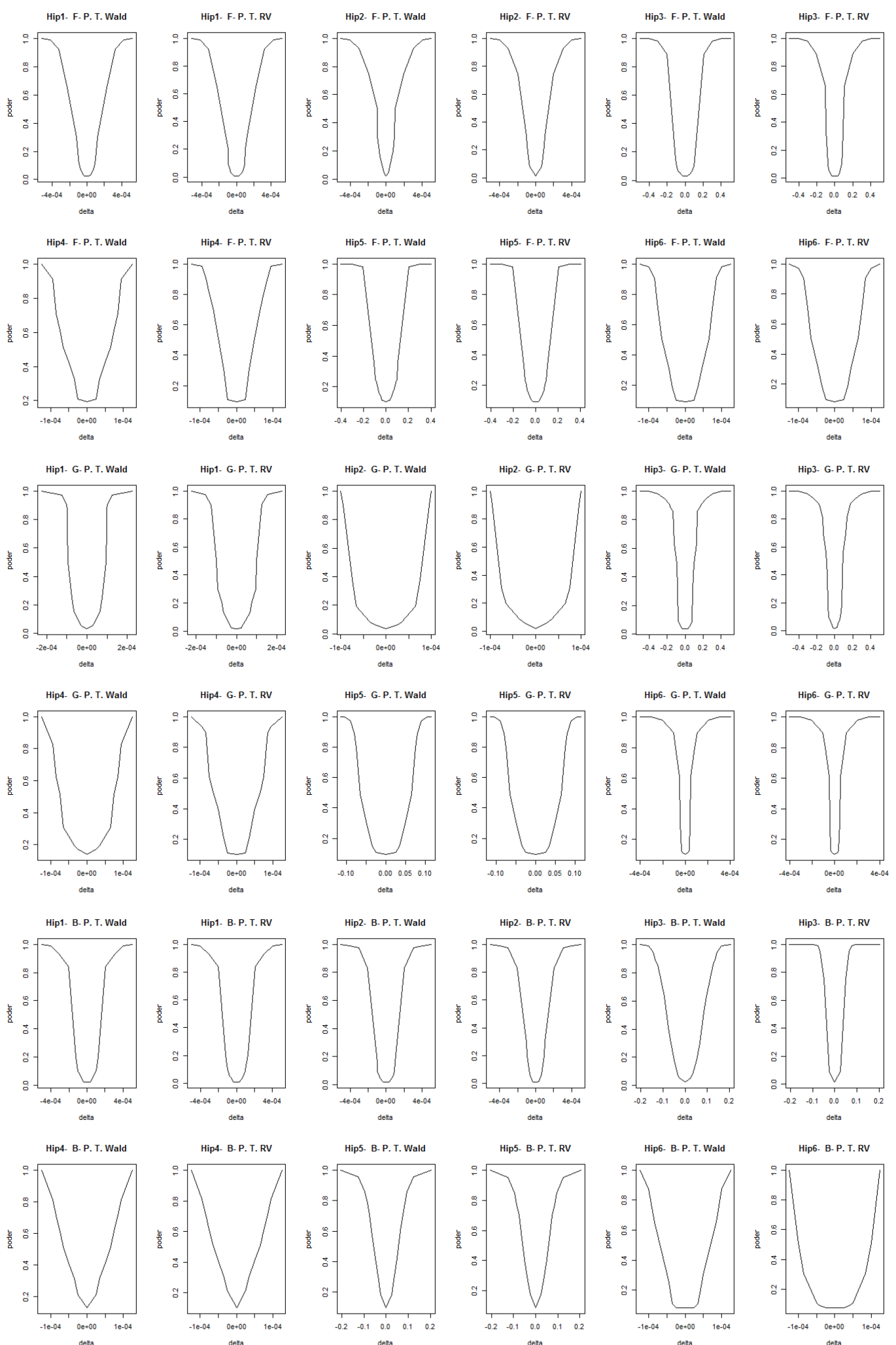

Figura 40: Gráficos do P. T. de Wald e RV para 3 réplicas- distribuição t de Student3 laboratórios e 5 rotações. 

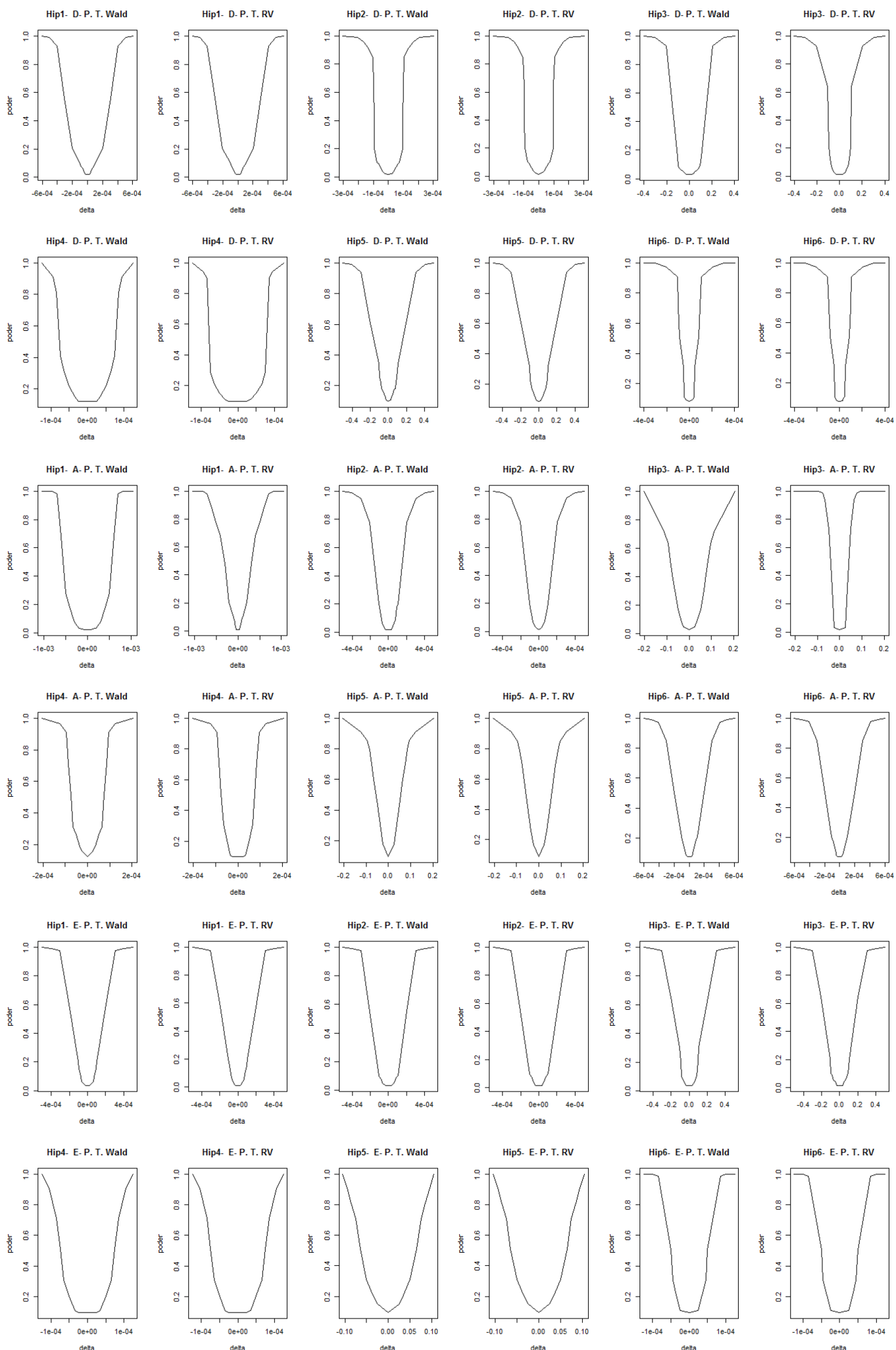

Figura 41: Gráficos do P. T. de Wald e RV para 3 réplicas- distribuição t de Student3 laboratórios e 5 rotações. 

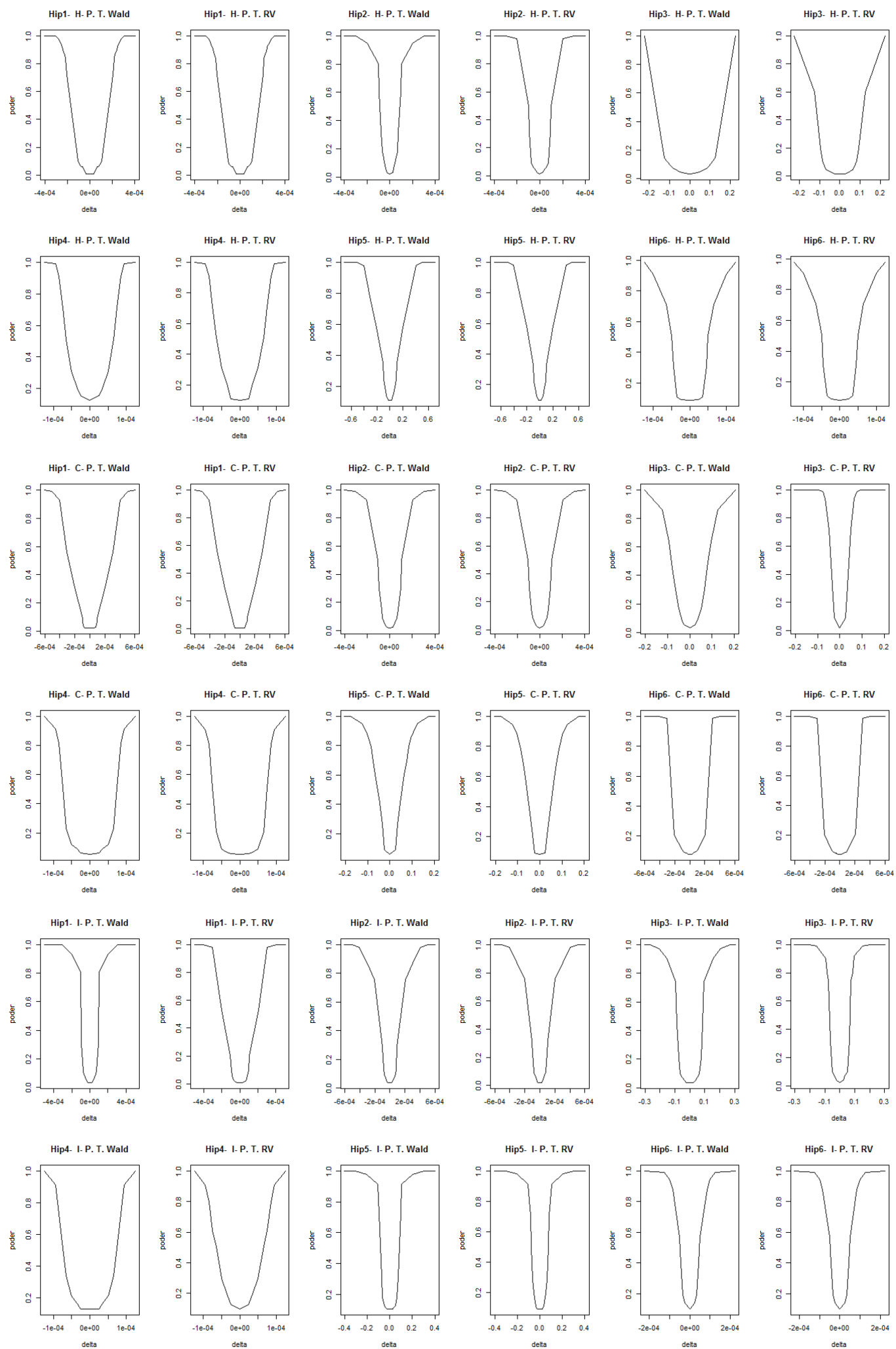

Figura 42: Gráficos do P. T. de Wald e RV para 3 réplicas- distribuição t de Student3 laboratórios e 5 rotações. 

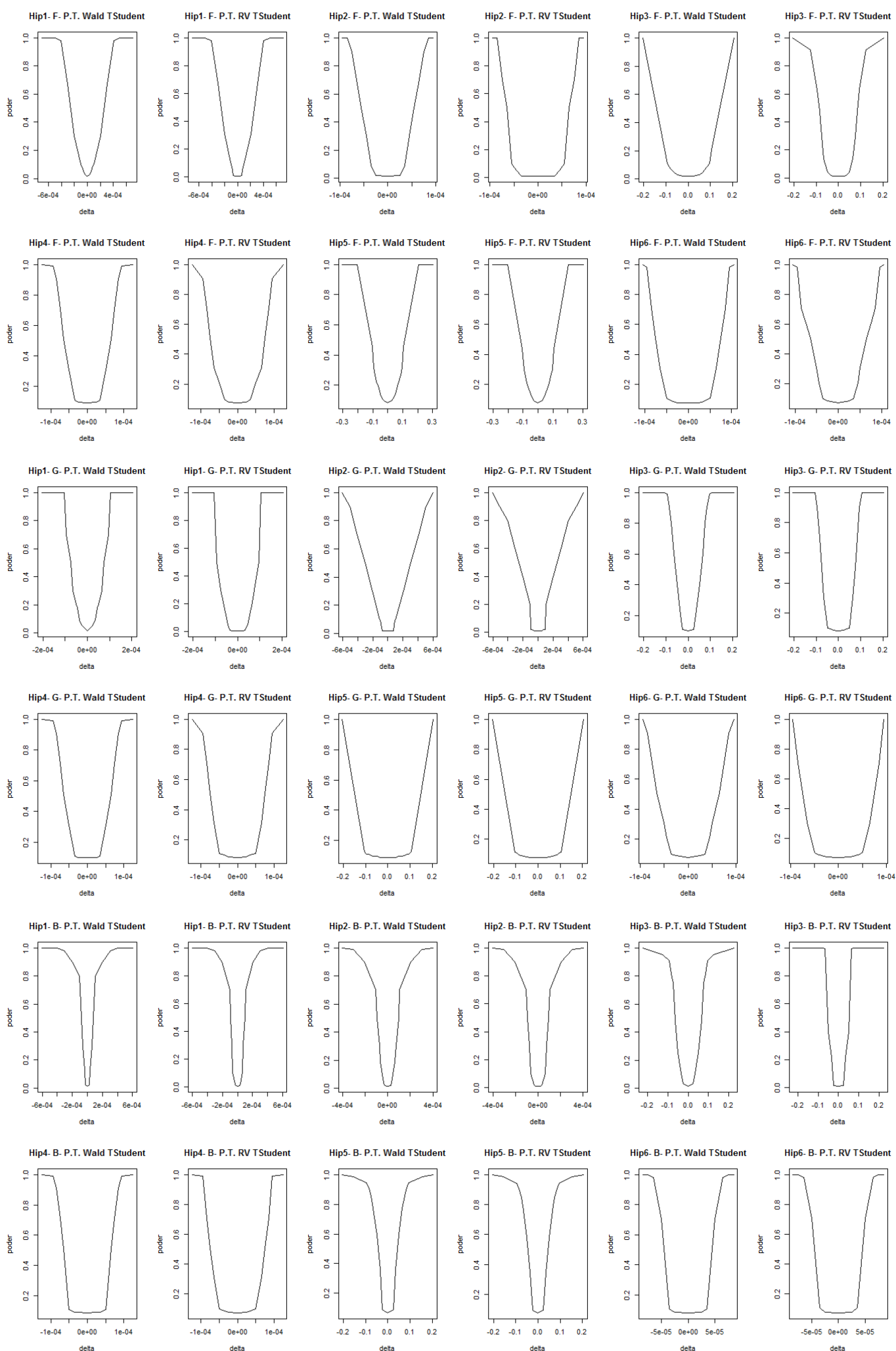

Figura 43: Gráficos do P. T. de Wald e RV para 5 réplicas- distribuição t de Student3 laboratórios e 5 rotações. 

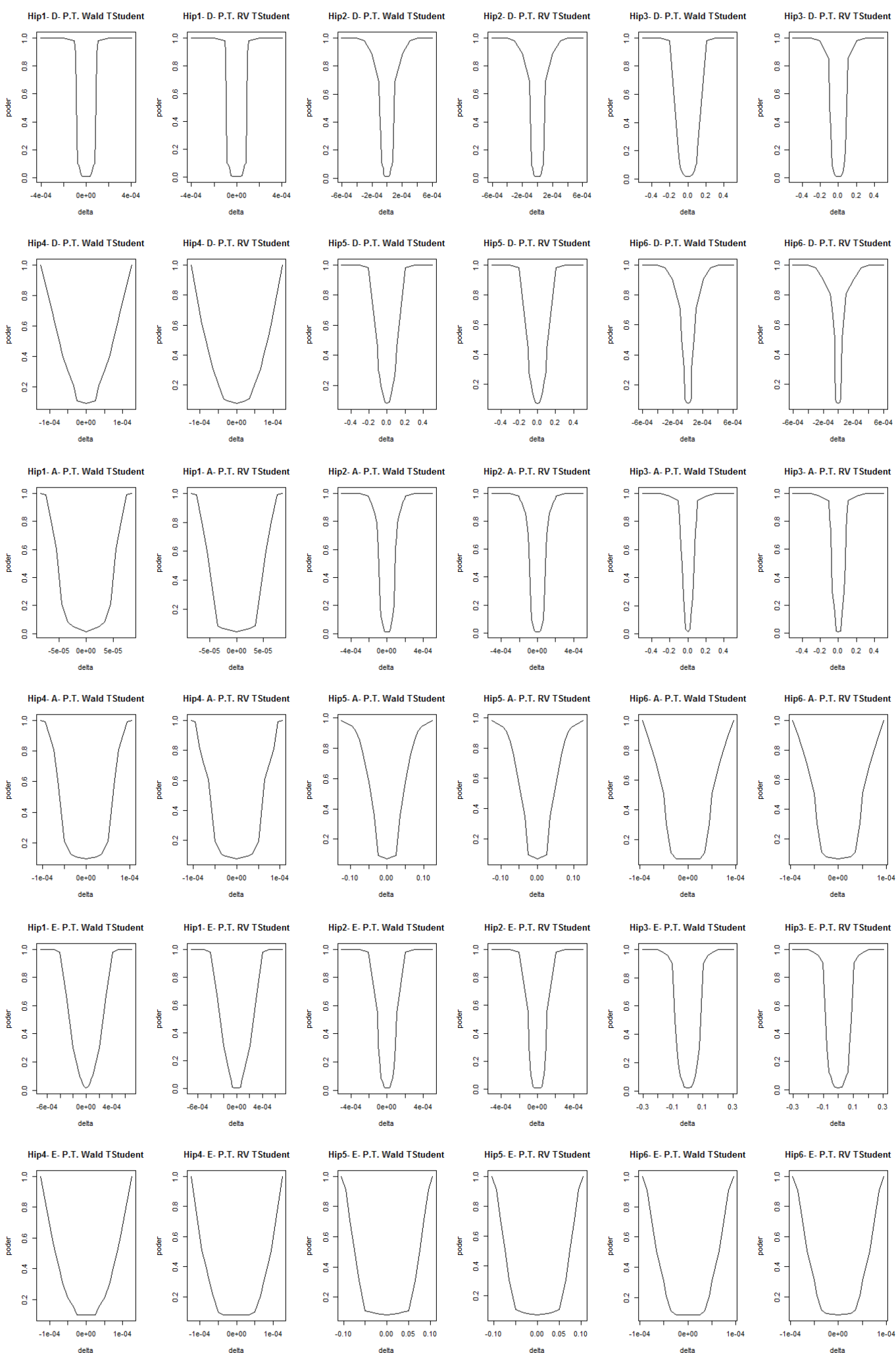

Figura 44: Gráficos do P. T. de Wald e RV para 5 réplicas- distribuição t de Student3 laboratórios e 5 rotações. 

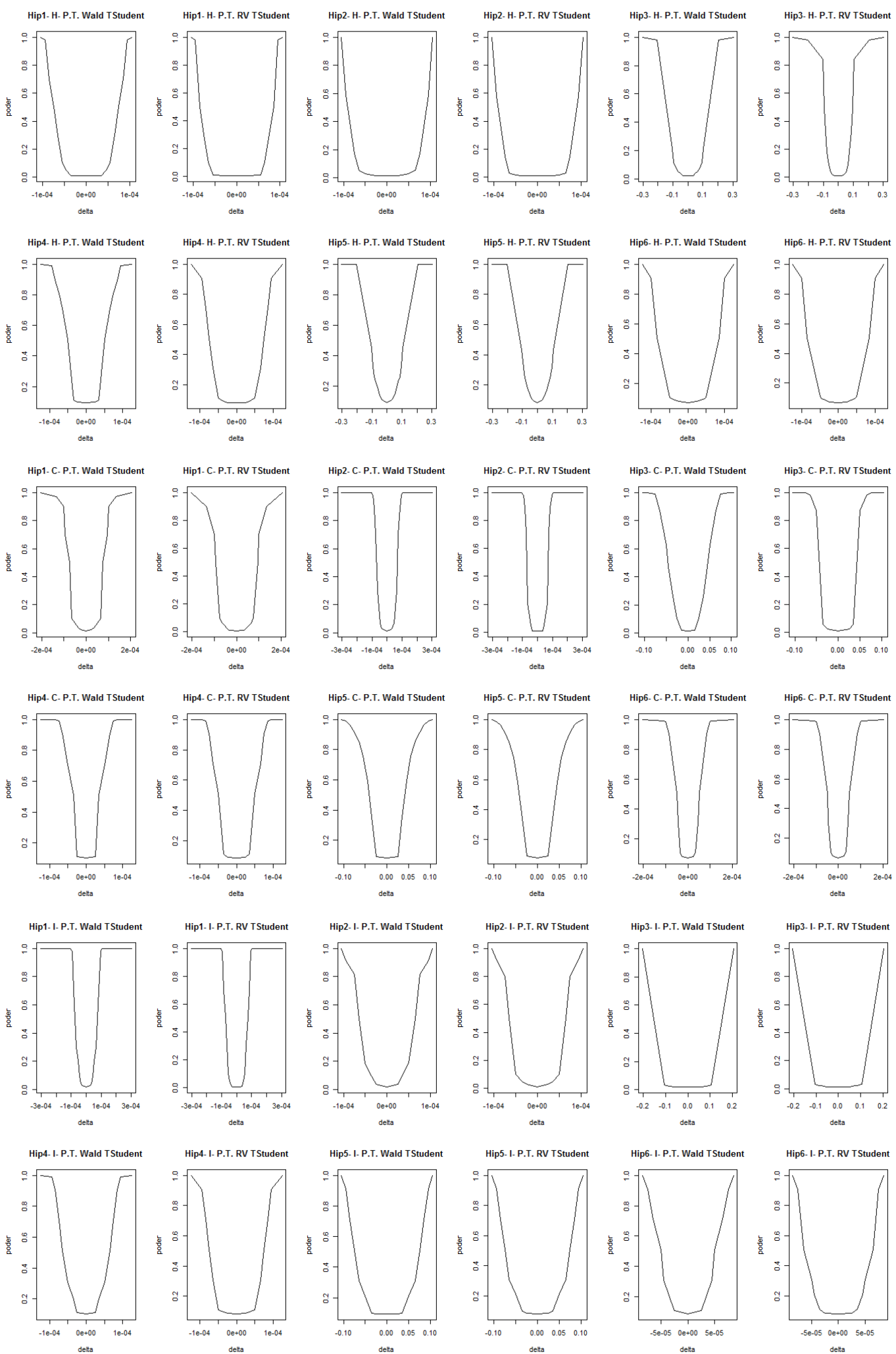

Figura 45: Gráficos do P. T. de Wald e RV para 5 réplicas- distribuição t de Student3 laboratórios e 5 rotações. 

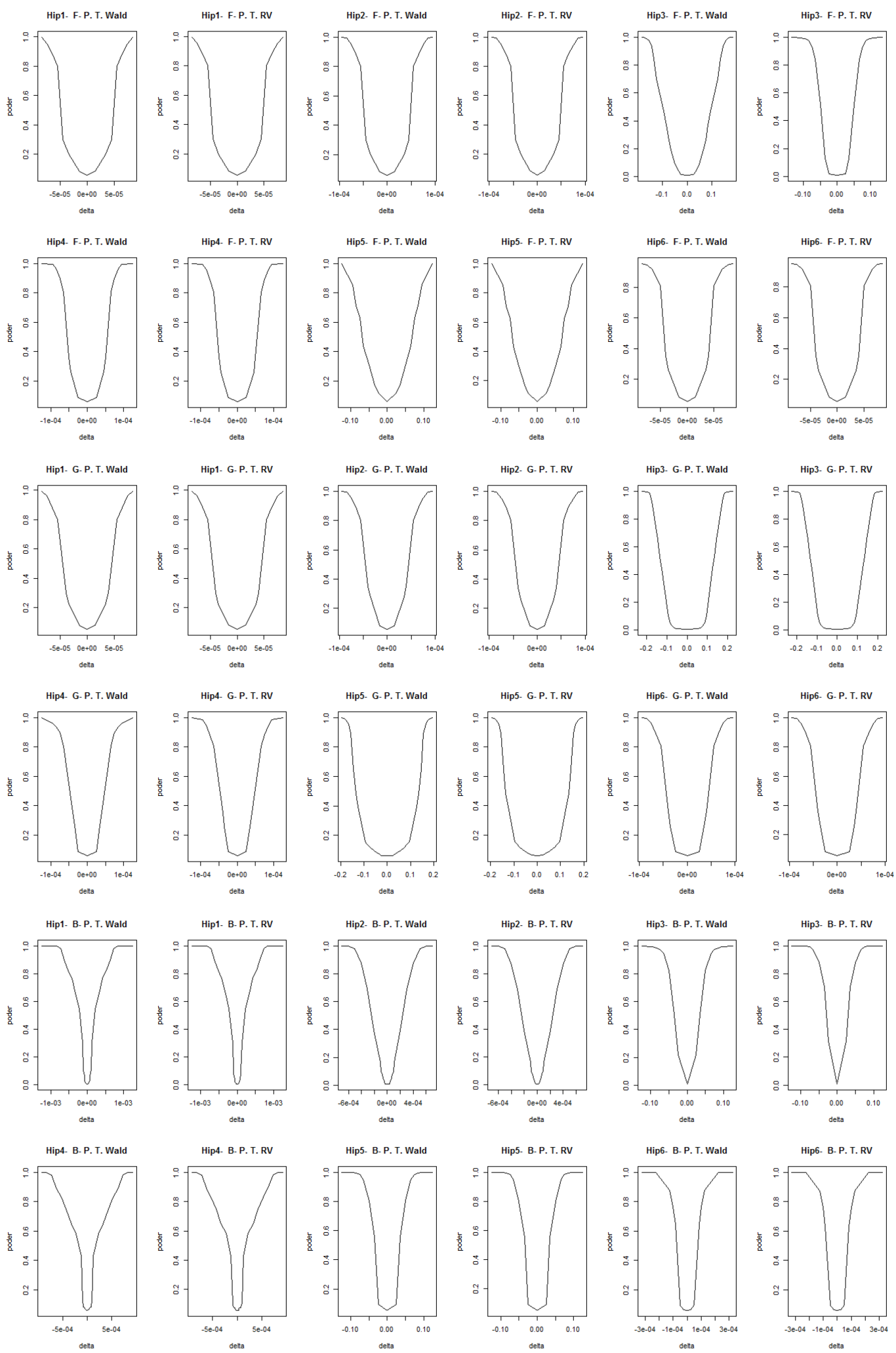

Figura 46: Gráficos do P. T. de Wald e RV para 20 réplicas- distribuição t de Student3 laboratórios e 5 rotações. 

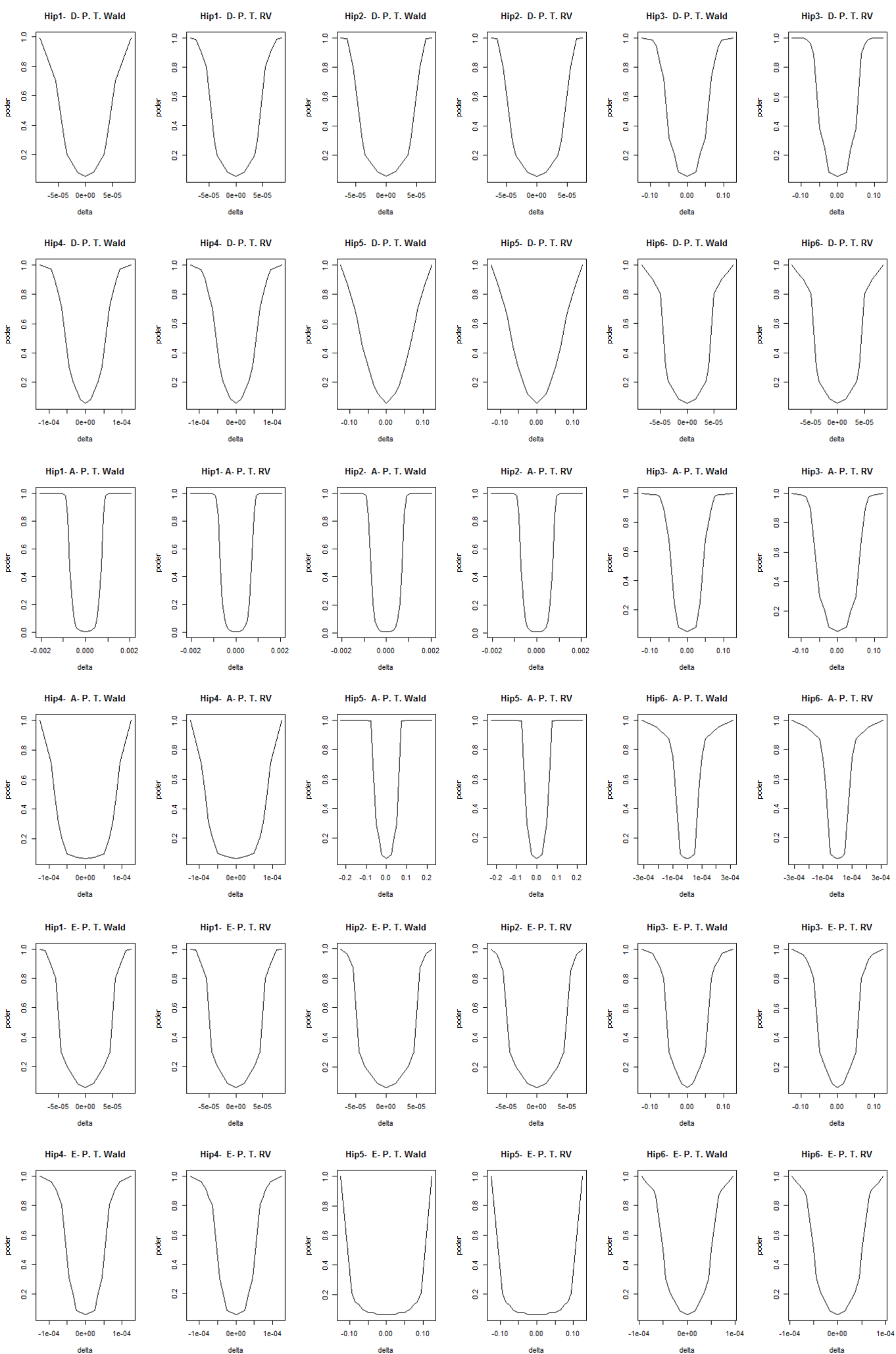

Figura 47: Gráficos do P. T. de Wald e RV para 20 réplicas- distribuição t de Student3 laboratórios e 5 rotações. 

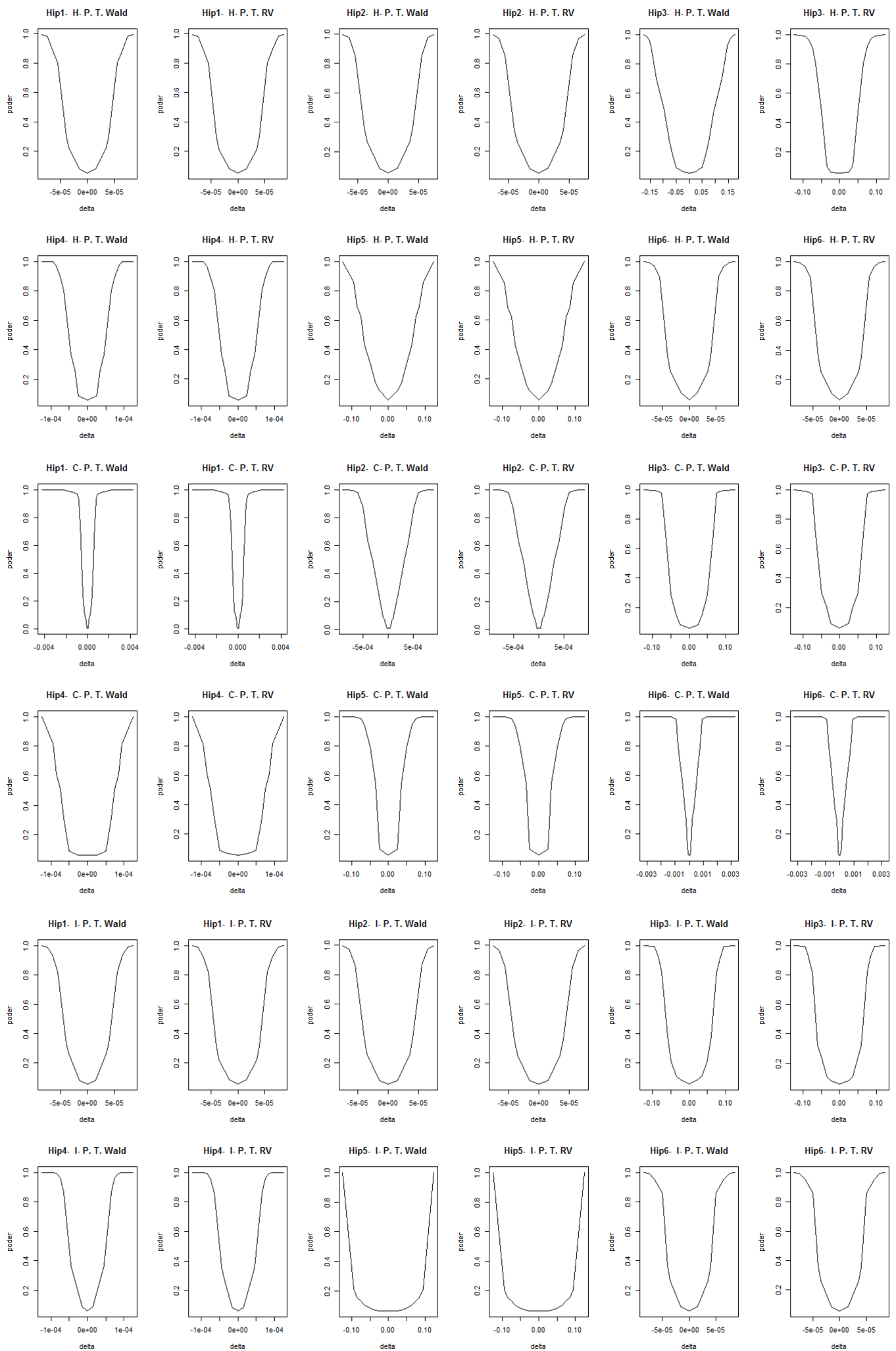

Figura 48: Gráficos do P. T. de Wald e RV para 20 réplicas- distribuição t de Student3 laboratórios e 5 rotações. 


\section{Apêndice D}

Tabela 37: Conjunto de dados usado na aplicação (dispostos na ordem Código (C),Faixa (F) e Potência Corrigida (PC) ).

\begin{tabular}{|c|c|c|c|c|c|c|c|c|}
\hline C & $\mathbf{F}$ & PC & $\mathrm{C}$ & $\mathrm{F}$ & $\mathrm{PC}$ & $\mathrm{C}$ & $\mathrm{F}$ & $\mathrm{PC}$ \\
\hline 1 & 1500 & 19,76 & 1 & 5500 & 80,35 & 2 & 4500 & 68,75 \\
\hline 1 & 1500 & 19,96 & 1 & 5500 & 80,33 & 2 & 4500 & 69,48 \\
\hline 1 & 1500 & 19,78 & 1 & 5500 & 80,21 & 2 & 4500 & 71,67 \\
\hline 1 & 1500 & 19,85 & 1 & 5500 & 80,14 & 2 & 5000 & 76,95 \\
\hline 1 & 1500 & 19,64 & 1 & 6000 & 80,13 & 2 & 5000 & 76,66 \\
\hline 1 & 2000 & 27,23 & 1 & 6000 & 80,35 & 2 & 5000 & 74,85 \\
\hline 1 & 2000 & 27,33 & 1 & 6000 & 80,23 & 2 & 5000 & 75,42 \\
\hline 1 & 2000 & 27,24 & 1 & 6000 & 79,13 & 2 & 5000 & 77,25 \\
\hline 1 & 2000 & 27,17 & 1 & 6000 & 79,94 & 2 & 5500 & 79,86 \\
\hline 1 & 2000 & 27,30 & 2 & 1500 & 19,77 & 2 & 5500 & 80,37 \\
\hline 1 & 2500 & 35,57 & 2 & 1500 & 19,7 & 2 & 5500 & 78,22 \\
\hline 1 & 2500 & 35,51 & 2 & 1500 & 19,51 & 2 & 5500 & 78,71 \\
\hline 1 & 2500 & 35,45 & 2 & 1500 & 19,62 & 2 & 5500 & 80,84 \\
\hline 1 & 2500 & 35,39 & 2 & 1500 & 20,02 & 2 & 6000 & 79,66 \\
\hline 1 & 2500 & 35,55 & 2 & 2000 & 27,41 & 2 & 6000 & 79,85 \\
\hline 1 & 3000 & 45,61 & 2 & 2000 & 27,43 & 2 & 6000 & 77,55 \\
\hline 1 & 3000 & 45,60 & 2 & 2000 & 26,97 & 2 & 6000 & 77,69 \\
\hline 1 & 3000 & 45,53 & 2 & 2000 & 27,27 & 2 & 6000 & 81,46 \\
\hline 1 & 3000 & 45,54 & 2 & 2000 & 27,62 & 3 & 1500 & 19,06 \\
\hline 1 & 3000 & 45,34 & 2 & 2500 & 35,47 & 3 & 1500 & 19,14 \\
\hline 1 & 3500 & 54,52 & 2 & 2500 & 35,54 & 3 & 1500 & 19,17 \\
\hline 1 & 3500 & 54,25 & 2 & 2500 & 35,35 & 3 & 1500 & 19,23 \\
\hline 1 & 3500 & 54,42 & 2 & 2500 & 35,61 & 3 & 1500 & 19,18 \\
\hline 1 & 3500 & 54,32 & 2 & 2500 & 36,08 & 3 & 2000 & 26,43 \\
\hline 1 & 3500 & 54,32 & 2 & 3000 & 45,46 & 3 & 2000 & 26,37 \\
\hline 1 & 4000 & 63,68 & 2 & 3000 & 45,4 & 3 & 2000 & 26,41 \\
\hline 1 & 4000 & 63,17 & 2 & 3000 & 45,16 & 3 & 2000 & 26,45 \\
\hline 1 & 4000 & 63,13 & 2 & 3000 & 45,73 & 3 & 2000 & 26,37 \\
\hline 1 & 4000 & 63,08 & 2 & 3000 & 46,05 & 3 & 2500 & 34,37 \\
\hline 1 & 4000 & 63,39 & 2 & 3500 & 54,25 & 3 & 2500 & 34,25 \\
\hline 1 & 4500 & 71,48 & 2 & 3500 & 54,51 & 3 & 2500 & 34,24 \\
\hline 1 & 4500 & 71,56 & 2 & 3500 & 53,89 & 3 & 2500 & 34,21 \\
\hline 1 & 4500 & 71,50 & 2 & 3500 & 54,22 & 3 & 2500 & 34,21 \\
\hline 1 & 4500 & 71,53 & 2 & 3500 & 55,31 & 3 & 3000 & 43,84 \\
\hline 1 & 4500 & 70,91 & 2 & 4000 & 63,73 & 3 & 3000 & 43,48 \\
\hline 1 & 5000 & 76,99 & 2 & 4000 & 63,54 & 3 & 3000 & 43,57 \\
\hline 1 & 5000 & 77,01 & 2 & 4000 & 62,3 & 3 & 3000 & 43,43 \\
\hline 1 & 5000 & 76,78 & 2 & 4000 & 62,49 & 3 & 3000 & 43,52 \\
\hline 1 & 5000 & 76,992 & 2 & 4000 & 63,89 & 3 & 3500 & 52,54 \\
\hline 1 & 5000 & 76,85 & 2 & 4500 & 71,01 & 3 & 3500 & 52,22 \\
\hline 1 & 5500 & 80,06 & 2 & 45096 & 71,25 & 3 & 3500 & 52,19 \\
\hline
\end{tabular}


Tabela 38: Conjunto de dados usado na aplicação (dispostos na ordem Código (C),Faixa (F) e Potência Corrigida (PC) ).

\begin{tabular}{|c|c|c|c|c|c|c|c|c|}
\hline $\mathrm{C}$ & $\bar{F}$ & $\mathrm{PC}$ & $\mathrm{C}$ & $\mathrm{F}$ & $\mathrm{PC}$ & $\mathrm{C}$ & $\mathrm{F}$ & $\mathrm{PC}$ \\
\hline 3 & 3500 & 51,95 & 4 & 2500 & 35,7 & 5 & 2000 & 27,06 \\
\hline 3 & 3500 & 51,92 & 4 & 3000 & 45,5 & 5 & 2000 & 27,33 \\
\hline 3 & 4000 & 61,16 & 4 & 3000 & 45,5 & 5 & 2000 & 27,01 \\
\hline 3 & 4000 & 61,1 & 4 & 3000 & 45,5 & 5 & 2000 & 26,97 \\
\hline 3 & 4000 & 61,34 & 4 & 3000 & 45,6 & כ & 2000 & 27,13 \\
\hline 3 & 4000 & 61,14 & 4 & 3000 & 45,7 & 5 & 2500 & 35,56 \\
\hline 3 & 4000 & 61,07 & 4 & 3500 & 54,3 & 5 & 2500 & 35,43 \\
\hline 3 & 4500 & 68,78 & 4 & 3500 & 54,3 & 5 & 2500 & 35,15 \\
\hline 3 & 4500 & 68,48 & 4 & 3500 & 54,4 & 5 & 2500 & 35,05 \\
\hline 3 & 4500 & 68,83 & 4 & 3500 & 54,4 & 5 & 2500 & 35,26 \\
\hline 3 & 4500 & 68,66 & 4 & 3500 & 54,4 & 5 & 3000 & 45,47 \\
\hline 3 & 4500 & 68,48 & 4 & 4000 & 63,4 & 5 & 3000 & 45,4 \\
\hline 3 & 5000 & 74,57 & 4 & 4000 & 63,6 & 5 & 3000 & 45,18 \\
\hline 3 & 5000 & 73,99 & 4 & 4000 & 63,5 & 5 & 3000 & 44,91 \\
\hline 3 & 5000 & 74,02 & 4 & 4000 & 63,6 & & 3000 & 45 \\
\hline 3 & 5000 & 74,12 & 4 & 4000 & 63,8 & 5 & 3500 & 54,42 \\
\hline 3 & 5000 & 73,94 & 4 & 4500 & 71,2 & 5 & 3500 & 54,35 \\
\hline 3 & 5500 & 77,83 & 4 & 4500 & 71,4 & 5 & 3500 & 54,05 \\
\hline 3 & 5500 & 77,53 & 4 & 4500 & 71,3 & 5 & 3500 & 53,9 \\
\hline 3 & 5500 & 77,73 & 4 & 4500 & 71,2 & 5 & 3500 & 53,92 \\
\hline 3 & 5500 & 77,77 & 4 & 4500 & 71,5 & 5 & 4000 & 63,2 \\
\hline 3 & 5500 & 77,59 & 4 & 5000 & 76,3 & 5 & 4000 & 62,53 \\
\hline 3 & 6000 & 78,45 & 4 & 5000 & 76,5 & 5 & 4000 & 62,58 \\
\hline 3 & 6000 & 78,41 & 4 & 5000 & 76,4 & 5 & 4000 & 62,7 \\
\hline 3 & 6000 & 78,55 & 4 & 5000 & 76,3 & 5 & 4000 & 62,67 \\
\hline 3 & 6000 & 78,67 & 4 & 5000 & 76,6 & 5 & 4500 & 71,04 \\
\hline 3 & 6000 & 78,67 & 4 & 5500 & 78,6 & 5 & 4500 & 70,6 \\
\hline 4 & 1500 & 19,8 & 4 & 5500 & 78,8 & 5 & 4500 & 70,46 \\
\hline 4 & 1500 & 19,7 & 4 & 5500 & 78,7 & 5 & 4500 & 70,39 \\
\hline 4 & 1500 & 19,7 & 4 & 5500 & 78,5 & 5 & 4500 & 70,36 \\
\hline 4 & 1500 & 19,8 & 4 & 5500 & 78,9 & 5 & 5000 & 76,43 \\
\hline 4 & 1500 & 20 & 4 & 6000 & 78 & 5 & 5000 & 76,14 \\
\hline 4 & 2000 & 27,4 & 4 & 6000 & 78 & 5 & 5000 & 76 \\
\hline 4 & 2000 & 27,4 & 4 & 6000 & 78 & 5 & 5000 & 75,95 \\
\hline 4 & 2000 & 27,4 & 4 & 6000 & 78 & 5 & 5000 & 75,9 \\
\hline 4 & 2000 & 27,4 & 4 & 6000 & 78,2 & 5 & 5500 & 79,51 \\
\hline 4 & 2000 & 27,5 & 5 & 1500 & 19,7 & 5 & 5500 & 78,89 \\
\hline 4 & 2500 & 35 & 5 & 1500 & 9,67 & 5 & 5500 & 79,28 \\
\hline 4 & 2500 & 35,5 & 5 & 1500 & 19,56 & 5 & 5500 & 79,26 \\
\hline 4 & 2500 & 35,5 & 5 & 1500 & 19,38 & 5 & 5500 & 79,22 \\
\hline 4 & 2500 & 35,6 & 5 & 1500 & 19,56 & 5 & 6000 & 78,85 \\
\hline
\end{tabular}


Tabela 39: Conjunto de dados usado na aplicação (dispostos na ordem Código (C), Faixa (F) e Potência Corrigida (PC) ).

\begin{tabular}{|c|c|c|c|c|c|}
\hline $\mathrm{C}$ & $\mathrm{F}$ & $\mathrm{PC}$ & $\mathrm{C}$ & $\mathrm{F}$ & $\mathrm{PC}$ \\
\hline 5 & 6000 & 78,59 & 6 & 5000 & 74,8 \\
\hline 5 & 6000 & 78,79 & 6 & 5000 & 74,8 \\
\hline 5 & 6000 & 78,88 & 6 & 5000 & 74,8 \\
\hline 5 & 6000 & 78,76 & 6 & 5500 & 78,6 \\
\hline 6 & 1500 & 19,6 & 6 & 5500 & 78,3 \\
\hline 6 & 1500 & 19,5 & 6 & 5500 & 78,4 \\
\hline 6 & 1500 & 19,6 & 6 & 5500 & 78,4 \\
\hline 6 & 1500 & 19,5 & 6 & 5500 & 78,5 \\
\hline 6 & 1500 & 19,6 & 6 & 6000 & 79,1 \\
\hline 6 & 2000 & 27 & 6 & 6000 & 79 \\
\hline 6 & 2000 & 26,8 & 6 & 6000 & 78,9 \\
\hline 6 & 2000 & 27 & 6 & 6000 & 79,1 \\
\hline 6 & 2000 & 26,8 & 6 & 6000 & 79,1 \\
\hline 6 & 2000 & 26,9 & & & \\
\hline 6 & 2500 & 35 & & & \\
\hline 6 & 2500 & 34,9 & & & \\
\hline 6 & 2500 & 35 & & & \\
\hline 6 & 2500 & 34,9 & & & \\
\hline 6 & 2500 & 34,9 & & & \\
\hline 6 & 3000 & 45,2 & & & \\
\hline 6 & 3000 & 45 & & & \\
\hline 6 & 3000 & 45,1 & & & \\
\hline 6 & 3000 & 45 & & & \\
\hline 6 & 3000 & 45,1 & & & \\
\hline 6 & 3500 & 53,8 & & & \\
\hline 6 & 3500 & 53,6 & & & \\
\hline 6 & 3500 & 53,6 & & & \\
\hline 6 & 3500 & 53,6 & & & \\
\hline 6 & 3500 & 53,7 & & & \\
\hline 6 & 4000 & 62,3 & & & \\
\hline 6 & 4000 & 62,1 & & & \\
\hline 6 & 4000 & 62,2 & & & \\
\hline 6 & 4000 & 62,1 & & & \\
\hline 6 & 4000 & 62,2 & & & \\
\hline 6 & 4500 & 69,8 & & & \\
\hline 6 & 4500 & 69,7 & & & \\
\hline 6 & 4500 & 69,7 & & & \\
\hline 6 & 4500 & 69,7 & & & \\
\hline 6 & 4500 & 69,7 & & & \\
\hline 6 & 5000 & 75 & & & \\
\hline 6 & 5000 & 74,8 & & & \\
\hline
\end{tabular}

